\title{
Link between Muscle Function and Physical Function in Critically III Patients
}

by

Shanita Chhiba

Thesis presented in fulfilment of the requirements for the degree of Master of Science in Physiotherapy at the Faculty of Health Sciences at Stellenbosch University

Primary Supervisor: Professor SD Hanekom, Department of Interdisciplinary Health Sciences, Stellenbosch University

Secondary Supervisor: Dr A Lupton- Smith, Department of Interdisciplinary Health Sciences, Stellenbosch University 


\section{DECLARATION}

By submitting this thesis electronically, I declare that the entirety of the work contained therein is my own, original work, that I am the sole author thereof (save to the extent explicitly otherwise stated), that reproduction and publication thereof by Stellenbosch University will not infringe any third-party rights and that I have not previously in its entirety or in part submitted it for obtaining any qualification.

Shanita Chhiba

March 2021

Copyright @ 2021 Stellenbosch University

All rights reserved 


\section{ABSTRACT}

Introduction: Improvements in intensive care unit (ICU) care has led to a growing number of survivors of critical illness, but not necessarily improved quality of life. After surviving critical illness, many critically ill survivors suffer from long-term complications such as reduced physical function, mental and cognitive dysfunction and a reduced health-related quality of life (HRQoL). Critically ill survivors develop a loss of muscle mass and muscle weakness of the respiratory and peripheral muscles. Therefore, the premise of this thesis was to determine whether muscle structure and function is associated with physical function in critically ill survivors.

Methods: A scoping review was done to map the current understanding of muscle structure and function in critically ill patients. Six databases were searched using a data-base specific search strategy and papers were identified based on a familiarity with the literature. The primary investigator $(\mathrm{PI})$ used a systematic process to extract data into a self-developed excel spreadsheet. The data obtained were used to inform the planning of the primary research study. A prospective observational cohort study was conducted to describe the outcomes of critically ill patients as well as determine the correlation between muscle structure, muscle function and physical function in critically ill patients at ICU discharge and hospital discharge. Mechanically ventilated participants were recruited within 24 hours of admission to the ICU. Measurements taken of muscle structure and function included ultrasonography of the diaphragm and peripheral muscles, the Medical Research CouncilSum Score (MRC-SS), dynamometry, maximal inspiratory pressure (MIP) and respiratory muscle endurance. The physical function and HRQoL measurements included the Chelsea Critical Care Physical Assessment (CPAx) tool, the de Morton Mobility Index (DEMMI), the Six-Minute Walk Test (6-MWT) and the EQ-5D-5L questionnaire. Associations between measures of muscle structure, muscle function and physical function were reported using Spearman's correlations. 
Results: One thousand two hundred and eighty-two studies were retrieved and assessed for inclusion into the scoping review. After assessing for eligibility, thirty-four studies were included in the review. Data regarding muscle structure and function were predominantly from the developed countries. Assessments were focused on either muscle structure or muscle function, and not both. The methodology for the assessments of muscle structure and function were not standardised. Majority of assessments took place during ICU stay. The postural muscles were the least investigated. Forty-five participants were recruited for the primary research study. The median age of the sample was $34.5(24.3-47.4)$ years. Participants presented with changes in respiratory and peripheral muscle structure and function, and in physical function. A strong positive correlation was established between MIP and the 6-MWT (rho $=0.75, \mathrm{p}<0.01)$.

Conclusion: The methodologies used to measure muscle structure and function were not standardised and the discrepancy in results hampered our current understanding of muscle structure and function in critically ill patients. Muscle structure and function may partially explain physical function outcomes in survivors of critical illness, but other variables need to be considered.

Word count: 485 


\section{UITTREKSEL}

Inleiding: Verbeterings in intensiewesorgeenheid (ISE) sorg het gelei tot 'n groeiende aantal oorlewendes van kritieke siektes, maar nie noodwendig verbeterde lewensgehalte nie. $\mathrm{Na}$ die oorlewing van kritieke siekte, baie krities siek oorlewendes ly aan langtermyn komplikasies, soos verminderde fisiese funksie, geestelike en kognitiewe disfunksie, en 'n verminderde gesondheidsverwante lewensgehalte (HRQoL). Krities siek oorlewendes ontwikkel 'n verlies van spiermassa en spierswakheid van die respiratoriese en perifere spiere. Daarom was die uitgangspunt van hierdie proefskrif om te bepaal of spierstruktuur en funksie geassosieer word met fisiese funksie in kritiek- siek oorlewendes.

Metodes: 'n literatuuroorsig is uitgevoer om die huidige begrip van spierstruktuur en -funksie by kritiek siek pasiënte te bepaal. Ses databasisse is deursoek met behulp van 'n spesifieke soekstrategie. Artikels is geïdentifiseer van 'n vertroudheid met die literatuur. Die primêre ondersoeker (PI) het 'n sistematiese proses gebruik om data in 'n selfontwikkelde Excelsigblad te onttrek. Die data wat verkry is, is gebruik om die beplanning van die primêre navorsingstudie te rig. 'n Voornemende waarnemings kohort studie is uitgevoer om die uitkomste van krities siek pasiënte te beskryf, asook om die korrelasie tussen spierfunksie en fisiese funksie in krities siek pasiënte tydens ISE-ontslag en hospitaal-ontslag te bepaal. Meganiese geventileerde deelnemers is binne 24 uur na toelating tot die ISE gewerf. Metings van spierstruktuur en funksie sluit in ultrasonografie van die diafragma en perifere spiere, mediese navorsing raad-som telling (MRC-SS), dinamometrie, maksimum inspirerende druk (MIP) en respiratoriese spiere uithouvermoë. Die fisiese funksie en HRQoL-metings het ingesluit die Chelsea Kritieke Sorg Fisiese Assesserings (CPAx) instrument, die de Morton Mobiliteits indeks (DEMMI), die Ses-Minute Staptoets (6-MWT) en die EQ-5D-5L-vraelys. Verbindings tussen maatstawwe van spierstruktuur, spierfunksie en fisiese funksie is aangemeld met behulp van Spearman se korrelasies. 
Resultate: Een duisend twee honderd twee-en-tagtig studies is opgespoor en geassesseer vir insluiting in die literatuuroorsig. Na die sifting is vier-en-dertig studies ingesluit. Data met betrekkiing tot spierstruktuur en -funksie was hoofsaaklik uit die ontwikkelde lande. Assesserings was gefokus op spierstruktuur of spierfunksie, en nie albei nie. Die metodologie vir die assesserings van spierstruktuur en -funksie is nie gestandaardiseer nie. Meerderheid van die assesserings het plaasgevind tydens ISE verblyf. Die posturale spiere was die minste ondersoek. Vyf en veertig deelnemers is vir die primêre navorsingstudie gewerf. Die mediaanouderdom van die steekproef was 34.5 (24.3 - 47.4) jaar. Deelnemers het aangebied met gestremdhede in spierstruktuur, spierkrag en fisiese funksie.'n Sterk positiewe korrelasie is vasgestel tussen MIP en die 6 MWT (rho $=0.75, p<0.01$ ).

Gevolgtrekking: Die metodologieë wat gebruik word om spierstruktuur en -funksie te meet is nie gestandaardiseer nie en die verskil in resultate belemmer ons huidige begrip van spierstruktuur en -funksie by kritiek siek pasiënte. Spierstruktuur en -funksie kan die fisieke funksie-uitkomste gedeeltelik by oorlewendes van kritieke siekte verklaar, maar ander veranderlikes moet in ag geneem word.

Woorde: 460 


\section{ACKNOWLEDGMENTS}

\section{Supervisors}

Professor SD Hanekom and Dr A Lupton-Smith from the Department of Interdisciplinary Health Sciences of Stellenbosch University for all their guidance, helpful suggestions, time and support.

\section{ICU Physicians}

Dr N Ahmed and Dr U Lalla for the willingness and help during the study.

\section{ICU Nursing Staff}

For their support and help during the research study.

\section{Participants}

For their cooperation and willingness to participate.

\section{Family - Mum, Ajima, Hasita and Rakesh}

For their support, understanding, love and words of encouragement.

\section{Dad}

For your guidance and for always watching over me.

\section{Friends}

For their support and understanding. 


\section{Table of Contents}

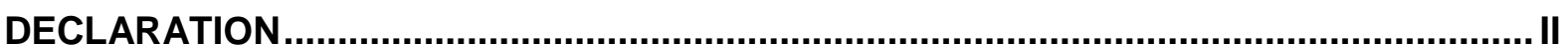

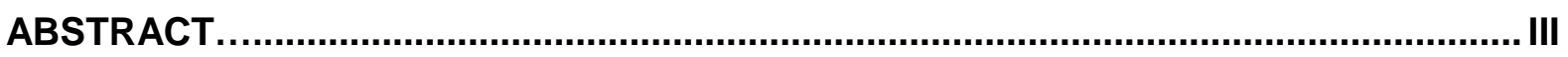

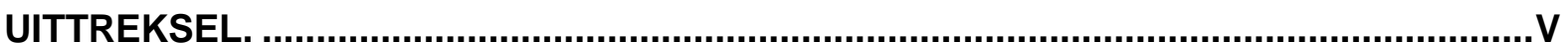

ACKNOWLEDGMENTS .....................................................................................

LIST OF TABLES

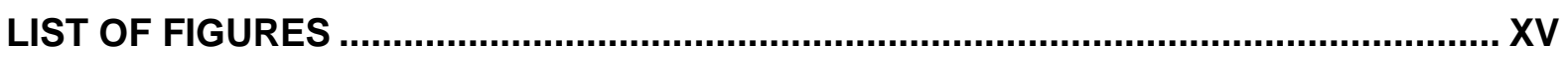

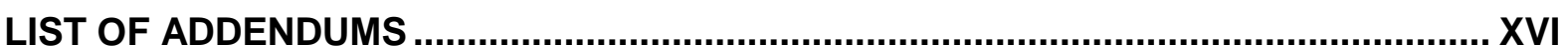

ABBREVIATIONS

GLOSSARY

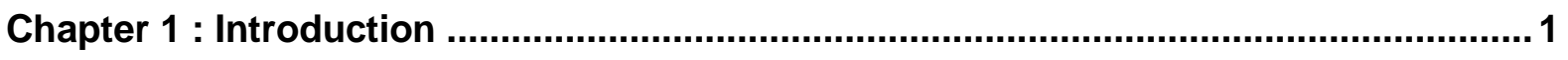

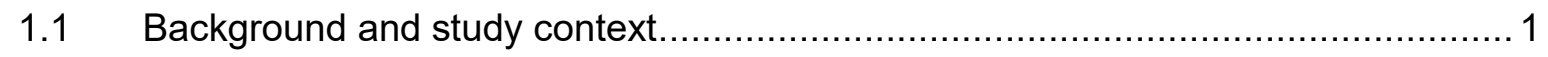

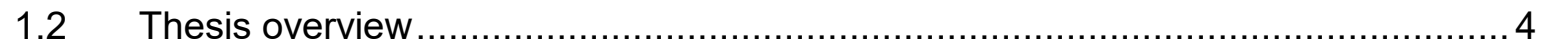

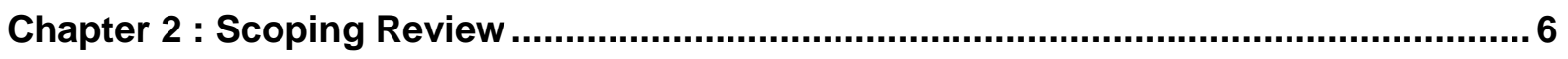

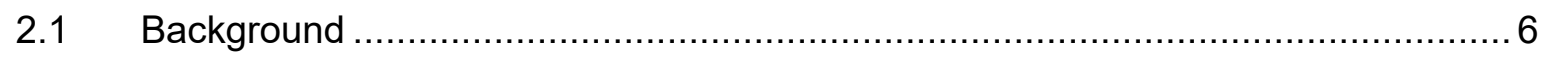

2.2 Methods

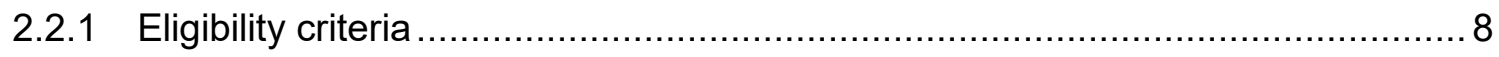

2.2.2 Information sources and search strategy ………....................................... 8

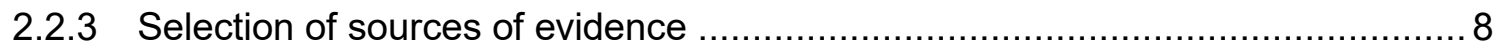

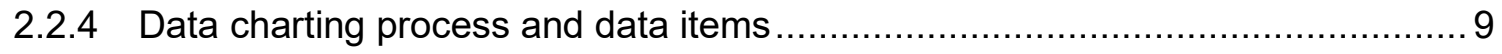

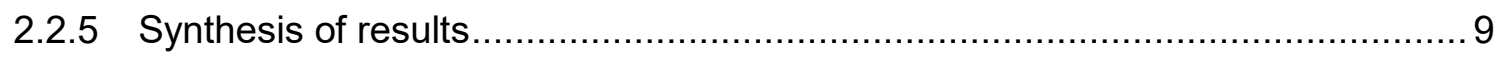

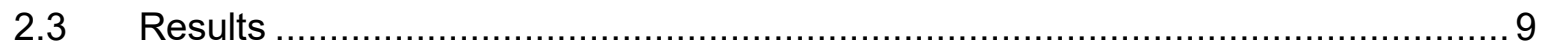

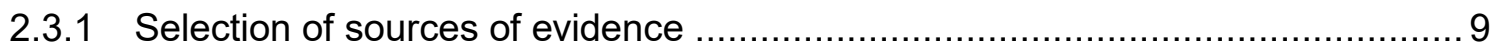


2.3.2 Authors, year of publication, country of origin, sample size and sample population

2.3.3 Type of skeletal muscle and the different type of measurement tools 14

2.3.4 Frequency of assessments 17

2.3.5 Testing procedure of the measurement tools for muscle structure and function20

2.3.6 Changes in muscle structure and function reported in critically ill patients 22

2.3.7 Factors associated with change in muscle structure and function 28

2.4 Discussion 31

2.5 Limitation and recommendation 33

2.6 Conclusion 34

Chapter 3 : Methodology of the Primary Research Study. 35

3.1 Research question 35

3.2 Research aim 35

3.3 Research objectives 35

3.3.1 Respiratory muscle structure and function in a critically ill population ...... 35

3.3.2 Peripheral muscle structure and function in a critically ill population 35

3.3.3 Physical function and health-related quality of life (HRQoL) in a critically ill population 36

3.3.4 Associations between muscle structure, muscle function and physical function in a critically ill population 36

3.3.5 Demographics and clinical characteristics. 37

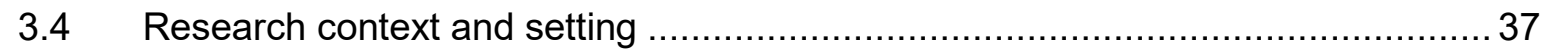

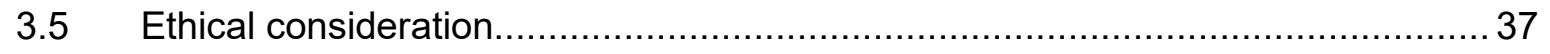

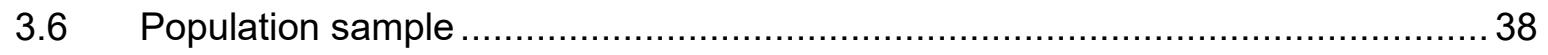

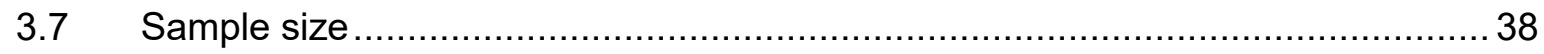




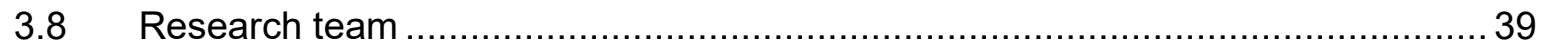

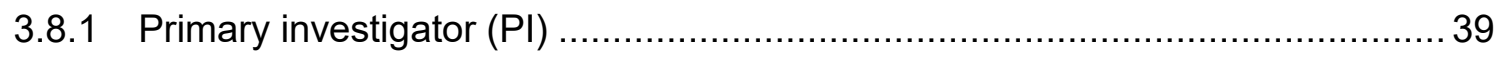

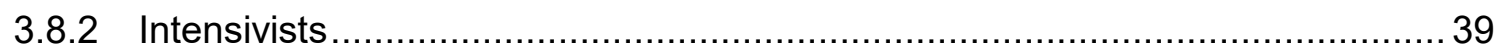

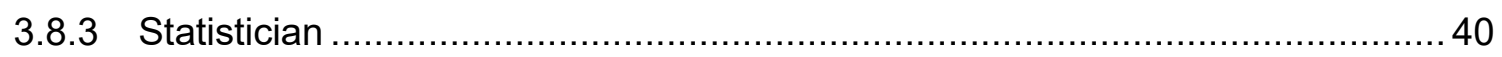

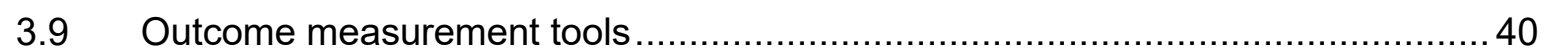

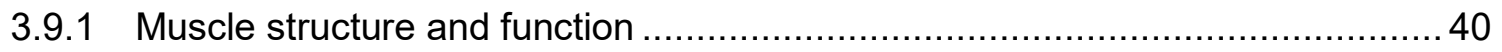

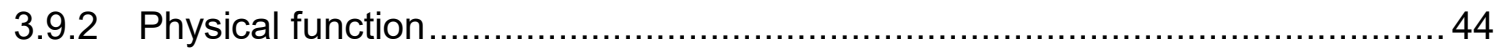

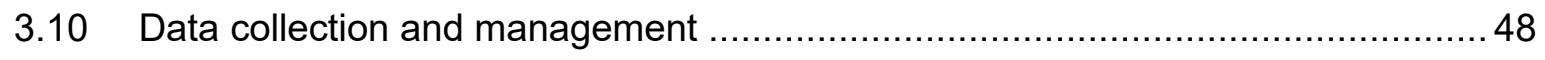

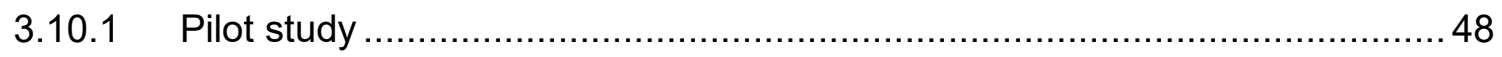

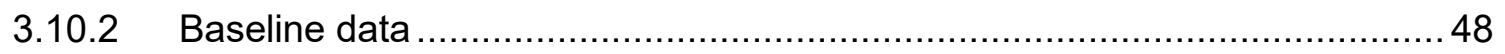

3.10.3 Measures of muscle structure, muscle function, physical function and health-

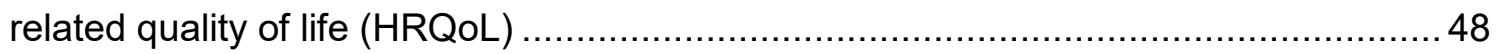

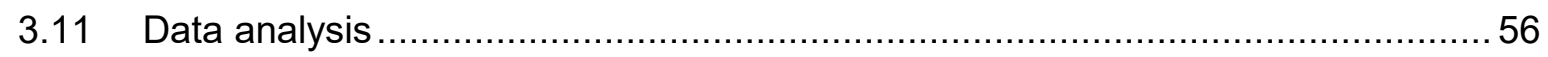

Chapter 4 : Results of the Primary Research Study ..................................................57

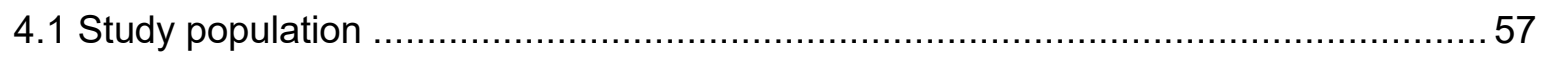

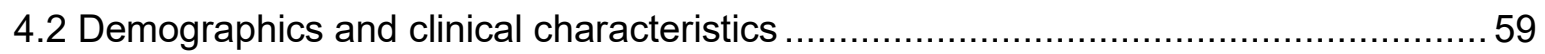

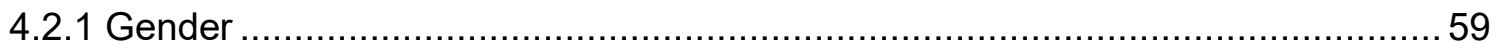

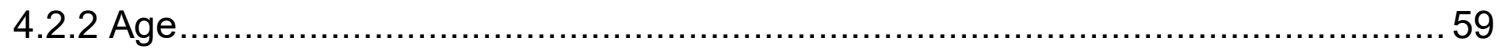

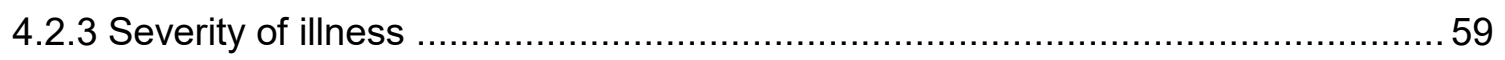

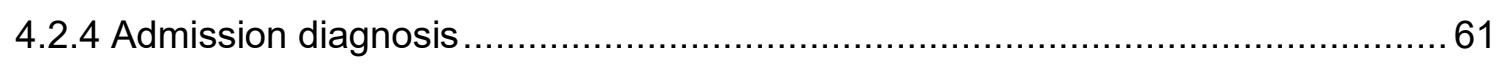

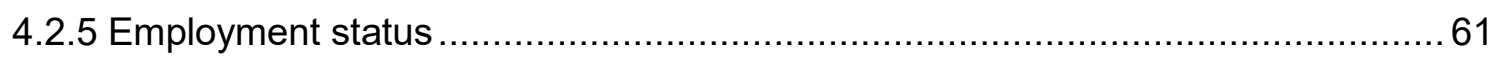

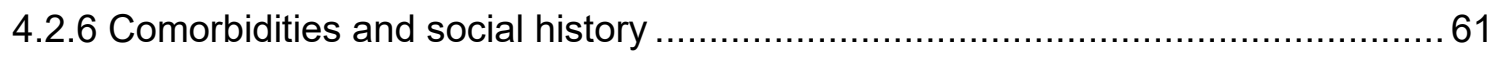

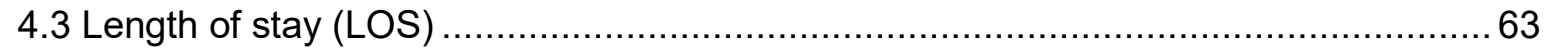

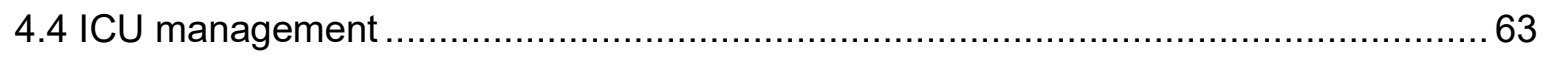

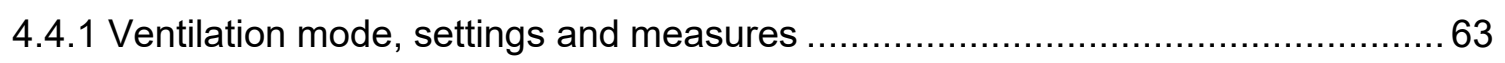

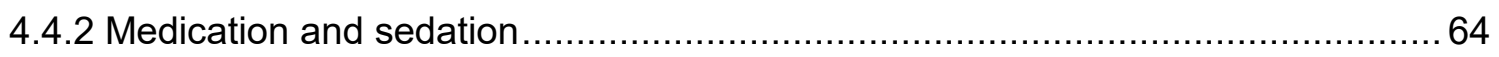


4.5 Ultrasonography of the Respiratory and Peripheral muscles ................................ 65

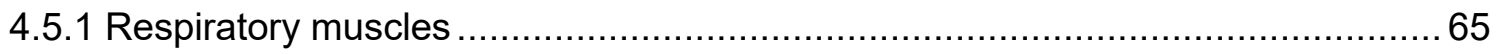

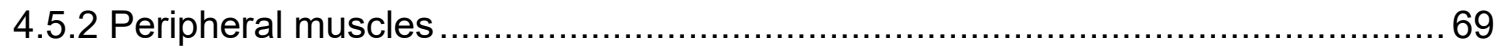

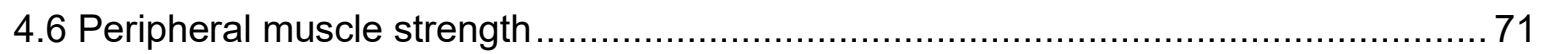

4.6.1 Medical Research Council - Sum Score (MRC-SS) ......................................... 71

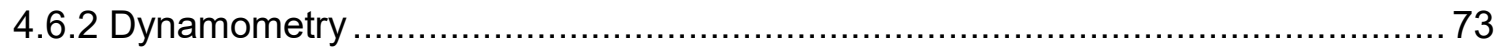

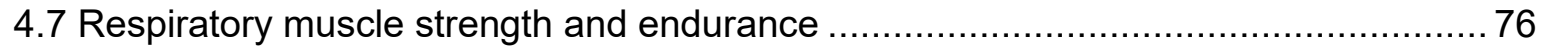

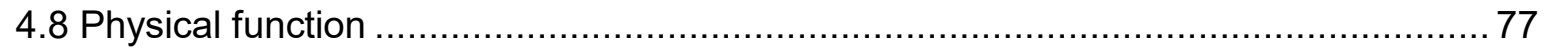

4.8.1 The Chelsea Critical Care Assessment (CPAx) tool and the de Morton Mobility

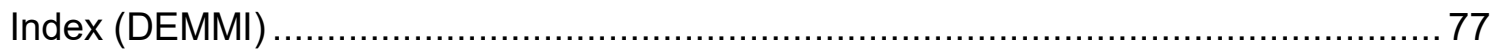

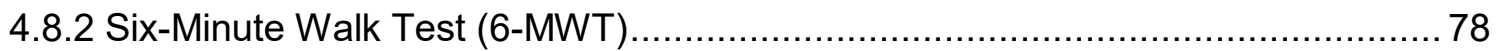

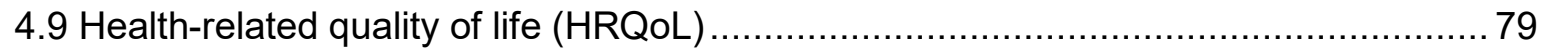

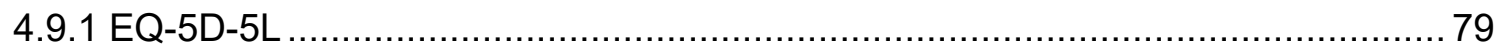

4.10 Correlation between muscle structure, muscle function and physical function ......... 81

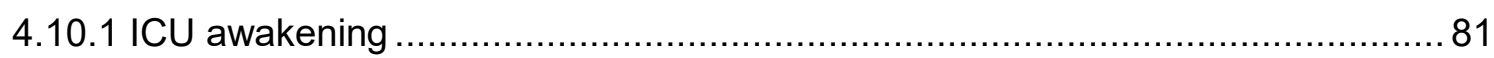

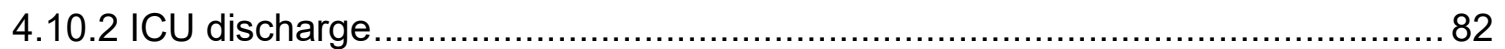

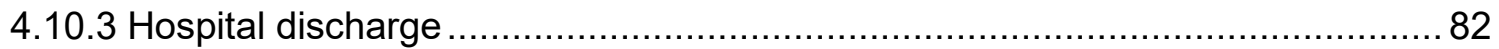

Chapter 5 : Discussion of the Primary Research Study ................................................ 84

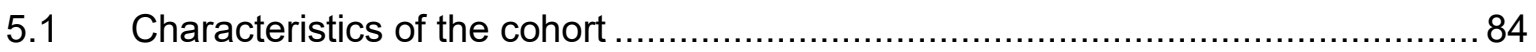

5.2 Outcomes and associations between muscle structure, muscle function and

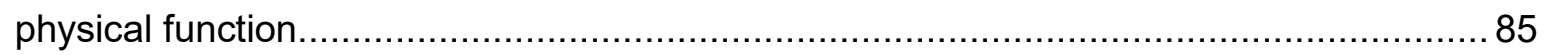

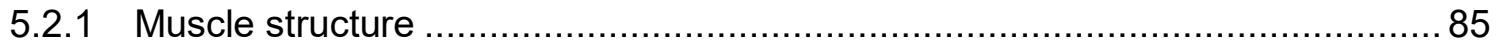

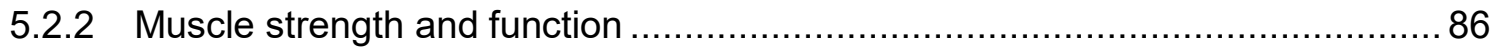

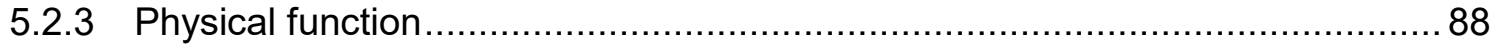

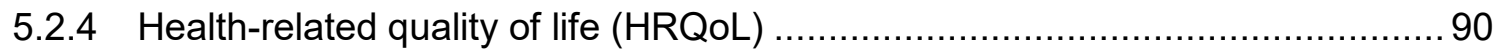




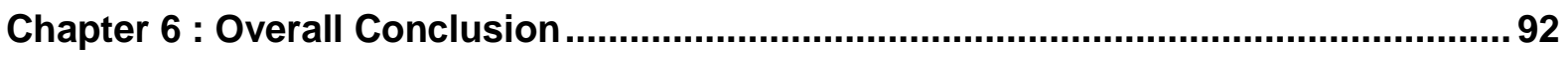

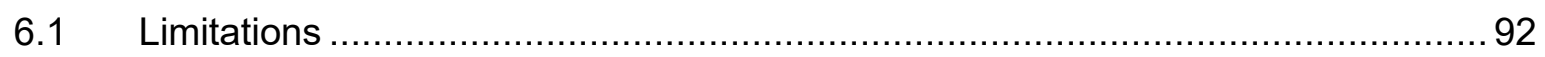

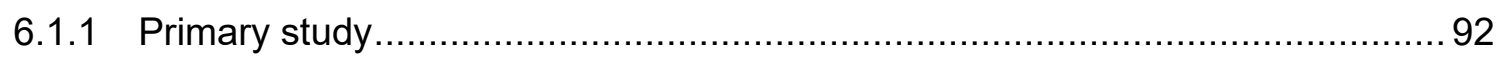

6.2 Recommendation for future research......................................................... 93

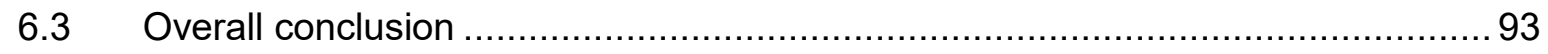

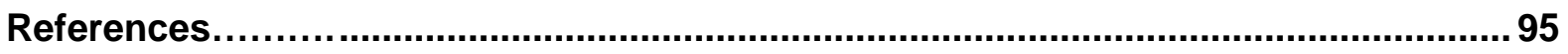

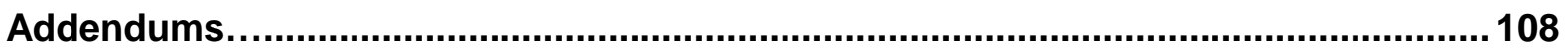

Addendum A: Search Strategies of the Databases Searched ................................ 108

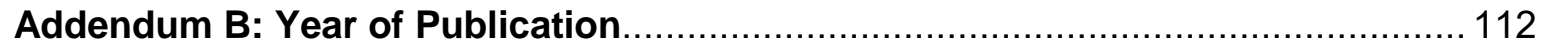

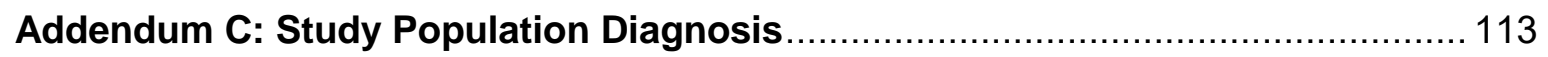

Addendum D: Muscle Studied per Measurement Tool .................................... 114

Addendum E: Testing Procedure of the Measurement Tool ................................ 115

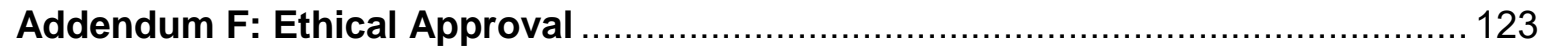

Addendum G: Institutional Permission Letter............................................. 124

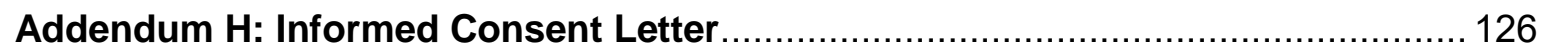

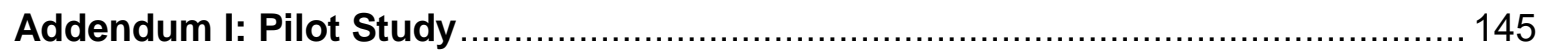

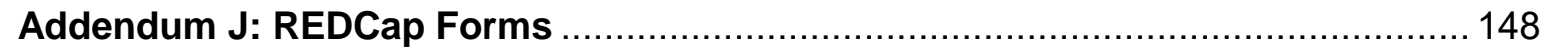

Addendum K: Richmond Agitation and Sedation Scale (RASS) ....................... 169 


\section{LIST OF TABLES}

Table 2.1: Chronological summary of the demographics of the included studies ................ 12

Table 2.2: Chronological summary of type of skeletal muscle and outcome used 15

Table 2.3: Frequency of assessments for measurements of muscle structure and function 18

Table 2.4: Summary of the results for measurements of muscle structure ....................... 25

Table 2.5: Summary of the results for measurements of muscle function......................... 27

Table 2.6: Factors associated with change in muscle structure and function ...................... 29

Table 3.1: Summary of time points for assessments of muscle structure, muscle function,

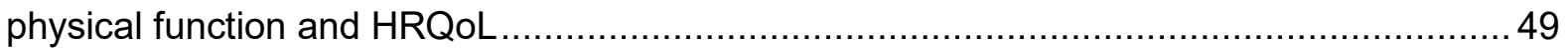

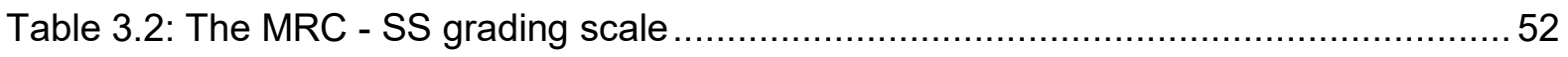

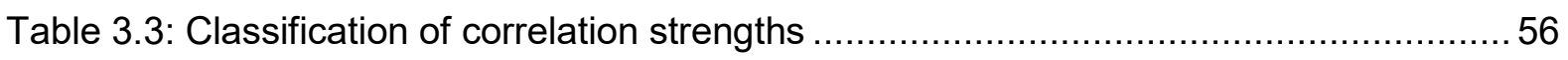

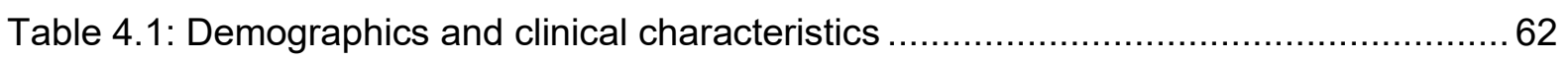

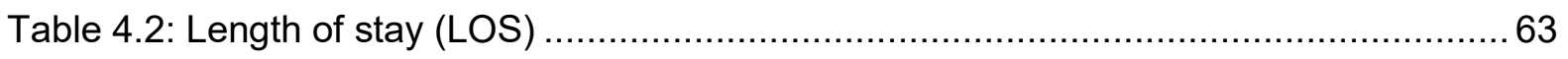

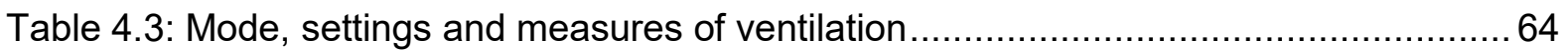

Table 4.4: Number of participants that were exposed to sedation, corticosteroids and insulin

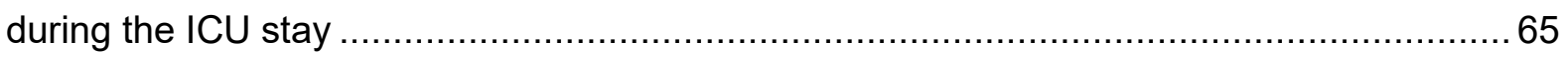

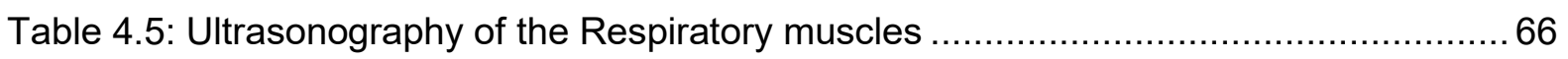

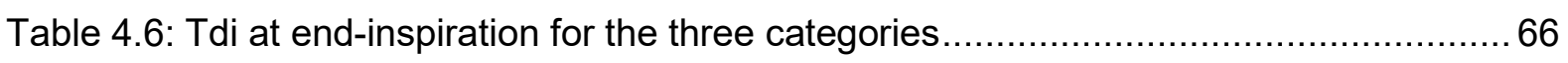

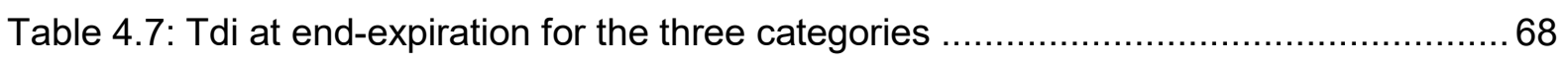

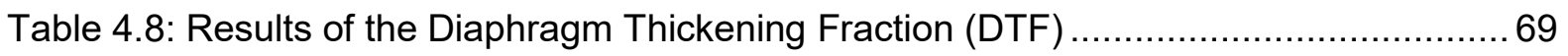

Table 4.9: Ultrasonography of the Peripheral muscles …............................................ 70

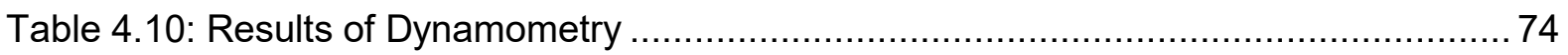

Table 4.11: Normative values for Hand-grip Strength.............................................. 75

Table 4.12: Normative values for Isometric Knee Extension Force................................. 75

Table 4.13: Results of Respiratory muscle strength and endurance ............................. 76

Table 4.14: Normative values for Respiratory muscle strength................................... 77

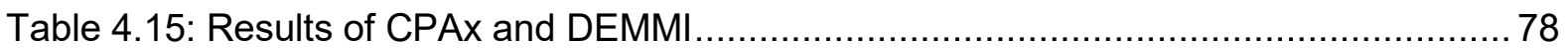


Table 4.16: Results of the 6-MWT

Table 4.17: Normative values for the 6-MWT

Table 4.18: Correlations between muscle structure, muscle function, and physical function at ICU awakening. 81

Table 4.19: Correlations between muscle structure, muscle function, and physical function at ICU discharge 82

Table 4.20: Correlations between muscle structure, muscle function, and physical function at hospital discharge 83 


\section{LIST OF FIGURES}

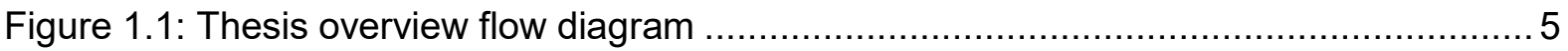

Figure 2.1: Prisma flow diagram of the selection process ............................................ 10

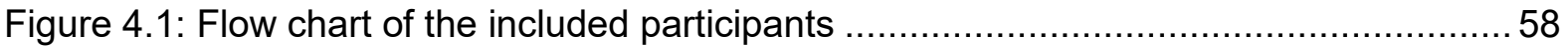

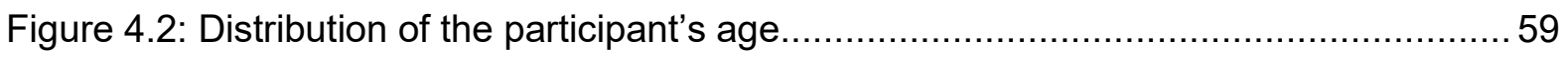

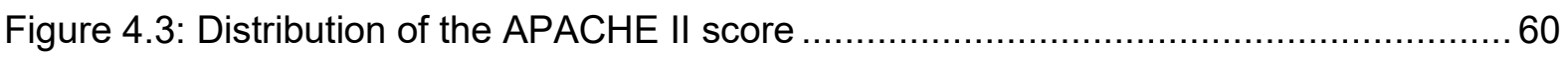

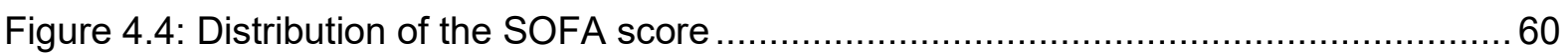

Figure 4.5: Percentage of trauma, medical and surgical admissions ............................... 61

Figure 4.6: Percentage change in Tdi at end-inspiration as compared to the previous day . 67

Figure 4.7: Percentage change in Tdi at end-expiration as compared to the previous day .. 68

Figure 4.8: Decline in the rectus femoris muscle thickness from day one to day three ........70

Figure 4.9: Decline in the rectus femoris muscle CSA from day one to day three............... 71

Figure 4.10: Number of participants that scored 60 on the MRC-SS ............................... 72

Figure 4.11: Percentage of participants with ICU-AW (MRC-SS $<48)$ at the three-time

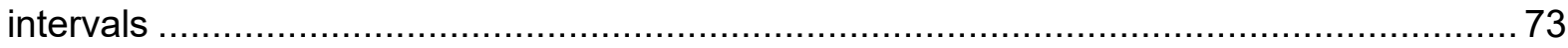

Figure 4.12 : Number of participants that scored for each category of the five dimensions of

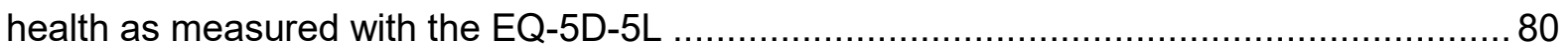




\section{LIST OF ADDENDUMS}

Addendum A: Search Strategies of the Databases Searched........................... 108

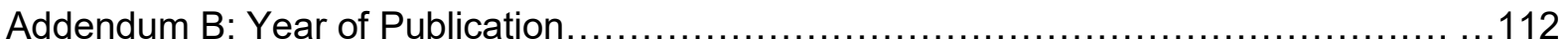

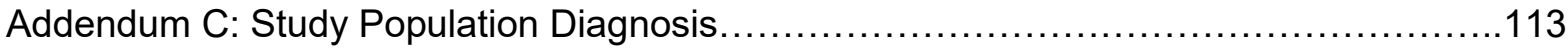

Addendum D: Muscle Studied per Measurement Tool.................................114

Addendum E: Testing Procedure of the Measurement Tools...........................115

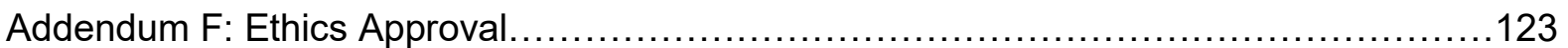

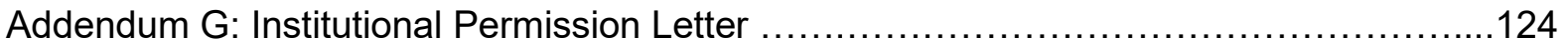

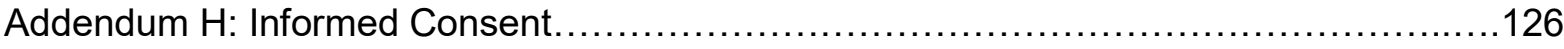

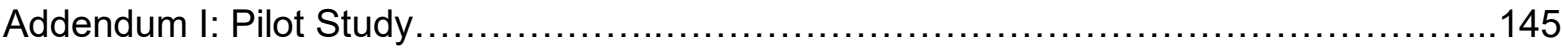

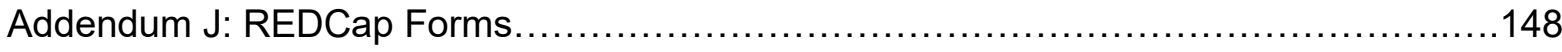

Addendum K: Richmond Agitation and Sedation Scale (RASS) ..........................169 


\section{ABBREVIATIONS}

ADLs: Activities of Daily Living

ALI: Acute Lung Injury

APACHE II: Acute Physiology and Chronic Health Evaluation II

ARF: Acute Respiratory Failure

ARDS: Acute Respiratory Distress Syndrome

ASIS: Anterior Superior lliac Spine

ATS: American Thoracic Society

CPAx: Chelsea Critical Care Physical Assessment

CSA: Cross-Sectional Area

CT: Computed Tomography

DEMMI: de Morton Mobility Index

DTF: Diaphragm Thickening Fraction

ERS: European Respiratory Society

FRC: Functional Residual Capacity

HRQoL: Health-Related Quality of Life

ICU-AW: Intensive Care Unit - Acquired Weakness

ICU: Intensive Care Unit

LOS: Length of Stay

LOV: Length of Ventilation

MIP: Maximum Inspiratory Pressure

MRC-SS: Medical Research Council-Sum Score

mRNA: messenger Ribonucleic Acid

PEEP: Positive End-Expiratory Pressure

PICS: Post-Intensive Care Syndrome

PI: Primary Investigator

PTSD: Post-Traumatic Stress Disorder 
RASS: Richmond Agitation Sedation Scale

RCT: Randomised Control Trials

RF: Rectus Femoris

S5Q: Standardised Five Questions

SOFA: Sequential Organ Failure Assessment

6-MWT: Six-Minute Walk Test

6-MWD: Six-Minute Walk Distance

Tdi: Diaphragm Thickness

VAS: Visual Analogue Scale

VI: Vastus Intermedius

ZOA: Zone of Apposition 


\section{GLOSSARY}

APACHE II: A severity of disease classification system that groups patients according to their risk of death based on physiological data. (1)

Diaphragm Thickness (Tdi): The thickness of the diaphragm muscle at end-expiration (at its thinnest) in the zone of apposition. (2)

Diaphragm Thickening Fraction (DTF): It is used to assess diaphragmatic function and its contribution to respiratory workload and can represent the percentage change in diaphragm thickness during inspiration and expiration. (3)

Dynamometry: Dynamometry is the standard method of volitional muscle force measurement and is designed to assess maximal isometric limb muscle strength objectively. (4)

Immobilisation: Quiet rest in bed for a prolonged period used in the treatment of disease. (5)

Maximal Inspiratory Pressure (MIP): The maximum pressure generated in upper airway, during a voluntary inspiratory effort. $(6,7)$

Positive End-Expiratory Pressure (PEEP): Technique of assisting breathing by increasing the air pressure in the lungs and air passages near the end of expiration so that an increased amount of air remains in the lungs following expiration. (3)

Zone of Apposition (ZOA): The zone of apposition is the area of the chest wall where the abdominal contents reach the lower rib cage. (2) 


\section{Chapter 1: Introduction}

\subsection{Background and study context}

Critical illness is defined as any acute, life-threatening condition requiring vital organ support or intensive monitoring in an intensive care unit (ICU). (8) In the last twenty-five years, intensive care medicine has evolved dramatically in medical technological innovations, resulting in reduced mortality in critically ill patients. While survival of acute phases of critical illness has improved, (8) critically ill survivors have reported experiencing long-term physical function impairments and morbidity that they endure up to five years, effectuating a major socioeconomic impact. $(8,9,10,11)$

Impediments experienced by critically ill survivors include deterioration in strength, physical function and psychological abilities. Signs include reduced joint mobility, reduced ability to perform activities of daily living (ADLs), exercise limitation, delirium, depression and posttraumatic stress disorder (PTSD). "Post-intensive care syndrome" (PICS) has been recognised as the preferred description for this constellation of complications. It is defined as "new or worsening impairments in physical, cognitive or mental health status arising after critical illness and persisting beyond acute care hospitalisation." $(12,13,14)$ Furthermore, it contributes adversely to an individual's quality of life and survival.

The development of skeletal muscle dysfunction in critically ill patients occurs frequently during the ICU stay and can persist beyond ICU and hospital discharge. Muscle strength is defined as the force-generating capacity of a muscle and muscle endurance is defined as the capacity of a muscle or group of muscles to sustain a given task over time and is related to fatigue. (15) Muscle protein homeostasis is defined as the balance between muscle protein synthesis and muscle protein break down. (16) During critical illness, changes in the balance between muscle protein break down and muscle protein synthesis occurs, resulting in a net change in protein turnover. (16) Alterations to this dynamic balance between protein 
synthesis and protein breakdown can result in changes in skeletal muscle mass and can contribute to weakness and fatigue acquired in critically ill patients.

Critical illness affects both the respiratory and peripheral muscles, resulting in muscle atrophy and muscle weakness. (17) Peripheral muscle thickness decreases by approximately $10 \%$ after one week of mechanical ventilation whereas diaphragm atrophy occurs at a rate of $20 \%$ within three to four days of mechanical ventilation. (17) This loss of muscle mass is associated with adverse outcomes such as diaphragm dysfunction, extubation failure, reintubation and prolong mechanical ventilation, intensive care unitacquired weakness (ICU-AW), reduced joint mobility and impaired physical function, as well as associated with an increase in ICU and hospital length of stay (LOS). $(18,19)$ ICU-AW occurs in $25-50 \%$ of critically ill patients and is defined as acute muscle weakness of the extremities and is caused by critical illness. In-hospital risk factors for ICU-AW includes immobilisation, medication such as corticosteroids and neuromuscular blocking agents (NMBA), hyperglycaemia, nutrition and age. $(19,20)$

Impaired physical function is a common finding in critically ill survivors and can persist beyond the first year of surviving from critical illness. Many survivors of critical illness report experiencing impairments in ADLs and exercise limitation. This was reported in a long-term evaluation of 109 Acute Respiratory Distress Syndrome (ARDS) survivors who presented with new or continued impairments in physical function five years after their resolution from critical illness. (21) Participants demonstrated with a persistent reduction in the six-minute walk distance (6-MWD) indicating a continued impairment in exercise endurance as well as a reduced physical quality of life in a patient reported outcome, the 36-Item Short-Form Health Survey. This study by Herridge et al. (21) provided some of the first evidence of longterm physical function impairments after discharge from an ICU. The decline in physical function reported by Herridge et al. (21) was associated with but not limited to immobilisation, sedation, mechanical ventilation, medication and the presence of ICU-AW. 
ICU-AW has been regarded as a cause for impairments in physical function, (22) but evidence supporting this is limited and could be as a result of the lack of research on muscle function as well as the lack of understanding of muscle function in critically ill patients.

It is unknown whether skeletal muscle dysfunction and impaired physical function in critically ill survivors are due to critical illness itself and its ICU treatments and factors or rather to a premorbid composition of critically ill patients, predisposing them to ICU admission and to the type and severity of illness necessitating ICU stay. (8) Due to the uncertainties of the causes for complications endured by critically ill survivors and the societal burden critically ill survivors pose is anticipated to increase, thus the focus of research has recently been shifted. Majority of the research has been focused on studying the impact of critical illness on either muscle function or physical function, the causation of PICS, developing rehabilitation-based interventions to improve functional outcomes or comparing rehabilitation-based interventions in randomised control trials (RCT) to improve functional outcomes in critically ill survivors. $(10,14,20,23)$

Research has mainly been conducted in populations from developed countries. Thus, the known complications critically ill survivors present with that are reported in research studies reflects that of an older population with comorbidities. Conversely, little is known about the trajectory of critically ill patients in developing countries which represents a younger population with few or no comorbidities. Another limitation of some of the research studies was that it only looked at the effects of critical illness on either muscle function or physical function, and not together. $(23,24)$ Furthermore, the long-term efficacy of early mobilisation and rehabilitation in critically ill patients remains to be elucidated.

It is often thought that skeletal muscle dysfunction is associated with impaired physical function, but evidence supporting this hypothesis is limited. Only a few studies looked at the correlation between muscle function and physical function in critically ill survivors. $(25,26)$ In 
a study conducted by Cuthbertson and Goddard (27), they stated that it would be rational that improved muscle-related outcomes would lead to improved cardiorespiratory outcomes, but this may not be so. Although, muscle-related outcomes may improve, cardiorespiratory outcomes may worsen or vice versa leading to adverse events including death. (27) Similarly, it is unknown whether impairments occurring in physical function longitudinally correlate with objective measures of muscle structure and function and has not been comprehensively evaluated in critically ill survivors. Therefore, from a rehabilitation-based intervention perspective, it is unclear what exactly is being treated or whether these rehabilitation-based interventions will improve the outcomes it aims to improve in critically ill survivors.

Hence, this thesis consists of two aims. Firstly, to map the current understanding of muscle structure and muscle function in critically ill patients. And secondly, to investigate and describe outcomes of critically ill patients as well as determine the correlation between muscle structure, muscle function and physical function in critically ill patients at ICU discharge and hospital discharge in a South African population setting.

\subsection{Thesis overview}

This thesis consists of six chapters. Chapter 2 is written as a research article according to the BioMed Central submission guidelines and the PRISMA extension for scoping reviews (PRISMA-ScR) guidelines for the Critical Care journal. The search strategy for the scoping review will be updated prior to submitting the review for publication. The findings from the scoping review (Chapter 2) assisted in achieving the second aim of this thesis through a primary study (Chapter 3, Chapter 4, Chapter 5 and Chapter 6) (Figure 1.1). One reference list is presented for this thesis and individual reference lists will be prepared for each article with submission for publication. 


\section{Chapter 1: Introduction}

Background, Study Context and Thesis Outline

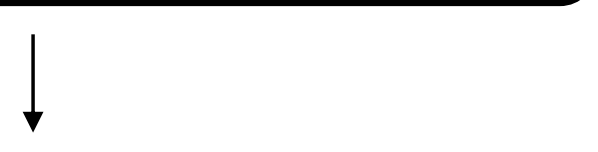

\section{Chapter 2: Scoping Review}

Current Understanding of Muscle Structure and

Muscle Function in Critically III Patients

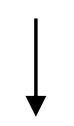

Chapter 3: Methodology of Primary Research Study

$\downarrow$

Chapter 4: Results of Primary Research Study

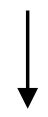

Chapter 5: Discussion of Primary Research Study

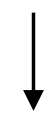

Chapter 6: Overall Conclusion

Figure 1.1: Thesis overview flow diagram 


\title{
Chapter 2: Scoping Review
}

Current Understanding of Muscle Structure and Muscle Function in Critically III Patients:

\author{
A Scoping Review
}

\subsection{Background}

Critical illness is a life-threatening multisystem process that results in morbidity and mortality. However, recent improvements in critical care and ventilator management has led to a significant reduction in mortality and an increase in critically ill survivors. Despite this success, critically ill survivors report experiencing a lasting sequalae such as reduced physical function, cognitive and mental impairments and a reduced health-related quality of life (HRQoL). (28) Reduced physical function in critically ill survivors has led to the skeletal muscle system being postulated as one of the causes of this impairment. (22) Skeletal muscle dysfunction, in particular a loss of muscle mass and weakness, is commonly seen in the respiratory and peripheral muscles. Muscle atrophy and weakness of the respiratory and peripheral muscles occurs in $25-50 \%$ of critically ill patients and presents early during the management of acute illness and can persist months or years post-hospital discharge. (17)

In health, muscle mass relies on the balance between protein synthesis and protein degradation and any alteration to this balance is associated with skeletal muscle dysfunction. During critical illness, an increase in the rate of muscle protein break down occurs and since critically ill patients portray anabolic resistance, this may result in a fall in muscle protein synthesis. (16) This imbalance between protein synthesis and protein degradation results in changes in skeletal muscle mass in critically ill patients. (16) This imbalance between protein synthesis and protein degradation can be triggered by various factors, notably illness severity, systemic inflammation, immobilisation, medication or malnutrition, but the clinical significance of these different factors on muscle structure and function is poorly understood. 
Skeletal muscle is a highly organised tissue of muscle fibres and connective tissue and contributes to biological functions. (29) The respiratory and peripheral muscles are made up slow twitch (Type I) and fast twitch (Type II) muscle fibres. Type I muscle fibres utilises aerobic respiration and contracts relatively slowly and is used in endurance whereas, Type II muscle fibres primarily uses anaerobic respiration for fast contraction and used for fast burst of activity. (30) The change in fibre type composition may be responsible for impairments seen in critically ill patients who are weakened due to prolonged inactivity. However, it is unclear whether in both respiratory and peripheral muscles, the composition and contractility of muscle fibre types are equally affected or not. (30)

Research studies have used a variety of measurement tools to assess and observe muscle structure and function in critically ill patients. Some of these measurement tools include ultrasonography, muscle biopsies, the Medical Research Council-Sum Score (MRC-SS), dynamometry, bilateral phrenic nerve stimulation and electrophysiological measurements. However, it is not known how frequently these measurement tools are being used, when they are being performed during the patient's hospital stay and the methodology of these measurement tools.

Given the impact critical illness has on skeletal muscles, the focus of research has recently been shifted towards studying muscle structure and function in critically ill patients. Therefore, the purpose of this scoping review was to map the current literature reporting on muscle structure and function in critically ill patients in order to:

(i) Describe the populations where muscle structure and function have been investigated

(ii) Describe the measurement tools used to assess muscle structure and function

(iii) Determine the frequency of assessments within the disease trajectory

(iv) Describe the testing procedure of the different type of measurement tools

(v) Describe the changes in muscle structure and function in critically ill patients 
(vi) Discuss the factors associated with change in muscle structure and function

\subsection{Methods}

A scoping review was conducted and guided by the framework published by Arksey and O'Malley (31) and written according to the PRISMA extension for scoping reviews (PRISMA$\mathrm{ScR}$ ) guidelines to map the current literature reporting on muscle structure and function in critically ill patients.

\subsubsection{Eligibility criteria}

The inclusion and exclusion criteria were developed as the research team became familiar with the literature. Articles were eligible for inclusion if research 1) was conducted on adults 18 years+; 2) investigated muscle structure and function in critically ill patients; and 3) was conducted as a primary study. Articles were excluded if 1) the research was conducted on animals; 2) the article was a synthesis of existing research e.g., reviews; or opinion e.g. commentary; and 3) articles were not available in English.

\subsubsection{Information sources and search strategy}

A systematic literature search was conducted on the following six computerised bibliographic databases through the Stellenbosch University Library namely; EBSCOhost - Cinahl, EBSCOhost - Medline, Pubmed, Science direct, Scopus and Web of Science. The search was done in August 2019. Search strategies for each database were developed according to the function of each respective database. MeSH terms and limits were used where applicable. A detailed search strategy for each database can be found in Addendum A.

\subsubsection{Selection of sources of evidence}

The primary investigator (PI) and a secondary independent reviewer (HP) systematically screened and evaluated all titles, abstracts and full-text articles returned by the search strategy for relevance and inclusion to the scoping review. In the event of disagreements, a meeting was arranged between the two reviewers to reach consensus. If consensus could not be reached, a third reviewer (ALS) was consulted. 


\subsubsection{Data charting process and data items}

The PI independently extracted and recorded all relevant data from the included papers, using a Microsoft Excel spreadsheet. Data extracted and recorded included: author(s), year of publication, title, journal, country of origin, research design, study population, sample age, sample size, type of skeletal muscles (respiratory, peripheral, postural), type of measurement tools used, methodology and findings.

\subsubsection{Synthesis of results}

Key findings regarding muscle structure and function, the type of measurement tool used, factors associated with change in muscle structure and function, the frequency of assessments and the testing procedure of the measurement tools were extracted from the results sections of the included articles and summarised and reported in different sections under results.

\subsection{Results}

\subsubsection{Selection of sources of evidence}

The search strategy yielded a total of 1282 studies. The Prisma flow diagram (Figure 2.1) illustrates the selection process at title, abstract and at full-text level. A total number of 34 studies are included in this scoping review. 


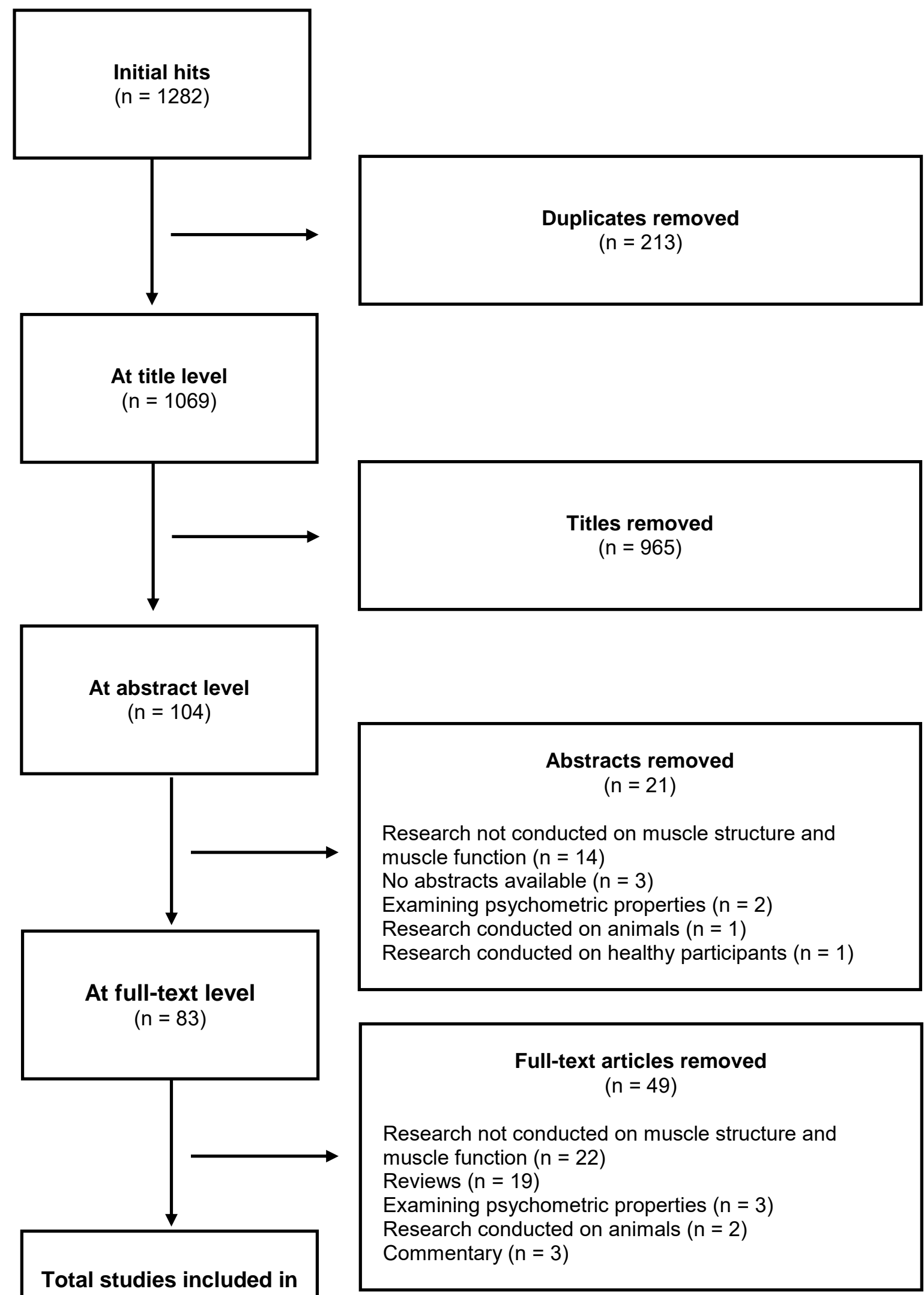
the review

$(n=34)$

Figure 2.1: Prisma flow diagram of the selection process 


\subsubsection{Authors, year of publication, country of origin, sample size and sample population}

All 34 studies were conducted in developed countries from four continents with majority of the studies being conducted in Europe $(n=26)$ (Table 2.1). None of the included studies were conducted in countries from Africa, South America or any developing countries. The common research designs were cohort studies (Table 2.1).

The studies included in this scoping review were published between 2005 and 2018, with majority being published in 2013 and 2015 (Addendum B). Limited research on muscle structure and function was published thereafter. Populations investigated included sepsis $(n=17,50 \%)$, trauma $(n=10,29.4 \%)$, pneumonia $(n=8,23.5 \%)$, acute respiratory distress syndrome (ARDS) $(n=6,17.6 \%)$ and septic shock $(n=6,17.6 \%)$. The list of "other" diagnosis is represented in Addendum C. 
Stellenbosch University https://scholar.sun.ac.za

Table 2.1: Chronological summary of the demographics of the included studies

\begin{tabular}{|c|c|c|c|c|c|c|c|c|c|c|c|c|}
\hline \multirow[b]{2}{*}{ Author(s) } & \multirow[b]{2}{*}{ Year } & \multirow[b]{2}{*}{$\begin{array}{l}\text { Research Design/ } \\
\text { Article Type }\end{array}$} & \multicolumn{3}{|c|}{ Country of Origin } & \multirow[b]{2}{*}{$\begin{array}{c}\text { Sample } \\
\text { Size }\end{array}$} & \multicolumn{6}{|c|}{ Study Population } \\
\hline & & & Europe & $\begin{array}{c}\text { North } \\
\text { America }\end{array}$ & $\begin{array}{l}\text { Asia } \\
\text { Pacific }\end{array}$ & & Sepsis & Trauma & Pneumonia & ARDS & $\begin{array}{l}\text { Septic } \\
\text { shock }\end{array}$ & Other \\
\hline Buscher et al. (32) & 2005 & Clinical trail & Germany & & & 16 & & & $\checkmark$ & $\checkmark$ & & $\checkmark$ \\
\hline Ginz et al. (33) & 2005 & Cohort & Switzerland & & & 19 & $\checkmark$ & & $\checkmark$ & $\checkmark$ & & $\checkmark$ \\
\hline Klaude et al. (34) & 2005 & Cohort & Sweden & & & 7 & $\checkmark$ & & & & & $\checkmark$ \\
\hline Fredriksson et al. (35) & 2006 & Cohort & Sweden & & & 10 & & & & & & $\checkmark$ \\
\hline De Jonghe et al. (24) & 2007 & Cohort & France & & & 116 & & $\checkmark$ & $\checkmark$ & & & $\checkmark$ \\
\hline Ginz et al. (36) & 2008 & Case studies & Switzerland & & & 8 & $\checkmark$ & & $\checkmark$ & & & $\checkmark$ \\
\hline $\begin{array}{c}\text { Weber-Carstens et al. } \\
(37)\end{array}$ & 2009 & Cohort & Germany & & & 56 & & & $\checkmark$ & & & $\checkmark$ \\
\hline Hermans et al. (38) & 2010 & Cohort & Belgium & & & 10 & & & & & $\checkmark$ & $\checkmark$ \\
\hline Jaber et al. (39) & 2011 & Cohort & France & & & 37 & & $\checkmark$ & & & & $\checkmark$ \\
\hline Bierbrauer et al. (40) & 2012 & Cohort & Germany & & & 24 & $\checkmark$ & $\checkmark$ & & $\checkmark$ & & $\checkmark$ \\
\hline Derde et al. (41) & 2012 & Clinical trail & $\begin{array}{l}\text { Belgium \& } \\
\text { Sweden }\end{array}$ & & & 208 & & & & & & $\checkmark$ \\
\hline Llano-Diez et al. (42) & 2012 & Cohort & Sweden & & & 7 & & & & & & $\checkmark$ \\
\hline Cartwright et al. (43) & 2013 & Pilot study & & USA & & 16 & & & $\checkmark$ & & & $\checkmark$ \\
\hline Demoule et al. (44) & 2013 & Cohort & France & & & 85 & & & & & $\checkmark$ & $\checkmark$ \\
\hline Puthucheary et al. (45) & 2013 & Cohort & United Kingdom & & & 63 & $\checkmark$ & $\checkmark$ & & & & $\checkmark$ \\
\hline $\begin{array}{l}\text { Supinski and Callahan } \\
(46)\end{array}$ & 2013 & Cohort & & USA & & 57 & & & & & & $\checkmark$ \\
\hline $\begin{array}{c}\text { Weber-Carstens et al. } \\
(47)\end{array}$ & 2013 & Cohort & Germany & & & 18 & $\checkmark$ & $\checkmark$ & & $\checkmark$ & & $\checkmark$ \\
\hline $\begin{array}{l}\text { Baldwin and Bersten } \\
(23)\end{array}$ & 2014 & Cross sectional & & & Australia & 16 & $\checkmark$ & & & & $\checkmark$ & \\
\hline Sheean et al. (48) & 2014 & Cross sectional & & USA & & 56 & $\checkmark$ & & & & & $\checkmark$ \\
\hline Wollersheim et al. (49) & 2014 & Cohort & Germany & & & 29 & $\checkmark$ & $\checkmark$ & & $\checkmark$ & & $\checkmark$ \\
\hline
\end{tabular}


Table 2.1: Continued

\begin{tabular}{|c|c|c|c|c|c|c|c|c|c|c|c|c|}
\hline \multirow[b]{2}{*}{ Author(s) } & \multirow[b]{2}{*}{ Year } & \multirow[b]{2}{*}{$\begin{array}{l}\text { Research Design/ } \\
\text { Article Type }\end{array}$} & \multicolumn{3}{|c|}{ Country of Origin } & \multirow[b]{2}{*}{$\begin{array}{l}\text { Sample } \\
\text { Size }\end{array}$} & \multicolumn{6}{|c|}{ Study Population } \\
\hline & & & Europe & $\begin{array}{c}\text { North } \\
\text { America }\end{array}$ & $\begin{array}{l}\text { Asia } \\
\text { Pacific }\end{array}$ & & Sepsis & Trauma & Pneumonia & ARDS & $\begin{array}{l}\text { Septic } \\
\text { shock }\end{array}$ & Other \\
\hline Bissett (50) & 2015 & Cohort & & & Australia & 43 & $\checkmark$ & & $\checkmark$ & & & $\checkmark$ \\
\hline Goligher et al. (3) & 2015 & Cohort & & Canada & & 107 & $\checkmark$ & & & & & $\checkmark$ \\
\hline Hooijman et al. (51) & 2015 & Cohort & Netherlands & & & 22 & $\checkmark$ & $\checkmark$ & & & $\checkmark$ & $\checkmark$ \\
\hline Puthucheary et al. (52) & 2015 & Cohort & United Kingdom & & Singapore & 30 & $\checkmark$ & $\checkmark$ & & & & $\checkmark$ \\
\hline Schepens et al. (53) & 2015 & Cohort & Belgium & & & 53 & & & & & & $\checkmark$ \\
\hline Umbrello et al. (54) & 2015 & Pilot clinical study & Italy & & & 25 & & & & & & $\checkmark$ \\
\hline Demoule et al. (55) & 2016 & Cohort & France & & & 42 & & & & & $\checkmark$ & $\checkmark$ \\
\hline Lee et al. (56) & 2016 & Cohort & & & Korea & 13 & & & $\checkmark$ & & & $\checkmark$ \\
\hline Turton et al. (57) & 2016 & Cohort & United Kingdom & & & 47 & $\checkmark$ & & & & & $\checkmark$ \\
\hline Zambon et al. (58) & 2016 & Cohort & Italy & & & 40 & $\checkmark$ & $\checkmark$ & & $\checkmark$ & $\checkmark$ & $\checkmark$ \\
\hline Van den Berg et al. (59) & 2017 & Cohort & Netherlands & & & 49 & & & & & & $\checkmark$ \\
\hline Goligher et al. (60) & 2018 & Cohort & & Canada & & 191 & $\checkmark$ & & & & & $\checkmark$ \\
\hline $\begin{array}{c}\text { Hernandez-Scorro et al. } \\
(61)\end{array}$ & 2018 & Cohort & Spain & & & 48 & & & & & & $\checkmark$ \\
\hline Puthucheary et al. (62) & 2018 & Cohort & United Kingdom & & & 63 & $\checkmark$ & $\checkmark$ & & & & $\checkmark$ \\
\hline
\end{tabular}

KEY: $\checkmark$-Yes; USA - United States of America; ARDS - Acute Respiratory Distress Syndrome 


\subsubsection{Type of skeletal muscle and the different type of measurement tools}

Research included respiratory, peripheral and postural muscles (Table 2.2). Fourteen studies $(41 \%)$ investigated only the respiratory muscles, 13 studies $(38 \%)$ investigated only the peripheral muscles, five studies $(15 \%)$ investigated both the respiratory and peripheral muscles and two studies $(6 \%)$ included both peripheral and postural muscles. The diaphragm and the vastus lateralis muscle were the two muscle groups most often included (Addendum D). The measurement tools used to assess muscle structure and function, and the outcomes assessed are shown in Table 2.2. 
Table 2.2: Chronological summary of type of skeletal muscle and outcome used

\begin{tabular}{|c|c|c|c|c|c|c|c|c|c|c|c|c|c|}
\hline \multirow{3}{*}{ Author(s) } & \multirow{3}{*}{ Year } & \multicolumn{12}{|c|}{ Type of outcome used } \\
\hline & & \multicolumn{3}{|c|}{ Muscle structure } & \multicolumn{9}{|c|}{ Muscle function } \\
\hline & & $\begin{array}{l}\text { Ultrasono- } \\
\text { graphy }\end{array}$ & $\begin{array}{l}\text { Muscle } \\
\text { biopsy }\end{array}$ & $\begin{array}{l}\text { CT } \\
\text { Scan }\end{array}$ & $\begin{array}{c}\text { Bilateral } \\
\text { phrenic nerve } \\
\text { stimulation } \\
\end{array}$ & $\begin{array}{c}\text { Trans- } \\
\text { diaphragmatic } \\
\text { pressure }\end{array}$ & $\begin{array}{l}\text { Endotracheal } \\
\text { pressure }\end{array}$ & MIP & MEP & MRC-SS & Dynamometry & $\begin{array}{c}\text { Electro- } \\
\text { physiological } \\
\text { measurements }\end{array}$ & $\begin{array}{c}\text { Muscle torque } \\
\text { via nerve } \\
\text { stimulation } \\
\end{array}$ \\
\hline Buscher et al. (32) & 2005 & & & & $\mathrm{R}$ & $\mathrm{R}$ & $\mathrm{R}$ & & & & & & \\
\hline Ginz et al. (33) & 2005 & & & & & & & & & & & & $\begin{array}{c}\text { P: PT, PTD, } \\
\text { PTd, CT, HRT }\end{array}$ \\
\hline Klaude et al. (34) & 2005 & & $P$ & & & & & & & & & & \\
\hline Fredriksson et al. (35) & 2006 & & $\begin{array}{l}\mathrm{R} \\
\mathrm{P}\end{array}$ & & & & & & & & & & \\
\hline De Jonghe et al. (24) & 2007 & & & & & & & $\mathrm{R}$ & $\mathrm{R}$ & $P$ & & & \\
\hline Ginz et al. (36) & 2008 & & & & & & & & & & & & $\begin{array}{l}\text { P: PT, PTD, } \\
\text { PTd, CT }\end{array}$ \\
\hline $\begin{array}{c}\text { Weber-Carstens et al. } \\
(37)\end{array}$ & 2009 & & & & & & & & & $P$ & & $P$ & \\
\hline Hermans et al. (38) & 2010 & & & & $\mathrm{R}$ & $\mathrm{R}$ & & & & & & & \\
\hline Jaber et al. (39) & 2011 & & $\mathrm{R}$ & & $\mathrm{R}$ & & $\mathrm{R}$ & & & & & & \\
\hline Bierbrauer et al. (40) & 2012 & & $P$ & & & & & & & $P$ & & $P$ & \\
\hline Derde et al. (41) & 2012 & & $\begin{array}{c}\mathrm{P} \\
\mathrm{PST} \\
\end{array}$ & & & & & & & & & & \\
\hline Llano-Diez et al. (42) & 2012 & P: CSA & $\mathrm{P}$ & & & & & & & & & $P$ & \\
\hline Cartwright et al. (43) & 2013 & $\begin{array}{c}\text { R: Tdi } \\
\text { P: MT, Ec }\end{array}$ & & & & & & & & & & & \\
\hline Demoule et al. (44) & 2013 & & & & $\mathrm{R}$ & & $\mathrm{R}$ & & & & & & \\
\hline Puthucheary et al. (45) & 2013 & P: CSA & $P$ & & & & & & & & & & \\
\hline $\begin{array}{l}\text { Supinski and Callahan } \\
(46)\end{array}$ & 2013 & & & & $\mathrm{R}$ & $\mathrm{R}$ & & & & & & & \\
\hline $\begin{array}{l}\text { Weber-Carstens et al. } \\
(47)\end{array}$ & 2013 & & $P$ & & & & & & & & & $P$ & \\
\hline $\begin{array}{l}\text { Baldwin and Bersten } \\
\text { (23) }\end{array}$ & 2014 & $\begin{array}{l}\text { R: Tdi } \\
\text { P: MT }\end{array}$ & & & & & & $\mathrm{R}$ & & $P$ & $P$ & & \\
\hline Sheean et al. (48) & 2014 & & & PST & & & & & & & & & \\
\hline Wollersheim et al. (49) & 2014 & & $\mathrm{P}$ & & & & & & & $\mathrm{P}$ & & & \\
\hline
\end{tabular}


Table 2.2: Continued

\begin{tabular}{|c|c|c|c|c|c|c|c|c|c|c|c|c|c|}
\hline \multirow{3}{*}{ Author(s) } & \multirow{3}{*}{ Year } & \multicolumn{12}{|c|}{ Type of outcome used } \\
\hline & & \multicolumn{3}{|c|}{ Muscle structure } & \multicolumn{9}{|c|}{ Muscle function } \\
\hline & & $\begin{array}{l}\text { Ultrasono- } \\
\text { graphy }\end{array}$ & $\begin{array}{l}\text { Muscle } \\
\text { biopsy }\end{array}$ & $\begin{array}{l}\text { CT } \\
\text { Scan }\end{array}$ & $\begin{array}{c}\text { Bilateral } \\
\text { phrenic nerve } \\
\text { stimulation }\end{array}$ & $\begin{array}{c}\text { Trans- } \\
\text { diaphragmatic } \\
\text { pressure }\end{array}$ & $\begin{array}{l}\text { Endotracheal } \\
\text { pressure }\end{array}$ & MIP & MEP & MRC-SS & Dynamometry & $\begin{array}{c}\text { Electro- } \\
\text { physiological } \\
\text { measurements }\end{array}$ & $\begin{array}{l}\text { Muscle torque } \\
\text { via nerve } \\
\text { stimulation }\end{array}$ \\
\hline Bissett (50) & 2015 & & & & & & & $\mathrm{R}$ & & & & & \\
\hline Goligher et al. (3) & 2015 & R: Tdi & & & & & & & & & & & \\
\hline Hooijman et al. (51) & 2015 & & $\begin{array}{l}R \\
P\end{array}$ & & & & & & & & & & \\
\hline Puthucheary et al. (52) & 2015 & $\begin{array}{c}\text { P: CSA, } \\
\text { Ec }\end{array}$ & $P$ & & & & & & & & & & \\
\hline Schepens et al. (53) & 2015 & R: Tdi & & & & & & & & & & & \\
\hline Umbrello et al. (54) & 2015 & $\begin{array}{c}\text { R:Tdi, } \\
\text { DTF, Ex }\end{array}$ & & & $\mathrm{R}$ & $\mathrm{R}$ & & & & & & & \\
\hline Demoule et al. (55) & 2016 & & & & $\mathrm{R}$ & & $\mathrm{R}$ & & & & & & \\
\hline Lee et al. (56) & 2016 & & & $\mathrm{R}$ & & & & & & & & & \\
\hline Turton et al. (57) & 2016 & P: MT & & & & & & & & & & & \\
\hline Zambon et al. (58) & 2016 & $\begin{array}{l}\text { R:Tdi, } \\
\text { DTF }\end{array}$ & & & & & & & & & & & \\
\hline $\begin{array}{c}\text { Van den Berg et al. } \\
(59)\end{array}$ & 2017 & & $\mathrm{R}$ & & & & & & & & & & \\
\hline Goligher et al. (60) & 2018 & $\begin{array}{l}\text { R: Tdi, } \\
\text { DTF }\end{array}$ & & & & & & & & & & & \\
\hline $\begin{array}{c}\text { Hernandez-Scorro et } \\
\text { al. (61) }\end{array}$ & 2018 & $\begin{array}{c}\text { P:MT, } \\
\text { CSA, Ec }\end{array}$ & & & & & & & & & & & \\
\hline Puthucheary et al. (62) & 2018 & & $\mathrm{P}$ & & & & & & & & & & \\
\hline
\end{tabular}

KEY: R - Respiratory; P - Peripheral; PST - Postural; MT - Muscle Thickness; MIP - Maximum Inspiratory Pressure; MEP - Maximum Expiratory Pressure;

MRC-SS - Medical Research Council Sum - Score; Ec - Echogenicity; CSA - Cross Sectional Area; Tdi - Diaphragm thickness; DTF - Diaphragm Thickening Fraction, Ex - Excursion; HRT - Half-Relaxation Time; PT - Peak Torque; PTD - Peak Torque Development; PTd - Peak Torque decay; CT - Contraction Time 


\subsubsection{Frequency of assessments}

Majority $(76 \%)$ of the measurements for both muscle structure and function were conducted during the patients' ICU stay (Table 2.3). Only one study followed up with the patients at two, four- and six-months post-hospital discharge (23). None of the other 33 studies reported whether patients were followed up and if measurements were taken at ICU discharge, during the patient's ward stay, at hospital discharge or post-hospital discharge. There were also 14 studies that reported the frequency of assessments differently and are found under the heading "other" in Table 2.3. 
Table 2.3: Frequency of assessments for measurements of muscle structure and function

\begin{tabular}{|c|c|c|c|c|c|c|}
\hline \multirow{3}{*}{ Author(s) } & \multicolumn{6}{|c|}{ Frequency of Assessments } \\
\hline & \multicolumn{5}{|c|}{ ICU Stay } & \multirow{2}{*}{$\begin{array}{c}\text { Post-hospital } \\
\text { Discharge }\end{array}$} \\
\hline & Baseline & $\begin{array}{c}\text { Day } \\
\text { interval }\end{array}$ & $\begin{array}{c}\text { Hour } \\
\text { interval }\end{array}$ & Other & $\begin{array}{c}\text { Not } \\
\text { Reported }\end{array}$ & \\
\hline \multicolumn{7}{|c|}{ Respiratory: Structure } \\
\hline \multicolumn{7}{|c|}{ Ultrasonography } \\
\hline Baldwin and Bersten (23) & & & & & $\checkmark$ & \\
\hline Cartwright et al. (43) & $\checkmark$ & $3 ; 7 ; 14$ & & & & $2 ; 4 ; 6$ \\
\hline Goligher et al. (3) & & & & $\begin{array}{l}\text { Daily on week days until } \\
\text { extubation or Day } 14\end{array}$ & & \\
\hline Goligher et al. (60) & & & & $\begin{array}{l}\text { Daily on week days until } \\
\text { extubation or Day } 14\end{array}$ & & \\
\hline Schepens et al. (53) & $\checkmark$ & 5 & & & & \\
\hline Umbrello et al. (54) & & & & & $\checkmark$ & \\
\hline Zambon et al. (58) & & & & & $\checkmark$ & \\
\hline \multicolumn{7}{|c|}{ Muscle biopsy } \\
\hline Fredriksson et al. (35) & & & & & $\checkmark$ & \\
\hline Jaber et al. (39) & & & & $\begin{array}{c}\text { After two, three and } 24 \text { hours } \\
\text { of MV }\end{array}$ & & \\
\hline Hooijman et al. (51) & & & & & $\checkmark$ & \\
\hline Van den Berg et al. (59) & & & & Only after 17 hours of MV & & \\
\hline \multicolumn{7}{|c|}{ CT Scan } \\
\hline Lee et al. (56) & & & & & $\checkmark$ & \\
\hline \multicolumn{7}{|c|}{ Respiratory: Function } \\
\hline \multicolumn{7}{|c|}{ Trans-diaphragmatic pressure/Endotracheal pressure via bilateral phrenic nerve stimulation } \\
\hline Buscher et al. (32) & & & & $\begin{array}{l}\text { Before extubation and/or after } \\
\text { failing at least one extubation }\end{array}$ & & \\
\hline Demoule et al. (44) & & $1 ; 3$ & & & & \\
\hline Demoule et al. (55) & & & $\begin{array}{c}24 ; 48 \\
72\end{array}$ & & & \\
\hline Hermans et al. (38) & & & & $\begin{array}{c}\text { Two to three days after ICU } \\
\text { admission } \\
\end{array}$ & & \\
\hline Jaber et al. (39) & & & $24 ; 36$ & & & \\
\hline Supinski and Callahan (46) & & & & & $\checkmark$ & \\
\hline Umbrello et al. (54) & & & & & $\checkmark$ & \\
\hline \multicolumn{7}{|c|}{ MIP \& MEP } \\
\hline Baldwin and Bersten (23) & & & & & $\checkmark$ & \\
\hline Bissett (50) & & & & $\begin{array}{c}\text { After } 48 \text { hours of successful } \\
\text { extubation }\end{array}$ & & \\
\hline De Jonghe et al. (24) & & & & Day of awakening & & \\
\hline \multicolumn{7}{|c|}{ Peripheral: Structure } \\
\hline \multicolumn{7}{|c|}{ Ultrasonography } \\
\hline Baldwin and Bersten (23) & & & & & $\checkmark$ & \\
\hline Cartwright et al. (43) & $\checkmark$ & $3 ; 7 ; 14$ & & & & $2 ; 4 ; 6$ \\
\hline Hernandez-Scorro et al. (61) & & & & & $\checkmark$ & \\
\hline Llano-diez et al. (42) & & & & For seven to 11 days & & \\
\hline Puthucheary et al. (45) & & $1 ; 3 ; 7 ; 10$ & & & & \\
\hline Puthucheary et al. (52) & & $1 ; 10$ & & & & \\
\hline Turton et al. (99) & & $1 ; 5 ; 10$ & & & & \\
\hline
\end{tabular}


Table 2.3: Continued

\begin{tabular}{|c|c|c|c|c|c|c|}
\hline \multirow{3}{*}{ Author(s) } & \multicolumn{6}{|c|}{ Frequency of Assessments } \\
\hline & \multicolumn{5}{|c|}{ ICU Stay } & \multirow{2}{*}{$\begin{array}{c}\text { Post-hospital } \\
\text { Discharge }\end{array}$} \\
\hline & Baseline & $\begin{array}{c}\text { Day } \\
\text { interval }\end{array}$ & $\begin{array}{c}\text { Hour } \\
\text { interval }\end{array}$ & Other & $\begin{array}{c}\text { Not } \\
\text { Reported }\end{array}$ & \\
\hline \multicolumn{7}{|c|}{ Peripheral: Structure } \\
\hline \multicolumn{7}{|c|}{ Muscle biopsy } \\
\hline Bierbrauer et al. (40) & & 5 & & & & \\
\hline Derde et al. (41) & & 15 & & & & \\
\hline Fredriksson et al. (35) & & & & & $\checkmark$ & \\
\hline Hooijman et al. (51) & & & & & $\checkmark$ & \\
\hline Klaude et al. (34) & & & & & $\checkmark$ & \\
\hline Llano-diez et al. (42) & & & & For seven to 11 days & & \\
\hline Puthucheary et al. (45) & & $1 ; 3 ; 7 ; 10$ & & & & \\
\hline Puthucheary et al. (52) & & $1 ; 7$ & & & & \\
\hline Puthucheary et al. (62) & & $1 ; 7$ & & & & \\
\hline Weber-castens et al. (47) & & 5 & & & & \\
\hline Wollersheim et al. (49) & & $5 ; 13$ & & & & \\
\hline \multicolumn{7}{|c|}{ Peripheral: Function } \\
\hline \multicolumn{7}{|c|}{ MRC-SS } \\
\hline Baldwin and Bersten (23) & & & & & $\checkmark$ & \\
\hline Bierbrauer et al. (40) & & 13 & & & & \\
\hline De Jonghe et al. (24) & & & & Day of awakening & & \\
\hline Weber-Carstens et al. (37) & & & & & $\checkmark$ & \\
\hline Wollersheim et al. (49) & & 13 & & & & \\
\hline \multicolumn{7}{|c|}{ Dynamometry } \\
\hline Baldwin and Bersten (23) & & & & & $\checkmark$ & \\
\hline \multicolumn{7}{|c|}{ Muscle torque via nerve stimulation } \\
\hline Ginz et al. (33) & & & & $\begin{array}{c}\text { After one week of intubation } \\
\text { and immobilisation }\end{array}$ & & \\
\hline Ginz et al. (36) & & & & $\begin{array}{l}\text { After two week of intubation } \\
\text { and immobilisation }\end{array}$ & & \\
\hline \multicolumn{7}{|c|}{ Electrophysiological measurements } \\
\hline Bierbrauer et al. (40) & & 7 & & & & \\
\hline Llano-diez et al. (42) & & & & For seven to 11 days & & \\
\hline Weber-Carstens et al. (37) & & & & $\begin{array}{c}\text { Within seven days of ICU } \\
\text { admission and thereafter on a } \\
\text { three-day interval }\end{array}$ & & \\
\hline Weber-Carstens et al. (47) & & & & $\begin{array}{l}\text { On admission and thereafter } \\
\text { on } 12 \text { consecutive days }\end{array}$ & & \\
\hline \multicolumn{7}{|c|}{ Postural: Structure } \\
\hline \multicolumn{7}{|c|}{ CT Scan } \\
\hline Sheean et al. (48) & & $7 ; 10 ; 14$ & & & & \\
\hline \multicolumn{7}{|c|}{ Muscle biopsy } \\
\hline Derde et al. (41) & & $10 ; 15$ & & & & \\
\hline
\end{tabular}

KEY: $\checkmark$-Yes; MIP - Maximal Inspiratory Pressure; MEP - Maximal Expiratory Pressure; MRC-SS - Medical Research Council Sum Score; CT - Computed Tomography 


\subsubsection{Testing procedure of the measurement tools for muscle structure and function}

The common muscles ultrasonography was conducted on were the right hemi-diaphragm, the vastus lateralis and the rectus femoris muscle. There were no studies that reported performing ultrasonography on postural muscles. The positioning of the patients, as well as the technique regarding probe placement was not standardised for the peripheral muscles, with different methods used amongst the studies. Computed Tomography (CT) scans were used to evaluate diaphragm thickness (Tdi) and cross-sectional area (CSA) of the postural muscles in the respective studies. $(56,48)$ Both studies reported on the procedure that was used.

The diaphragm and the vastus lateralis muscle were the two common muscles from which muscle biopsies were obtained. In the peripheral muscle group, no muscle biopsies were obtained from muscles of the upper limb. There was only one study that conducted a muscle biopsy on a postural muscle. (41) The procedure used to obtain muscle biopsies from the respiratory muscles were reported in all four studies. $(35,51,39,59)$ In the peripheral and postural muscle group, only four studies reported on the procedure used to obtain muscle biopsies, $(35,51,42,45)$ but eight studies reported on the method used to store the muscle biopsy after it was obtained. A common storage method reported amongst the studies for each respective muscle group entailed freezing the muscle biopsy in liquid nitrogen and storing it at $-80^{\circ}$ Celsius until analysis. $(34,35,39,41,42,45,47,51,52,59)$

Seven studies reported measuring diaphragmatic strength by means of bilateral anterior magnetic stimulation of the phrenic nerve (Table 2.2). A common placement of the magnetic coils used in majority of the studies was posterior to the sternocleidomastoid muscle at the level of the cricoid cartilage with the exception of two studies which reported a different location. $(32,46)$ Three studies measured endotracheal pressure (TwPett) and all three studies reported using the same procedure. $(32,39,44)$ Maximal inspiratory pressure (MIP) was measured in two studies, $(23,50)$ whereas both MIP and maximal expiratory pressure 
(MEP) were measured in the study conducted by De Jonghe et al. (24). Two studies used the same device and procedure to measure MIP. $(23,50)$ De Jonghe et al. $(24)$ used a different method whereby both MIP and MEP were measured via a tracheal tubing during a short disconnection from the ventilator.

Five studies reported using the MRC-SS to assess peripheral muscle strength (Table 2.2). In four studies, participants had to be alert according to the Richmond Agitation-Sedation Scale (RASS) and respond to the Standardised Five Questions (S5Q) which were: "open and close your eyes", "look at me", "open your mouth and put out your tongue", "nod your head" and "raise your eyebrows after I have counted to five." $(23,24,41,49)$ In contrast, one study did not specify using a criteria before conducting the test. (37) None of the studies reported the position participants were placed in to perform the test. Baldwin and Bersten (23) was the only study that reported using dynamometry to measure grip strength and strength of the elbow flexors and knee extensors. The Jamar Hydraulic Hand Dynamometer and the Lafayette Manual Muscle Test System were the two devices that were used. Patients were positioned in a modified recumbent position for testing of both outcomes. No other details were reported regarding how the test was performed and how many measurements were taken for each limb tested.

Ginz et al. $(33,36)$ were the two studies that quantified muscle force of the ankle dorsiflexors using nerve stimulation. Both studies reported using the same procedure. Electrophysiological compound muscle action potentials after direct muscle stimulation were performed in four studies (Table 2.2). This test was mainly performed on muscles of the lower limb with the exception of one study that conducted the test on muscles from both the upper limb and lower limb. (47) Only one article reported the placement of the electrodes as well as the stimulation values. (42) The methodology for each measurement tool can be found in Addendum E. 


\subsubsection{Changes in muscle structure and function reported in critically ill patients}

All studies that reported on Tdi obtained different results. Schepens et al. (53) was the only study that found a decline in Tdi to have reported the percentages (Table 2.4). Baldwin and Bersten (23) was the only study that reported a non-significant unchanged Tdi. Goligher et al. $(3,60)$ reported that the Tdi significantly increased, decreased and remained unchanged within the first week of both controlled and partially assisted mechanical ventilation. The diaphragm thickening fraction (DTF) significantly decreased in all three studies (Table 2.4). Umbrello et al. (54) was the only study that measured diaphragm excursion and reported that the diaphragm excursion remained unaltered and was not significant.

Peripheral muscle thickness and CSA significantly decreased in majority $(75 \%)$ of the studies. $(23,42,45,57,61)$ However, Turton et al. (57) reported that the muscle thickness of the medial head of the gastrocnemius and the elbow flexor compartment significantly remained unchanged on both day five and day 10 when compared to day one. In contrast, the same study found a significant loss of muscle thickness of the vastus lateralis muscle over both day five and day 10. (57) Puthucheary et al. (45) was the only study to report a $10.3 \%$ decline in the rectus femoris CSA between day one and day seven and continued to decrease up to day 10 , but did not provide the percentage rate per day by which it declined. Different results were obtained between the studies that measured echogenicity. Puthucheary et al. (52) was the only study that reported that the rectus femoris muscle echogenicity remained unchanged between day one and day 10 and was not significant (Table 2.4).

Both the Type I and Type II muscle fibres of the diaphragm muscle significantly decreased in the respective studies. $(51,59)$ However, both studies did not specify whether the reduction was observed in Type Ila or Type IIb muscle fibres of the fast twitch muscle group. Both Hooijman et al. (51) and Jaber et al. (39) reported an increase in the expression of the ubiquitin proteasomes in the diaphragm muscle, although Jaber et al. (39) found no change 
in either the Type I or Type II muscle fibres. However, the expression of the ubiquitin proteosome significantly increased in the study by Hooijman et al. (51), and not in the study by Jaber et al (39). Fredriksson et al. (35) looked at the enzymatic activity as well as the phosphate and lactate concentration of the intercostal muscles. The results by Fredriksson et al. (35) are reported in Table 2.4 and were not significant.

Reports on the messenger ribonucleic acid (mRNA) levels and changes in the Type I and Type II muscle fibres of the peripheral muscles were significant but variable in the respective studies. $(40,41,42,45,49,51,52)$ Puthucheary et al. (45) was the only study that reported a non-significant decrease in Type I and Type II muscle fibres. The muscle protein content of the vastus lateralis and tibialis anterior muscles significantly decreased in all four studies. $(40,41,42,49)$ A summarised tabulation of the reported measures can be perused in Table 2.4 .

Lee et al. (56) reported that the thickness of the left and right side of the diaphragm significantly decreased on both the first and second CT scan with a $10 \%$ reduction in total (54). Sheean et al. (48) found a decline in the CSA of the postural muscles in patients with respiratory failure on day seven, 10 and 14 , but was not significant.

Majority of the studies measuring respiratory muscle strength reported a significant decrease in the measures of MIP, trans-diaphragmatic pressure and endotracheal pressure. The exception was Bisset (50), whom reported a wide variability in MIP scores ranging between 6 $\mathrm{cmH}_{2} \mathrm{O}$ to $87 \mathrm{cmH}_{2} \mathrm{O}$, with one patient scoring $86 \%$ of their predicted MIP. Furthermore, Baldwin and Bersten (23) reported a non-significant decrease in MEP. A significant decrease in upper limb, lower limb and grip strength was reported in studies using the MRCSS and dynamometry to assess peripheral muscle strength in critically ill patients. The two studies that measured muscle torque via nerve stimulation found a significant decrease in peak torque, peak torque development and contraction time. $(33,36)$ Although the two 
studies measured the same outcomes, discrepancies were found between the two studies when the peak torque decay significantly decreased in the one study (33) and significantly remained unchanged in the other study. (36) A significant decrease in electrophysiological measurements was obtained in three of the four studies with the exception of Llano-diez et al. (42) which reported no change, but was not significant. A summary of the results for the measures of muscle function are listed in Table 2.5. 
Table 2.4: Summary of the results for measurements of muscle structure

\begin{tabular}{|c|c|c|c|c|c|c|c|}
\hline Author(s) & Inc & Dec & $\mathrm{NC}$ & Percentage & Significant & Time points & Other \\
\hline \multicolumn{8}{|c|}{ Respiratory } \\
\hline \multicolumn{8}{|c|}{ Ultrasonography } \\
\hline \multicolumn{8}{|c|}{ Diaphragm thickness (Tdi) } \\
\hline $\begin{array}{c}\text { Baldwin and Bersten } \\
(23)\end{array}$ & & & $\checkmark$ & & & & \\
\hline Cartwright et al. (43) & $\checkmark$ & $\checkmark$ & & & $\checkmark$ & $\begin{array}{l}\uparrow: \text { Baseline to Day } 14 \\
\downarrow: 2,4-\& 6 \text {-months } \\
\text { Post-hospital } \\
\text { discharge }\end{array}$ & \\
\hline Goligher et al. (3) & $\checkmark$ & $\checkmark$ & $\checkmark$ & $\begin{array}{l}\uparrow:>10 \% \text { ( } 12 \% \text { patients) } \\
\downarrow:>10 \% \text { ( } 44 \% \text { of patients) } \\
\text { Unchanged ( } 44 \% \text { of } \\
\text { patients) }\end{array}$ & $\checkmark$ & $\begin{array}{l}\text { Over the first week of } \\
\text { MV }\end{array}$ & \\
\hline Goligher et al. (60) & $\checkmark$ & $\checkmark$ & $\checkmark$ & $\begin{array}{l}\uparrow=>10 \%(25 \% \text { of } \\
\text { patients) } \\
\downarrow=>10 \%(41 \% \text { patients }) \\
\text { Unchanged ( } 34 \% \text { of } \\
\text { patients) }\end{array}$ & $\checkmark$ & $\uparrow:$ First week of MV & \\
\hline Schepens et al. (53) & & $\checkmark$ & & $\begin{array}{l}24 \text { hours of MV: } 9 \% \\
48 \text { hours of } M V: 20 \% \\
72 \text { hours of } M V: 26 \% \\
\text { Average: } 10.9 \%\end{array}$ & $\checkmark$ & & \\
\hline Umbrello et al. (54) & & $\checkmark$ & & & $\checkmark$ & & \\
\hline Zambon et al. (58) & & $\checkmark$ & & & $\checkmark$ & & \\
\hline \multicolumn{8}{|c|}{ Diaphragm Thickening Fraction (DTF) } \\
\hline Goligher et al. (60) & $\checkmark$ & $\checkmark$ & & & $\checkmark$ & After 7 days of $\mathrm{MV}$ & \\
\hline Umbrello et al. (54) & & $\checkmark$ & & & $\checkmark$ & & \\
\hline Zambon et al. (58) & & $\checkmark$ & & & $\checkmark$ & & \\
\hline \multicolumn{8}{|c|}{ Diaphragm Excursion } \\
\hline Umbrello et al. (54) & & & $\checkmark$ & & & & \\
\hline \multicolumn{8}{|c|}{ CT Scan } \\
\hline \multicolumn{8}{|c|}{ Diaphragm thickness (Tdi) } \\
\hline Lee et al. (56) & & $\checkmark$ & & $10 \%$ & $\checkmark$ & Second CT scan & \\
\hline \multicolumn{8}{|c|}{$\begin{array}{ll} & \text { Muscle Biopsy } \\
\end{array}$} \\
\hline Fredriksson et al. (35) & \multicolumn{4}{|c|}{$\begin{array}{l}\downarrow \text { Mitochondrial enzyme activities } \\
\downarrow \text { ATP and creatine phosphate concentration } \\
\uparrow \text { Lactate concentration }\end{array}$} & & & \\
\hline Jaber et al. (39) & \multicolumn{4}{|c|}{$\begin{array}{l}\text { Muscle fibres: Slow-twitch and fast-twitch fibres } \\
\text { remained unchanged } \\
\text { Ubiquitin proteasomes: } \uparrow \text { Expression of the } \\
\text { ubiquinated proteins }\end{array}$} & & Not specified & \\
\hline Hooijman et al. (51) & \multicolumn{4}{|c|}{$\begin{array}{l}\text { Muscle fibres: } \downarrow \text { Both the slow-twitch and fast- } \\
\text { twitch fibres } \\
\text { Ubiquitin proteasomes: } \uparrow \text { Expression of the } \\
\text { ubiquinated proteins }\end{array}$} & $\checkmark$ & & \\
\hline Van den Berg et al. (59) & \multicolumn{4}{|c|}{$\begin{array}{l}\text { Muscle fibres: } \downarrow \text { Both the slow-twitch and fast- } \\
\text { twitch fibres } \\
\text { Muscle protein: } \downarrow \text { Pro-fusion proteins }\end{array}$} & $\checkmark$ & Not specified & \\
\hline \multicolumn{8}{|c|}{$\begin{array}{ll} & \text { Peripheral } \\
\end{array}$} \\
\hline \multicolumn{8}{|c|}{ Ultrasonography } \\
\hline \multicolumn{8}{|c|}{ Muscle Thickness } \\
\hline $\begin{array}{l}\text { Baldwin and Bersten } \\
(23)\end{array}$ & & $\checkmark$ & & & $\checkmark$ & & \\
\hline Cartwright et al. (43) & $\checkmark$ & & & & $\checkmark$ & & \\
\hline $\begin{array}{c}\text { Hernandez-Scorro et al. } \\
(61)\end{array}$ & & $\checkmark$ & & & $\checkmark$ & & \\
\hline Turton et al. (57) & & $\checkmark$ & $\checkmark$ & & $\checkmark$ & & \\
\hline \multicolumn{8}{|c|}{ Cross-sectional Area (CSA) } \\
\hline $\begin{array}{l}\text { Hernandez-Scorro et al. } \\
(61)\end{array}$ & & $\checkmark$ & & & $\checkmark$ & & \\
\hline Llano-diez et al. (42) & & $\checkmark$ & & & $\checkmark$ & For 7 to 11 days & \\
\hline Puthucheary et al. (45) & & $\checkmark$ & & $10.3 \%$ & $\checkmark$ & $\begin{array}{l}\text { Day } 1 \text { - Day } 7, \\
\text { Day } 10\end{array}$ & \\
\hline Puthucheary et al. (52) & & & $\checkmark$ & & $\checkmark$ & Day 1 - Day 10 & \\
\hline
\end{tabular}


Table 2.4: Continued

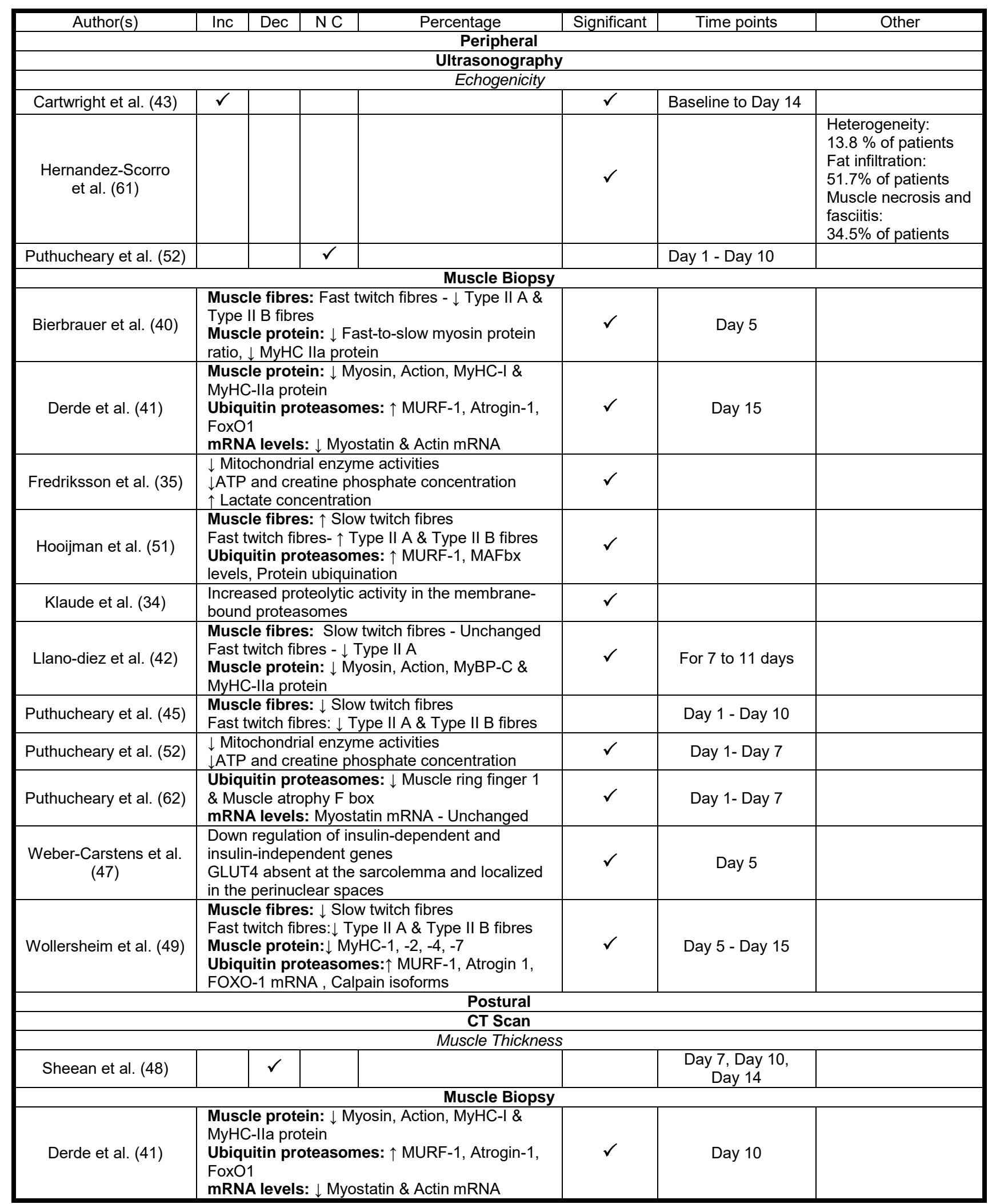

KEY: $\checkmark$-Yes; $\uparrow$ - Increase; $\downarrow$ - Decrease; Inc - Increase, Dec - Decrease; N C - No Change; MV - Mechanical Ventilation; MyHC - Myosin Heavy Chain; MURF - Muscle Ring-Finger protein; MAFbx - Muscle Atrophy F-box gene; MyBP - Myosin-Binding protein; mRNA - messenger Ribonucleic Acid 
Table 2.5: Summary of the results for measurements of muscle function

\begin{tabular}{|c|c|c|c|c|c|c|}
\hline Author(s) & Inc & Dec & NC & Significant & Time points & Other \\
\hline \multicolumn{7}{|c|}{ Respiratory } \\
\hline \multicolumn{7}{|c|}{ MIP } \\
\hline $\begin{array}{c}\text { Baldwin and Bersten } \\
(23)\end{array}$ & & $\checkmark$ & & $\checkmark$ & & \\
\hline Bissett (50) & & & & & After 48 hours of MV & Wide variability \\
\hline De Jonghe et al. (24) & & $\checkmark$ & & $\checkmark$ & $\begin{array}{c}\text { Day of awakening- Not } \\
\text { specified }\end{array}$ & \\
\hline \multicolumn{7}{|c|}{ MEP } \\
\hline De Jonghe et al. (24) & & $\checkmark$ & & & & \\
\hline \multicolumn{7}{|c|}{ Bilateral Phrenic Nerve Stimulation: Trans-diaphragmatic Pressure } \\
\hline Buscher et al. (32) & & $\checkmark$ & & $\checkmark$ & Not specified & \\
\hline Hermans et al. (38) & & $\checkmark$ & & & Not specified & \\
\hline $\begin{array}{c}\text { Supinski and Callahan } \\
(46)\end{array}$ & & $\checkmark$ & & $\checkmark$ & & \\
\hline Umbrello et al. (54) & & $\checkmark$ & & $\checkmark$ & & \\
\hline \multicolumn{7}{|c|}{ Bilateral Phrenic Nerve Stimulation: Endotracheal Pressure } \\
\hline Buscher et al. (32) & & $\checkmark$ & & $\checkmark$ & Not specified & \\
\hline Demoule et al. (44) & $\checkmark$ & $\checkmark$ & & & & $\begin{array}{l}\text { Day 1: } \downarrow \text { (64 \% patients) } \\
\text { Between Day } 1 \text { and Day 3: } \\
\downarrow>1 \mathrm{~cm} \text { (38\% patients), } \\
\uparrow>1 \mathrm{~cm} \text { (31\% patients) } \\
\text { Day 3: } \downarrow \text { (63\% patients) }\end{array}$ \\
\hline Demoule et al. (55) & & $\checkmark$ & & $\checkmark$ & Initiation of MV to Day 7 & \\
\hline Jaber et al. (39) & & $\checkmark$ & & $\checkmark$ & Day 5-6 of MV & \\
\hline \multicolumn{7}{|c|}{ Peripheral } \\
\hline \multicolumn{7}{|c|}{ Medical Research Council Sum Score (MRC-SS) } \\
\hline $\begin{array}{l}\text { Baldwin and Bersten } \\
(23)\end{array}$ & & $\checkmark$ & & $\checkmark$ & & \\
\hline Bierbrauer et al. (40) & & $\checkmark$ & & $\checkmark$ & Day 13 & \\
\hline De Jonghe et al. (24) & & $\checkmark$ & & $\checkmark$ & $\begin{array}{c}\text { Day of awakening- Not } \\
\text { specified }\end{array}$ & \\
\hline $\begin{array}{c}\text { Weber-Carstens et al. } \\
(37)\end{array}$ & & $\checkmark$ & & $\checkmark$ & & ICUAW: $57 \%$ of patients \\
\hline Wollersheim et al. (49) & & $\checkmark$ & & & Day 13 & \\
\hline \multicolumn{7}{|c|}{ Dynamometry } \\
\hline $\begin{array}{l}\text { Baldwin and Bersten } \\
(23)\end{array}$ & & $\checkmark$ & & $\checkmark$ & & \\
\hline \multicolumn{7}{|c|}{ Muscle Torque via Nerve Stimulation } \\
\hline Ginz et al. (33) & HRT & $\begin{array}{l}\text { PT, PTD, } \\
\text { PTd, CT }\end{array}$ & & $\checkmark$ & Not specified & \\
\hline Ginz et al. (36) & & $\begin{array}{c}\text { PT, PTD, } \\
\text { CT }\end{array}$ & PTd & $\checkmark$ & Not specified & \\
\hline \multicolumn{7}{|c|}{ Electrophysiological Measurements } \\
\hline Bierbrauer et al. (40) & & $\checkmark$ & & $\checkmark$ & Day 7 & \\
\hline Llano-diez et al. (42) & & & $\checkmark$ & & For 7 to 11 Days & \\
\hline $\begin{array}{c}\text { Weber-Carstens et al. } \\
\text { (37) }\end{array}$ & & $\checkmark$ & & $\checkmark$ & Day 5 after ICU admission & \\
\hline $\begin{array}{c}\text { Weber-Carstens et al. } \\
(47)\end{array}$ & & $\checkmark$ & & $\checkmark$ & Day 12 & \\
\hline
\end{tabular}

KEY: $\checkmark$-Yes; Inc - Increase; Dec - Decrease; N C - No Change; MV- Mechanical Ventilation; MIP - Maximal Inspiratory Pressure; MEP - Maximal Expiratory Pressure; HRT - Half-Relaxation Time; PT - Peak Torque; PTD - Peak Torque Development; PTd - Peak Torque decay; CT - Contraction Time 


\subsubsection{Factors associated with change in muscle structure and function}

Table 2.6 represents the factors that were reported across the 22 studies, with sepsis $(n=10)$, neuromuscular-blocking agents (NMBA) $(n=5)$, and mechanical ventilation $(n=5)$ being the most common factors associated with change in muscle structure and function. 
Table 2.6: Factors associated with change in muscle structure and function

\begin{tabular}{|c|c|c|c|c|c|c|c|c|c|c|c|c|c|}
\hline Authors & Year & Sepsis & $\begin{array}{l}\text { Septic } \\
\text { shock }\end{array}$ & $\begin{array}{l}\text { Severity } \\
\text { of illness }\end{array}$ & $\begin{array}{l}\text { Mechanical } \\
\text { ventilation }\end{array}$ & Corticosteroids & NMBA & Propofol & $\begin{array}{l}\text { Glycaemic } \\
\text { control }\end{array}$ & $\begin{array}{l}\text { Electrolyte } \\
\text { disturbance }\end{array}$ & Immobilisation & Other & $\begin{array}{c}\text { Not } \\
\text { Reported }\end{array}$ \\
\hline Buscher et al. (32) & 2005 & & & & & & & & & & & $\begin{array}{c}\text { Positive end- } \\
\text { expiratory pressure }\end{array}$ & \\
\hline Ginz et al. (33) & 2005 & & & & & & & $\checkmark$ & & $\checkmark$ & $\checkmark$ & & \\
\hline Klaude et al. (34) & 2005 & & & & & & & & & & & & $\checkmark$ \\
\hline $\begin{array}{c}\text { Fredriksson et al. } \\
(35)\end{array}$ & 2006 & & & & & & & & & & & & $\checkmark$ \\
\hline $\begin{array}{c}\text { De Jonghe et al. } \\
(24)\end{array}$ & 2007 & & $\checkmark$ & & & & & & & & & & \\
\hline Ginz et al. (36) & 2008 & & & & & & & $\checkmark$ & & & & & \\
\hline $\begin{array}{l}\text { Weber-Carstens et } \\
\text { al. (37) }\end{array}$ & 2009 & & & & & & $\checkmark$ & & & & & Hydrocortisone & \\
\hline $\begin{array}{l}\text { Hermans et al. } \\
\text { (38) }\end{array}$ & 2010 & $\checkmark$ & & & & $\checkmark$ & & & $\checkmark$ & & & & \\
\hline Jaber et al. (39) & 2011 & & & & & & & & & & & & $\checkmark$ \\
\hline $\begin{array}{c}\text { Bierbrauer et al. } \\
(40)\end{array}$ & 2012 & & & & & & & & & & & & $\checkmark$ \\
\hline Derde et al. (41) & 2012 & & & & & $\checkmark$ & $\checkmark$ & & & & & & \\
\hline $\begin{array}{l}\text { Llano-Diez et al. } \\
\text { (42) }\end{array}$ & 2012 & $\checkmark$ & & & & $\checkmark$ & $\checkmark$ & & & & & $\begin{array}{c}\text { Hormone } \\
\text { administration }\end{array}$ & \\
\hline $\begin{array}{c}\text { Cartwright et al. } \\
(43)\end{array}$ & 2013 & & & & & & & & & & & Fluid resuscitation & \\
\hline $\begin{array}{c}\text { Demoule et al. } \\
(44)\end{array}$ & 2013 & $\checkmark$ & & $\checkmark$ & & & & & & & & & \\
\hline $\begin{array}{c}\text { Puthucheary et al. } \\
(45)\end{array}$ & 2013 & & & & & & & & & & $\checkmark$ & & \\
\hline $\begin{array}{l}\text { Supinski and } \\
\text { Callahan (46) }\end{array}$ & 2013 & $\checkmark$ & & & & & & & & & & & \\
\hline $\begin{array}{l}\text { Weber-Carstens et } \\
\text { al. (47) }\end{array}$ & 2013 & $\checkmark$ & & $\checkmark$ & & & & & & & & & \\
\hline $\begin{array}{l}\text { Baldwin and } \\
\text { Bersten (23) }\end{array}$ & 2014 & $\checkmark$ & & $\checkmark$ & & & & & & & & $\begin{array}{l}\text { Markers of Systemic } \\
\text { Inflammation (IL-6) }\end{array}$ & \\
\hline Sheean et al. (48) & 2014 & & & & & & & & & & & & $\checkmark$ \\
\hline $\begin{array}{c}\text { Wollersheim et al. } \\
(49)\end{array}$ & 2014 & $\checkmark$ & & & & & & & & & & $\begin{array}{c}\text { Systemic } \\
\text { inflammation }\end{array}$ & \\
\hline
\end{tabular}


Table 2.6: Continued

\begin{tabular}{|c|c|c|c|c|c|c|c|c|c|c|c|c|c|}
\hline Authors & Year & Sepsis & $\begin{array}{l}\text { Septic } \\
\text { shock }\end{array}$ & $\begin{array}{l}\text { Severity } \\
\text { of illness }\end{array}$ & $\begin{array}{l}\text { Mechanical } \\
\text { ventilation }\end{array}$ & Corticosteroids & NMBA & Propofol & $\begin{array}{l}\text { Glycaemic } \\
\text { control }\end{array}$ & $\begin{array}{l}\text { Electrolyte } \\
\text { disturbance }\end{array}$ & Immobilisation & Other & $\begin{array}{c}\text { Not } \\
\text { Reported }\end{array}$ \\
\hline Bissett (50) & 2015 & & & & & & & & & & & & $\checkmark$ \\
\hline Goligher et al. (3) & 2015 & & & $\checkmark$ & $\checkmark$ & & & & & & & & \\
\hline $\begin{array}{c}\text { Hooijman et al. } \\
(51)\end{array}$ & 2015 & & & & & & & & & & & & $\checkmark$ \\
\hline $\begin{array}{l}\text { Puthucheary et al. } \\
\text { (52) }\end{array}$ & 2015 & & & & & & & & & & & & $\checkmark$ \\
\hline $\begin{array}{c}\text { Schepens et al. } \\
(53)\end{array}$ & 2015 & & & & & & & & & & & $\begin{array}{c}\text { Time spent on the } \\
\text { ventilator }\end{array}$ & \\
\hline $\begin{array}{c}\text { Umbrello et al. } \\
(54)\end{array}$ & 2015 & & & & & & & & & & & & $\checkmark$ \\
\hline $\begin{array}{c}\text { Demoule et al. } \\
(55)\end{array}$ & 2016 & $\checkmark$ & & & $\checkmark$ & & & & & & & & \\
\hline Lee et al. (56) & 2016 & $\checkmark$ & & & $\checkmark$ & & & & & $\checkmark$ & & $\begin{array}{l}\text { Malnutrition, } \\
\text { Reduced perfusion } \\
\text { to the diaphragm }\end{array}$ & \\
\hline Turton et al. (57) & 2016 & & & & & & & & & & & & $\checkmark$ \\
\hline Zambon et al. (58) & 2016 & & & & $\checkmark$ & & $\checkmark$ & & & & & Sedation & \\
\hline $\begin{array}{l}\text { Van den Berg et } \\
\text { al. (59) }\end{array}$ & 2017 & & & & & & & & & & & & $\checkmark$ \\
\hline Goligher et al. (60) & 2018 & & & & $\checkmark$ & & & & & & & $\begin{array}{l}\text { Prolong ICU } \\
\text { Admission }\end{array}$ & \\
\hline $\begin{array}{l}\text { Hernandez-Scorro } \\
\text { et al. (61) }\end{array}$ & 2018 & $\checkmark$ & & & & $\checkmark$ & $\checkmark$ & & & & & & \\
\hline $\begin{array}{l}\text { Puthucheary et al. } \\
\text { (61) }\end{array}$ & 2018 & & & & & & & & & & & & $\checkmark$ \\
\hline
\end{tabular}

KEY: $\checkmark$ - Yes; NMBA - Neuromuscular Blocking Agents 


\subsection{Discussion}

A scoping review was undertaken to map the current literature reporting on muscle structure and function in critically ill patients. The map indicates the following: 1) a wide range of populations have been investigated; 2) data available was predominantly from developed countries; 3 ) assessments were focused on either muscle structure or muscle function, and rarely both; 4) the postural muscles were the least investigated; 5) majority of assessments took place early in the ICU stay; and 6) neither the muscle structure assessment nor the muscle function assessments were standardised.

In this review, research regarding muscle structure and function was not conducted in developing countries. Therefore, it is unknown what the results are regarding muscle structure and function in a patient population from a developing country. Furthermore, the difference between a developed and a developing country could reflect that of a different population group. A developed country would have an older patient population with comorbidities whereas, a developing country would have a younger patient population where majority would be malnourished due to poverty. Majority of the studies were published between 2013 and 2015. The advancement in critical care medicine has led to an increase in the number of critically ill survivors, thus decreasing the mortality rate, but increasing morbidity. (63) These improvements in survival after critical illness has led to ICU survivors experiencing a persistent reduction in physical function, impairments in mental health, cognitive dysfunction and a decrease in HRQoL. First recognised in 2012, this constellation of problems is called post-intensive care syndrome (PICS) and could be the reason as to why research has been focused on assessing the trajectory of these survivors thereafter.

Measurements were conducted at different time intervals during the patient ICU stay, indicating that no specific protocol exist as to when measurements should be taken. There were only a few follow-ups post-ICU discharge that observed the trajectory of critically ill 
survivors. Therefore, though it is known what happens to the structure and function of muscles during ICU stay, it is unknown whether these results post-ICU and post-hospital discharge remains unchanged, continues to deteriorate or improves. Hence, more research is required on the frequency of measurements post-ICU and post-hospital discharge. This will assist in understanding the morbidities critically ill survivors presents with and in designing interventions to address these morbidities.

Ultrasonography and muscle biopsies were the two common measurement tools used to study peripheral muscle structure. However, the methodology of these measurement tools varied. Studies reported using different probe placements for imaging common muscles such as the vastus lateralis and rectus femoris muscles as well as different procedures to obtain muscle biopsies. Thus, further research is required to standardise the methodology of these two measurement tools. It is interesting to note that the MRC-SS was used to quantify peripheral muscle strength in critically ill patients even though it has a low ceiling effect and requires extensive training and quality assurance of a clinician to ensure high intra-rater reliability. (64) Although complex and invasive in nature, trans-diaphragmatic pressure via bilateral anterior magnetic stimulation of the phrenic nerve was the tool that was routinely used to measure diaphragmatic strength as compared to non-invasive tools such as MIP and MEP.

There was a lack of research conducted on postural muscles in critically ill patients. Current research has shown that when postural muscles are exposed to microgravity such as bed rest and immobilisation for at least seven days, it causes atrophy of Type I and Type II muscle fibres resulting in a lower fatigue resistance capacity. (65) Furthermore, atrophy of type II muscle fibres of the rectus abdominus muscle could be the reason as to why critically ill patients who are mechanically ventilated experience difficulty expectorating secretions as well as have impairments in physical function. Therefore, more research is required on postural muscles. 
There was no consistency in the results for muscle structure, even though generally, results showed a decrease in Tdi and DTF of the respiratory muscles, a decrease in the muscle thickness and CSA of the peripheral muscles as well as reduction in the Type I and Type II muscle fibres and muscle protein content of both the respiratory and peripheral muscles. Due to this variation, the results for muscle structure should be interpreted with caution. This variability in results could be due to: i) the presence of tissue oedema; ii) that non-weight bearing muscles such as the respiratory muscles and muscles of the upper limb are less susceptible to muscle wasting as compared to weight bearing muscles which have a load placed on them through the action of daily activities; iii) that the validity of the measurements tools is unknown; and iv) that the methodology for the measurements of muscle structure and function were not standardised.

A decrease in the measurements of respiratory and peripheral muscle strength was reported in majority of the studies. However, these results should be interpreted with caution as the measures for muscle function were not standardised. There were studies that conducted measurements on both muscle structure and function, however failed to draw an association between the two. Therefore, it is unknown how a loss of muscle mass would influence muscle function. Furthermore, no correlation was made between muscle structure and function with physical function and thus, literature supporting that poor physical function in critically ill survivors is a result of muscle structure and function could be based on postulations.

\subsection{Limitation and recommendation}

The aim of this scoping review was to map the current understanding of muscle structure and function in critically ill patients. Recommendations for future research are to investigate the correlation between muscle structure and muscle function as well as the relationship between muscle structure and function with physical function in critically ill patients. The use 
of non-invasive measurement tools such as MIP, MEP and dynamometry in the ICU should also be considered in the future.

This scoping review has some limitations. The selection of the databases for the 34 studies included in this review only produced studies from developed countries. Therefore, the results of muscle structure and function in this review of critically ill patients reflects that of a developed population. Research in this scoping review did not include the entire musculoskeletal system in its understanding of muscle structure and function in critically ill patients. Lastly, only studies published in English were included in this scoping review.

\subsection{Conclusion}

Though research has been focussed on muscle structure and function, it is evident that the current literature is inadequate in understanding muscle structure and function in critically ill patients. 


\section{Chapter 3 : Methodology of the Primary Research Study}

The methodology for the primary research study and the description of each measurement tool used to measure muscle structure, muscle function and physical function will be presented in this chapter.

\subsection{Research question}

What is the relationship between muscle structure, muscle function and physical function in critically ill patients at Intensive Care Unit (ICU) discharge and hospital discharge?

\subsection{Research aim}

The aim of this study was to determine the correlation between muscle structure, muscle function and physical function in critically ill patients at ICU discharge and hospital discharge in a South African population setting.

\subsection{Research objectives}

\subsubsection{Respiratory muscle structure and function in a critically ill population}

- To describe the change in muscle thickness of the diaphragm muscle through ultrasonography from ICU admission for three consecutive days.

- To determine and describe respiratory muscle strength at ICU discharge and at hospital discharge.

- To determine and describe respiratory muscle endurance at hospital discharge.

\subsubsection{Peripheral muscle structure and function in a critically ill population}

- To describe the muscle thickness, cross-sectional area (CSA) and echogenicity of the quadriceps muscle through ultrasonography from ICU admission for three consecutive days. 
- To determine peripheral muscle strength via the Medical Research Council-Sum Score (MRC-SS) when the patient is awake during their ICU stay, at ICU discharge and at hospital discharge.

- To determine peripheral muscle strength through dynamometry at hospital discharge.

\subsubsection{Physical function and health-related quality of life (HRQoL) in a critically ill} population

- To determine and describe the physical function capabilities by completing the Chelsea Critical Care Physical Assessment (CPAx) tool when the patient is awake during their ICU stay and at ICU discharge.

- To determine and describe the physical function capabilities by completing the de Morton Mobility Index (DEMMI) tool at hospital discharge.

- To determine the functional exercise capacity by completing the Six-Minute Walk Test (6-MWT) at hospital discharge.

- To determine and describe the health-related quality of life (HRQoL) by completing the EQ-5D-5L questionnaire at hospital discharge.

\subsubsection{Associations between muscle structure, muscle function and physical function} in a critically ill population

\subsubsection{At ICU awakening}

- To determine the relationship between diaphragm thickness (Tdi) at end-expiration with the CPAx tool.

- To determine the relationship between the diaphragm thickening fraction (DTF) with the CPAx tool.

- To determine the relationship between quadriceps muscle thickness, CSA and echogenicity with the MRC-SS.

- To determine the relationship between quadriceps muscle thickness, CSA and echogenicity with the CPAx tool.

- To determine the relationship between the MRC-SS and the CPAx tool. 


\subsubsection{At ICU discharge}

- To determine the relationship between the MRC-SS and the CPAx tool.

- To determine the relationship between respiratory muscle strength and the CPAx tool.

\subsubsection{At hospital discharge}

- To determine the relationship between the MRC-SS with the DEMMI and the 6-MWT.

- To determine the relationship between dynamometry (isometric knee extension force and grip strength) with the DEMMI and the 6-MWT.

- To determine the relationship between respiratory muscle strength and endurance with the 6-MWT.

- $\quad$ To determine the relationship between HRQoL with the DEMMI and the 6-MWT.

- $\quad$ To determine the relationship between the DEMMI and the 6-MWT.

\subsubsection{Demographics and clinical characteristics}

- To describe the demographics (age, gender, employment status, social history) and clinical characteristics (comorbidities, severity of illness, admission diagnosis, length of stay, ventilation mode, setting and measures, medication, sedation) of the participants.

\subsection{Research context and setting}

This was a prospective observational cohort study conducted in the medical and surgical ICUs of a Tertiary Academic Hospital in South Africa from August 2019 to February 2020.

\subsection{Ethical consideration}

This study is part a larger study titled "Impact of critical illness on muscle structure, strength and physical capacity". Ethical approval was obtained from the Health Research and Ethical Committee at the Faculty of Health Sciences of Stellenbosch University (Ethics no: S16/09/173A) (Addendum F). Institutional permission was granted by the Chief Executive 
Officer of Tygerberg hospital (Addendum G). Informed consent in writing was obtained from the participants. Consent forms were available in English, Afrikaans and isiXhosa (Addendum $\mathrm{H}$ ). If the participant could not provide consent due to acute stages of critical illness (sedated/unconscious), deferred consent was applied by the primary investigator (PI) (in collaboration with the intensivists) until such time the participant was awake and thereafter requested for consent.

\subsection{Population sample}

All patients admitted to the medical and surgical ICUs were screened daily by the PI from the $1^{\text {st }}$ of August 2019 to the $28^{\text {th }}$ of February 2020.

Participants were included in the study if they were:

(i) Adults between the age of 18 and 65 years

(ii) Intubated and mechanically ventilated within 24 hours of ICU admission

(iii) On Non-Invasive Ventilation (NIV) for $>18$ hours a day

Participants were excluded from the study if they presented with:

(i) Acute or chronic neuromuscular disorders and/or diaphragm injury on admission

(ii) Spinal cord lesions

(iii) Neurological conditions such as Traumatic Brain Injury (TBI) and/or Cerebrovascular Accident (CVA)

(iv) Orthopaedic conditions such as pelvic fractures or lower limb fractures where weightbearing was contraindicated

(v) $\quad A B M I \geq 40 \mathrm{~kg} / \mathrm{m}^{2}$

(vi) Did not provide informed consent

\subsection{Sample size}

Based on clinical reasoning, it was hypothesised that 13 participants would obtain a strong correlation of $R=0.7$ between muscle structure, muscle function and physical function at 
ICU discharge and at hospital discharge. However, to prevent bias, all data collected on muscle structure, muscle function and physical function were used to determine correlations at ICU awakening, ICU discharge and hospital discharge. Moreover, at hospital discharge due to the loss of follow-up of participants, only 13 participants with full data sets were used to determine correlations between muscle structure, muscle function and physical function. The data provided in this study can be used for future significantly powered research studies.

\subsection{Research team}

The research team consisted of the PI, intensivists and the statistician. The duties of each candidate are listed below:

\subsubsection{Primary investigator $(\mathrm{PI})$}

- Screening participants admitted to the medical and surgical ICUs of a Tertiary Academic Hospital according to the inclusion and exclusion criteria in collaboration with the intensivists.

- Obtaining informed consent from the participants or next of kin.

- Applying or implementing deferred consent in collaboration with the intensivists.

- Allocation of a participant number after informed/deferred consent was obtained.

- Collection of baseline data from the medical file and charts for each participant.

- All measurements of muscle structure, muscle function and physical function in the ICU and the ward.

- Electronic capturing of data.

\subsubsection{Intensivists}

- Screening of participants for possible inclusion and exclusion together with the PI. 


\subsubsection{Statistician}

- Statistical analysis and interpretation of data after the completion of data collection together with the PI.

\subsection{Outcome measurement tools}

\subsubsection{Muscle structure and function}

\subsubsection{Ultrasonography of the diaphragm and quadriceps muscle}

Ultrasonography is a non-invasive tool and can be used to investigate diaphragm atrophy and dysfunction, predict extubation success or failure and quantify respiratory effort in mechanically ventilated patients. (66) The following outcomes of the diaphragm can be assessed using ultrasonography: Diaphragm thickness (Tdi), diaphragm thickening fraction (DTF) and diaphragm excursion.

Diaphragm thickness (Tdi) is used to determine diaphragmatic atrophy. Tdi is measured at either end-inspiration, end-expiration and/or both. $(3,53,58,67)$ During end-inspiration, the diaphragm is at its thickest, due to muscle contraction whereas during end-expiration, the diaphragm is at its thinnest as the muscle is relaxed and correlates to functional residual capacity (FRC). Diaphragm thickening fraction (DTF) relates to the function of the diaphragm and has been used in several studies as a predictor of extubation. $(67,68,69)$ DTF represents both the maximal and minimal Tdi during end-inspiration and end-expiration during a normal tidal breath (58) and is calculated as a percentage (\%) using the following formula: (67)

\section{(Thickness at end inspiration - Thickness at end-expiration)}

(Thickness at end-expiration)

$$
\times 100=\%
$$

Therefore, in this study Tdi and DTF was assessed using ultrasonography. 
Ultrasonography can also be used to evaluate quadriceps muscle in critically ill patients. The quadriceps are composed of four muscles namely, rectus femoris (RF), vastus intermedius $(\mathrm{VI})$, vastus lateralis $(\mathrm{VL})$, and vastus medialis $(\mathrm{VM})$, and are all made up of different fibre type compositions and have different functional roles. For example, RF is a fast twitch muscle made up of type II muscle fibres and is responsible for power and speed. The VI is a stabiliser muscle made up of type I muscle fibres and is responsible for dynamic balance. (26) The outcomes assessed using ultrasonography are muscle thickness, cross-sectional area (CSA) and echogenicity. Muscle echogenicity represents changes in the quality of the muscle. Normal muscle appears dark and dense (hypoechoic) on ultrasonography whereas a muscle appearing whiter or brighter (hyperechoic) may be representative of muscle necrosis and infiltration of fatty and connective tissue in place of muscle fibres. (26) Therefore, in this study quadriceps muscle thickness, CSA and echogenicity was assessed using ultrasonography.

\subsection{Reliability and validity}

Inter-rater and intra-rater reliability for ultrasonography of the diaphragm and quadriceps muscle have been demonstrated in several studies. A study conducted by Francis, Hoffer and Reynolds (70) reported a very good inter-rater reliability for measurements of Tdi and quadriceps muscle thickness and echogenicity $(I C C=0.95)$. Sarwal et al. $(71)$ reported a variation in ICC values ranging from 0.84 to 0.99 for inter-rater reliability for measurements of Tdi, and RF and VI muscle thickness and echogenicity. Baldwin et al. (72) reported a very good intra-rater reliability for measuring Tdi at end-expiration $(I C C=0.99)$ and for quadriceps muscle thickness $(I C C=1.0)$.

\subsubsection{Medical Research Council-Sum Score (MRC-SS)}

The Medical Research Council-Sum Score (MRC-SS) is currently the recommended method for assessing peripheral muscle strength and diagnosing intensive care unit-acquired weakness (ICU-AW) in critically ill patients in an ICU setting. Physical examination entails 
assessing three muscle groups of the upper limbs and three muscle groups of the lower limbs. It has a grading scale ranging from zero (no muscle contraction) to five (full muscle strength) with a composite score out of 60 . (11) A score of less than 48 out of 60 on the MRC-SS is defined as ICU-AW. $(11,73)$

\subsection{Reliability and validity}

The Medical Research Council-Sum Score (MRC-SS) is a reliable method for comprehensively assessing muscle strength in critically ill patients. Three studies reported very good inter-rater reliability for the MRC-SS with the ICC ranging from 0.83 to 0.99 . $(20,64,74)$ In terms of validity, Fan et al. (20) reported very good correlations between the MRC-SS with Maximal Inspiratory Pressure (MIP) $\left(R^{2}=0.94\right)$ and hand grip strength measured using dynamometry $\left(R^{2}=0.99\right)$ in Acute Lung Injury $(A L I)$ survivors 24 months post-hospital discharge. This indicates that MIP and hand grip strength reflects the same concept as the MRC-SS.

\subsubsection{Maximal Inspiratory Pressure (MIP)}

Maximal inspiratory pressure (MIP) is a measure of respiratory muscle strength and endurance. The procedure used in various studies to measure MIP in mechanically ventilated patients entailed a unidirectional valve attached to the endotracheal tube and a manometer. Critically ill patients are disconnected from the ventilator for a short duration and MIP values are recorded. However, this procedure requires specialised equipment and is time consuming. Respiratory muscle endurance has been measured using the Fatigue Resistance Index (FRI) which compares MIP values before and after a two-minute loading challenge. (50) An alternative way to measure MIP and respiratory muscle endurance is through a mouth piece, containing a bacterial filter. To determine respiratory muscle strength, patients are asked to exhale as much as they can, followed by taking a deep breath. To determine respiratory muscle endurance, the inspiratory load is set at a load between $30 \%-50 \%$ of the patients MIP and thereafter, patients are asked to continue 
breathing against a resistance for three to seven minutes. (75) This method has been extensively used in the chronic obstructive pulmonary disease (COPD) population and was used in this study to determine respiratory muscle strength and endurance in critically ill patients.

\subsection{Reliability and validity}

Medrinal et al. (76) reported that similar MIP results were produced between patients who were intubated and extubated and thus, concluded that respiratory muscle strength measurements taken in both intubated and extubated patients are reliable. In a study conducted by Formiga et al. (77), they reported a very good test-retest reliability for MIP $(I C C=0.98)$, whereas with convergent validity, no significant relationship was found between MIP and the six-minute walk test (6-MWT). However, these results should be interpreted with caution as the study population were patients diagnosed with COPD.

\subsubsection{Dynamometry}

Dynamometry is a standardised method used to evaluate muscle strength of the upper and lower limbs. It consists of two outcome measures: Hand-grip dynamometry assesses grip strength only whereas hand-held dynamometry assesses muscle strength of the upper limbs and lower limbs by performing a maximal isometric contraction. Dynamometry records the force produced by loading through tension or compression and is measured in units of weight such as newtons $(\mathrm{N})$ or kilograms $(\mathrm{kg}) .(78)$

Alternative measurement tools used to assess muscle strength include electrophysiological measurements and manual muscle testing. Electrophysiological measurements are time consuming, expensive, technically challenging and are not always readily available in all ICUs. With regards to manual muscle testing, when an MRC-SS exceed grade 3 (movement against gravity), it loses much of its ability to discriminate between gradations of strength. (79) Therefore, additional assessment tools such as dynamometry are necessary to 
measure muscle strength of upper and lower limbs muscles that are strong enough to overcome gravity. (79)

\subsection{Reliability and validity}

Dynamometry has shown to be a reliable tool to assess muscle strength of the upper and lower limbs in awake and cooperative critically ill patients. Baldwin et al. (4) reported a very good inter-rater reliability for bilateral handgrip force (Right upper limb: ICC $=0.92$, Left upper limb: $I C C=0.89$ ) and for bilateral isometric knee extension force (Right lower limb: $I C C=0.84$, Left lower limb: $I C C=0.79$ ). In addition, the same study obtained a very good test-retest reliability for bilateral handgrip force (Right upper limb: ICC $=0.92$, Left upper limb: $I C C=0.86$ ) and for bilateral isometric knee extension force (Right lower limb: ICC = 0.90, Left lower limb: $I C C=0.91)$. (4) Baldwin et al. (4) also reported that a mean change of $2.8(7.8) \mathrm{kg}$ for handgrip force and $2.6(7.1) \mathrm{kg}$ for knee extension force may be a requisite from a patient's baseline measurements to reflect accurate force changes. A study conducted by Hermans et al. (64) also demonstrated a very good inter-rater reliability and reproducibility for bilateral handgrip force (Right upper limb: ICC $=0.93$, Left upper limb: ICC $=0.97$.

\subsubsection{Physical function}

\subsubsection{The Chelsea Critical Care Physical Assessment (CPAx) tool}

The Chelsea Critical Care Physical Assessment (CPAx) tool is an outcome measure designed to assess physical function in critically ill patients (80) and is used clinically in South Africa by physiotherapists. (81) The CPAx tool consists of 10 domains of physical ability such as respiratory function, cough, bed mobility, supine to sitting on the edge of the bed, dynamic sitting, sit to stand, standing balance, transferring from bed to chair, stepping and grip strength. (80) Each of the 10 domains are graded on a six-point scale from zero being complete dependence to five being complete independence. (82) Individual values are collated and a total score out of 50 is then obtained. The score can be depicted on a radar 
chart, showing a patient's functional capabilities and highlighting problem areas requiring improvement.

\subsection{Reliability and validity}

The CPAx tool is simple to use, has a limited floor and ceiling effect, and reliability testing has shown strong internal consistency and inter-rater reliability. $(80,82,83)$ Corner et al. $(80)$ demonstrated a moderate internal consistency $(I C C=0.80)$, and a very good inter-rater reliability $(I C C=0.99)$ between five physiotherapists. The results for construct validity showed strong positive significant correlations between pre-admission CPAx scores and the ICU discharge CPAx scores with the physical component of the Short Form 36 (SF-36) score $(r h o=0.72, p=0.01$; rho $=0.84, p=0.01)$, but a weak positive correlation with the mental component of the SF-36 score ( $r h o=0.12, p=0.72$; rho $=0.02, p=0.95$ ).

\subsubsection{2 de Morton Mobility Index (DEMMI)}

The de Morton Mobility Index (DEMMI) is a measure of mobility and has been used in acute, subacute, and/or community settings. (84) The DEMMI was originally developed to assess mobility within the International Classification Functioning, Disability and Health (ICF) domain in elderly patients admitted to hospital. It includes assessments such as bed mobility, transfers, ambulation and jumping, making it feasible and relevant to evaluate recovery in critically ill patients throughout the trajectory i.e., during and after an ICU stay. (85) Therefore, in this study the DEMMI was used to determine critically ill survivors' physical function at hospital discharge.

\subsection{Reliability and validity}

Sommers et al. (85) reported that the DEMMI is a feasible, reliable and a moderately valid outcome measurement tool. The tool also has a minimal floor or ceiling effect when measuring mobility within the ICF domain in critically ill patients. A very good inter-rater reliability was obtained at ICU admission $(I C C=0.93)$ and at ICU discharge $(I C C=0.97)$ where two physiotherapists performed the DEMMI simultaneously and independently. A 
moderate intra-rater reliability $(I C C=0.68)$ was obtained when measurements for the DEMMI were conducted at ICU admission and repeated by both physiotherapists within onehour of assessment.

Furthermore, the study conducted by Sommer et al. (85) reported that the DEMMI showed a moderate convergent correlation with Katz Index of Independence in Activities of Daily Living (Katz ADL), the Barthel Index (BI) and Manual Muscle Testing (MMT) at both ICU admission $(r h o=0.56, r h o=-0.45, r h o=0.57)$, and at ICU discharge $(r h o=0.75$, rho $=-0.76$, rho $=$ 0.63). This indicates that the Katz ADL, the BI and MMT reflects a similar construct as the DEMMI tool and therefore, produces similar results.

\subsubsection{Six-minute walk test (6-MWT)}

The Six-Minute Walk Test (6-MWT) is a standardised outcome measure used to assess functional exercise capacity and has been validated in patients with a range of chronic conditions. The 6-MWT has also shown to be safe in critically ill survivors at hospital discharge $(86,87)$ and to be a useful measure for assessing recovery in a critically ill population. The test measures the distance that a patient can walk on a 30-metre flat, unimpeded walkway for a period of six-minutes. (88) Patients may walk at their own pace and are allowed to stop and rest during the test. The 6-MWT provides integrated information of all the systems involved during exercise, including the pulmonary, cardiovascular and neuromuscular systems. (88) It also provides important clinical outcomes in patients with chronic respiratory disease. (88)

\subsection{Reliability and validity}

To date, no psychometric evaluation of the 6-MWT in an acute hospital setting has been undertaken. A study conducted by Alison et al. (86) reported that it was important that critically ill survivors perform two 6-MWTs at each assessment in order to give an accurate reflection of the change in functional exercise capacity over time. 
The 6-MWT has excellent responsiveness and reliability, and a low to moderate content validity in patients with heart failure. Demers et al. (89) demonstrated a good test-retest reliability (ICC of 0.85). With regards to construct validity, the same study by Demers et al. (89) found that the 6-MWT was weakly inversely correlated with disease-specific quality of life $(r=-0.26, p=.0001)$ and moderately inversely correlated with the New York Heart Association-Functional Classification (NYHA-FC) $(r=-0.43, p=.001)$. Furthermore, a minimal clinically important difference (MCID) of 20 to 30 metres or $3 \%$ to $5 \%$ predicted can help clinicians using the 6-MWT to assess and interpret the efficacy of research interventions amongst patients with Acute Respiratory Failure (ARF) or Acute Respiratory Distress Syndrome (ARDS) post-hospital discharge. (90)

\subsubsection{Health-related quality of life (HRQoL)}

Developed by the EuroQol Group, the EQ-5D-5L is a questionnaire used to assess healthrelated quality of life (HRQoL). It comprises of two parts: the EQ-5D self-classifier and EQVAS. The EQ-5D self-classifier consists of five dimensions of health such as mobility, selfcare, usual activities, pain or discomfort and anxiety or depression. Each dimension is divided into five response levels: no problems, slight problems, moderate problems, severe problems and extreme problems. The EQ-VAS is used to record a patient perception of their health status using a Visual Analogue Scale (VAS). The scale is graded from zero (the worst health) to 100 (the best health). (91)

\subsection{Reliability and validity}

Li et al. (92) reported a very good test-retest reliability across the five dimensions of the EQ5D-5L ranging from $86.3 \%$ to $94.1 \%$. In terms of convergent reliability, the same study by $\mathrm{Li}$ et al. (92) found moderate inverse correlations between the physical domain of the World Health Organisation Quality of Life- BREF (WHOQOL-BREF) questionnaire with the mobility $(r h o=-0.55)$, the self-care $(r h o=-0.52)$ and the pain/discomfort dimensions of the EQ-5D- 
5L. Similarly, a moderate inverse correlation was found between the psychological domain of the WHOQOL-BREF and the anxiety/depression dimension of the EQ-5D-5L (rho = 0.66).

\subsection{Data collection and management}

\subsubsection{Pilot study}

A pilot study was conducted preceding the commencement of the primary research study. Both intra-rater reliability and inter-rater reliability were established for the following ultrasound measurements: Tdi at end-inspiration (ICC $=0.85 ;$ ICC $=0.94)$, Tdi at endexpiration $(I C C=0.96 ; I C C=0.90)$, RF muscle thickness $(I C C=0.99 ; I C C=0.96)$, VI muscle thickness $(\mathrm{ICC}=0.99 ; \mathrm{ICC}=0.95)$, RF muscle echogenicity $(C C=0.97 ; \mathrm{ICC}=0.20)$ and VI muscle echogenicity $(I C C=0.84 ;$ ICC $=0.94)$ (Addendum I).

\subsubsection{Baseline data}

Baseline data was extracted on the day of screening from the medical file and charts for each participant. Data included participant information, demographics, current medical history, past medical history, the Acute Physiologic Assessment and Chronic health Evaluation II (APACHE II) score, the Sequential Organ Failure Assessment (SOFA) score, and the daily assessment which consisted of: Number of hours intubated, mode of ventilation, pressure support, positive end-expiratory pressure (PEEP), peak pressure, dynamic compliance, medication, sedative agent and level of sedation/consciousness. All data was captured electronically onto REDCap. The APACHE II scores were available in the medical file of each participant and the SOFA score was calculated by the PI. Refer to Addendum $\mathrm{J}$ for examples of the REDCap forms.

\subsubsection{Measures of muscle structure, muscle function, physical function and health- related quality of life (HRQoL)}

All measurements of muscle structure and function, physical function and HRQoL were taken by the $\mathrm{PI}$ in a randomised order. Data collection took place from Monday to Friday. 
Participants were followed up from ICU admission until hospital discharge. If participants were planned for discharge over the weekend, data collection was done on the Friday prior to the participant being discharged. However, if a participant was discharged over the weekend unexpectedly without data collection being completed, that participant was regarded as lost to follow-up.

Table 3.1: Summary of time points for assessments of muscle structure, muscle function, physical function and HRQoL

\begin{tabular}{|c|c|c|c|c|c|c|}
\hline & Day 1 & Day 2 & Day 3 & $\begin{array}{c}\text { Participant } \\
\text { Awake in ICU }\end{array}$ & $\begin{array}{c}\text { ICU } \\
\text { Discharge }\end{array}$ & $\begin{array}{c}\text { Hospital } \\
\text { Discharge }\end{array}$ \\
\hline Ultrasonography: Diaphragm Muscle & $\checkmark$ & $\checkmark$ & $\checkmark$ & & & \\
\hline $\begin{array}{c}\text { Ultrasonography: Rectus Femoris and } \\
\text { Vastus Intermedius Muscles }\end{array}$ & $\checkmark$ & $\checkmark$ & $\checkmark$ & & & \\
\hline MRC-SS & & & & $\checkmark$ & $\checkmark$ & $\checkmark$ \\
\hline CPAX & & & & $\checkmark$ & $\checkmark$ & $\checkmark$ \\
\hline MIP & & & & & $\checkmark$ & $\checkmark$ \\
\hline Dynamometry & & & & & & $\checkmark$ \\
\hline DEMMI & & & & & & $\checkmark$ \\
\hline 6 MWT & & & & & & \\
\hline EQ-5D-5L & & & & & & $\checkmark$ \\
\hline
\end{tabular}




\subsubsection{Measures of muscle structure and function}

\subsection{Ultrasonography of the diaphragm and quadriceps muscle}

Ultrasonography images were obtained within 24 hours of admission to the ICU each day for three days. All imaging was done using a Phillips Lumify portable ultrasound machine (Washington; United States of America (USA)) in B-mode, using a 7-12 MHz linear probe. Participants were positioned in a supine position with the head of the bed elevated to $30^{\circ}$ for imaging of the diaphragm muscle, and in a supine position with their right knee in passive extension, neutral rotation and the great toe pointing towards the ceiling for imaging of the quadriceps muscle. (26) A water-soluble gel was applied to the ultrasound probe for acoustic contact without depressing the dermal surface.

The right hemi-diaphragm muscle was imaged at the zone of apposition (ZOA) with the ultrasound probe placed against the chest wall, on the right anterior axillary or mid-axillary line between the eighth and tenth intercostal space where a clear image was obtained. (68) The hemi-diaphragm was identified as a structure composed of three distinct layers: A nonechogenic central muscular layer bound by echogenic membranes, the peritoneum and the diaphragmatic pleura. (93) One video of the hemi-diaphragm muscle was captured during three consecutive tidal breaths, from inspiration to expiration for each participant. The RF and $\mathrm{VI}$ muscle were imaged anteriorly on the right thigh. The ultrasound probe was placed perpendicular to the long axis of the anterior thigh, two-thirds from the anterior superior iliac spine (ASIS) to the superior patella border. (26) One image of the RF and VI muscle was captured for each participant.

All ultrasonography images were captured directly onto the Phillips Lumify system, and subsequently exported without any alterations to a computer for further analysis. Image analysis was done using the image analysis software ImageJ (National Institutes of Health, Bethesda, MD). (25) The following measures of muscle size and echogenicity were 
obtained: Tdi at end-inspiration, Tdi at end-expiration, DTF, RF muscle thickness, RF CSA, RF echogenicity, VI muscle thickness and VI echogenicity.

During analysis, the PI captured three images from end-inspiration to end-expiration for the three consecutive tidal breaths. The average Tdi of each image was calculated first by taking measurements at three random points on the diaphragm at end-inspiration and at endexpiration. The average measurement obtained for end-inspiration and end-expiration was then used to calculate the DTF. Change in Tdi were classified in the following categories: no change in Tdi of less than $10 \%(<10 \%)$ and an increase or decrease in Tdi of more than $10 \%(>10 \%)$.

A standard square of $20 \times 20 \mathrm{~mm}$ and a computer-assisted quantitative grayscale was used for the analysis of echogenicity of the RF and VI muscle. If the area to be analysed was smaller than $20 \times 20 \mathrm{~mm}$, the largest possible square within the anatomic boundaries of the muscle was then used for analysis. (26) Muscle thickness of the RF and VI muscles were measured at three random points along the muscle belly. RF CSA was measured along the border of the muscle belly. All measures were performed in triplicate, with the average of the scores used in the final analysis.

\subsection{Medical Research Council-Sum Score (MRC-SS)}

Peripheral muscle strength was assessed using the Medical Research Council-Sum Score (MRC-SS). The first MRC-SS measurement was performed when the participant was awake in the ICU and thereafter at ICU discharge and at hospital discharge. Prior to the commencement of the voluntary muscle strength assessment, evaluation of the participant level of cooperation was first determined by the Standardised Five Questions (S5Q). (79) Participants were positioned in a supine position with the head of the bed elevated to $45^{\circ}$ to perform movements against gravity (MRC-SS $\geq 3$ ), and/or the head of the bed elevated to $10^{\circ}$ to perform movements with elimination of gravity (MRC-SS $\leq 3$ ). (79) Three muscle 
groups of upper limbs (shoulder abduction, elbow flexion and wrist extension) and three muscle groups of the lower limbs (hip flexion, knee extension and ankle dorsiflexion) was assessed bilaterally. (79) The PI first demonstrated the movement passively to the participant, after which the participant actively performed the movement. A six-point grading scale ranging from zero (no visible contraction) to five (normal power) with a composite score of 60 was used for the assessment of muscle strength. A score of $<48$ was indicative of ICU-AW. Individual scores for each muscle group of both the left and right upper and lower limbs as well as the overall composite MRC-SS was captured. (79)

Table 3.2: The MRC - SS grading scale

\begin{tabular}{|c|c|}
\hline Grade 0 & No contraction visible or palpable \\
\hline Grade 1 & Flicker of contraction visible or palpable, although no limb movement \\
\hline Grade 2 & Movement with gravity eliminated over almost full range of motion \\
\hline Grade 3 & Movement against gravity over almost full range of motion \\
\hline Grade 4 & Movement against moderate resistance over full range of motion \\
\hline Grade 5 & Movement against full resistance over full range of motion i.e., Normal power \\
\hline
\end{tabular}




\subsection{Maximal Inspiratory Pressure (MIP)}

Maximal inspiratory pressure (MIP) was measured at ICU discharge and at hospital discharge using the American Thoracic Society/European Respiratory Society (ATS/ERS) guidelines. (6) Respiratory muscle endurance was only performed at hospital discharge and not at ICU discharge as participants may have been fatigued post-extubation. Both outcomes were measured using the POWERbreathe KH1 (Southampton; United Kingdom) device. Participants were instructed to sit comfortably on the chair provided, with their backs firmly positioned against the back-rest and their feet placed firmly on the floor.

To determine respiratory muscle strength, a mouthpiece containing a bacterial filter was placed in the participant's mouth after which he or she was asked to exhale as much as they could, followed by taking a deep breath. (75) To determine respiratory muscle endurance, the inspiratory load was initially set at a load of $50 \%$ of the participants MIP and this allowed participants to continue breathing against a resistance for three to seven minutes. (75) Participants were asked to breathe against a submaximal inspiratory load provided by the POWERbreathe KH1 (Southampton; United Kingdom) device until task failure. (75) The following data was recorded: three measurements for MIP, and for respiratory muscle endurance, the time it took for completion of the test, number of breaths, average mean load and the average mean power.

\subsection{Dynamometry}

Peak isometric knee extension force and hand grip strength was assessed at hospital discharge using the Lafayette Manual Muscle Test System (Lafayette; USA) and the Jamar hydraulic handgrip dynamometer (Chicago; USA). At first, each participant was asked which were their dominant upper limb and lower limb. To quantify knee extension force, participants were seated on a chair with their hips and knees flexed at $90^{\circ}$. The PI passively demonstrated the required movement and then asked the participant to perform the movement actively. The dynamometer was placed on the anterior aspect of the lower limb, 
proximal to the ankle joint and the participant performed a maximal voluntary isometric contraction and maintained the contraction for at least three to five seconds. $(78,79)$

To quantify grip strength, participants were seated in a chair with their hips, knees and elbows all flexed to $90^{\circ}$ and their forearm supported on the armrests of a chair. (21) Participants were instructed to hold the hydraulic handgrip dynamometer and squeeze the lever with all their effort. The dominant limbs were assessed first, followed by the nondominant limbs. A rest period of $30-60$ seconds was given to participants between measurements. $(78,79)$ Three measurements for both the right and left upper and lower limbs were recorded, and the highest value was used for analysis.

\subsubsection{Measures of physical function}

\subsection{The Chelsea Critical Care Physical Assessment (CPAx) tool}

The Chelsea Critical Care Physical Assessment (CPAx) tool was performed when the participant was awake in the ICU and at ICU discharge. Prior to the commencement of the physical function assessment, evaluation of the participant level of cooperation was first determined by the S5Q. A score from zero to five was recorded for each of the ten domains of physical ability depending on the participant's level of function. (80)

\subsubsection{2 de Morton Mobility Index (DEMMI)}

The de Morton Mobility Index (DEMMI) was conducted at hospital discharge and used to assess 15 different aspects of mobility such as three bed based, three chair based, four static balance, two walking-related and three dynamic balance activities. (84) Participants were given a score from zero to two for each aspect of mobility with zero representing being unable to perform the activity and two being able to independently perform the activity. A total score out of 19 was converted with Rasch analysis to an interval score ranging from zero (poor mobility) to 100 (independent mobility). The individual scores for each category, the total score out of 19 and the interval score was recorded. 


\subsection{Six-Minute Walk Test (6-MWT)}

The Six-Minute Walk Test (6-MWT) was used to determine the functional exercise capacity of participants. It was conducted on a 30-metre unimpeded walkway prior to the participant being discharged from hospital according to the ATS guidelines. (94) Participants were instructed to walk as far as possible for six-minutes. Participants performed two 6-MWTs with a 30-minute rest interval between the two tests. No other tests nor measurements were performed during the 30-minute rest interval. If, however, a participant stopped walking during the test and refused to continue, the test was stopped and a chair was provided for the participant to sit on. The distance walked, the time and the reason for discontinuing the test was documented.

The following measurements were taken prior to the commencement of the test: Baseline heart rate, saturation ( $\mathrm{SpO} 2)$, dyspnoea and fatigue, if a participant was using an assistive device as well as the type of assistive device. After completion of each test, the following measurements were captured: Heart rate, $\mathrm{SpO} 2$, dyspnoea and fatigue, the number of laps and the distance walked. The six-minute walk distance (6-MWD) for each test and the best 6-MWD was used for analysis.

\subsubsection{Health-related quality of life (HRQoL)}

\subsection{EQ-5D-5L health questionnaire}

The EQ-5D-5L health questionnaire was completed at hospital discharge. Participants were asked to rate the five dimensions of health as either: no problems, slight problems, moderate problems, severe problems or extreme problems. The five dimensions of health included mobility, self-care, usual activities, pain or discomfort and anxiety or depression. Participants were also asked to rate their overall health on a VAS from $0-100$. The five dimensions of health and the overall health score were recorded. 


\subsection{Data analysis}

IBM SPSS version 27 (New York, United States of America) was used to analyse the data, in consultation with a statistician. Data was tested for normality using the Shapiro-Wilk test. Normally distributed data were presented as means and standard deviations (SD) and nonnormally distributed data were presented as medians and interquartile ranges (IQR). Correlations between measures of muscle structure, muscle function, physical function and HRQoL were assessed using Pearson's correlation if data were normally distributed or Spearman's correlation if data were non-normally distributed. The strength of the relationships at two different time points were classified as very weak, weak, moderate and strong and are represented in Table 3.3.

Table 3.3: Classification of correlation strengths

\begin{tabular}{|c|c|c|}
\hline & Pearson Correlation Coefficient & Spearman's Correlation Coefficient \\
\hline Very weak & $<0.3$ & $<0.3$ \\
\hline Weak & $0.3<r<0.5$ & $0.3<$ rho $<0.5$ \\
\hline Moderate & $0.5<r<0.7$ & $0.5<$ rho $<0.7$ \\
\hline Strong & $r>0.7$ & rho $>0.7$ \\
\hline
\end{tabular}




\section{Chapter 4: Results of the Primary Research Study}

The results of the primary research study will be presented in this chapter and coincides with the aim and research objectives of the primary research study.

\subsection{Study population}

Over a seven-month period ( $1^{\text {st }}$ August 2019 to $28^{\text {th }}$ February 2020), a total of 525 participants were screened for inclusion. Forty-five participants met the inclusion criteria and were recruited for the study from the medical and surgical ICUs of a Tertiary Academic Hospital in South Africa. Four hundred and eighty participants were excluded due to the following reasons: 1 . did not meet the inclusion criteria $(n=468) ; 2$. unplanned/early extubation before 48 hours of mechanical ventilation $(n=9)$; 3. withdrawn from the study $(n=2)$; and 4 . did not provide consent $(n=1)$. Figure 4.1 illustrates the flow of included participants.

Various measures of muscle structure, muscle function and physical function were included in this study. Due to safety criteria applied for individual measures, not all forty-five participants were measured for each of the individual measures. We will present the flow of participants as it pertains to each specific measure before we present the results of the measure. 
Participants Screened

$(n=525)$

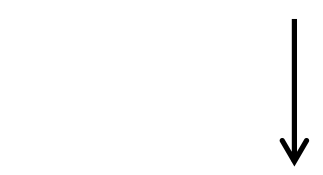

Participants who did not meet the inclusion criteria $(n=468)$

Participants eligible for inclusion

$(n=57)$

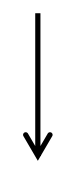

Participants did not provide consent

$(n=12)$

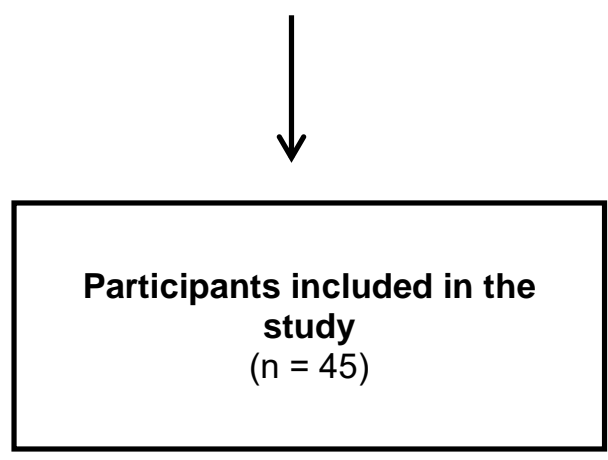

Participants included in the study

$(n=45)$
Not intubated

$$
(n=195)
$$

Plan to extubate within $48 \mathrm{hrs}$ $(n=48)$

Mechanically ventilated $>24 \mathrm{hrs}$

$$
(n=108)
$$

Younger than 18 years

$$
(n=7)
$$

Older than 65 year

$$
(n=30)
$$

Neurological conditions $(n=18)$

TBI/Head injury

$$
(n=23)
$$

Acute or chronic degenerative neuromuscular condition

$$
(n=2)
$$

Unstable fractures

$$
(n=12)
$$

Expected to die

$$
(n=10)
$$

Morbidly obese $(\mathrm{BMI}>40 \mathrm{~kg} / \mathrm{m} 2)$

$$
(n=13)
$$

Dressings hindering the application of the ultrasound probe

$$
(n=2)
$$

Figure 4.1: Flow chart of the included participants 


\subsection{Demographics and clinical characteristics}

\subsubsection{Gender}

Seventy-three percent $(n=33 / 45)$ of the participants were male (Table 4.1).

\subsubsection{Age}

The age of the participants ranged from 18 to 61 years, with a median (IQR) age of 34.5 (24.347.4) years (Figure 4.2; Table 4.1).

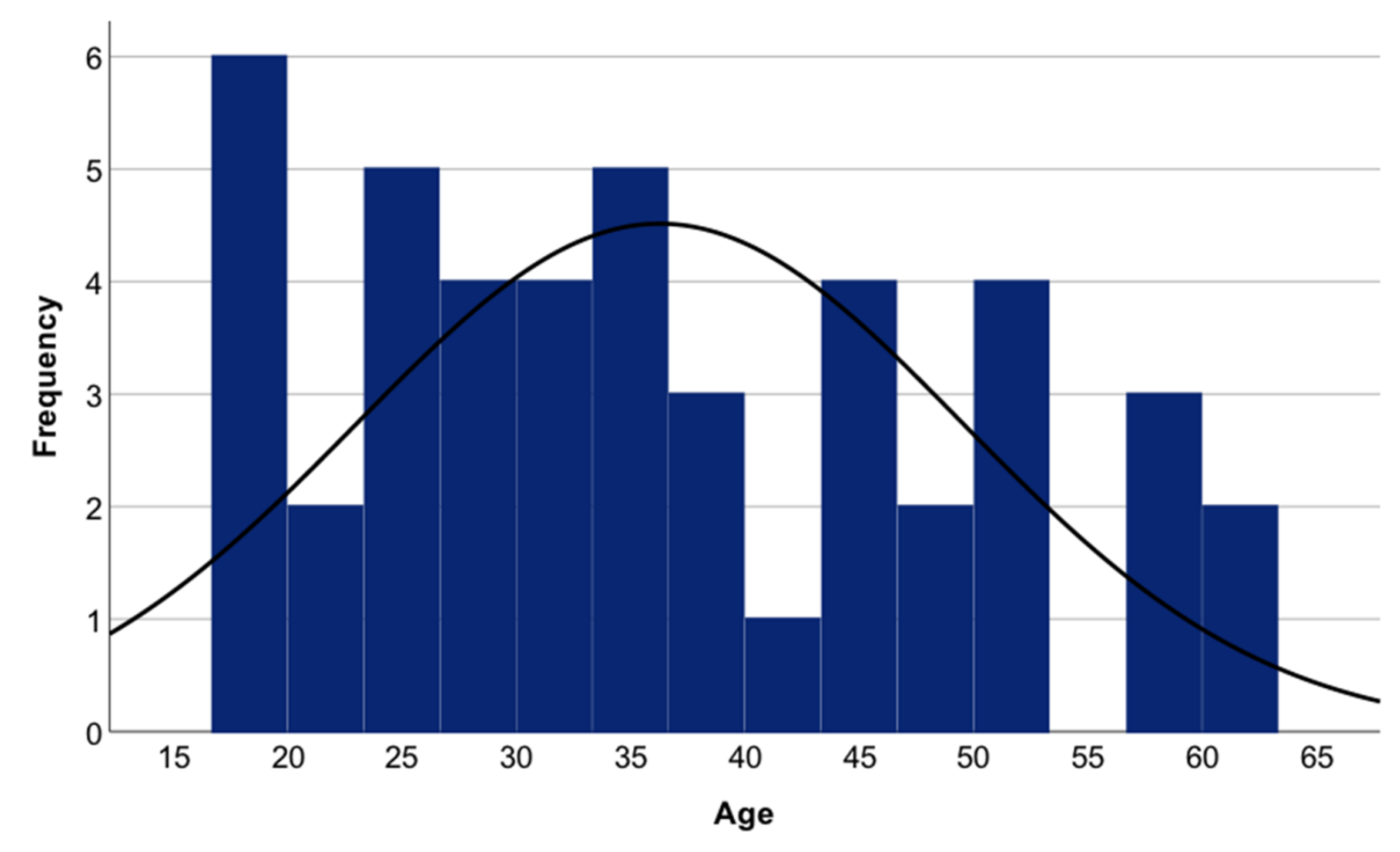

Figure 4.2: Distribution of the participant's age

\subsubsection{Severity of illness}

Two measures of illness severity were calculated. A wide variation in the severity of illness as measured with the APACHE II was noted, ranging between 3 to 39 points, with a median (IQR) score of $18.5(12.8-25.3)$ points, indicating that a large proportion of included participants were classified as severely ill (Figure 4.3; Table 4.1). Furthermore, a mean (SD) of $8.8 \pm 2.8$ points was found for the SOFA score, also classifying the included participants as severely ill as well (Figure 4.4; Table 4.1). 


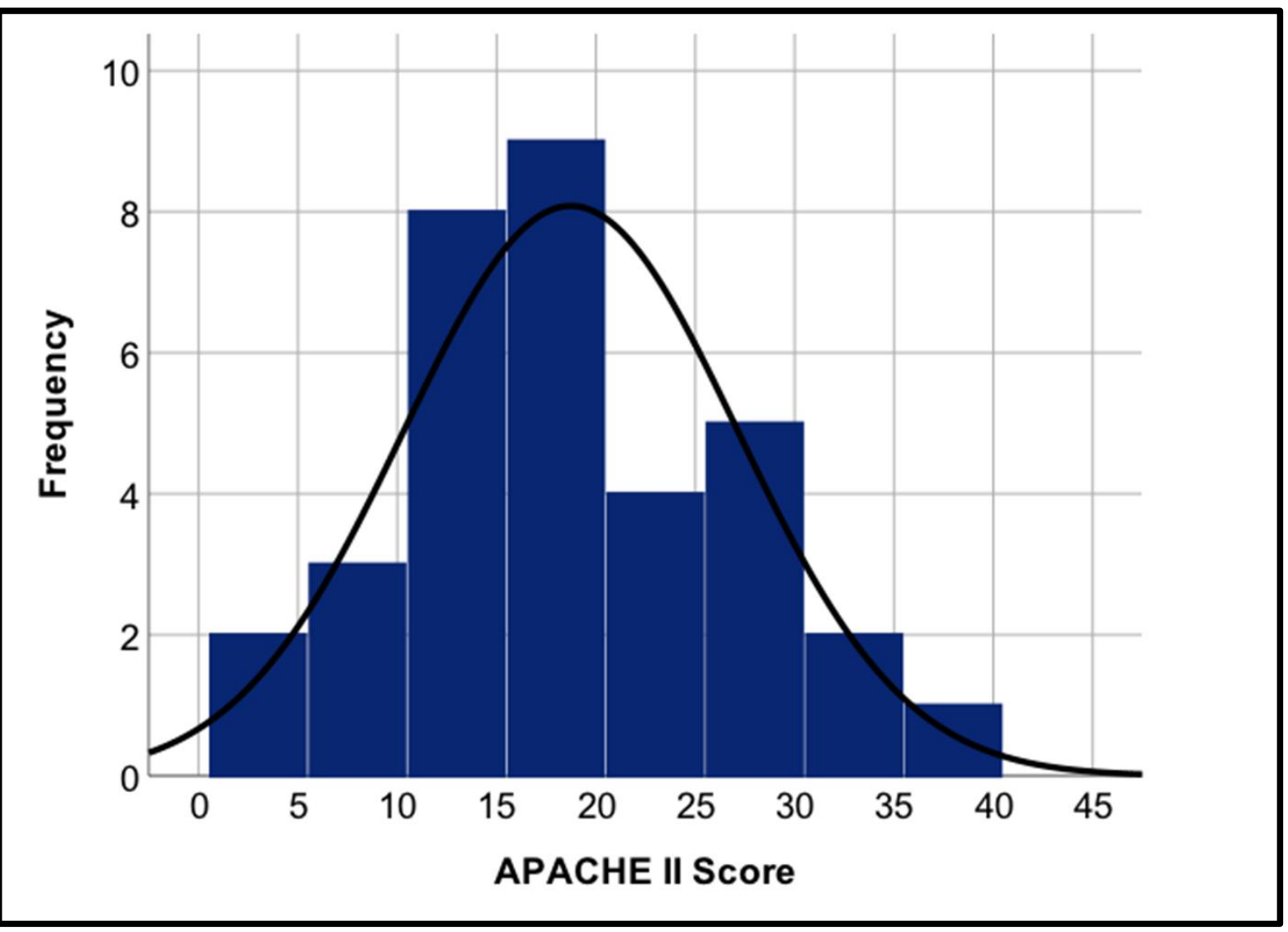

Figure 4.3: Distribution of the APACHE II score

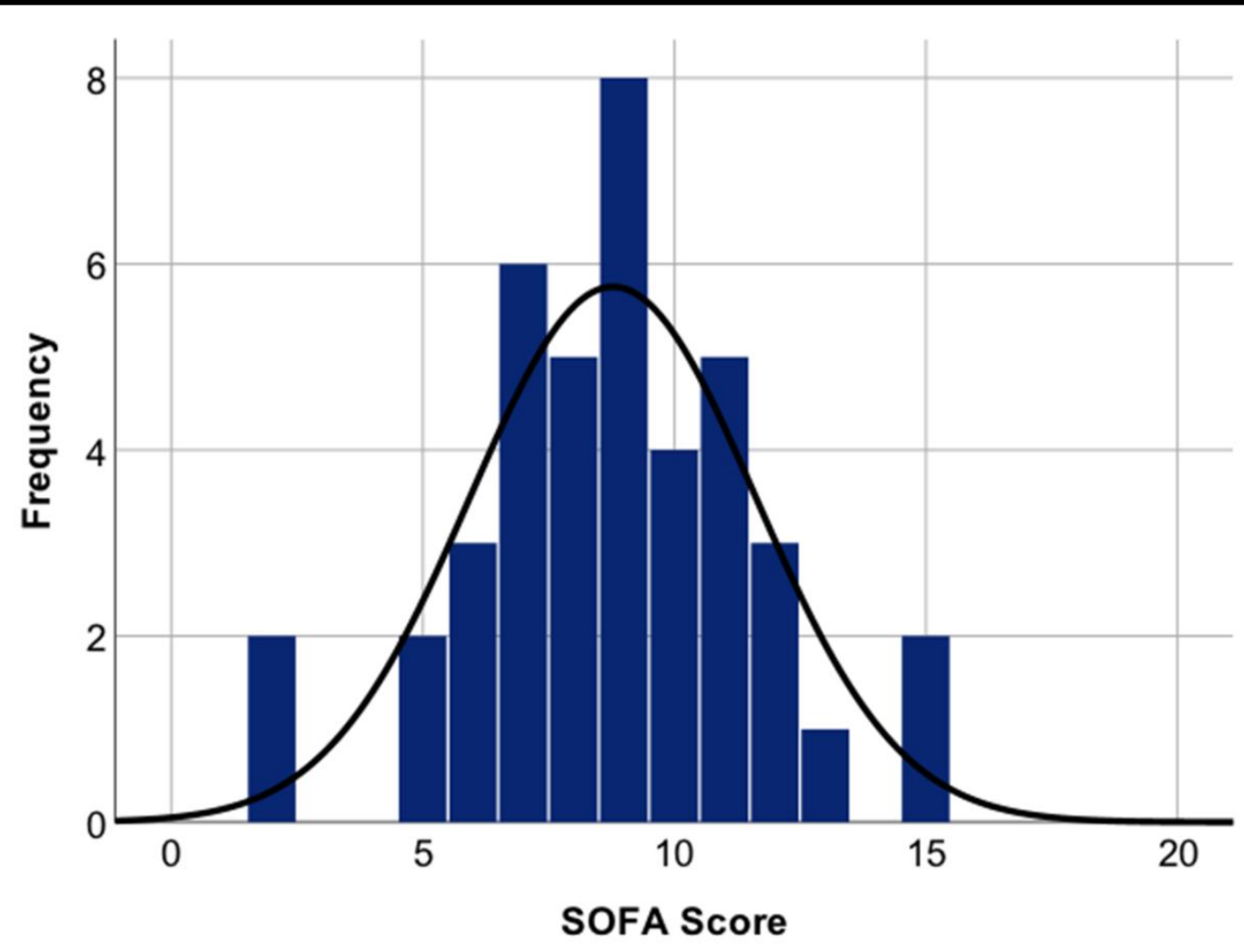

Figure 4.4: Distribution of the SOFA score 


\subsubsection{Admission diagnosis}

Majority $(n=41 / 45,91.1 \%)$ of the admissions to the ICUs were emergencies, with trauma $(n=$ $20 / 45,44.4 \%$ ) being the most common admission followed by medical ( $n=13 / 45,28.9 \%)$ and then surgical $(n=12 / 45,26.7 \%$ ) (Figure 4.5). Detail of admission diagnosis is summarised in Table 4.1

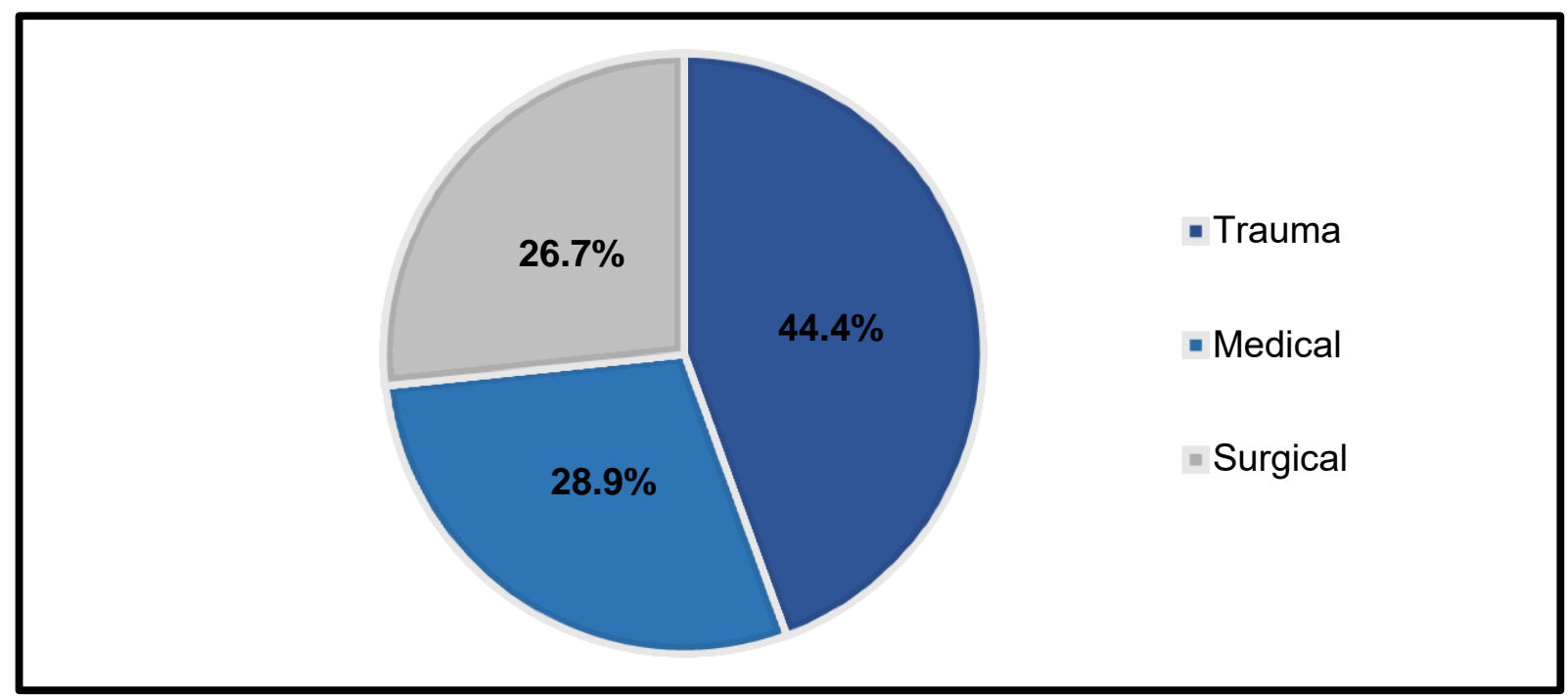

Figure 4.5: Percentage of trauma, medical and surgical admissions

\subsubsection{Employment status}

Thirty-seven $(82.2 \%)$ of the 45 participants were unemployed at the time of hospital admission (Table 4.1).

\subsubsection{Comorbidities and social history}

At ICU admission, the common comorbidities participants presented with were the retrovirus disease (RVD) and hypertension, and only a minority of participants were recorded for substance abuse (Table 4.1). 
Table 4.1: Demographics and clinical characteristics

\begin{tabular}{|c|c|c|}
\hline \multirow{2}{*}{ Characteristics } & Mean \pm SD / Median (IQR) / & \multirow{2}{*}{ Min - Max } \\
\hline & n (\%) & \\
\hline $\begin{array}{l}\text { Age (years) } \\
(\mathrm{n}=45)\end{array}$ & $34.5(24.3-47.4)$ & $18-61$ \\
\hline Male & $33(73.3 \%)$ & \\
\hline $\begin{array}{l}\text { Weight }(\mathrm{kg}) \\
(\mathrm{n}=36)\end{array}$ & $69(61.3-75.2)$ & $47-101$ \\
\hline \multicolumn{3}{|l|}{ Employed Before Admission } \\
\hline Yes & $8(17.8 \%)$ & \\
\hline No & $37(82.2 \%)$ & \\
\hline \multicolumn{3}{|l|}{ Severity Score } \\
\hline $\begin{array}{r}\text { APACHE II Score } \\
(\mathrm{n}=34)\end{array}$ & $18.5(12.8-25.3)$ & $3-39$ \\
\hline $\begin{array}{r}\text { SOFA Score } \\
(\mathrm{n}=41) \\
\end{array}$ & $8.8 \pm 2.8$ & $2-15$ \\
\hline \multicolumn{3}{|l|}{ Admission Type } \\
\hline Emergency & $41(91.1 \%)$ & \\
\hline Elective & $4(8.9 \%)$ & \\
\hline \multicolumn{3}{|l|}{ Admissions } \\
\hline Trauma & $20(44.4 \%)$ & \\
\hline Abdominal trauma & $3(6.7 \%)$ & \\
\hline Upper limb fractures & $3(6.7 \%)$ & \\
\hline Lower limb fractures & $2(4.4 \%)$ & \\
\hline Rib fractures & $1(2.2 \%)$ & \\
\hline Other & $6(13.3 \%)$ & \\
\hline Medical & $13(28.9 \%)$ & \\
\hline Pneumonia & $7(15.6 \%)$ & \\
\hline Sepsis & $2(4.4 \%)$ & \\
\hline Asthma & $1(2.2 \%)$ & \\
\hline COPD & $1(2.2 \%)$ & \\
\hline Cardiac arrest & $1(2.2 \%)$ & \\
\hline Other & $3(6.7 \%)$ & \\
\hline Surgical & $12(26.7 \%)$ & \\
\hline Abdominal & $10(22.2 \%)$ & \\
\hline Other & $2(4.4 \%)$ & \\
\hline \multicolumn{3}{|l|}{ Comorbidities } \\
\hline RVD & $8(17.8 \%)$ & \\
\hline Hypertension & $6(13.3 \%)$ & \\
\hline Other & $6(13.3 \%)$ & \\
\hline Diabetes & $4(8.9 \%)$ & \\
\hline TB & $4(8.9 \%)$ & \\
\hline Asthma & $3(6.7 \%)$ & \\
\hline COPD & $2(4.4 \%)$ & \\
\hline Past TB & $2(4.4 \%)$ & \\
\hline \multicolumn{3}{|l|}{ Social History } \\
\hline Current Smokers & $11(24.4 \%)$ & \\
\hline Alcohol use & $8(17.8 \%)$ & \\
\hline Illicit Drug use & $2(4.4 \%)$ & \\
\hline
\end{tabular}

Values are represented as a mean $\pm \mathrm{SD}$, median (interquartile range), a number and percentage (\%), or as minimum and maximum; Kg - Kilograms; Other - Facial trauma, Organophosphate poisoning, Malaria, Reproductive surgery 


\subsection{Length of stay (LOS)}

The median (IQR) for ICU length of stay (LOS) was $3(1-5)$ days and for total hospital LOS was 7 $(3-11)$ days (Table 4.2).

Table 4.2: Length of stay (LOS)

\begin{tabular}{|c|c|c|}
\hline & Median (IQR) & Min - Max \\
\hline Time in Hospital Before ICU Admission & $1(1-2)$ & $1-14$ \\
ICU Length of Stay (LOS) (d) & $3(1-5)$ & $1-25$ \\
Total hospital Length of Stay (LOS) (d) & $7(3-11)$ & $1-49$ \\
\hline
\end{tabular}

Values are represented as a median (interquartile range), or as minimum and maximum; $d$ - days; LOS - Length of Stay

\subsection{ICU management}

The care participants received while in ICU will be presented below.

\subsubsection{Ventilation mode, settings and measures}

On day one, majority of the participants were ventilated on the BiPAP mode $(n=20 / 45,44.4 \%$ ). Thereafter, on day two and three, CPAP was the common ventilation mode utilised for majority of the participants. The settings and measures for ventilation such as positive end-expiratory pressure (PEEP), pressure support, peak pressure and dynamic compliance were recorded daily for three days prior to conducting ultrasonography. The median (IQR) for the length of ventilation (LOV) was 3 (1-6) days. These results are represented in Table 4.3. 
Table 4.3: Mode, settings and measures of ventilation

\begin{tabular}{|c|c|c|c|}
\hline \multicolumn{4}{|c|}{ Mode of Ventilation } \\
\hline \multirow{2}{*}{\multicolumn{2}{|c|}{ Length of Ventilation (LOV) (d) }} & Median (IQR) & Min - Max \\
\hline & & $3(1-6)$ & $1-27$ \\
\hline & \multicolumn{3}{|c|}{ Number of Participants } \\
\hline & $\begin{array}{c}\text { Day } 1 \\
(n=45) \\
\end{array}$ & $\begin{array}{c}\text { Day 2 } \\
(n=31)\end{array}$ & $\begin{array}{c}\text { Day } 3 \\
(n=25) \\
\end{array}$ \\
\hline CPAP & 13 & 21 & 19 \\
\hline BiPap & 20 & 8 & 6 \\
\hline PC SIMV & 10 & 2 & 0 \\
\hline VC SIMV & 1 & 0 & 0 \\
\hline$A P R V$ & 1 & 0 & 0 \\
\hline \multicolumn{4}{|c|}{ Ventilation Settings and Measures } \\
\hline & $\begin{array}{c}\text { Day } 1 \\
(\mathrm{n}=45)\end{array}$ & $\begin{array}{c}\text { Day } 2 \\
(\mathrm{n}=\mathbf{3 1})\end{array}$ & $\begin{array}{c}\text { Day } 3 \\
(n=25)\end{array}$ \\
\hline $\begin{array}{r}P E E P \\
(\mathrm{cmH} 2 \mathrm{O}) \\
\text { median }(I Q R) \\
\end{array}$ & $10(9-12)$ & $10(8-12)$ & $8(8-10)$ \\
\hline $\begin{array}{r}\text { Pressure Support } \\
\text { (cmH2O) } \\
\text { median (IQR) }\end{array}$ & $12(9.5-14)$ & $10(8-14)$ & $10(8-12)$ \\
\hline $\begin{array}{r}\text { Peak Pressure } \\
\text { (Mode over } 24 \text { hours) } \\
\text { median (IQR) }\end{array}$ & $22(20-26)$ & $20(15-24)$ & $19(17-24)$ \\
\hline $\begin{array}{r}\text { Dynamic Compliance } \\
(\mathrm{L} / \mathrm{cmH} \mathrm{H} O) \\
\text { median }(I Q R)\end{array}$ & $46(30.5-58.8)$ & $44.5(33-71.3)$ & $48(38-72.9)$ \\
\hline
\end{tabular}

Values are represented as a median (interquartile range), as a number and percentage (\%), or as minimum and maximum; LOV Length of Ventilation

\subsubsection{Medication and sedation}

Only a minority of participants were sedated, received corticosteroids and/or insulin during the course of their ICU stay. Majority of the participants were exposed to sedation for only one day, with Propofol and Fentanyl being the two most common sedatives used. There were five participants that were administered corticosteroids for more than three days (Table 4.4). The level of sedation according to the Richmond Agitation and Sedation Scale (RASS) varied amongst the participants between day one and day three. Participants mainly scored either "0" ( $n=10 / 45$, 22.2\%), “-1" ( $n=9 / 45,20 \%)$ or "-5" ( $n=9 / 45,20 \%)$ on day one, and "0" on day two ( $n=13 / 31$, $41.9 \%)$ and day three $(n=14 / 29,48.3 \%)$ according to the RASS scale (Addendum K). 
Table 4.4: Number of participants that were exposed to sedation, corticosteroids and insulin during the ICU stay

\begin{tabular}{|r|c|c|c|}
\hline \multirow{2}{*}{} & \multicolumn{3}{|c|}{ Number of Participants } \\
\cline { 2 - 4 } & Once off & < 3 Days & > 3 Days \\
\hline Sedation & & & 1 \\
\hline Morphine & 3 & 2 & \\
\hline Propofol & 5 & 2 & \\
\hline Fentanyl & 4 & & 5 \\
\hline Corticosteroids & 1 & 1 & 1 \\
\hline Insulin & 3 & 3 & \\
\hline
\end{tabular}

\subsection{Ultrasonography of the Respiratory and Peripheral muscles}

\subsubsection{Respiratory muscles}

Participants Included $(n=45)$

$\downarrow$

\begin{tabular}{|c|c|c|c|c|c|}
\hline \multicolumn{6}{|c|}{ Respiratory muscles } \\
\hline \multicolumn{2}{|r|}{$\downarrow$} & \multicolumn{2}{|r|}{$\downarrow$} & \multicolumn{2}{|r|}{$\downarrow$} \\
\hline \multicolumn{2}{|r|}{ Day 1} & \multicolumn{2}{|r|}{ Day 2} & \multicolumn{2}{|r|}{ Day 3} \\
\hline $\begin{array}{l}\text { Respiratory } \\
\quad(n=36)\end{array}$ & $\begin{array}{l}\text { Ultrasonography device } \\
\text { not available } \\
(n=1) \\
\text { ICD Inhibiting Imaging } \\
(n=8)\end{array}$ & $\begin{array}{c}\text { Respiratory } \\
\quad(n=25)\end{array}$ & $\begin{array}{l}\text { Ultrasonography device } \\
\text { not available } \\
(n=1) \\
\text { Died } \\
(n=8) \\
\text { ICD Inhibiting Imaging } \\
(n=9) \\
\text { Unplanned/ Early } \\
\text { extubation } \\
(n=2)\end{array}$ & $\begin{array}{c}\text { Respiratory } \\
\quad(n=22)\end{array}$ & $\begin{array}{l}\text { Ultrasonography device } \\
\text { not available } \\
(n=1) \\
\text { Died } \\
(n=8) \\
\text { ICD inhibiting imaging } \\
(n=10) \\
\text { Unplanned/ Early } \\
\text { extubation } \\
(n=2) \\
\text { Successfully extubated } \\
(n=1) \\
\text { Medically unstable } \\
(n=1)\end{array}$ \\
\hline
\end{tabular}

The mean (SD) on day one for diaphragm thickness (Tdi) at end-inspiration and at end-expiration was $2.7 \pm 0.8 \mathrm{~mm}$ and $2.4 \pm 0.7 \mathrm{~mm}$ respectively, and subsequently declined on day two and day three (Table 4.5). 
Table 4.5: Ultrasonography of the Respiratory muscles

\begin{tabular}{|c|c|c|c|}
\hline Respiratory muscles & $\begin{array}{c}\text { Day 1 } \\
(\mathbf{n = 3 6 )}\end{array}$ & $\begin{array}{c}\text { Day 2 } \\
(\mathbf{n}=\mathbf{2 5})\end{array}$ & $\begin{array}{c}\text { Day 3 } \\
(\mathbf{n}=\mathbf{2 2})\end{array}$ \\
\hline $\begin{array}{c}\text { Diaphragm thickness (Tdi) at } \\
\text { end-inspiration } \\
(\mathrm{mm})\end{array}$ & $2.7 \pm 0.8$ & $2.5 \pm 0.7$ & $2.4 \pm 0.6$ \\
$\begin{array}{c}\text { Diaphragm thickness (Tdi) at } \\
\text { end-expiration } \\
(\mathrm{mm})\end{array}$ & $2.4 \pm 0.7$ & $2.3 \pm 0.7$ & $2.2 \pm 0.6$ \\
\hline
\end{tabular}

Values are represented as mean $\pm \mathrm{SD} ; \mathrm{mm}$ - millimetres

However, a sub-analysis indicated that there was a variation in how the Tdi changed over the three days for both end-inspiration and end-expiration. The results are presented in Table 4.6 and Table 4.7.

\subsubsection{Diaphragm thickness (Tdi) at end-inspiration}

At end-inspiration, $17 / 22$ of the participants $(77 \%)$ presented with no change in Tdi on both day two and day three, $1 / 22$ participant (5\%) presented with a more than $10 \%$ increase in Tdi on day two, but no change in Tdi on day three, and 4/22 participants (18\%) presented with no change in Tdi on day two, but a more than $10 \%$ decrease in Tdi on day three (Figure 4.6). The mean (SD) for the Tdi at end-inspiration for the three categories between day one and day three are represented in Table 4.6.

Table 4.6: Tdi at end-inspiration for the three categories

\begin{tabular}{|c|c|c|c|}
\hline \multicolumn{3}{|c|}{ Tdi at end-inspiration } \\
\hline & Day 1 & Day 2 & Day 3 \\
\hline $\begin{array}{c}\text { Baseline } \\
(\mathrm{n}=22)\end{array}$ & $\begin{array}{c}2.6 \pm 0.6 \\
(\mathrm{~mm})\end{array}$ & & \\
\hline $\begin{array}{c}\text { Group 1 } \\
\text { No change }<\% \\
(\mathrm{n}=17)\end{array}$ & & $\begin{array}{c}2.5 \pm 0.6 \\
(\mathrm{~mm})\end{array}$ & $\begin{array}{c}2.5 \pm 0.6 \\
(\mathrm{~mm})\end{array}$ \\
\hline $\begin{array}{c}\text { Group 2 } \\
\text { No change }<10 \%-\text { Decrease }>10 \% \\
(\mathrm{n}=4)\end{array}$ & & $\begin{array}{c}2.4 \pm 0.7 \\
(\mathrm{~mm})\end{array}$ & $\begin{array}{c}1.9 \pm 0.6 \\
(\mathrm{~mm})\end{array}$ \\
\hline $\begin{array}{c}\text { Group 3 } \\
\text { Increase }>10 \% \text { No change }<10 \% \\
(\mathrm{n}=1)\end{array}$ & & $\begin{array}{c}2.9 \\
(\mathrm{~mm})\end{array}$ & $\begin{array}{c}2.7 \\
(\mathrm{~mm})\end{array}$ \\
\hline
\end{tabular}

Values are represented as mean $\pm \mathrm{SD}$; $\mathrm{mm}$ - millimetres 


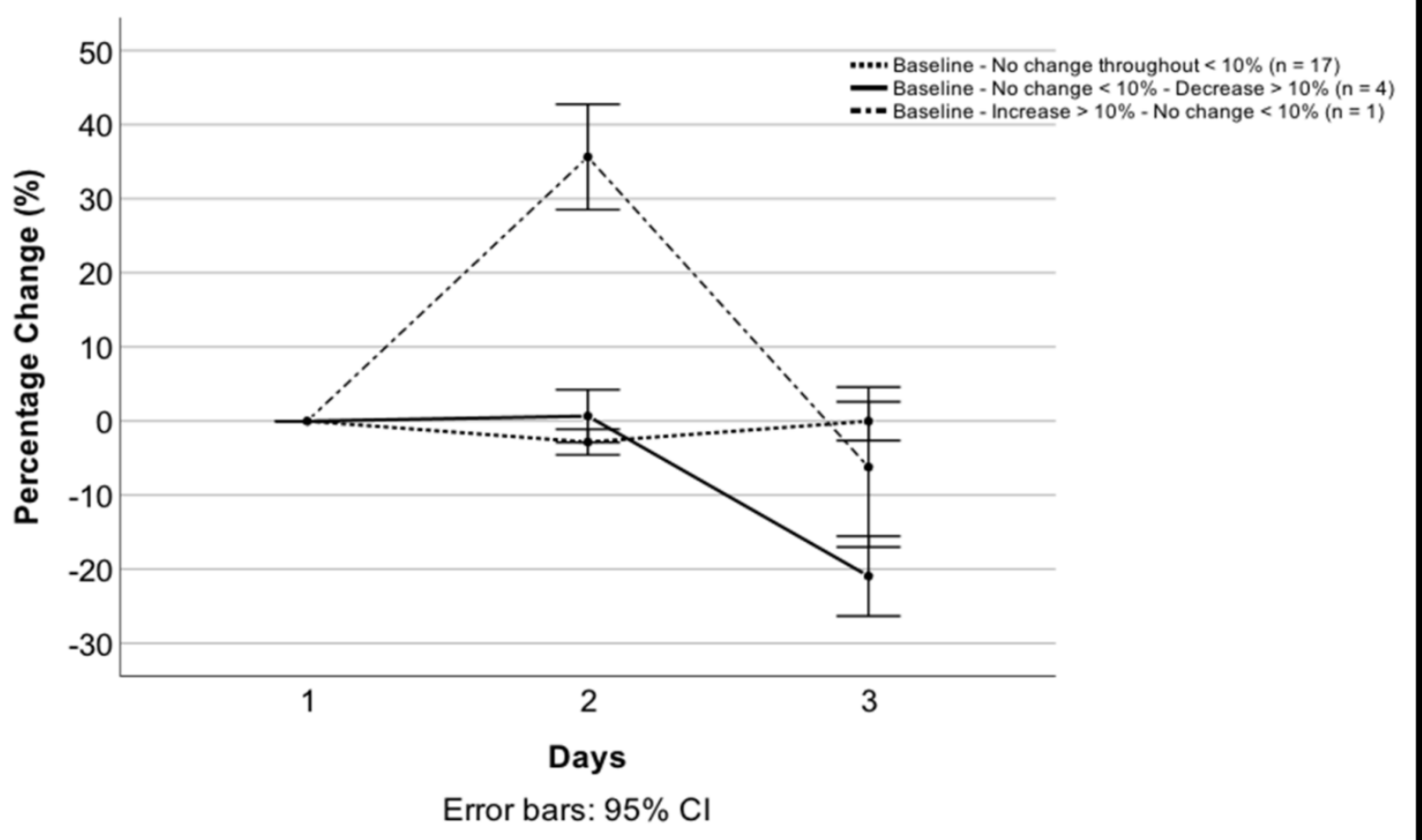

Figure 4.6: Percentage change in Tdi at end-inspiration as compared to the previous day

\subsubsection{Diaphragm thickness (Tdi) at end-expiration}

Similar results were obtained for changes in Tdi during expiration. Eighty-two percent of the participants $(n=18 / 22)$ presented with no change in Tdi on both day two and day three. There was $1 / 22$ participant (4\%) that presented with an increase of more than $10 \%$ in Tdi on day two, but no change in Tdi on day three, and 3/22 (14\%) participants that presented with no change in Tdi on day two, but a decrease of more than $10 \%$ in Tdi on day three (Figure 4.7). The mean (SD) for the Tdi at end-expiration for the three categories between day one and day three are represented in Table 4.7. 
Table 4.7: Tdi at end-expiration for the three categories

\begin{tabular}{|c|c|c|c|}
\hline \multicolumn{3}{|c|}{ Tdi at end-expiration } \\
\hline & Day 1 & Day 2 & Day 3 \\
\hline $\begin{array}{c}\text { Baseline } \\
(\mathrm{n}=22)\end{array}$ & $\begin{array}{c}2.4 \pm 0.6 \\
(\mathrm{~mm})\end{array}$ & & \\
\hline $\begin{array}{c}\text { Group 1 } \\
\text { No chang }<10 \% \\
(\mathrm{n}=18)\end{array}$ & & $\begin{array}{c}2.3 \pm 0.6 \\
(\mathrm{~mm})\end{array}$ & $\begin{array}{c}2.3 \pm 0.6 \\
(\mathrm{~mm})\end{array}$ \\
\hline $\begin{array}{c}\text { Group 2 } \\
(\mathrm{n}=3)\end{array}$ & & $\begin{array}{c}2.2 \pm 0.8 \\
(\mathrm{~mm})\end{array}$ & $\begin{array}{c}1.9 \pm 0.6 \\
(\mathrm{~mm})\end{array}$ \\
\hline $\begin{array}{c}\text { Group 3 } \\
\text { No change }<10 \% \\
(\mathrm{n}=1)\end{array}$ & $\begin{array}{c}2.7 \\
(\mathrm{~mm})\end{array}$ & $\begin{array}{c}2.6 \\
(\mathrm{~mm})\end{array}$ \\
\hline Increase $>10 \%$ & & & \\
\hline
\end{tabular}

Values are represented as mean $\pm \mathrm{SD}$; $\mathrm{mm}$ - millimetres

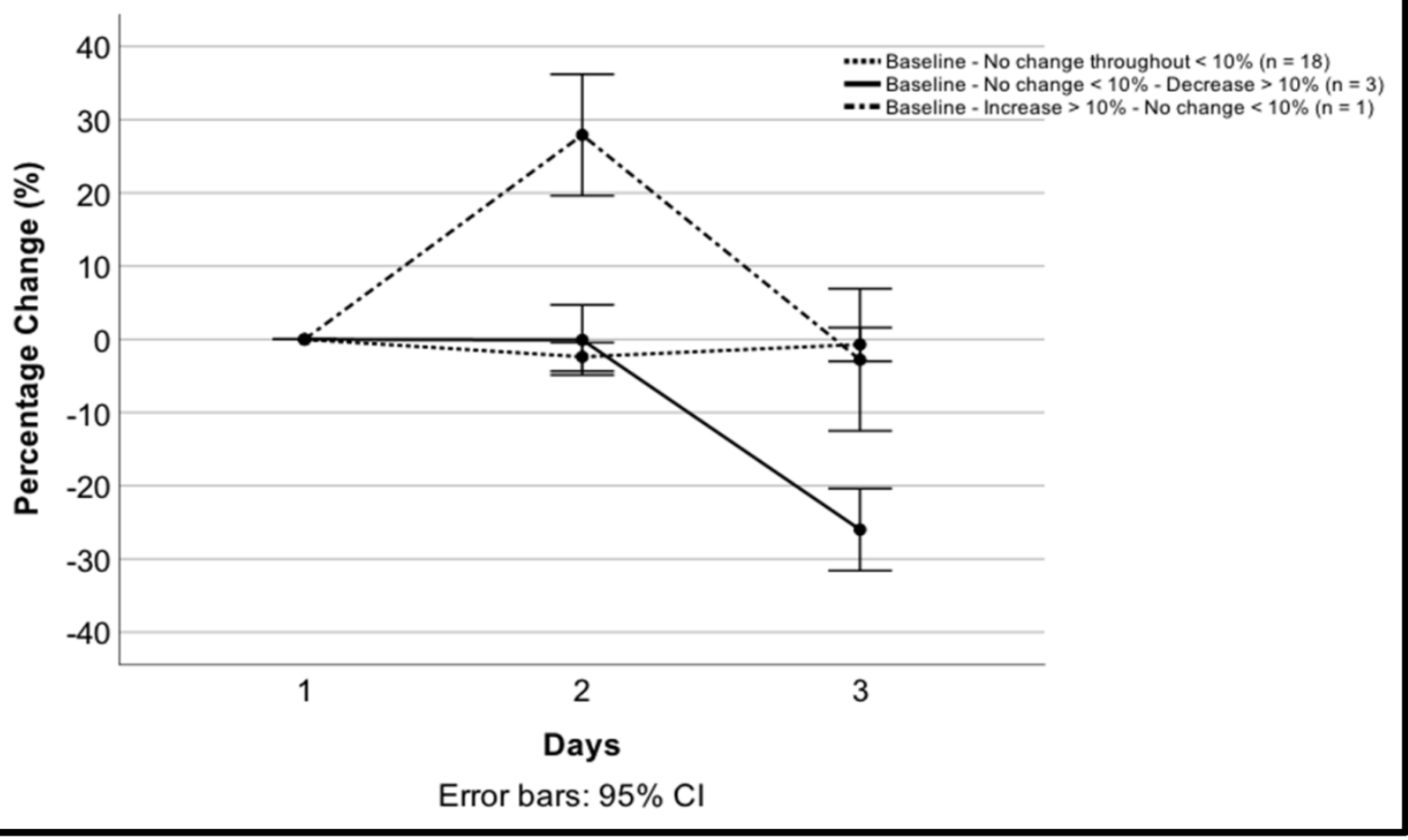

Figure 4.7: Percentage change in Tdi at end-expiration as compared to the previous day 


\subsubsection{Diaphragm Thickening Fraction (DTF)}

The DTF varied over the three days whereby, on day two it decreased to a median (IQR) of 8.7 $(5.6-13.9) \%$ and increased to a mean (SD) of $9.9 \pm 5.3 \%$ on day three (Table 4.8$)$.

Table 4.8: Results of the Diaphragm Thickening Fraction (DTF)

\begin{tabular}{|c|c|c|c|}
\hline Respiratory muscles & $\begin{array}{c}\text { Day 1 } \\
(\mathbf{n = 3 6})\end{array}$ & $\begin{array}{c}\text { Day 2 } \\
(\mathbf{n = 2 5})\end{array}$ & $\begin{array}{c}\text { Day 3 } \\
(\mathbf{n}=\mathbf{2 2})\end{array}$ \\
\hline $\begin{array}{c}\text { Diaphragm Thickening Fraction } \\
\text { (DTF) } \\
\%\end{array}$ & $9.3(6.2-13.7)$ & $8.7(5.6-13.9)$ & $9.9 \pm 5.3$ \\
\hline
\end{tabular}

Values are represented as a mean $\pm \mathrm{SD}$, median (interquartile range); \% - percentage

\subsubsection{Peripheral muscles}

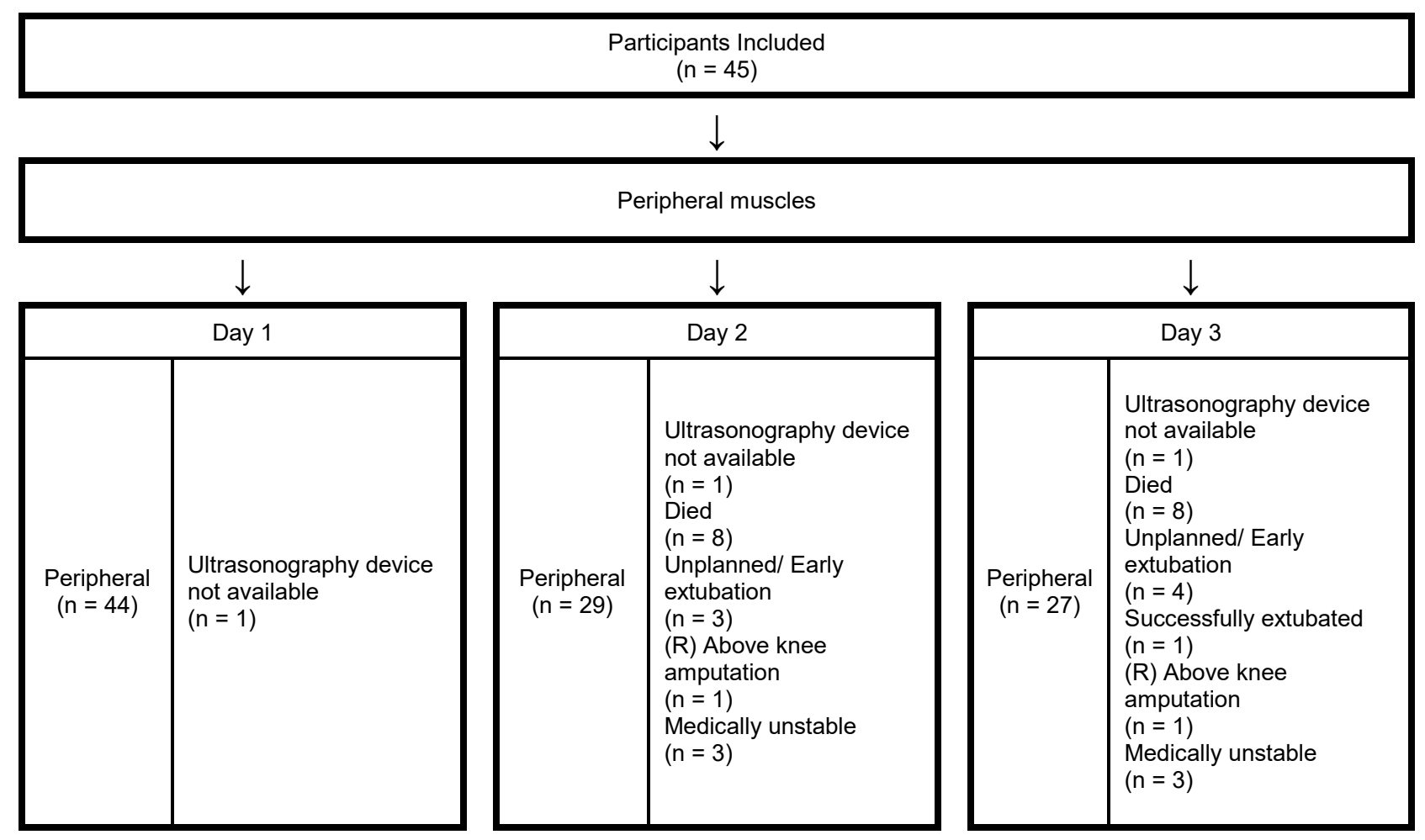

On day one, the mean (SD) for RF muscle thickness was $14.5 \pm 3.7 \mathrm{~mm}$ and the median (IQR) for RF cross-sectional area (CSA) was 219.5 (126.5 - 251.8) $\mathrm{mm}^{2}$, and subsequently declined over the three days (Figure 4.8; Figure 4.9). The median (IQR) on day one for VI muscle echogenicity was 23.7 (13.2 - 44.6) and increased over the three days. The results for both RF and VI muscle thickness and echogenicity are shown in Table 4.9. 
Table 4.9: Ultrasonography of the Peripheral muscles

\begin{tabular}{|c|c|c|c|}
\hline Peripheral muscles & $\begin{array}{c}\text { Day 1 } \\
(\mathbf{n}=\mathbf{4 4})\end{array}$ & $\begin{array}{c}\text { Day 2 } \\
(\mathbf{n}=\mathbf{2 9})\end{array}$ & $\begin{array}{c}\text { Day 3 } \\
(\mathbf{n}=\mathbf{2 7})\end{array}$ \\
\hline $\begin{array}{c}\text { RF Thickness } \\
(\mathrm{mm})\end{array}$ & $14.5 \pm 3.7$ & $13.4 \pm 3.4$ & $13.1 \pm 2.8$ \\
RF CSA & & $207(106-241)$ \\
$\left(\mathrm{mm}^{2}\right)$ & $219.5(126.5-251.8)$ & $215.3(118.5-241.5)$ & $50.1(19.2-59.4)$ \\
RF Echogenicity & $52.5(25.4-60.5)$ & $49.4(24-64.9)$ & $10.6 \pm 2.8$ \\
VI Thickness & $11.9(9.4-15.1)$ & $10.5(8.8-12.5)$ & $36.1(10.2-52.1)$ \\
\hline $\begin{array}{l}(m m) \\
\text { VI Echogenicity }\end{array}$ & $23.7(13.2-44.6)$ & $31.4 \pm 20.5$ & \\
\hline
\end{tabular}

RF - Rectus Femoris; VI - Vastus Intermedius; Values are represented as mean \pm SD, or median (interquartile range); CSA - Cross-Sectional Area; mm - millimetres

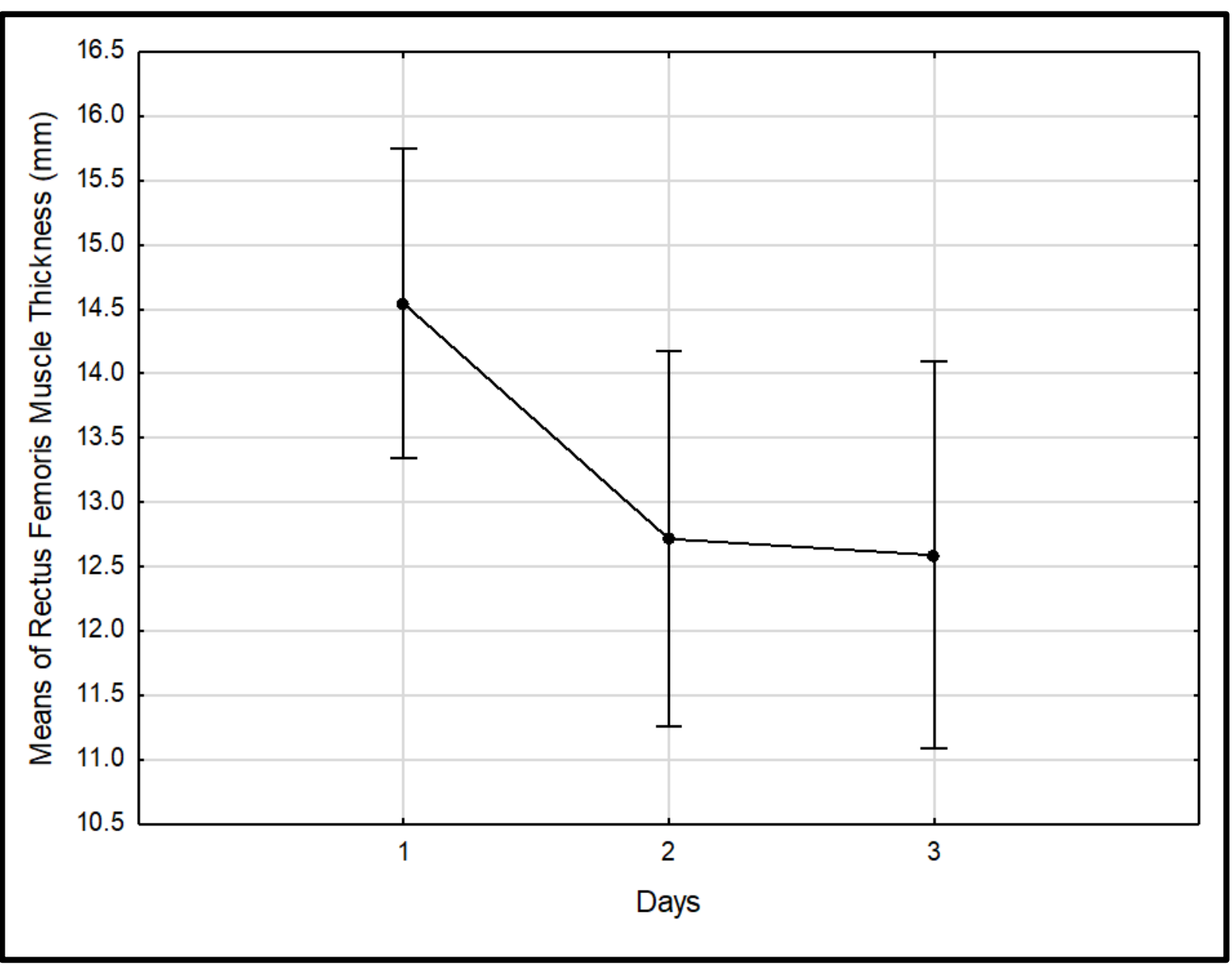

Figure 4.8: Decline in the rectus femoris muscle thickness from day one to day three 


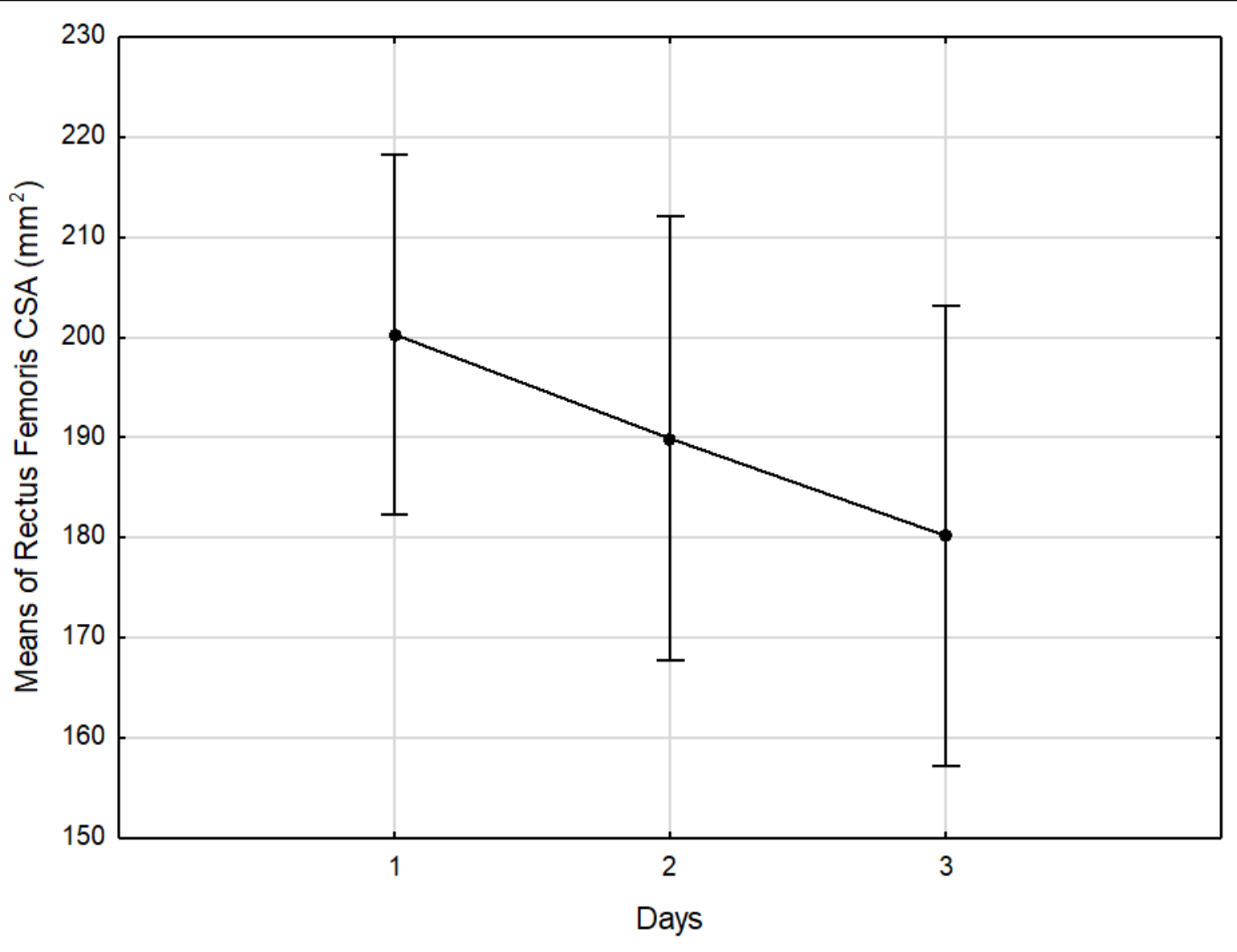

Figure 4.9: Decline in the rectus femoris muscle CSA from day one to day three

\subsection{Peripheral muscle strength}

\subsubsection{Medical Research Council - Sum Score (MRC-SS)}

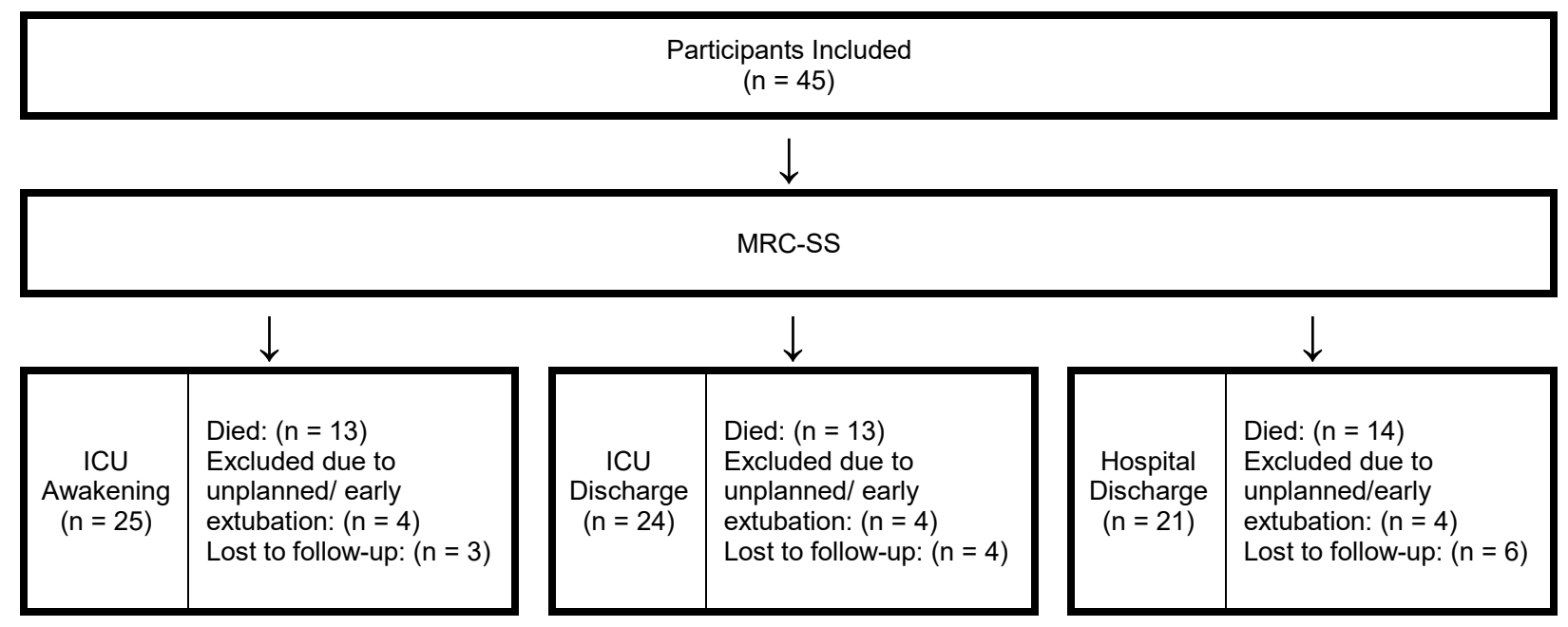


The median (IQR) MRC-SS at ICU awakening was 48 (44 - 51), increased to a median (IQR) MRC-SS of 52 (45 - 54) at ICU discharge and a median (IQR) MRC-SS of 54 (47 - 56) at hospital discharge. Furthermore, the minimum and maximum MRC-SS at ICU awakening, at ICU discharge and at hospital discharge were: 31 and 58 points, 31 and 60 points, and 36 and 60 points respectively. The number of participants that scored 60 on the MRC-SS at ICU discharge and at hospital discharge are represented in Figure 4.10. Figure 4.11 represents the percentage and number of participants that presented with ICU-AW at the three-time intervals and also indicating a decline in the percentage of participants at ICU discharge and hospital discharge.

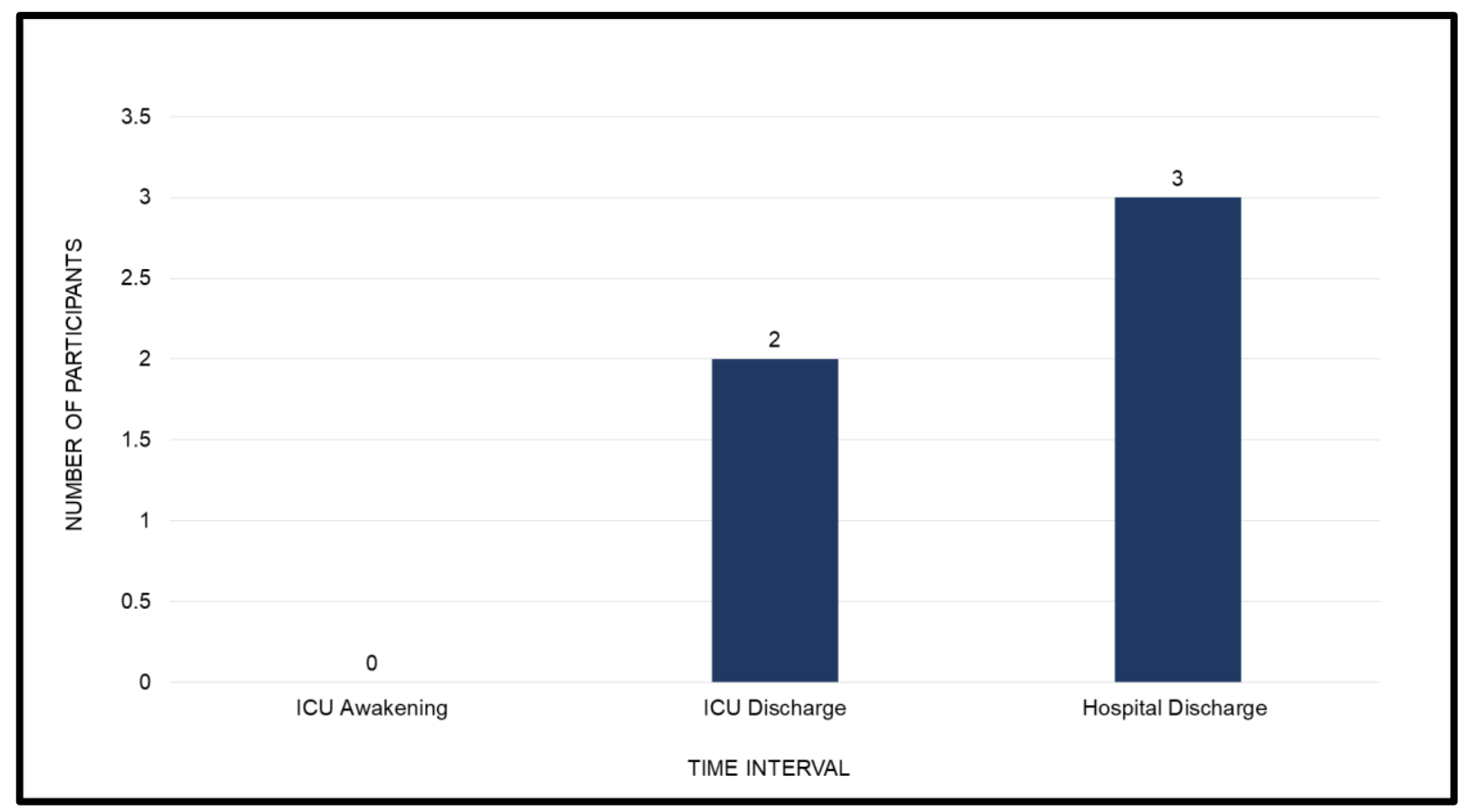

Figure 4.10: Number of participants that scored 60 on the MRC-SS 


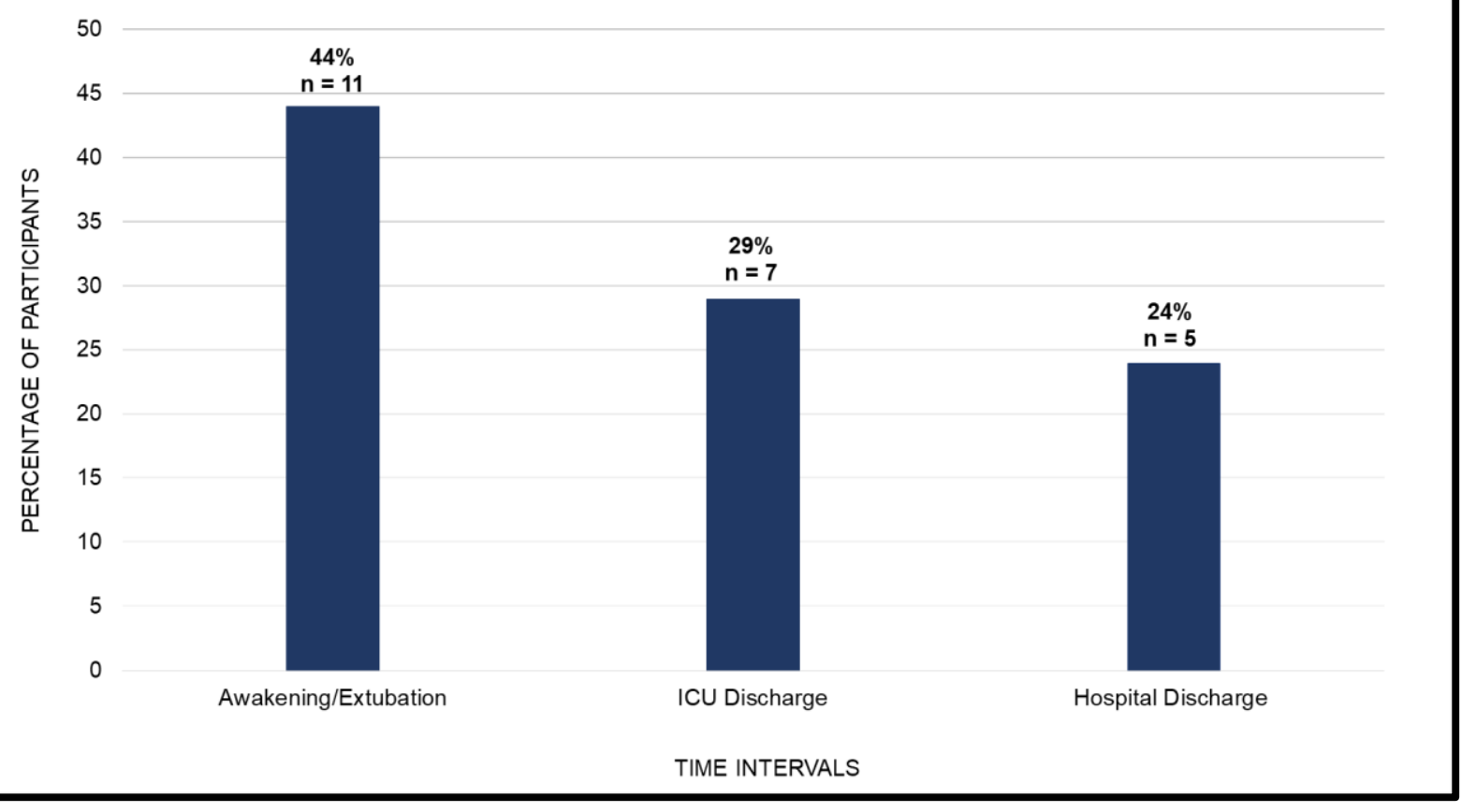

Figure 4.11: Percentage of participants with ICU-AW (MRC-SS $<48$ ) at the three-time intervals

\subsubsection{Dynamometry}

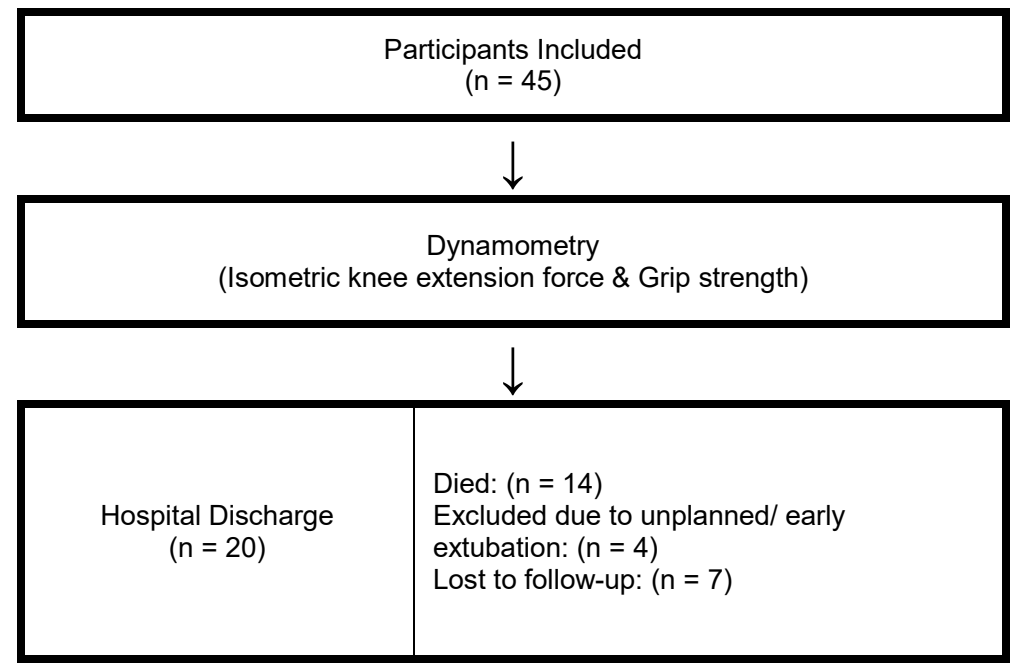

The minimum and maximum hand-grip strength scores were 10 and $50 \mathrm{~N}$ for the dominant upper limb and 8 and $50 \mathrm{~N}$ for the non-dominant upper limb. The mean (SD) isometric knee extension force of the dominant lower limb was $96.8 \pm 25 \mathrm{~N}$ and the median (IQR) isometric knee extension force of the non-dominant lower limb was $78.4(61.4-92.2)$ N. Furthermore, participants scored a mean (SD) of $22.2 \pm 5.1 \%$ of the expected isometric knee extension force for the dominant lower limb and a median (IQR) of 17 (13 - 23.7) \% of the expected isometric knee extension force for the 
non-dominant lower limb. The results for hand-grip strength and isometric knee extension force of both the dominant and non-dominant upper and lower limbs are represented in Table 4.10. The normative values for hand-grip strength and isometric knee extension force are represented in Table 4.11 and Table 4.12.

Table 4.10: Results of Dynamometry

\begin{tabular}{|c|c|c|}
\hline \multicolumn{3}{|c|}{ Dynamometry } \\
\hline & Mean \pm SD / Median (IQR) & Min - Max \\
\hline Hand-grip Strength $(\mathrm{Kg})$ & & \\
\hline $\begin{array}{c}\text { Dominant Upper Limb } \\
\qquad(\mathrm{n}=20)\end{array}$ & $26.9 \pm 11.5$ & $10-50$ \\
\hline $\begin{array}{l}\text { Non-dominant Upper Limb } \\
\qquad(\mathrm{n}=20)\end{array}$ & $26.4 \pm 10.2$ & $8-50$ \\
\hline Isometric Knee Extension Force (N & & \\
\hline $\begin{array}{l}\text { Dominant Lower Limb } \\
\qquad(\mathrm{n}=20)\end{array}$ & $96.8 \pm 25$ & $60-146$ \\
\hline $\begin{array}{l}\text { Expected Knee Extension Force } \\
\qquad(n=18)\end{array}$ & $446.5 \pm 73$ & \\
\hline $\begin{array}{l}\text { Percentage of Predicted } \\
\qquad(n=18)\end{array}$ & $22.2 \pm 5.1$ & \\
\hline $\begin{array}{l}\text { Non-dominant Lower Limb } \\
\qquad(\mathrm{n}=20)\end{array}$ & $78.4(61.9-92.2)$ & $0-139$ \\
\hline $\begin{array}{l}\text { Expected Knee Extension Force } \\
\qquad(n=18)\end{array}$ & $444.9 \pm 70.7$ & \\
\hline $\begin{array}{l}\text { Percentage of Predicted } \\
\qquad(n=18)\end{array}$ & $17(13-23.7)$ & \\
\hline
\end{tabular}

Values are represented as mean $\pm \mathrm{SD}$, median (interquartile range) or minimum and maximum; $\mathrm{Kg}-\mathrm{Kilograms;} \mathrm{N}$ - Newtons 
Table 4.11: Normative values for Hand-grip Strength

\begin{tabular}{|c|c|c|c|}
\hline Age & Gender & $\begin{array}{l}\text { Right Upper Limb/ } \\
\text { Left Upper Limb }\end{array}$ & Hand-grip Strength $(\mathrm{Kg})$ \\
\hline \multirow{4}{*}{$20-24$} & \multirow{2}{*}{ M } & $\mathrm{R}$ & $52.3 \pm 9.8$ \\
\hline & & L & $50.1 \pm 8.4$ \\
\hline & \multirow{2}{*}{$\mathrm{F}$} & $\mathrm{R}$ & $31.8 \pm 4.6$ \\
\hline & & L & $30.7 \pm 4.6$ \\
\hline \multirow{4}{*}{$25-29$} & \multirow{2}{*}{ M } & $\mathrm{R}$ & $52.7 \pm 9.3$ \\
\hline & & L & $51.8 \pm 8.7$ \\
\hline & \multirow{2}{*}{$\mathrm{F}$} & $\mathrm{R}$ & $34.1 \pm 6.0$ \\
\hline & & L & $33.1 \pm 5.7$ \\
\hline \multirow{4}{*}{$30-34$} & \multirow{2}{*}{ M } & $\mathrm{R}$ & $52.6 \pm 9.7$ \\
\hline & & L & $51.0 \pm 8.6$ \\
\hline & \multirow{2}{*}{$\mathrm{F}$} & $\mathrm{R}$ & $34.9 \pm 6.1$ \\
\hline & & L & $33.4 \pm 5.8$ \\
\hline \multirow{4}{*}{$34-39$} & \multirow{2}{*}{ M } & $\mathrm{R}$ & $52.5 \pm 8.8$ \\
\hline & & $\mathrm{L}$ & $51.1 \pm 8.4$ \\
\hline & \multirow{2}{*}{$\mathrm{F}$} & $\mathrm{R}$ & $37.3 \pm 6.2$ \\
\hline & & $\mathrm{L}$ & $35.6 \pm 6.2$ \\
\hline \multirow{4}{*}{$40-44$} & \multirow{2}{*}{ M } & $\mathrm{R}$ & $52.0 \pm 9.1$ \\
\hline & & L & $50.9 \pm 9.9$ \\
\hline & \multirow{2}{*}{$\mathrm{F}$} & $\mathrm{R}$ & $35.7 \pm 6.8$ \\
\hline & & L & $34.0 \pm 8.0$ \\
\hline \multirow{4}{*}{$44-49$} & \multirow{2}{*}{ M } & $\mathrm{R}$ & $49.6 \pm 11.0$ \\
\hline & & $\mathrm{L}$ & $49.9 \pm 11.6$ \\
\hline & \multirow{2}{*}{$\mathrm{F}$} & $\mathrm{R}$ & $34.5 \pm 4.6$ \\
\hline & & L & $32.6 \pm 4.6$ \\
\hline \multirow{4}{*}{$50-55$} & \multirow{2}{*}{ M } & $\mathrm{R}$ & $45.1 \pm 9.4$ \\
\hline & & $\mathrm{L}$ & $43.9 \pm 8.9$ \\
\hline & \multirow{2}{*}{$\mathrm{F}$} & $\mathrm{R}$ & $35.3 \pm 5.4$ \\
\hline & & L & $33.2 \pm 4.6$ \\
\hline
\end{tabular}

M - Male; F - Female; R - Right; L - Left; Kg - Kilograms

Table 4.12: Normative values for Isometric Knee Extension Force

\begin{tabular}{|c|c|c|}
\hline Age & $\begin{array}{c}\text { Dominant Lower Limb / } \\
\text { Non-dominant Lower Limb }\end{array}$ & $\begin{array}{c}\text { Isometric Knee } \\
\text { Extension Force (N) }\end{array}$ \\
\hline \multirow{2}{*}{$20-29$} & Dominant & $575.2 \pm 92.3$ \\
Non - dominant & $578.6 \pm 94.7$ \\
\hline \multirow{2}{*}{$30-39$} & Dominant & $572.9 \pm 76.5$ \\
& Non - dominant & $572.5 \pm 82.8$ \\
\hline \multirow{2}{*}{$40-49$} & Dominant & $583 \pm 73.7$ \\
\hline \multirow{2}{*}{$50-59$} & Non - dominant & $588.9 \pm 72.5$ \\
\hline \multirow{2}{*}{$60-69$} & Dominant & $470.9 \pm 92.3$ \\
& Non - dominant & $467.7 \pm 103.1$ \\
\hline & Dominant & $386.9 \pm 94.3$ \\
& Non - dominant & $376.5 \pm 67.3$ \\
\hline
\end{tabular}




\subsection{Respiratory muscle strength and endurance}

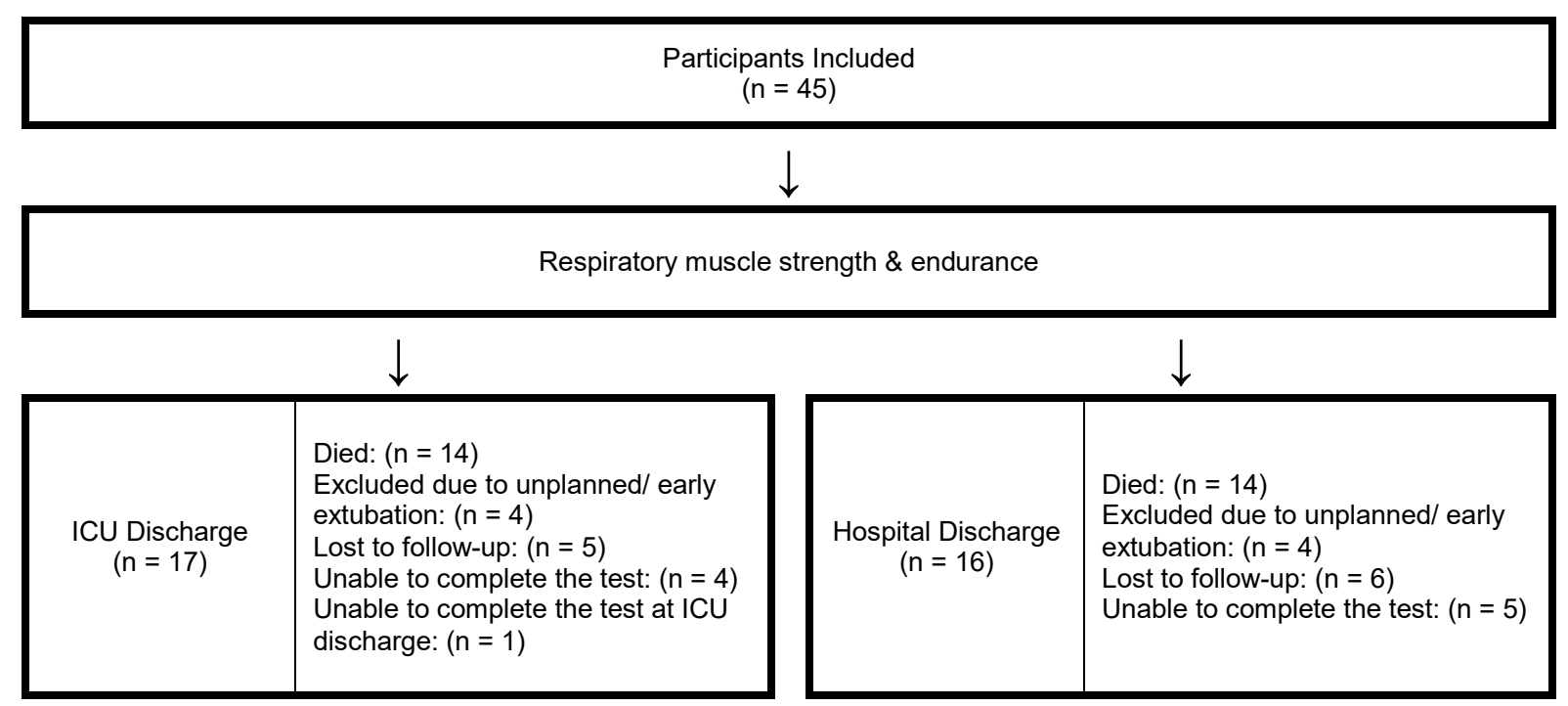

The results of respiratory muscle strength at ICU discharge and hospital discharge are represented in Table 4.13. The results for respiratory muscle endurance are as follows: the median (IQR) time for the completion of the test was $2(1.5-2.8)$ minutes, with a mean (SD) load of $15.7 \pm 5.5$ joules and a median (IQR) power of $0.8(0.4-1)$ watts (Table 4.13). The normative values for respiratory muscle strength are represented in Table 4.14.

Table 4.13: Results of Respiratory muscle strength and endurance

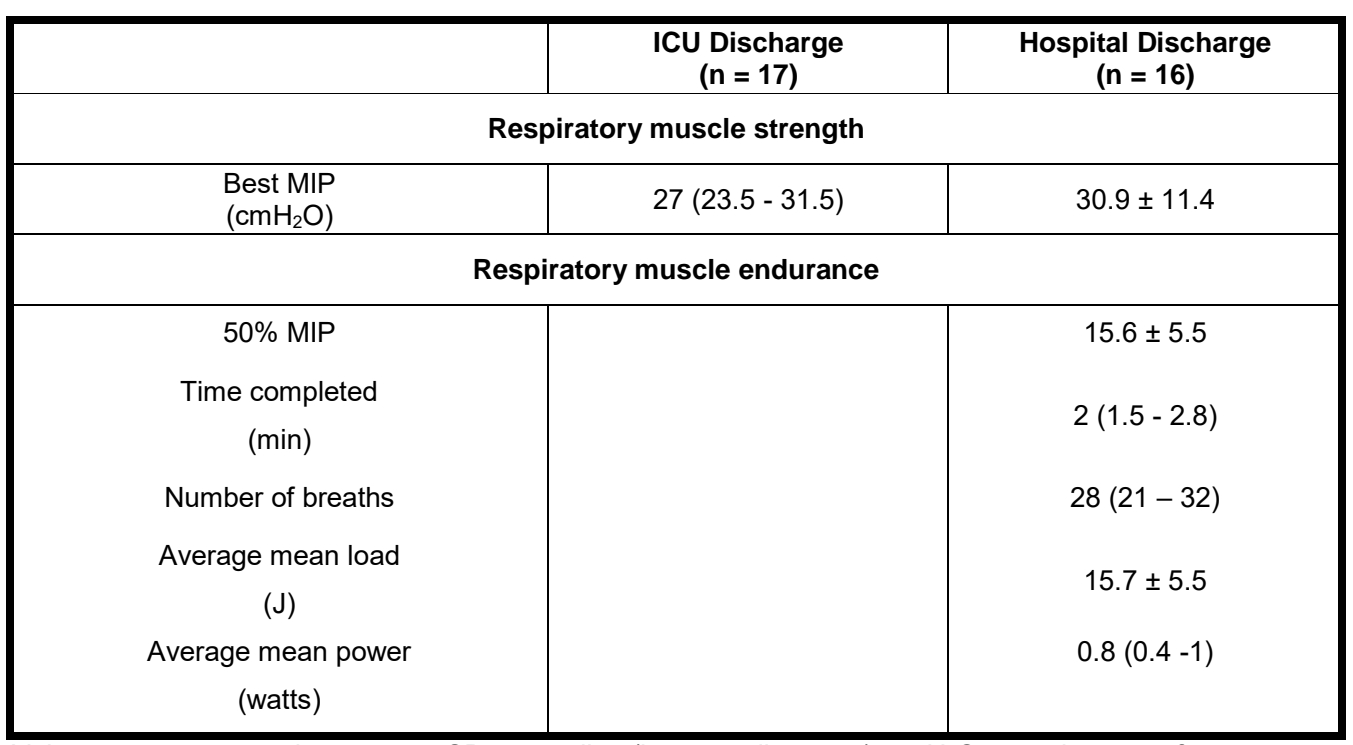

Values are represented as mean $\pm \mathrm{SD}$, or median (interquartile range); $\mathrm{cmH}_{2} \mathrm{O}$ - centimetres of water; min - minutes; $\mathrm{J}$ - Joules 
Table 4.14: Normative values for Respiratory muscle strength

\begin{tabular}{|c|c|c|}
\hline Age & Gender & MIP $\left(\mathbf{c m H}_{2} \mathbf{O}\right)$ \\
\hline \multirow{2}{*}{$20-29$} & $\mathrm{M}$ & $127.0 \pm 22.9$ \\
& $\mathrm{~F}$ & $100.5 \pm 12.1$ \\
\hline \multirow{2}{*}{$30-39$} & $\mathrm{M}$ & $127.2 \pm 21.7$ \\
& $\mathrm{~F}$ & $94.5 \pm 12.1$ \\
\hline \multirow{2}{*}{$40-49$} & $\mathrm{M}$ & $124.3 \pm 6.0$ \\
& $\mathrm{~F}$ & $107.5 \pm 6.3$ \\
\hline \multirow{2}{*}{$50-59$} & $\mathrm{M}$ & $115.0 \pm 4.0$ \\
& $\mathrm{~F}$ & $85.0 \pm 4.0$ \\
\hline \multirow{2}{*}{$60-69$} & $\mathrm{M}$ & $89.5 \pm 4.3$ \\
& $\mathrm{~F}$ & $73.0 \pm 6.7$ \\
\hline
\end{tabular}

$\mathrm{M}$ - Male; F - Female; $\mathrm{cmH}_{2} \mathrm{O}$ - centimetres of water

\subsection{Physical function}

\subsubsection{The Chelsea Critical Care Assessment (CPAx) tool and the de Morton Mobility Index} (DEMMI)

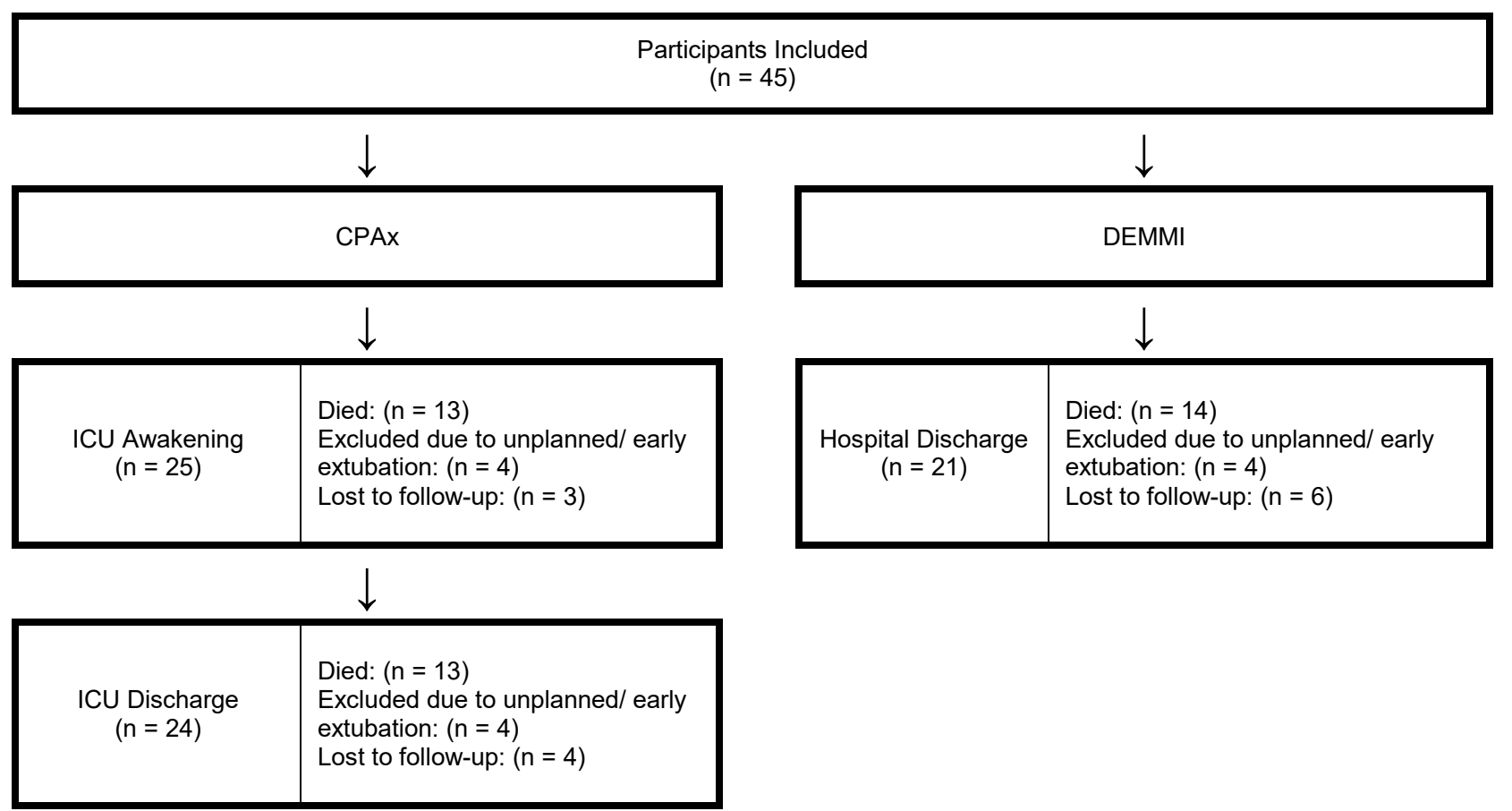

Participants presented with reduced physical function at ICU awakening, ICU discharge and at hospital discharge. The CPAx score increased by 2.5 points at ICU discharge when compared to the CPAx score at ICU awakening. The results for physical function are represented in Table 4.15. 
Table 4.15: Results of CPAx and DEMMI

\begin{tabular}{|c|c|c|c|}
\hline & $\begin{array}{c}\text { ICU Awakening } \\
(\mathbf{n = 2 5})\end{array}$ & $\begin{array}{c}\text { ICU Discharge } \\
(\mathbf{n = 2 4 )}\end{array}$ & $\begin{array}{c}\text { Hospital Discharge } \\
(\mathbf{n}=\mathbf{2 1})\end{array}$ \\
\hline CPAx & $33(29-34)$ & $35.5(33.3-39)$ & $13.7(10.5-17)$ \\
DEMMI Raw Score & & & $60.9 \pm 21.9$ \\
\hline
\end{tabular}

Values are represented as mean $\pm \mathrm{SD}$, or median (interquartile range)

\subsubsection{Six-Minute Walk Test (6-MWT)}

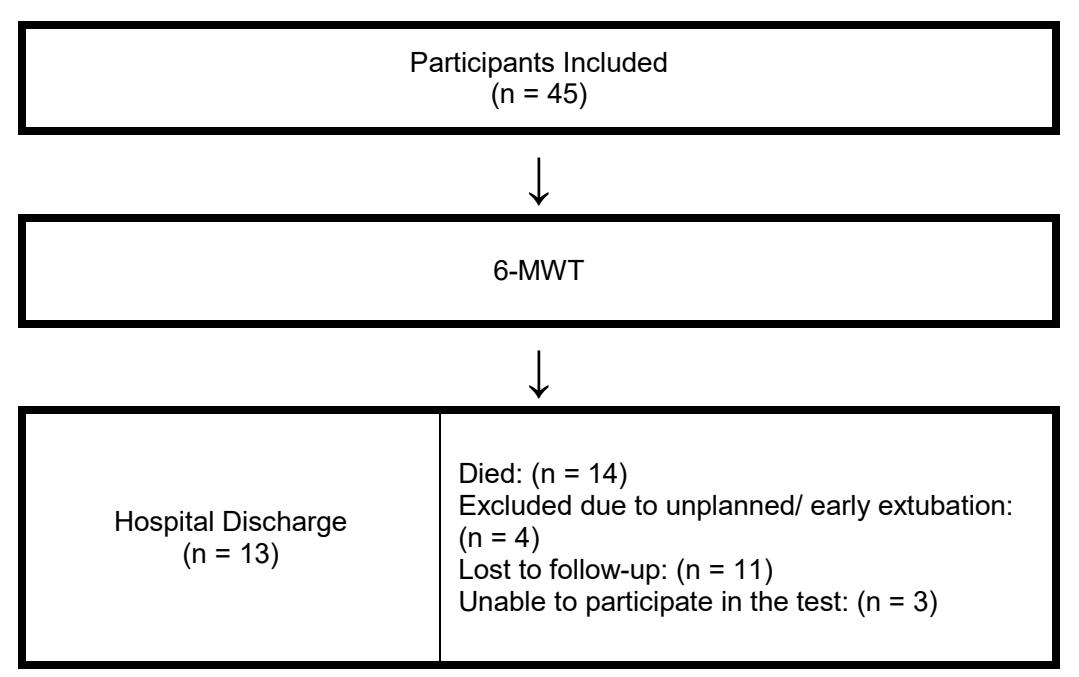

Three participants were unable to participate in the 6-MWT due to the following reasons: 1) physiologically unstable participants; and 2) a vacuum-assisted closure (VAC) limiting participant to complete the test. Twelve participants (92.3\%) completed two 6-MWTs whereas one participant only completed one 6-MWT. The reason for the one participant not being able to complete a second 6-MWT was due to tiredness. None of the participants used oxygen or a walking aid when completing the 6-MWTs. Table 4.16 represents the results for the first, second and the best 6 MWT distance. The normative values for the 6-MWT are represented in Table 4.17. 
Table 4.16: Results of the 6-MWT

\begin{tabular}{|c|c|c|}
\hline \multicolumn{3}{|c|}{ Six-Minute Walk Test (6-MWT) } \\
\hline & Mean \pm SD & Min - Max \\
\hline $\begin{array}{l}\text { 6-MWT-1 Distance }(m) \\
(n=13)\end{array}$ & $338.5 \pm 111.5$ & $120-502$ \\
\hline $\begin{array}{c}\text { 6-MWT-2 Distance }(m) \\
\qquad(\mathrm{n}=12)\end{array}$ & $349.3 \pm 87.2$ & $220-499$ \\
\hline $\begin{array}{l}\text { Best 6-MWT Distance }(m) \\
\qquad(n=13)\end{array}$ & $352.8 \pm 111.7$ & $120-502$ \\
\hline
\end{tabular}

Values are represented as mean $\pm \mathrm{SD}$, or minimum and maximum; $\mathrm{m}-$ metres

Table 4.17: Normative values for the 6-MWT

\begin{tabular}{|c|c|c|}
\hline Age & Gender & 6 MWD \\
\hline $21-70$ & $\mathrm{M}$ & $711 \pm 81(\mathrm{~m})$ \\
& $\mathrm{F}$ & $511 \pm 75(\mathrm{~m})$ \\
\hline
\end{tabular}

M - Male; F - Female; $m$ - metres

\subsection{Health-related quality of life (HRQoL)}

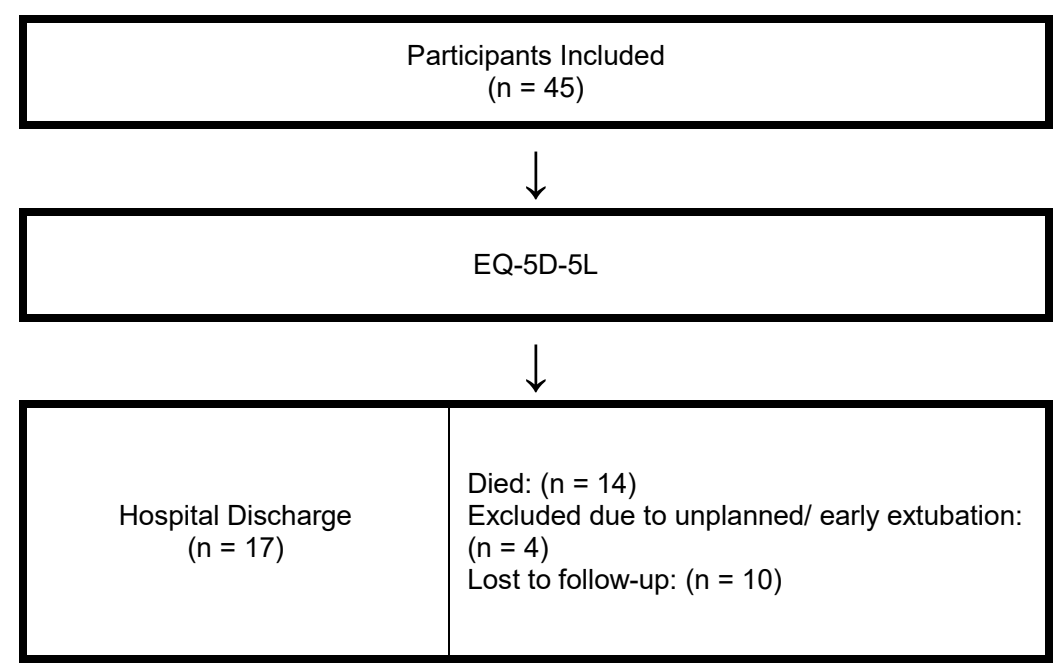

\subsubsection{EQ-5D-5L}

The number of participants that scored for each category of the five dimensions of health are represented in Figure 4.12. There were participants who presented with moderate problems to being unable to complete tasks for the dimensions of mobility, self-care and usual activities, and participants who presented with moderate to extreme pain or discomfort. Only one participant indicated being severely depressed or anxious at the time of hospital discharge. The mean (SD) VAS score was $76.1 \pm 18.7$, with a minimum score of 40 points and a maximum score of 100 points. 

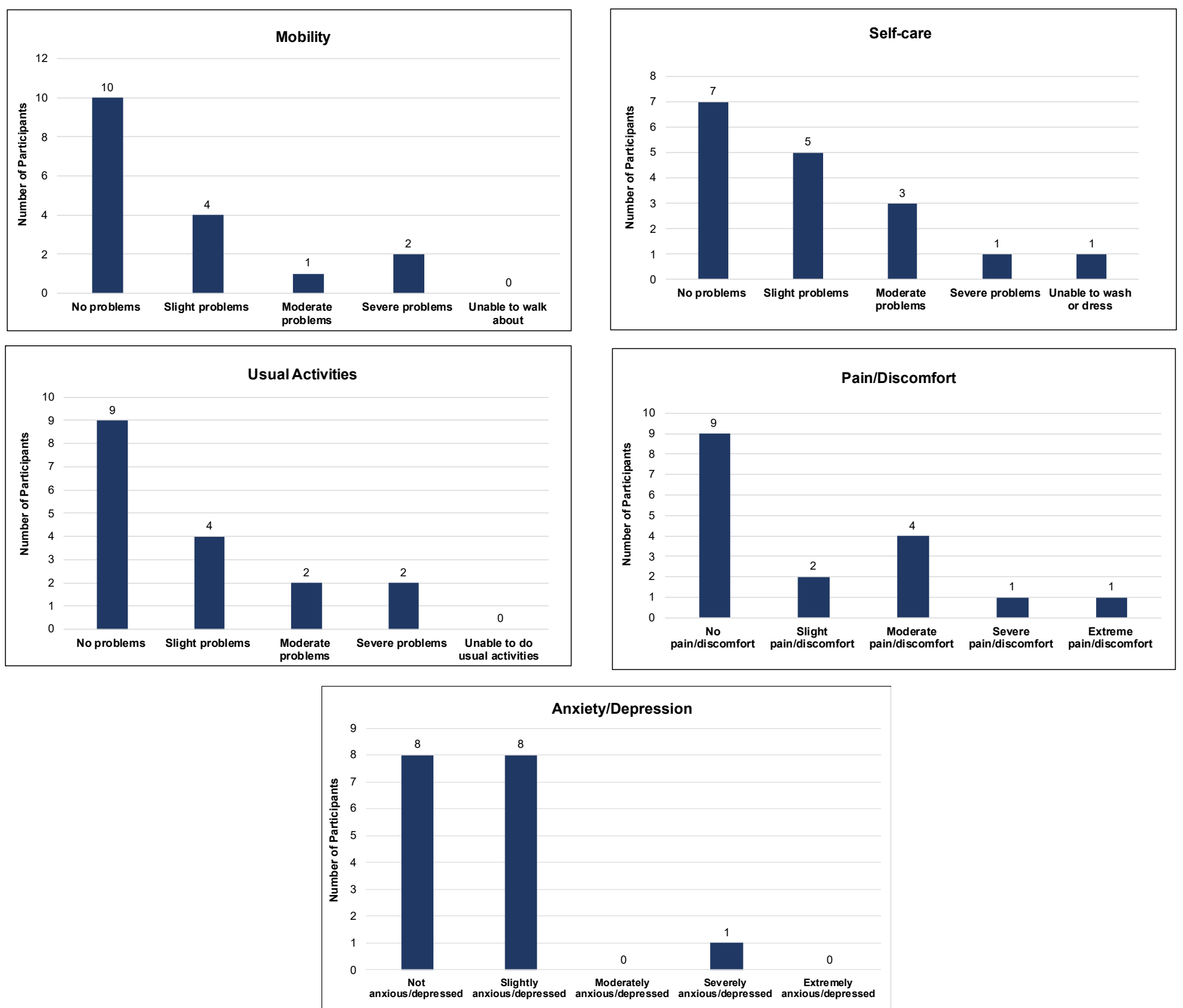

Figure 4.12 : Number of participants that scored for each category of the five dimensions of health as measured with the EQ-5D-5L 


\subsection{Correlation between muscle structure, muscle function and physical function}

\subsubsection{ICU awakening}

The correlations between muscle structure, muscle function or physical function were very weak or weak non-significant correlations at ICU awakening. Whether these correlations were found by chance will need to be investigated in future studies. The results for the correlations between muscle structure, muscle function and physical function are represented in Table 4.18.

Table 4.18: Correlations between muscle structure, muscle function, and physical function at ICU awakening

\begin{tabular}{|c|c|c|c|}
\hline Parameter & Comparator & \multicolumn{2}{|c|}{ ICU Awakening } \\
\hline & & rho & $P$ \\
\hline $\begin{array}{c}\text { MRC-SS } \\
\text { (Awakening/Extubation) }\end{array}$ & $\begin{array}{c}\text { CPAx } \\
\text { (Awakening/Extubation) } \\
(\mathrm{n}=25)\end{array}$ & 0.15 & 0.47 \\
\hline DTF & $\begin{array}{c}\text { CPAx } \\
\text { (Awakening/Extubation) } \\
(\mathrm{n}=17)\end{array}$ & 0.17 & 0.51 \\
\hline Tdi During End-Expiration & $\begin{array}{c}\text { CPAx } \\
\text { (Awakening/Extubation) } \\
(\mathrm{n}=17)\end{array}$ & -0.12 & 0.64 \\
\hline \multirow{2}{*}{ RF Muscle Thickness } & $\begin{array}{c}\text { MRC-SS } \\
\text { (Awakening/Extubation) } \\
(\mathrm{n}=22)\end{array}$ & 0.32 & 0.14 \\
\hline & $\begin{array}{c}\text { CPAx } \\
\text { (Awakening/Extubation) } \\
(\mathrm{n}=22)\end{array}$ & -0.04 & 0.86 \\
\hline \multirow{2}{*}{$\begin{array}{l}\text { RF Cross-Sectional Area } \\
\text { (CSA) }\end{array}$} & $\begin{array}{c}\text { MRC-SS } \\
\text { (Awakening/Extubation) } \\
(\mathrm{n}=24)\end{array}$ & -0.09 & 0.68 \\
\hline & $\begin{array}{c}\text { CPAx } \\
\text { (Awakening/Extubation) } \\
(\mathrm{n}=24)\end{array}$ & -0.15 & 0.50 \\
\hline \multirow{2}{*}{ RF Muscle Echogenicity } & $\begin{array}{c}\text { MRC-SS } \\
\text { (Awakening/Extubation) } \\
(\mathrm{n}=22)\end{array}$ & -0.26 & 0.25 \\
\hline & $\begin{array}{c}\text { CPAx } \\
\text { (Awakening/Extubation) } \\
(\mathrm{n}=22)\end{array}$ & -0.15 & 0.52 \\
\hline \multirow{2}{*}{ VI Muscle Thickness } & $\begin{array}{c}\text { MRC-SS } \\
\text { (Awakening/Extubation) } \\
(\mathrm{n}=22)\end{array}$ & 0.42 & 0.06 \\
\hline & $\begin{array}{c}\text { CPAx } \\
\text { (Awakening/Extubation) } \\
(\mathrm{n}=22)\end{array}$ & 0.06 & 0.79 \\
\hline \multirow{2}{*}{ VI Muscle Echogenicity } & $\begin{array}{c}\text { MRC-SS } \\
\text { (Awakening/Extubation) } \\
(\mathrm{n}=22)\end{array}$ & -0.39 & 0.07 \\
\hline & $\begin{array}{c}\text { CPAx } \\
\text { (Awakening/Extubation) } \\
(\mathrm{n}=22)\end{array}$ & -0.38 & 0.08 \\
\hline
\end{tabular}

MRC-SS - Medical Research Council - Sum Score; CPAx - Chelsea Critical Care Physical Assessment tool; DTF - Diaphragm Thickening Fraction; Tdi - Diaphragm thickness; RF - Rectus Femoris; VI - Vastus Intermedius; CSA - Cross-Sectional Area

Spearman correlation coefficient was used to determine correlations

*Significant results, $\mathrm{P}<0.05$ 


\subsubsection{ICU discharge}

At ICU discharge, there were only very weak non-significant correlations between muscle function (strength) and physical function. Whether these correlations were found by chance, will need to be investigated in future studies (Table 4.19).

Table 4.19: Correlations between muscle structure, muscle function, and physical function at ICU discharge

\begin{tabular}{|c|c|c|c|}
\hline Parameter & Comparator & \multicolumn{2}{|c|}{ ICU Discharge } \\
\hline & & rho & $P$ \\
\hline $\begin{array}{c}\text { MRC-SS } \\
\text { (ICU Discharge) }\end{array}$ & $\begin{array}{c}\text { CPAx } \\
\text { (ICU Discharge }) \\
(\mathrm{n}=24)\end{array}$ & 0.29 & 0.17 \\
\hline $\begin{array}{c}\text { CPAx } \\
\text { MIP } \\
\text { (ICU Discharge })\end{array}$ & $\begin{array}{c}\text { Discharge }) \\
(\mathrm{n}=17)\end{array}$ & 0.27 & 0.29 \\
\hline
\end{tabular}

MRC-SS - Medical Research Council - Sum Score; CPAx - Chelsea Critical Care Physical Assessment tool; MIP -

Maximum Inspiratory Pressure

Spearman correlation coefficient was used to determine correlations

${ }^{*}$ Significant results, $\mathrm{P}<0.05$

\subsubsection{Hospital discharge}

At hospital discharge, there was a moderate positive correlation between the MRC-SS and functional outcomes as measured by the DEMMI and the 6-MWT (rho $=0.59, P=0.01$; rho $=0.57, P=0.04)$. A moderate positive correlation was also found between the MRC-SS and grip strength of both the dominant and non-dominant upper $\operatorname{limb}(r h o=0.68, P=0.01$; rho $=$ $0.69, P=0.01)$. Similarly, there was a moderate positive correlation between the isometric knee extension force of the non-dominant lower limb and the DEMMI (rho $=0.68 ; P=0.01)$. A strong positive correlation was found between MIP and the 6-MWT ( $r h o=0.75 ; P=0.01$ ). The results also highlight the discrepency between self reported measures and empiric measure of physical function. There was a very weak correlation between participants' perception of health (EQ-5D-5L) and the DEMMI, and a weak inverse correlation between particpants' perception of health (EQ-5D-5L) with the six-minute walk distance (6-MWD) (Table 4.20). 
Table 4.20: Correlations between muscle structure, muscle function, and physical function at hospital discharge

\begin{tabular}{|c|c|c|c|}
\hline Parameter & Comparator & \multicolumn{2}{|c|}{ Hospital Discharge } \\
\hline & & rho & $P$ \\
\hline \multirow{2}{*}{$\begin{array}{c}\text { MRC-SS } \\
\text { (Hospital Discharge) }\end{array}$} & $\begin{array}{l}\text { DEMMI } \\
(\mathrm{n}=21)\end{array}$ & 0.59 & $0.01^{*}$ \\
\hline & $\begin{array}{l}\text { 6-MWT } \\
(n=13)\end{array}$ & 0.57 & $0.04^{*}$ \\
\hline \multirow{4}{*}{$\begin{array}{c}\text { MRC-SS } \\
\text { (Hospital Discharge) }\end{array}$} & $\begin{array}{c}\text { Grip Strength } \\
\text { (Dominant Upper Limb) } \\
(\mathrm{n}=20) \\
\end{array}$ & 0.68 & $0.01^{*}$ \\
\hline & $\begin{array}{c}\text { Grip Strength } \\
\text { (Non - Dominant Upper Limb) } \\
(\mathrm{n}=20)\end{array}$ & 0.69 & $0.01^{*}$ \\
\hline & $\begin{array}{l}\text { Knee Extension Force } \\
\text { (Dominant Lower Limb) } \\
(\mathrm{n}=20)\end{array}$ & 0.08 & 0.72 \\
\hline & $\begin{array}{c}\text { Knee Extension Force } \\
\text { (Non - Dominant Lower Limb) } \\
(\mathrm{n}=20)\end{array}$ & 0.29 & 0.22 \\
\hline \multirow{2}{*}{$\begin{array}{l}\text { Knee Extension Force } \\
\text { (Dominant Lower Limb) }\end{array}$} & $\begin{array}{l}\text { DEMMI } \\
(n=20)\end{array}$ & 0.30 & 0.21 \\
\hline & $\begin{array}{l}\text { 6-MWT } \\
(\mathrm{n}=13)\end{array}$ & 0.28 & 0.36 \\
\hline \multirow{2}{*}{$\begin{array}{c}\text { Knee Extension Force } \\
\text { (Non - Dominant Lower Limb) }\end{array}$} & $\begin{array}{l}\text { DEMMI } \\
(n=20)\end{array}$ & 0.68 & $0.01^{*}$ \\
\hline & $\begin{array}{l}\text { 6-MWT } \\
(n=13)\end{array}$ & 0.42 & 0.16 \\
\hline \multirow{2}{*}{$\begin{array}{c}\text { Grip Strength } \\
\text { (Dominant Upper Limb) }\end{array}$} & $\begin{array}{l}\text { DEMMI } \\
(\mathrm{n}=20)\end{array}$ & 0.28 & 0.22 \\
\hline & $\begin{array}{l}\text { 6-MWT } \\
(\mathrm{n}=13)\end{array}$ & 0.50 & 0.08 \\
\hline \multirow{2}{*}{$\begin{array}{c}\text { Grip Strength } \\
\text { (Non - Dominant Upper Limb) }\end{array}$} & $\begin{array}{l}\text { DEMMI } \\
(\mathrm{n}=20)\end{array}$ & 0.28 & 0.23 \\
\hline & $\begin{array}{l}\text { 6-MWT } \\
(n=13)\end{array}$ & 0.35 & 0.24 \\
\hline $\begin{array}{c}\text { MIP } \\
\text { (Hospital Discharge) }\end{array}$ & $\begin{array}{l}\text { 6-MWT } \\
(\mathrm{n}=11)\end{array}$ & 0.75 & $0.01^{*}$ \\
\hline Respiratory Muscle Endurance & $\begin{array}{l}\text { 6-MWT } \\
(\mathrm{n}=11)\end{array}$ & 0.49 & 0.13 \\
\hline DEMMI & $\begin{array}{l}\text { 6-MWT } \\
(n=13)\end{array}$ & 0.23 & 0.45 \\
\hline \multirow{2}{*}{$\begin{array}{c}\text { HRQoL } \\
\text { (Vas Score) }\end{array}$} & $\begin{array}{l}\text { DEMMI } \\
(\mathrm{n}=17)\end{array}$ & 0.11 & 0.67 \\
\hline & $\begin{array}{l}\text { 6-MWT } \\
(n=13)\end{array}$ & -0.36 & 0.22 \\
\hline
\end{tabular}

MRC-SS - Medical Research Council - Sum Score; DEMMI - de Morton Mobility Index; 6-MWT - Six-Minute Walk Test; MIP - Maximal Inspiratory Pressure; VAS - Visual Analogue Scale; HRQoL - Health-Related Quality of Life

Spearman correlation coefficient was used to determine correlations

*Significant results, $\mathrm{P}<0.05$ 


\section{Chapter 5: Discussion of the Primary Research Study}

In this chapter, the findings of the primary research study will be discussed and interpreted, and the considerations for clinical practice will also be presented.

To my knowledge, this is the first study in a South African population to determine outcomes as well as the correlation between muscle structure, muscle function and physical function in critically ill patients at ICU discharge and at hospital discharge. Participants presented with changes in respiratory and peripheral muscle structure, muscle weakness of both the respiratory and peripheral muscles and reduced physical function. Despite presenting with impairments, majority of participants were satisfied with their health-related quality of life (HRQoL) at the time of hospital discharge. No correlations were found between muscle structure and muscle function or physical function at ICU awakening and at ICU discharge. Significant correlations were found between muscle function and physical function at hospital discharge.

\subsection{Characteristics of the cohort}

Our study population represented a young population, similar to a study that was conducted in a public hospital in Johannesburg South Africa, (81) but in contrast to other research studies conducted in developed countries. $(23,24,25,26,45,52)$ The possible reason for this finding is that our study and the study by Whelan, van Aswegen and Corner (81) were conducted in public hospitals where majority of the admissions are trauma-related as opposed to a developed country, where an older patient population with comorbidities would be admitted.

Our participants were classified as severely ill, but had a short duration of mechanical ventilation, ICU length of stay (LOS) and hospital LOS. Furthermore, only a minority of our 
participants received corticosteroids and were exposed to sedation for a short duration. These findings are also comparable to the study conducted by Whelan, van Aswegen and Corner (81), but in contrast to other research studies. $(23,24,25,26,45,52)$ This contrast is probably as a result of different admission diagnosis and/or a high demand for hospital beds. In our study, majority of admissions to the intensive care unit (ICU) were trauma-related resulting in a high demand for ICU beds whereas in other research studies, admission to the ICUs were mainly medical diagnosis such as Acute Respiratory Distress Syndrome (ARDS), sepsis and pneumonia.

\subsection{Outcomes and associations between muscle structure, muscle function and physical function}

\subsubsection{Muscle structure}

This study revealed that all muscles may not be equally affected by critical illness. A large variation in the changes of diaphragm thickness (Tdi) was not seen, as majority of our participants presented with a less than $10 \%(<10 \%)$ decrease in Tdi as compared to the studies by Goligher et al. $(3,60)$ The small decrease in Tdi could be due to the smaller sample size in our study, limited exposure of sedation to participants, the use of partially assisted modes of ventilation in our ICUs as well as a short duration of full ventilation in our study population.

Changes in peripheral muscle structure was more marked in the RF muscle thickness and cross-sectional area (CSA) as compared to the $\mathrm{VI}$ muscle, but an increase in echogenicity was seen in the VI muscle. RF is a fast twitch muscle (Type II) designed to assist in fast movements whereas, the VI muscle is a stabiliser muscle designed to maintain dynamic balance. Immobilisation studies have reported greater loss of fast twitch (type II) muscle fibres and a conversion from slow twitch (type I) muscle fibres to fast twitch (type II) muscle fibres in postural muscles. $(26,65)$ Therefore, the different functional roles of muscles may 
be the possible reason for the muscle loss in RF muscle thickness and CSA and the increase in echogenicity of the VI muscle observed in our study. Similar results were also obtained in the studies conducted by Hayes et al. (25) and Parry et al. (26). However, in these two studies ultrasonography was performed between day three and day seven, and the average length of ventilation (LOV) and ICU LOS was 10 and 20 days respectively. $(25,26)$ In our study, ultrasonography was performed from day one to day three and our participants had a shorter duration of mechanical ventilation and ICU LOS, yet presented with changes in RF and VI quantity and quality.

Parry et al. (26) found a significant inverse correlation between VI muscle echogenicity and physical function measures such as the Physical Function Intensive Care Test score (PFITs) and the ICU Mobility Scale (IMS) at ICU awakening. In contrast, in our study, a very weak inverse non-significant correlation was obtained between RF muscle echogenicity with the MRC-SS and the CPAx score and a weak inverse non-significant correlation was obtained between VI muscle echogenicity with the MRC-SS and the CPAx score. Therefore, it is unknown if the quality of the muscle worsens, strength of the peripheral muscles weakens and thus physical function reduces in critically ill survivors. However, other factors such as severity of illness and the exposure to corticosteroids and sedation may play a role. In our study, participants were classified as severely ill, were administered corticosteroids and were sedated with mainly propofol and fentanyl. It has been proposed that the use of corticosteroids and sedative agents like propofol may cause muscle atrophy and a decrease in muscle excitability, worsening the effect of bed rest and resulting in reduced physical function. $(5,17,95)$

\subsubsection{Muscle strength and function}

In a study conducted by Baldwin and Bersten (23), they reported that all their critically ill survivors presented with weakness in their elbow flexion, knee extension and handgrip force as determined by dynamometry and that fifty percent of their critically ill survivors also presented with intensive care unit-acquired weakness (ICU-AW). In our study, less than fifty 
percent of the participants presented with ICU-AW, but all our participants presented with weakness in their knee extension and handgrip force when compared to predictive and normative values. $(96,97)$ The difference between our study and the study conducted by Baldwin and Bersten (23) was that they measured muscle strength in a sample of critically ill survivors of sepsis whereas in our study, muscle strength was measured in a mixed cohort of trauma, surgical and medical participants. Furthermore, their critically ill survivors were exposed to corticosteroids and neuromuscular blocking agents (NMBA) for a longer duration as compared to our study. It has been postulated that corticosteroids and NMBA may cause skeletal muscle atrophy and myopathy, but research has reported conflicting conclusions regarding the associations between corticosteroids and NMBA with muscle function. $(95,98,99)$ However, our study did not record if participants received NMBA nor looked at the relationship between these factors.

A decrease in respiratory muscle strength and reduced respiratory muscle endurance was observed in our participants when compared to the ATS/ERS guidelines and norms of a healthy population. $(6,100)$ A study conducted by Bisset et al. (50) found that critically ill patients who were mechanically ventilated for at least seven days presented with reduced respiratory muscle endurance. In contrast, our participants were mechanically ventilated for a short duration of only three days. However, it has been previously reported that mechanical ventilation $\geq 48$ hours results in atrophy of the diaphragm muscle fibres contributing to a reduction in respiratory muscle strength and endurance. (101) Furthermore, our study was the first study to have measured respiratory muscle endurance with participants having to continuously breathe against an inspiratory load set at $50 \%$ of their MIP whereas, in the study by Bisset et al. (50), they determined respiratory muscle endurance using the Fatigue Resistance Index (FRI). Although there was a difference in testing between our study and the study by Bisset et al. (50), both studies found reduced respiratory muscle endurance in critically ill survivors. 


\subsubsection{Physical function}

Our participants presented with reduced physical function at ICU awakening and at ICU discharge regardless of a short ICU LOS. In a study conducted by Whelan, van Aswegen and Corner (81), the CPAx scores improved at ICU discharge when compared to the CPAX scores at ICU admission. In our study, similar results were obtained where the CPAx scores also improved at ICU discharge when compared to ICU awakening, but the improvement in CPAx scores was better in the study by Whelan, van Aswegen and Corner (81). The difference in CPAx scores between our study and the study by Whelan, van Aswegen and Corner $(81)$ is due to the fact that their participants received rehabilitation daily during their ICU stay whereas in our study, data regarding whether participants received rehabilitation was not captured. In addition, our participants presented with changes in muscle structure and weakness of the respiratory and peripheral muscles. However, there were no correlations found between muscle structure and function with physical function at ICU awakening and at ICU discharge in our study. Therefore, the reduced physical function seen in our participants could be as a result of other factors such as severity of illness, age, the LOV and ICU LOS. However, these factors will need to be investigated in future studies.

Participants scored maximally on the DEMMI demonstrating improved physical function at hospital discharge when compared to ICU awakening and ICU discharge. The possible reason for participants performing better could be due to the DEMMI assessing less domains of physical function, but examines these domains of physical function differently when compared to the CPAx. A significant correlation was found between the MRC-SS and the DEMMI and implies that as the strength of the peripheral muscles increases, physical function will improve in critically ill survivors. A similar correlation was found between the isometric knee extension force of the non-dominant limb and the DEMMI. This is the first study to find such a correlation and could be due to certain participants scoring better on the isometric knee extension force of the non-dominant limb and the small difference in isometric knee extension force scores between the dominant and non-dominant lower limb. 
This may also indicate that improvement in muscle strength will result in an improvement in physical function regardless of it being a dominant or non-dominant limb.

Participants walked a longer six-minute walk distance (6-MWD) when performing the second six-minute walk test (6-MWT). This could be due to the "learning effect" of the test by the participants. Regardless of this improvement in the second 6-MWD, participants still presented with reduced functional exercise capacity when compared to norms of a healthy population. (102) The reduced functional exercise capacity seen in our participants indicates that our participants may experience difficulty with walking and carrying out activities of daily living (ADLs) once they return to normal life roles. A similar finding was found in a study conducted by Alison et al. (86) where participants walked a longer 6-MWD in the second 6MWT also due to the learning effect of the test. In comparison, our participants performed the 6-MWT at hospital discharge whereas in other research studies, critically ill survivors performed the 6-MWT at three, six- and twelve-months post-hospital discharge. $(86,103)$ When comparing our results to other research studies whereby participants performed the 6MWT post-hospital discharge, our participants perfomed much worse in the 6-MWT at hospital discharge.

The relationship between respiratory muscle strength and the 6-MWT has been extensively studied in the chronic obstructive pulmonary disease (COPD) population and healthy elderly population. $(104,105,106)$ To my knowledge, ours is the first study to describe a positive significant correlation between maximal inspiratory pressure (MIP) and the 6-MWT and a moderate positive correlation between the MRC-SS and the 6-MWT in critically ill survivors. This indicates that improvement in inspiratory muscle strength may lead to better oxygen consumption, reducing respiratory and peripheral muscle fatigue, resulting in improved physical function and exercise tolerance. Similarly, as the strength of the peripheral muscle improves, this may lead to a better functional exercise capacity in critically ill survivors. 
This study was also the first study to find a moderate positive correlation between respiratory muscle endurance and the 6-MWT. The diaphragm mainly comprises of slow twitch muscle fibres as it engages in continuous rhythmic activity which does not permit any pause and therefore, must be very resistant to fatigue. (107) The 6-MWT entails continuously walking a 30-metre distance for six-minutes and therefore, as the endurance of the respiratory muscles improves, a longer 6-MWD may be achieved. Thus, exercise training focusing on respiratory muscle strength and endurance may improve physical function in critically ill survivors.

\subsubsection{Health-related quality of life (HRQoL)}

Participants perceived their overall health as being satisfactory although there were a few participants that indicated having problems in the five dimensions of health, despite the entire cohort presenting with impairments in muscle structure and function and reduced physical function. A possible explanation for participants being satisfied with their HRQoL is that HRQoL is subjective and that participants are grateful for being "alive". In studies conducted by Cuthbertson et al. $(108,109)$ it was found that critically ill survivors reported having a poor HRQoL only one year after their critical illness, due to presenting with physical function disabilities, cognitive and/or mental dysfunction. Interestingly, in our study a weak correlation and an inverse correlation was found between participant perception of health with physical function suggesting that it is not known whether improvements in HRQoL will lead to improved or worsening physical function in critically ill survivors. Therefore, it can be postulated that our participants perception of their health may change once they return to normal life roles, resulting in a reduced physical function and a poorer HRQoL.

\subsection{Consideration for clinical practice}

Critically ill survivors present with changes in respiratory and peripheral muscle structure, weakness of the respiratory and peripheral muscles and reduced physical function early during the course of critical illness. Although, our participants were classified as severely ill 
and majority of the admissions to the ICUs were trauma-related, our participants were mechanically ventilated for a shorter duration, resulting in a short ICU and hospital LOS. The use of partially assisted modes of ventilation and a short duration of full ventilation in our ICUs may have attributed to minor changes in the Tdi in our participants. In addition, only a minority of our participants were exposed to sedation and/or administered corticosteroids. Despite limited exposures to sedation and corticosteroids, and a short LOV, ICU and hospital LOS, there were still notable impairments in muscle structure, muscle function and physical function.

An improvement in physical function may be as a result of improvement in muscle function. Therefore, rehabilitation focusing on muscle function and physical function should commence early in the ICU and throughout a continuum. However, specific protocols regarding optimal timing, dosage and progression of exercises still needs to be determined. It is also important to note that critical illness affects various types of skeletal muscles differently and thus future rehabilitation-based interventions may need to factor in the type of muscle affected and the timing for these rehabilitation-based interventions. Therefore, regular assessments of muscle structure and function and physical function is needed and may assist in the development of future rehabilitation-based interventions.

Grip strength as measured using hand-held dynamometry could be used to determine peripheral muscle strength instead of the MRC-SS in critically ill patients. Although both of these volitional tests require a patient to be awake, alert and orientated, the use of handheld dynamometry will not involve the extensive training that the MRC-SS requires and will take less time for clinicians to conduct the test and for patients to complete the test. 


\section{Chapter 6: Overall Conclusion}

In this chapter, the limitations of the primary research study, recommendation for future research as well as an overall thesis conclusion will be presented.

\subsection{Limitations}

\subsubsection{Primary study}

The findings of the primary research study are subject to a few limitations. Ultrasonography was only performed during the participants ICU stay and not at ICU discharge or hospital discharge. Therefore, it is unknown whether changes in Tdi, quadriceps muscle thickness and quadriceps muscle echogenicity remained the same, improved or worsened at ICU discharge or at hospital discharge. Furthermore, no correlations between muscle structure and muscle function or muscle structure and physical function could be determined at ICU discharge and hospital discharge. Participants were included but had to be excluded from the study due to unplanned/or early extubation or being transferred back to the referring hospitals. This resulted in a lost to follow-up of participants, lost data and smaller sample size to determine correlations between muscle structure, muscle function and physical function.

Majority of our study population were male participants and thus it is unknown whether critical illness affects gender equally or differently with regards to muscle structure, muscle function, physical function and HRQoL. Our study did not plan to record whether participants received neuromuscular-blocking agents (NMBA) during their ICU care and therefore, do not know the impact NMBA has on muscle structure and muscle function. Participants were not allowed any practice breaths when measuring MIP and respiratory muscle endurance in order to prevent fatigue or familiarise themselves with the equipment. This could have resulted in submaximal breaths due to the lack of understanding. 


\subsection{Recommendation for future research}

The aim of this study was to determine the correlation between muscle structure, muscle function and physical function in critically ill patients at ICU discharge and at hospital discharge. The strength of the correlations found between muscle structure and muscle function suggests that structural changes seen in muscles early during critical illness does not necessarily mean that muscles will not be able to function. Therefore, it would be interesting to compare structural changes in muscles in a group of critically ill patients with ICU-AW and to a group of critically ill patients that scored more than 48 on the MRC-SS at ICU discharge. Furthermore, predictors of muscle structure, muscle function and physical function in critically ill patients should be investigated. The standardisation of the methodology for the assessment tools for muscle structure and function and physical function should be explored. This will also assist in determining if impairments in muscle structure translates to impairments in muscle function or even physical function.

Future research should also investigate the trajectory of critically ill patients regarding muscle structure and function and physical function from ICU admission to post-hospital discharge. Rehabilitation-based interventions could potentially impact muscle strength. Therefore, the effects of rehabilitation-based interventions on muscle structure, muscle function and physical function in critically ill patients should be investigated. Finally, the effects of different lung mechanics and ventilator settings on Tdi and diaphragm contractile activity should also be investigated.

\subsection{Overall conclusion}

Our current understanding of muscle structure and muscle function in critically ill patients is still evolving (Chapter 2). After scoping the literature, discrepancies were found in the methodologies used to investigate muscle structure and muscle function, inconsistency in the timing, i.e., when measurements were to be taken, as well as the uncertainty of what should be measured which overall hampered our current understanding. Furthermore, the 
focus of research in recent years has been centred on exploring muscle structure and very little on muscle function.

In our South African cohort of critically ill survivors, it was found that changes in respiratory and peripheral muscle structure, weakness of the respiratory and peripheral muscles, reduced respiratory muscle endurance and reduced physical function were a common manifestation that occurred during critical illness (Chapter 4). However, the correlations found between muscle structure, muscle function and physical function at ICU awakening and at ICU discharge were weak. Our results indicate that while the correlations found between muscle function and physical function were stronger at hospital discharge, changes in muscle structure and muscle function can only partially explain the poor performance in physical function in critically ill survivors. Furthermore, decrease muscle function and reduced physical function does not correlate with patients' perception of their HRQoL at hospital discharge. Therefore, this means that clinicians will need to provide critically ill survivors with targeted and realistic information about what to expect when returning to their normal life roles. Moving forward it will be important for researchers to identify other variables affecting physical function in critically ill survivors. If these variables are amenable to physical function, then a better understanding of physical function outcomes in critically ill survivors may be achieved. 


\section{References}

1. Knaus WA, Draper EA, Wagner DP, Zimmerman JE. APACHE II: A severity of disease classification system. Crit Care Med. 1985;13(10):818-29.

2. Matamis D, Soilemezi E, Tsagourias M, Akoumianaki E, Dimassi S, Boroli F, et al. Sonographic evaluation of the diaphragm in critically ill patients. Technique and clinical applications. Intensive Care Med. 2013;39(5):801-10.

3. Goligher EC, Fan E, Herridge MS, Murray A, Vorona S, Brace D, et al. Evolution of diaphragm thickness during mechanical ventilation: Impact of inspiratory effort. Am J Respir Crit Care Med. 2015;192(9):1080-8.

4. Baldwin CE, Paratz JD, Bersten AD. Muscle strength assessment in critically ill patients with handheld dynamometry: An investigation of reliability, minimal detectable change, and time to peak force generation. J Crit Care [Internet]. 2013;28(1):77-86. Available from: http://dx.doi.org/10.1016/j.jcrc.2012.03.001.

5. Piva S, Fagoni N, Latronico N. Intensive care unit-acquired weakness: unanswered questions and targets for future research. F1000Research. 2019;8(508):1-10.

6. Gibson GJ, Whitelaw W, Siafakas N. ATS/ERS Statement on respiratory muscle testing. Am J Respir Crit Care Med. 2002;166(4):518-624.

7. Schoser B, Fong E, Geberhiwot T, Hughes D, Kissel JT, Madathil SC, et al. Maximum inspiratory pressure as a clinically meaningful trial endpoint for neuromuscular diseases: A comprehensive review of the literature. Orphanet $\mathrm{J}$ Rare Dis. 2017;12(1):1-12.

8. Hermans G, Van Aerde N, Meersseman P, Van Mechelen H, Debaveye Y, Wilmer A, et al. Five-year mortality and morbidity impact of prolonged versus brief ICU stay: a propensity score matched cohort study. Thorax. 2019;74(11):1037-45.

9. Dettling-Ihnenfeldt DS, Wieske L, Horn J, Nollet F, Van Der Schaaf M. Functional recovery in patients with and without intensive care unit-acquired weakness. Am J Phys Med Rehabil. 2017;96(4):236-42. 
10. Inoue S, Hatakeyama J, Kondo Y, Hifumi T, Sakuramoto H, Kawasaki T, et al. Postintensive care syndrome: its pathophysiology, prevention, and future directions. Acute Med Surg. 2019;6(10):233-46.

11. Wieske L, Dettling-Ihnenfeldt DS, Verhamme C, Nollet F, van Schaik IN, Schultz MJ, et al. Impact of ICU-acquired weakness on post-ICU physical functioning: A follow-up study. Crit Care. 2015;19(1):1-8.

12. Bemis-dougherty AR, Smith JM. What follows survival of critical illness? Physical therapists' management of patients with post- intensive care syndrome. 2013;93(2):179-85.

13. Daniels LM, Johnson AB, Cornelius PJ, Bowron C, Lehnertz A, Moore M, et al. Improving quality of life in patients at risk for post-intensive care syndrome. Mayo Clin Proc Innov Qual Outcomes [Internet]. 2018;2(4):359-69. Available from: https://doi.org/10.1016/j.mayocpiqo.2018.10.001.

14. Kondo Y, Fuke R, Hifumi T, Hatakeyama J, Takei T, Yamakawa K, et al. Early rehabilitation for the prevention of postintensive care syndrome in critically ill patients: a study protocol for a systematic review and meta-analysis. BMJ Open. 2017;7(3):1-6.

15. Farhan H, Latronico N, Zafonte R, Eikermann M, Moreno-Duarte I. Acquired muscle weakness in the surgical intensive care unit. Anesthesiology. 2015;124(1):207-34.

16. Puthucheary Z. An update on muscle wasting in ICU. Signa Vitae. 2017;13(3):30-1.

17. Schreiber A, Bertoni M, Goligher EC. Avoiding respiratory and peripheral muscle injury during mechanical ventilation: Diaphragm-protective ventilation and early mobilisation. Crit Care Clin. 2018;34(3):357-81.

18. Barreiro E. Models of disuse muscle atrophy: therapeutic implications in critically ill patients. Ann Transl Med. 2018;6(2):29-29.

19. Batt J, Herridge MS, Dos Santos CC. From skeletal muscle weakness to functional outcomes following critical illness: a translational biology perspective. Thorax. 2019;74(11):1091-8. 
20. Fan E, Dowdy DW, Colantuoni E, Pedro A, Sevransky JE, Shanholtz C, et al. Physical complications in acute lung injury survivors: A 2-year longitudinal prospective study. 2015;42(4):849-59.

21. Herridge MS, Tansay CM, Matte A, Tomlinson G, Diaz-Granados N, Cooper A, et al. Functional disability 5 years after acute respiratory distress syndrome. Heart Fail. 2005;36(14):225-37.

22. Puthucheary Z, Montgomery H, Moxham J, Harridge S, Hart N. Structure to function: Muscle failure in critically ill patients. J Physiol. 2010;588(23):4641-8.

23. Baldwin CE, Bersten AD. Alterations in respiratory and limb muscle strength and size in patients with sepsis who are mechanically ventilated. Phys Ther. 2014;94(1):68-82.

24. De Jonghe B, Bastuji-Garin S, Durand M, Malissin I, Rodrigues P, Charles C, et al. Respiratory weakness is associated with limb weakness and delayed weaning in critical illness. Crit Care Med. 2007;35(9):2007-15.

25. Hayes K, Holland AE, Pellegrino VA, Mathur S, Hodgson CL. Acute skeletal muscle wasting and relation to physical function in patients requiring extracorporeal membrane oxygenation (ECMO). J Crit Care [Internet]. 2018;48(8):1-8. Available from: https://doi.org/10.1016/j.jcrc.2018.08.002.

26. Parry SM, El-Ansary D, Cartwright MS, Sarwal A, Berney S, Koopman R, et al. Ultrasonography in the intensive care setting can be used to detect changes in the quality and quantity of muscle and is related to muscle strength and function. J Crit Care. 2015;30(5):1151.e9-1151.e14.

27. Cuthbertson BH, Goddard S. Benefits and harms of early rehabilitation. Intensive Care Med. 2017;43(12):1878-80.

28. Files DC, Sanchez MA, Morris PE. A conceptual framework: The early and late phases of skeletal muscle dysfunction in the acute respiratory distress syndrome. Crit Care [Internet]. 2015;19(1):1-10. Available from: http://dx.doi.org/10.1186/s13054-015-0979-5. 
29. Bouglé A, Rocheteau P, Sharshar T, Chrétien F. Muscle regeneration after sepsis. Crit Care [Internet]. 2016;20(1):16-7. Available from: http://dx.doi.org/10.1186/s13054-016-1308-3.

30. Scott W, Stevens J, Binder-Macleod SA. Human skeletal muscle fiber type classifications. Phys Ther. 2001;81(11):1810-6.

31. Arksey H, O'Malley L. Scoping studies: Towards a methodological framework. Int J Soc Res Methodol. 2008;8(1):19-32.

32. Buscher $H$, Valta $P$, Boie $T$, Hinz J, Moerer $O$, Sydow M, et al. Assessment of diaphragmatic function with cervical magnetic stimulation of critically ill patients. Anaesth Intensive Care. 2005;33(4):483-91.

33. Ginz HF, laizzo PA, Girard T, Urwyler A, Pargger H. Decreased isometric skeletal muscle force in critically ill patients. Swiss Med Wkly. 2005;135(37-38):555-61.

34. Klaude M, Hammarqvist F, Wemerman J. An assay of microsomal membraneassociated proteasomes demonstrates increased proteolytic activity in skeletal muscle of intensive care unit patients. Clin Nutr. 2005;24(2):259-65.

35. Fredriksson $\mathrm{K}$, Hammarqvist $\mathrm{F}$, Strigård $\mathrm{K}$, Hultenby $\mathrm{K}$, Ljungqvist $\mathrm{O}$, Wernerman $\mathrm{J}$, et al. Derangements in mitochondrial metabolism in intercostal and leg muscle of critically ill patients with sepsis-induced multiple organ failure. Am J Physiol Endocrinol Metab. 2006;291(5):1044-50.

36. Ginz HF, laizzo PA, Urwyler A, Pargger H. Use of non-invasive-stimulated muscle force assessment in long-term critically ill patients: A future standard in the intensive care unit? Acta Anaesthesiol Scand. 2008;52(1):20-7.

37. Weber-Carstens S, Koch S, Spuler S, Spies CD, Bubser F, Wernecke KD, et al. Nonexcitable muscle membrane predicts intensive care unit-acquired paresis in mechanically ventilated, sedated patients. Crit Care Med. 2009;37(9):2632-7.

38. Hermans G, Agten A, Testelmans D, Decramer M, Gayan-Ramirez G. Increased duration of mechanical ventilation is associated with decreased diaphragmatic force: A prospective observational study. Crit Care [Internet]. 2010;14(4):1-10. 
Available from:

http://www.embase.com/search/results?subaction=viewrecord\&from=export\&id=L5

0980161\%5Cnhttp://ccforum.com/content/14/4/R127\%5Cnhttp://dx.doi.org/10.1186/

cc9094\%5Cnhttp://linksource.ebsco.com/linking.aspx?sid=EMBASE\&issn=1364853

5\&id=doi:10.1186/cc9094\&atitl.

39. Jaber S, Petrof BJ, Jung B, Chanques G, Berthet JP, Rabuel C, et al. Rapidly progressive diaphragmatic weakness and injury during mechanical ventilation in humans. Am J Respir Crit Care Med. 2011;183(3):364-71.

40. Bierbrauer J, Koch S, Olbricht C, Hamati J, Lodka D, Schneider J, et al. Early type II fiber atrophy in intensive care unit patients with nonexcitable muscle membrane. Crit Care Med. 2012;40(2):647-50.

41. Derde S, Hermans G, Derese I, Güiza F, Hedström Y, Wouters PJ, et al. Muscle atrophy and preferential loss of myosin in prolonged critically ill Patients. Crit Care Med. 2012;40(1):79-89.

42. Llano-Diez M, Renaud G, Andersson M, Marrero HG, Cacciani N, Engquist $\mathrm{H}$, et al. Mechanisms underlying ICU muscle wasting and effects of passive mechanical loading. Crit Care. 2012;16(5):1-16.

43. Cartwright MS, Kwayisi G, Griffin LP, Sarwal A, Walker FO, Harris JM, et al. Quantitative neuromuscular ultrasound in the intensive care unit. Muscle and Nerve. $2013 ; 47(2): 255-9$.

44. Demoule A, Jung B, Prodanovic H, Molinari N, Chanques G, Coirault C, et al. Diaphragm dysfunction on admission to the intensive care unit: Prevalence, risk factors, and prognostic impact - A prospective study. Am J Respir Crit Care Med. 2013;188(2):213-9.

45. Puthucheary ZA, Rawal J, McPhail M, Connolly B, Ratnayake G, Chan P, et al. Acute skeletal muscle wasting in critical illness. JAMA - J Am Med Assoc. 2013;310(15):1591-600. 
46. Supinski GS, Ann Callahan L. Diaphragm weakness in mechanically ventilated critically ill patients. Crit Care. 2013;17(3):1-17.

47. Weber-Carstens S, Schneider J, Wollersheim T, Assmann A, Bierbrauer J, Marg A, et al. Critical illness myopathy and GLUT4 significance of insulin and muscle contraction. Am J Respir Crit Care Med. 2013;187(4):387-96.

48. Sheean PM, Peterson SJ, Perez SG, Troy KL, Patel A, Sclamberg JS, et al. The prevalance of sarcopenia in patients with respiratory failure classified as normal nourished using subjective global assessment and computed tomograpghy. Bone. 2014;23(1):1-7.

49. Wollersheim T, Woehlecke J, Krebs M, Hamati J, Lodka D, Luther-Schroeder A, et al. Dynamics of myosin degradation in intensive care unit-acquired weakness during severe critical illness. Intensive Care Med. 2014;40(4):528-39.

50. Bissett B, Leditschke IA, Neeman T, Boots R, Paratz J. Weaned but weary: One third of adult intensive care patients mechanically ventilated for 7 days or more have impaired inspiratory muscle endurance after successful weaning. Hear Lung $\mathrm{J}$ Acute Crit Care [Internet]. 2015;44(1):15-20. Available from:

http://dx.doi.org/10.1016/j.hrtlng.2014.10.001.

51. Hooijman PE, Beishuizen A, Witt CC, de Waard MC, Girbes ARJ, Spoelstra-de Man AME, et al. Diaphragm muscle fiber weakness and ubiquitin-proteasome activation in critically ill patients. Am J Respir Crit Care Med. 2015;191(10):1126-38.

52. Puthucheary ZA, Phadke R, Rawal J, McPhail MJW, Sidhu PS, Rowlerson A, et al. Qualitative ultrasound in acute critical illness muscle wasting. Crit Care Med. 2015;43(8):1603-11.

53. Schepens T, Verbrugghe W, Dams K, Corthouts B, Parizel PM, Jorens PG. The course of diaphragm atrophy in ventilated patients assessed with ultrasound: A longitudinal cohort study. Crit Care [Internet]. 2015;19(1):1-8. Available from: http://dx.doi.org/10.1186/s13054-015-1141-0. 
54. Umbrello M, Formenti P, Longhi D, Galimberti A, Piva I, Pezzi A, et al. Diaphragm ultrasound as indicator of respiratory effort in critically ill patients undergoing assisted mechanical ventilation: A pilot clinical study. Crit Care. 2015;19(1):1-10.

55. Demoule A, Molinari N, Jung B, Prodanovic H, Chanques G, Matecki S, et al. Patterns of diaphragm function in critically ill patients receiving prolonged mechanical ventilation: A prospective longitudinal study. Ann Intensive Care. $2016 ; 6(1): 1-8$.

56. Lee GD, Kim HC, Yoo JW, Lee SJ, Cho YJ, Bae K, et al. Computed tomography confirms a reduction in diaphragm thickness in mechanically ventilated patients. $\mathrm{J}$ Crit Care [Internet]. 2016;33(10):47-50. Available from: http://dx.doi.org/10.1016/j.jcrc.2016.02.013.

57. Turton P, Hay R, Taylor J, McPhee J, Welters I. Human limb skeletal muscle wasting and architectural remodeling during five to ten days intubation and ventilation in critical care - an observational study using ultrasound. BMC Anesthesiol [Internet]. 2016;16(1):1-8. Available from: http://dx.doi.org/10.1186/s12871-016-0269-z.

58. Zambon M, Beccaria P, Matsuno J, Gemma M, Frati E, Colombo S, et al. Mechanical ventilation and diaphragmatic atrophy in critically ill patients: An ultrasound study. Crit Care Med. 2016;44(7):1347-52.

59. Van den Berg M, Hooijman PE, Beishuizen A, De Waard MC, Paul MA, Hartemink KJ, et al. Diaphragm atrophy and weakness in the absence of mitochondrial dysfunction in the critically III. Am J Respir Crit Care Med. 2017;196(12):1544-58.

60. Goligher EC, Dres M, Fan E, Rubenfeld GD, Scales DC, Herridge MS, et al. Mechanical ventilation-induced diaphragm atrophy strongly impacts clinical outcomes. Am J Respir Crit Care Med. 2018;197(2):204-13.

61. Hernández-Socorro CR, Saavedra P, López-Fernández JC, Ruiz-Santana S. Assessment of muscle wasting in long-stay ICU patients using a new ultrasound protocol. Nutrients. 2018;10(12):1-11. 
62. Puthucheary ZA, Astin R, McPhail MJW, Saeed S, Pasha Y, Bear DE, et al. Metabolic phenotype of skeletal muscle in early critical illness. Thorax. 2018;73(10):926-35.

63. Desai S V., Law TJ, Needham DM. Long-term complications of critical care. Crit Care Med. 2011;39(2):371-9.

64. Hermans G, Clerckx B, Vanhullebusch T, Segers J, Vanpee G, Robbeets C, et al. Interobserver agreement of medical research council sum score and handgrip strength in the intensive care unit. Muscle and Nerve. 2012;45(1):18-25.

65. Parry SM, Puthucheary ZA. The impact of extended bed rest on the musculoskeletal system in the critical care environment. Extrem Physiol Med. 2015;4(1):1-8.

66. Zambon M, Greco M, Bocchino S, Cabrini L, Beccaria PF, Zangrillo A. Assessment of diaphragmatic dysfunction in the critically ill patient with ultrasound: a systematic review. Intensive Care Med. 2017;43(1):29-38.

67. Dinino E, Gartman EJ, Sethi JM, McCool FD. Diaphragm ultrasound as a predictor of successful extubation from mechanical ventilation. Thorax. 2014;69(5):423-7.

68. Farghaly S, Hasan AA. Diaphragm ultrasound as a new method to predict extubation outcome in mechanically ventilated patients. Aust Crit Care [Internet]. 2017;30(1):37-43. Available from: http://dx.doi.org/10.1016/j.aucc.2016.03.004.

69. Ferrari G, De Filippi G, Elia F, Panero F, Volpicelli G, Aprà F. Diaphragm ultrasound as a new index of discontinuation from mechanical ventilation. Crit Ultrasound J. $2014 ; 6(1): 1-6$.

70. Francis CA, Hoffer JA, Reynolds S. Ultrasonography evaluation of diaphragm thickness during mechanical ventilation in intensive care patients. Am journnal Crit care. $2016 ; 25(1): 1-9$.

71. Sarwal A, Parry SM, Berry MJ, Hsu FC, Lewis MT, Justus NW, et al. Interobserver reliability of quantitative muscle sonographic analysis in the critically ill population. $\mathrm{J}$ Ultrasound Med. 2015;34(7):1191-200. 
72. Baldwin CE, Paratz JD, Bersten AD. Diaphragm and peripheral muscle thickness on ultrasound: Intra-rater reliability and variability of a methodology using nonstandard recumbent positions. Respirology. 2011;16(7):1136-43.

73. Connolly BA, Jones GD, Curtis AA, Murphy PB, Douiri A, Hopkinson NS, et al. Clinical predictive value of manual muscle strength testing during critical illness : an observational cohort study. 2013;17(5):1-9.

74. Hough CL, Lieu BK, Caldwell ES. Manual muscle strength testing of critically ill patients : feasibility and interobserver agreement. Crit Care. 2011;15(1):1-7.

75. Charususin N, Gosselink R, Decramer M, McConnell A, Saey D, Maltais F, et al. Inspiratory muscle training protocol for patients with chronic obstructive pulmonary disease (IMTCO study): A multicentre randomised controlled trial. BMJ Open. 2013;3(8):1-7.

76. Medrinal C, Prieur G, Combret Y, Quesada AR, Bonnevie T, Gravier FE, et al. Reliability of respiratory pressure measurements in ventilated and non-ventilated patients in ICU: an observational study. Ann Intensive Care [Internet]. 2018;8(1):48. Available from: https://doi.org/10.1186/s13613-018-0362-1.

77. Formiga M, Roach K, Vital I, Urdaneta G, Balestrini K, Calderon-Candelario R, et al. Reliability and validity of the test of incremental respiratory endurance measures of inspiratory muscle performance in COPD. Int J COPD [Internet]. 2018;13(10):1569_ 76. Available from: http://www.embase.com/search/results?subaction=viewrecord\&from=export\&id=L6 22142294\%0Ahttp://dx.doi.org/10.2147/COPD.S160512.

78. Samosawala NR, Vaishali K, Kalyana BC. Measurement of muscle strength with handheld dynamometer in Intensive Care Unit. Indian J Crit Care Med. 2016;20(1):21-6.

79. Vanpee G, Segers J, Van Mechelen H, Wouters P, Van Den Berghe G, Hermans G, et al. The interobserver agreement of handheld dynamometry for muscle strength assessment in critically ill patients. Crit Care Med. 2011;39(8):1929-34. 
80. Corner EJ, Wood H, Englebretsen C, Thomas A, Grant RL, Nikoletou D, et al. The Chelsea Critical Care Physical Assessment tool (CPAx): Validation of an innovative new tool to measure physical morbidity in the general adult critical care population; An observational proof-of-concept pilot study. Physiother (United Kingdom) [Internet]. 2013;99(1):33-41. Available from: http://dx.doi.org/10.1016/j.physio.2012.01.003.

81. Whelan M, van Aswegen H, Corner E. Impact of the Chelsea Critical Care Physical Assessment (CPAx) tool on clinical outcomes of surgical and trauma patients in an intensive care unit: An experimental study. South African J Physiother. 2018;74(1):1-8.

82. Corner EJ, Soni N, Handy JM, Brett SJ. Construct validity of the Chelsea Critical Care Physical Assessment tool: An observational study of recovery from critical illness. Crit Care. 2014;18(2):1-10.

83. Corner EJ, Hichens L V., Attrill KM, Vizcaychipi MP, Brett SJ, Handy JM. The responsiveness of the Chelsea Critical Care Physical Assessment tool in measuring functional recovery in the burns critical care population: An observational study. Burns [Internet]. 2015;41(2):241-7. Available from: http://dx.doi.org/10.1016/j.burns.2014.12.002.

84. New PW, Scroggie GD, Williams CM. The validity, reliability, responsiveness and minimal clinically important difference of the de Morton mobility index in rehabilitation. Disabil Rehabil. 2017;39(10):1039-43.

85. Sommer J, Tom V, Robert L, Frans N, Engelbert RHH, Van der Schaaf M. de Morton Mobility Index is feasible, relaible and valid in patients with critical illness. Am Phys Ther Assoc. 2016;96(10):1658-66.

86. Alison JA, Kenny P, King MT, Mckinley S, Aitken LM, Leslie GD, et al. Repeatability of the six-minute walk test and relation to physical function in survivors of a critical illness. Phys Ther. 2012;92(12):1556-63.

87. Burtin C, Clerckx B, Robbeets C, Ferdinande P, Langer D, Troosters T, et al. Early 
exercise in critically ill patients enhances short-term functional recovery. Crit Care Med. 2009;37(9):2499-505.

88. Holland AE, Spruit MA, Troosters T, Puhan MA, Pepin V, Saey D, et al. An official European Respiratory Society/American Thoracic Society technical standard: field walking tests in chronic respiratory disease. Eur Respir J. 2014;44(6):1428-46.

89. Demers C, McKelvie RS, Negassa A, Yusuf S. Reliability, validity, and responsiveness of the six-minute walk test in patients with heart failure. Am Heart J. $2001 ; 142(4): 698-703$.

90. Chan KS, Pfoh ER, Denehy L, Elliott D, Holland AE, Dinglas VD, et al. Construct validity and minimal important diff erence of 6-minute walk distance in survivors of acute respiratory failure. Chest. 2015;147(5):1316-26.

91. Granja C, Teixeira-Pinto A, Costa-Pereira A. Quality of life after intensive care Evaluation with EQ-5D questionnaire. Intensive Care Med. 2002;28(7):898-907.

92. Li L, Liu C, Cai X, Yu H, Zeng X, Sui M, et al. Validity and reliability of the EQ-5D-5 L in family caregivers of leukemia patients. BMC Cancer. 2019;19(1):1-9.

93. Holtzhausen S, Unger M, Lupton-Smith A, Hanekom S. An investigation into the use of ultrasound as a surrogate measure of diaphragm function. Hear Lung [Internet]. 2018;47(4):418-24. Available from:

https://doi.org/10.1016/j.hrtlng.2018.04.010.

94. Crapo R, Enright P, Zeballos R. American Thoracic Society Statement: Guidelines for the six-minute walk test. Am J Respir Crit Care Med. 2002;166(1):111-7.

95. Schweickert WD, Hall J. ICU-acquired weakness. Chest. 2007;131(5):1541-9.

96. Bohannon RW. Reference values for extremity muscle strength obtained by handheld dynamometry from adults aged 20 to 79 years. Arch Phys Med Rehabil. 1997;78(1):26-32.

97. Pretorius B, Drennan D, Saunders E, Strachan K, Vermeulen E. The need for standardised South African power grip strength norms. South Africa Journal Occupational Ther. 2005;35(1):13-8. 
98. Jolley SE, Bunnell AE, Hough CL. ICU-acquired weakness. Chest [Internet]. 2016;150(5):1129-40. Available from: http://dx.doi.org/10.1016/j.chest.2016.06.006.

99. Walsh CJ, Batt J, Herridge MS, Dos Santos CC. Muscle wasting and early mobilization in acute respiratory distress syndrome. Clin Chest Med [Internet]. 2014;35(4):811-26. Available from: http://dx.doi.org/10.1016/j.ccm.2014.08.016.

100. Sclauser Pessoa IMB, Parreira VF, Fregonezi GAF, Sheel AW, Chung F, Reid WD. Reference values for maximal inspiratory pressure: A systematic review. Can Respir J. 2014;21(1):43-50.

101. Chang AT, Boots RJ, Brown MG, Paratz J, Hodges PW. Reduced Inspiratory Muscle Endurance Following Successful Weaning from Prolonged Mechanical Ventilation. Chest. 2005;128(2):553-9.

102. Ben Saad H, Prefaut C, Tabka Z, Hadj Mtir A, Chemit M, Hassaoune R, et al. 6Minute walk distance in healthy North Africans older than 40 years: Influence of parity. Respir Med. 2009;103(1):74-84.

103. Parry SM, Nalamalapu SR, Nunna K, Rabiee A, Friedman LA, Colantuoni E, et al. Six-minute walk distance after critical illness: A systematic review and metaanalysis. J Intensive Care Med. 2019;5(10):1-9.

104. Giua R, Pedone C, Scarlata S, Carrozzo I, Rossi FF, Valiani V, et al. Relationship between respiratory muscle strength and physical performance in elderly hospitalized patients. Rejuvenation Res. 2014;17(4):366-71.

105. Graur N, Weiner P, Kramer MR. Annals of physiotherapy clinics effects of inspiratory muscle training on respiratory muscle strength, functional capacity and health-related quality of life of patients following lung transplantation. Ann Physiother Clin. 2020;2(1):10-2.

106. Vilaça AF, Pedrosa BC de S, Amaral TCN, Andrade M do A, Castro CMMB de, França EET de. The effect of inspiratory muscle training on the quality of life, immune response, inspiratory and lower limb muscle strength of older adults: a randomized controlled trial. Rev Bras Geriatr e Gerontol. 2019;22(6):1-11. 
107. Polla B, D'Antona G, Bottinelli R, Reggiani C. Respiratory muscle fibres: Specialisation and plasticity. Thorax. 2004;59(9):808-17.

108. Cuthbertson BH, Roughton S, Jenkinson D, MacLennan G, Vale L. Quality of life in the five years after intensive care: A cohort study. Crit Care. 2010;14(1):1-12.

109. Cuthbertson BH, Elders A, Hall S, Taylor J, MacLennan G, Mackirdy F, et al. Mortality and quality of life in the five years after severe sepsis. Crit Care. 2013;17(2):1-8. 


\section{Addendums}

\section{Addendum A: Search Strategies of the Databases Searched}

\section{EBSCOhost - Medline}

Limits applied to the database:

- Type of search - Advanced search

- Population - Humans

- Age category - All Adult: 19+ years

\begin{tabular}{|c|c|c|}
\hline & SEARCH TERMS & HITS \\
\hline 1 & $\begin{array}{l}\text { (Artificial Respiration OR Respirations, Artificial OR Ventilation, Mechanical OR Mechanical Ventilations } \\
\text { OR Ventilations, Mechanical OR Mechanical Ventilation) AND (Critical IIInesses OR IIIness, Critical OR } \\
\text { IIInesses, Critical OR Critically III) AND (Skeletal muscle) }\end{array}$ & 33 \\
\hline 2 & $\begin{array}{l}\text { (Artificial Respiration OR Respirations, Artificial OR Ventilation, Mechanical OR Mechanical Ventilations } \\
\text { OR Ventilations, Mechanical OR Mechanical Ventilation) AND (Critical IIInesses OR IIIness, Critical OR } \\
\text { IIInesses, Critical OR Critically III) AND (Muscle, Respiratory OR Respiratory Muscle OR Ventilatory } \\
\text { Muscles OR Muscle, Ventilatory OR Muscles, Ventilatory OR Ventilatory Muscle) }\end{array}$ & 42 \\
\hline 3 & $\begin{array}{l}\text { (Artificial Respiration OR Respirations, Artificial OR Ventilation, Mechanical OR Mechanical Ventilations } \\
\text { OR Ventilations, Mechanical OR Mechanical Ventilation) AND (Critical IIInesses OR IIIness, Critical OR } \\
\text { IIInesses, Critical OR Critically III) AND (Diaphragm) }\end{array}$ & 38 \\
\hline 4 & $\begin{array}{l}\text { (Artificial Respiration OR Respirations, Artificial OR Ventilation, Mechanical OR Mechanical Ventilations } \\
\text { OR Ventilations, Mechanical OR Mechanical Ventilation) AND (Critical IIInesses OR IIIness, Critical OR } \\
\text { IIInesses, Critical OR Critically III ) AND (Muscle function) }\end{array}$ & 20 \\
\hline 5 & $\begin{array}{l}\text { (Artificial Respiration OR Respirations, Artificial OR Ventilation, Mechanical OR Mechanical Ventilations } \\
\text { OR Ventilations, Mechanical OR Mechanical Ventilation) AND (Critical IIInesses OR IIIness, Critical OR } \\
\text { IIInesses, Critical OR Critically III) AND (Skeletal muscle mass) }\end{array}$ & 2 \\
\hline 6 & $\begin{array}{l}\text { (Artificial Respiration OR Respirations, Artificial OR Ventilation, Mechanical OR Mechanical Ventilations } \\
\text { OR Ventilations, Mechanical OR Mechanical Ventilation) AND (Critical IIInesses OR IIIness, Critical OR } \\
\text { IIInesses, Critical OR Critically III) AND (Muscle atrophy) }\end{array}$ & 10 \\
\hline 7 & $\begin{array}{l}\text { (Critical IIInesses OR IIIness, Critical OR IIInesses, Critical OR Critically III) AND (Skeletal muscle) AND } \\
\text { (Outcome measures) }\end{array}$ & 4 \\
\hline 8 & $\begin{array}{l}\text { (Critical IIInesses OR IIIness, Critical OR IIInesses, Critical OR Critically III) AND (Skeletal muscle) AND } \\
\text { (Measurement tool or Assessment tool) }\end{array}$ & 2 \\
\hline 9 & $\begin{array}{l}\text { (Critical Illnesses OR IIIness, Critical OR Illnesses, Critical OR Critically III) AND (Artificial Respiration OR } \\
\text { Respirations, Artificial OR Ventilation, Mechanical OR Mechanical Ventilations OR Ventilations, } \\
\text { Mechanical OR Mechanical Ventilation) AND (Muscle atrophy) }\end{array}$ & 10 \\
\hline & TOTAL NUMBER OF ARTICLES & 161 \\
\hline
\end{tabular}




\section{EBSCOhost - CINAHL}

Limits applied to the database:

- Type of search - Advanced search

- Population - Human

- Age category - All Adult

\begin{tabular}{|c|c|c|}
\hline & SEARCH TERMS & HITS \\
\hline 1 & $\begin{array}{l}\text { (Critical IIInesses OR IIIness, Critical OR IIInesses, Critical OR Critically III) AND (Artificial Respiration OR } \\
\text { Artificial Respirations OR Respirations, Artificial OR Ventilation, Mechanical OR Mechanical Ventilations } \\
\text { OR Ventilations, Mechanical OR Mechanical Ventilation) AND (Skeletal muscle) }\end{array}$ & 13 \\
\hline 2 & $\begin{array}{l}\text { (Critical IIInesses OR IIIness, Critical OR IIInesses, Critical OR Critically III) AND (Artificial Respiration OR } \\
\text { Respirations, Artificial OR Ventilation, Mechanical OR Mechanical Ventilations OR Ventilations, } \\
\text { Mechanical OR Mechanical Ventilation) AND ( Muscle, Respiratory OR Respiratory Muscle OR } \\
\text { Ventilatory Muscles OR Muscle, Ventilatory OR Muscles, Ventilatory OR Ventilatory Muscle) }\end{array}$ & 14 \\
\hline 3 & $\begin{array}{l}\text { (Critical IIInesses OR IIIness, Critical OR IIInesses, Critical OR Critically III) AND (Artificial Respiration OR } \\
\text { Respirations, Artificial OR Ventilation, Mechanical OR Mechanical Ventilations OR Ventilations, } \\
\text { Mechanical OR Mechanical Ventilation) AND (Diaphragm) }\end{array}$ & 15 \\
\hline 4 & $\begin{array}{l}\text { (Critical IIInesses OR IIIness, Critical OR IIInesses, Critical OR Critically III) AND (Muscle, Respiratory OR } \\
\text { Respiratory Muscle OR Ventilatory Muscles OR Muscle, Ventilatory OR Muscles, Ventilatory OR } \\
\text { Ventilatory Muscle) AND (Measurement tool or Assessment tool) }\end{array}$ & 3 \\
\hline 5 & $\begin{array}{l}\text { (Critical IIInesses OR IIIness, Critical OR IIInesses, Critical OR Critically III) AND (Skeletal muscle) AND } \\
\text { (Measurement tool or Assessment tool) }\end{array}$ & 15 \\
\hline 6 & $\begin{array}{l}\text { (Critical IIInesses OR IIIness, Critical OR IIInesses, Critical OR Critically III) AND (Artificial Respiration OR } \\
\text { Respirations, Artificial OR Ventilation, Mechanical OR Mechanical Ventilations OR Ventilations, } \\
\text { Mechanical OR Mechanical Ventilation) AND (Muscle function) }\end{array}$ & 5 \\
\hline 7 & $\begin{array}{l}\text { (Critical IIInesses OR IIIness, Critical OR IIInesses, Critical OR Critically III) AND (Artificial Respiration OR } \\
\text { Respirations, Artificial OR Ventilation, Mechanical OR Mechanical Ventilations OR Ventilations, } \\
\text { Mechanical OR Mechanical Ventilation) AND (Skeletal muscle mass) }\end{array}$ & 2 \\
\hline 8 & $\begin{array}{l}\text { (Critical IIInesses OR IIIness, Critical OR IIInesses, Critical OR Critically III) AND (Skeletal muscle) AND } \\
\text { (Outcome measures) }\end{array}$ & 4 \\
\hline \multirow[t]{2}{*}{9} & $\begin{array}{l}\text { (Critical IIInesses OR IIIness, Critical OR IIInesses, Critical OR Critically III) AND (Diaphragm) AND } \\
\text { (Assessment tools) }\end{array}$ & 2 \\
\hline & TOTAL NUMBER OF ARTICLES & 73 \\
\hline
\end{tabular}




\section{Web of Science}

\begin{tabular}{|c|c|c|}
\hline & Search Terms & Hits \\
\hline 1 & $\begin{array}{l}\text { (Critical IIInesses; IIIness, Critical; Illnesses, Critical; Critically III) AND (Mechanical } \\
\text { Ventilation) AND (Diaphragm muscle) AND (Outcome measures) }\end{array}$ & 5 \\
\hline 2 & $\begin{array}{l}\text { (Critical IIInesses; IIIness, Critical; Illnesses, Critical; Critically III) AND (Mechanical } \\
\text { ventilation) AND (Muscle function) AND (Humans) }\end{array}$ & 7 \\
\hline 3 & $\begin{array}{l}\text { (Critical IIInesses; Illness, Critical; Illnesses, Critical; Critically III) AND (Mechanical ventilation) AND } \\
\text { (Skeletal muscle mass) AND (Humans) }\end{array}$ & 3 \\
\hline 4 & $\begin{array}{l}\text { (Critical IIInesses; Illness, Critical; Illnesses, Critical; Critically III) AND (Mechanical ventilation) AND } \\
\text { (Muscle atrophy) AND (Humans) }\end{array}$ & 3 \\
\hline 5 & $\begin{array}{l}\text { (Critical IIInesses; IIIness, Critical; IIInesses, Critical; Critically III) AND (Skeletal muscle) AND } \\
\text { (Measurement tools) }\end{array}$ & 5 \\
\hline 6 & $\begin{array}{l}\text { (Critical IIInesses; IIIness, Critical; IIInesses, Critical; Critically III) AND (Diaphragm muscle) AND } \\
\text { (Outcome measures) }\end{array}$ & 5 \\
\hline \multirow[t]{2}{*}{7} & $\begin{array}{l}\text { (Critical IIInesses; IIIness, Critical; Illnesses, Critical; Critically III) AND (Diaphragm muscle) OR } \\
\text { (Respiratory muscles) AND (Outcome measures) AND (Mechanical ventilation) AND (Humans) }\end{array}$ & 56 \\
\hline & TOTAL NUMBER OF ARTICLES & 84 \\
\hline
\end{tabular}

\section{Scopus}

\begin{tabular}{|c|l|c|}
\hline & SEARCH TERMS & HITS \\
\hline 1 & (Critical illness) AND (Mechanical ventilation) AND (Respiratory muscle) & 120 \\
\hline 2 & (Critical illness) AND (Mechanical ventilation) AND (Diaphragm) & 100 \\
\hline 3 & (Critical illness) AND (Mechanical ventilation) AND (Skeletal muscle) & 40 \\
\hline 4 & $\begin{array}{l}\text { (Critical illness) AND (Mechanical ventilation) AND (Skeletal muscle) AND (Measurement tools) OR } \\
\text { (Assessment tools) }\end{array}$ & 2 \\
\hline 5 & $\begin{array}{l}\text { (Critical illness) AND (Mechanical ventilation) AND (Respiratory muscle) AND (Measurement tools) OR } \\
\text { (Assessment tools) }\end{array}$ & 5 \\
\hline 6 & (Critical Illness) AND (Mechanical Ventilation) AND (Muscle Function) AND (Outcome measures) & 10 \\
\hline 7 & (Critical Illness) AND (Mechanical Ventilation) AND (Muscle Function) AND (Adults) & 60 \\
\hline 8 & (Critical Illness) AND (Mechanical Ventilation) AND (Muscle Wasting) & 26 \\
\hline & TOTAL NUMBER OF ARTICLES & $\mathbf{3 6 3}$ \\
\hline
\end{tabular}




\section{Science Direct}

\begin{tabular}{|c|l|c|}
\hline & Search Terms & Hits \\
\hline 1 & $\begin{array}{l}\text { (Mechanical ventilation) AND (Critical illness) AND (Skeletal muscle mass) AND (Outcome measures) } \\
\text { AND (Humans) AND (Adults) }\end{array}$ & 100 \\
\hline 2 & $\begin{array}{l}\text { (Mechanical ventilation) AND (Critical illness) AND (Muscle atrophy) AND (Outcome measures) AND } \\
\text { (Humans) AND (Adults) }\end{array}$ & 104 \\
\hline 3 & $\begin{array}{l}\text { (Mechanical ventilation) AND (Critical illness) AND (Diaphragm) AND (Outcome measures) AND } \\
\text { (Humans) AND (Adults) }\end{array}$ & 111 \\
\hline & TOTAL NUMBER OF ARTICLES & $\mathbf{3 1 5}$ \\
\hline
\end{tabular}

\section{Pubmed}

Limits applied to the database:

- Population - Humans

- Age category - Adult: 19+ years

\begin{tabular}{|c|c|c|}
\hline & SEARCH TERMS & HITS \\
\hline 1 & $\begin{array}{l}\text { ("Critical illness" [Title/Abstract] OR "Critical illness" [MeSH Terms]) AND ("Respiration, Artificial" } \\
\text { [Title/Abstract] OR "Respiration, Artificial" [MeSH Terms]) AND("Muscle, Skeletal"[Title] OR "Muscle, } \\
\text { Skeletal"[MeSH Terms]) }\end{array}$ & 53 \\
\hline 2 & $\begin{array}{l}\text { ("Critical illness" [Title/Abstract] OR "Critical illness" [MeSH Terms]) AND ("Respiration, Artificial" } \\
\text { [Title/Abstract] OR "Respiration, Artificial" [MeSH Terms]) AND (Diaphragm muscle) }\end{array}$ & 25 \\
\hline 3 & $\begin{array}{l}\text { ("Critical illness" [Title/Abstract] OR "Critical illness" [MeSH Terms]) AND ("Respiration, Artificial" } \\
\text { [Title/Abstract] OR "Respiration, Artificial" [MeSH Terms]) AND (Muscle Function) }\end{array}$ & 80 \\
\hline 4 & ("Critical illness" [Title/Abstract] OR "Critical illness" [MeSH Terms]) AND (Skeletal muscle mass) & 36 \\
\hline 5 & $\begin{array}{l}\text { ("Critical illness" [Title/Abstract] OR "Critical illness" [MeSH Terms]) AND ("Respiration, Artificial" } \\
\text { [Title/Abstract] OR "Respiration, Artificial" [MeSH Terms]) AND (Muscle structure) }\end{array}$ & 3 \\
\hline 6 & $\begin{array}{l}\text { ("Critical illness" [Title/Abstract] OR "Critical illness" [MeSH Terms]) AND ("Respiration, Artificial" } \\
\text { [Title/Abstract] OR "Respiration, Artificial" [MeSH Terms]) AND (Assessment tool) }\end{array}$ & 35 \\
\hline 7 & $\begin{array}{l}\text { ("Critical illness" [Title/Abstract] OR "Critical illness" [MeSH Terms]) AND (Muscle function) AND } \\
\text { (Assessment tool) }\end{array}$ & 6 \\
\hline 8 & $\begin{array}{l}\text { ("Critical illness" [Title/Abstract] OR "Critical illness" [MeSH Terms]) AND ("Respiration, Artificial" } \\
\text { [Title/Abstract] OR "Respiration, Artificial" [MeSH Terms]) AND (Muscle endurance) }\end{array}$ & 3 \\
\hline 9 & $\begin{array}{l}\text { ("Critical illness" [Title/Abstract] OR "Critical illness" [MeSH Terms]) AND ("Muscle, Skeletal"[Title] OR } \\
\text { "Muscle, Skeletal"[MeSH Terms]) AND (Assessment tools) }\end{array}$ & 5 \\
\hline 10 & $\begin{array}{l}(\text { ("Critical illness" [Title/Abstract] OR "Critical illness" [MeSH Terms]) AND ("Respiration, Artificial" } \\
\text { [Title/Abstract] OR "Respiration, Artificial" [MeSH Terms]) AND (Muscle wasting) }\end{array}$ & 18 \\
\hline 11 & $\begin{array}{l}\text { ("Critical illness" [Title/Abstract] OR "Critical illness" [MeSH Terms]) AND ("Respiration, Artificial" } \\
\text { [Title/Abstract] OR "Respiration, Artificial" [MeSH Terms]) AND (Muscle Function) AND (Outcome } \\
\text { Measures) }\end{array}$ & 22 \\
\hline & TOTAL NUMBER OF ARTICLES & 286 \\
\hline
\end{tabular}




\section{Addendum B: Year of Publication}

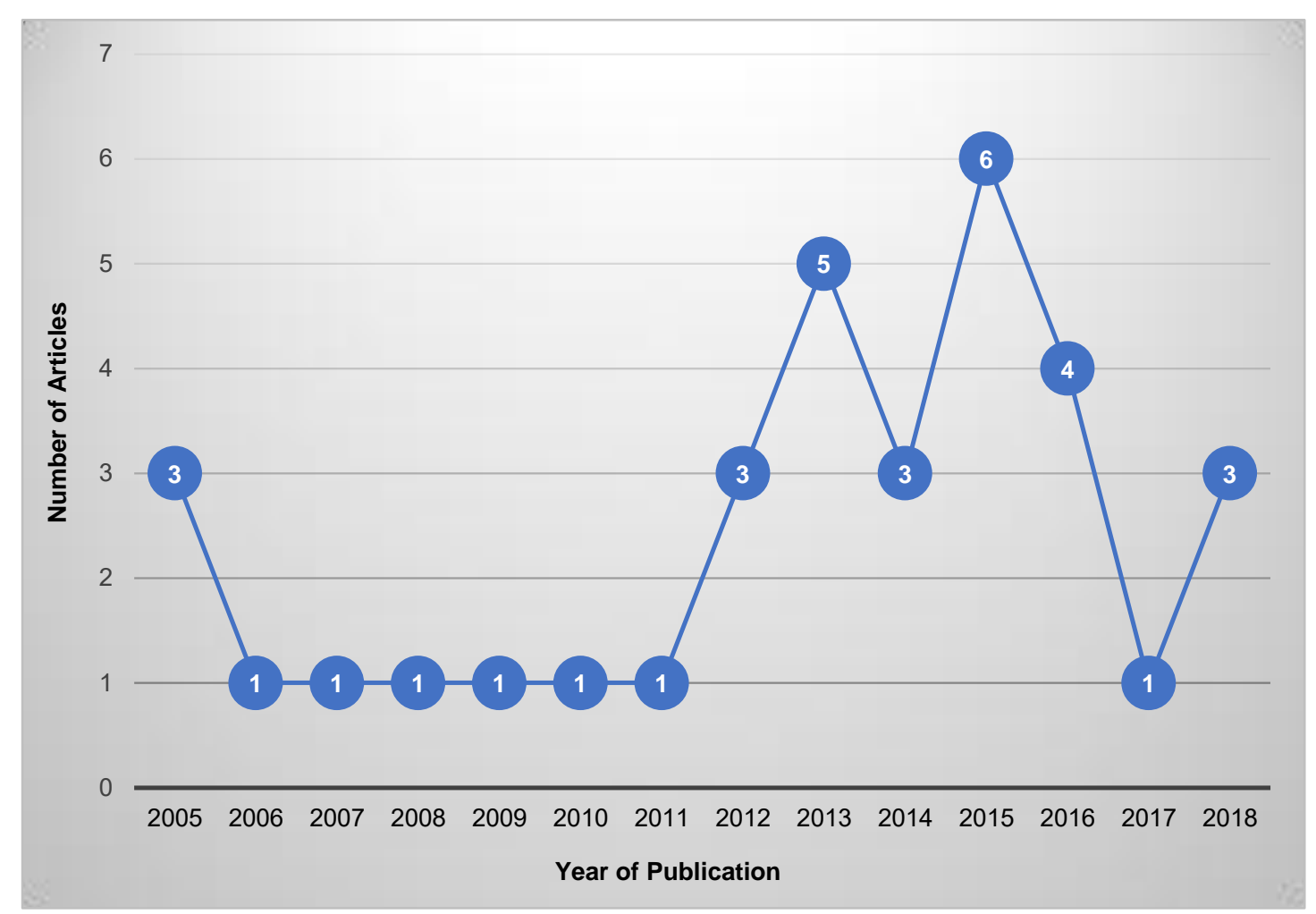

Figure 1: Number of articles published per year 
Addendum C: Study Population Diagnosis

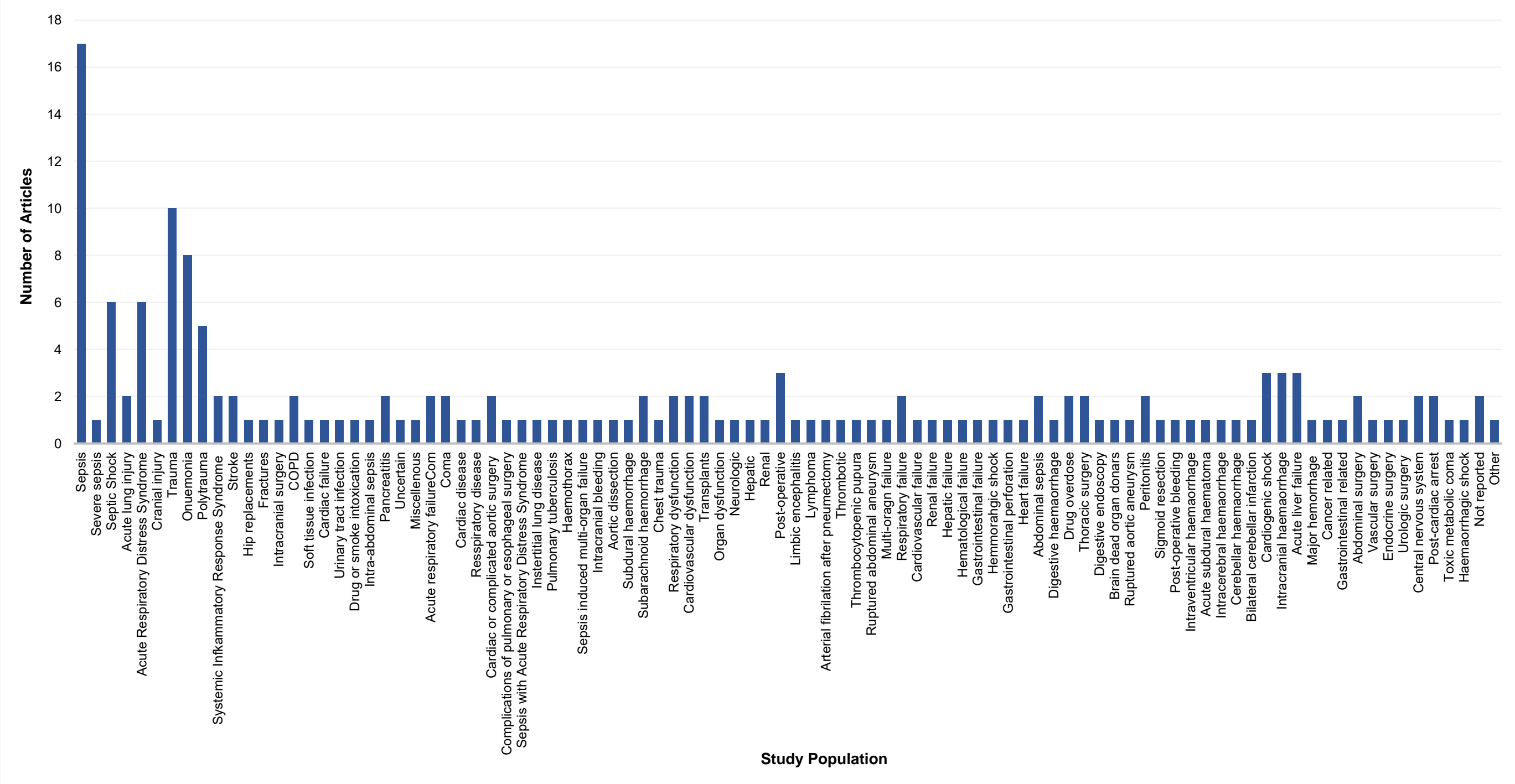

Figure 2: Study population diagnosis 
Addendum D: Muscle Studied per Measurement Tool

\begin{tabular}{|c|c|c|c|c|c|}
\hline \multicolumn{6}{|c|}{ Muscle Structure } \\
\hline \multicolumn{2}{|c|}{ Muscle biopsy } & \multicolumn{2}{|c|}{ Ultrasonography } & \multicolumn{2}{|c|}{ Computed tomography } \\
\hline Author(s) & Type of Skeletal Muscle & Author(s) & Type of Skeletal Muscle & Author(s) & Type of Skeletal Muscle \\
\hline Bierbrauer et al. (10) & Vastus lateralis & Baldwin and Bersten (5) & Right hemi-diaphragm & Lee et al. (70) & Diaphragm \\
\hline Derde et al. (33) & $\begin{array}{l}\text { Vastus lateralis, Rectus } \\
\text { abdominus }\end{array}$ & Cartwright et al. (16) & $\begin{array}{l}\text { Diaphragm, Abductor digiti minimi, } \\
\text { Biceps brachii, Brachialis, Tibialis } \\
\text { anterior, Vastus lateralis }\end{array}$ & Sheean et al. (96) & $\begin{array}{l}\text { Rectus abdominus, Transverse } \\
\text { abdominus, Erector spinae, Quadratus } \\
\text { lumborum, Internal \& External } \\
\text { obliques }\end{array}$ \\
\hline Fredriksson et al. & $\begin{array}{l}\text { Intercostal muscles, Vastus } \\
\text { lateralis }\end{array}$ & Goligher et al. (50) & Right hemi-diaphragm & & \\
\hline Hooijman et al. (44) & Diaphragm & Goligher et al. (51) & Right hemi-diaphragm & & \\
\hline Jaber et al. (64) & Diaphragm & Hernandez-Scorro et al. (57) & Rectus femoris & & \\
\hline Klaude et al. (66) & Vastus lateralis & Llano-diez et al. (72) & Tibialis anterior & & \\
\hline Llano-diez et al. (72) & Tibialis anterior & Puthucheary et al. (84) & Rectus femoris & & \\
\hline Puthucheary et al. (84) & Vastus lateralis & Puthucheary et al. (85) & Rectus femoris & & \\
\hline Puthucheary et al. (85) & Vastus lateralis & Schepens et al. (90) & $\begin{array}{l}\text { Right hemi-diaphragm } \\
\text { Elbow flexor compartment, Vastus }\end{array}$ & & \\
\hline Puthucheary et al. (87) & Vastus lateralis & Turton et al. (99) & $\begin{array}{l}\text { lateralis, Medial head of the } \\
\text { gastrocnemius }\end{array}$ & & \\
\hline Van den berg et al. (101) & Diaphragm & Umbrello et al. (100) & Diaphragm & & \\
\hline Weber-Carstens et al. (106) & Vastus lateralis & Zambon et al. (110) & Diaphragm & & \\
\hline \multicolumn{6}{|c|}{ Muscle Function } \\
\hline \multicolumn{2}{|c|}{ Electrophysiological measurements } & \multicolumn{2}{|c|}{ Muscle torque via nerve stimulation } & \multicolumn{2}{|r|}{ Dynamometry } \\
\hline Author(s) & Type of Skeletal Muscle & Author(s) & Type of Skeletal Muscle & Author(s) & Type of Skeletal Muscle \\
\hline Bierbrauer et al. (10) & $\begin{array}{l}\text { Tibialis anterior, Extensor } \\
\text { digitorum communis }\end{array}$ & Ginz et al. (47) & $\begin{array}{l}\text { Tibialis anterior, Extensor hallucis } \\
\text { longus, Extensor digitorum longus, } \\
\text { Peroneus tertius }\end{array}$ & $\begin{array}{l}\text { Baldwin and Bersten } \\
\text { (5) }\end{array}$ & Elbow flexors, Knee extensors \\
\hline Llano-diez et al. (72) & $\begin{array}{l}\text { Tibilias anterior, vastus } \\
\text { lateralis }\end{array}$ & Ginz et al. (48) & $\begin{array}{l}\text { Tibialis anterior, Extensor hallucis } \\
\text { longus, Extensor digitorum longus, } \\
\text { Peroneus tertius }\end{array}$ & & \\
\hline Weber-Carstens et al. (105) & $\begin{array}{l}\text { Abductor pollicis brevis, } \\
\text { Tibialis anterior, Extensor } \\
\text { digitorum brevis }\end{array}$ & & & & \\
\hline Weber-Carstens et al. (106) & Quadriceps & & & & \\
\hline
\end{tabular}

Figure 3: Muscles studied per measurement tools 


\section{Addendum E: Testing Procedure of the Measurement Tool}

Table 1: Summary of the ultrasonography methodology used in the studies measuring respiratory and peripheral muscle structure

\begin{tabular}{|c|c|c|c|c|c|c|c|c|c|c|c|}
\hline \multirow[b]{2}{*}{ Author(s) } & \multirow{2}{*}{$\begin{array}{l}\text { Name of } \\
\text { muscles }\end{array}$} & \multirow{2}{*}{ Name of device } & \multicolumn{2}{|c|}{ Transducer } & \multirow{2}{*}{$\begin{array}{l}\text { Frequency } \\
\quad(\mathrm{MHz})\end{array}$} & \multicolumn{2}{|c|}{ US mode } & \multirow{2}{*}{ Positioning } & \multicolumn{3}{|c|}{ Probe placement } \\
\hline & & & $\begin{array}{l}\text { Convex- } \\
\text { phased }\end{array}$ & $\begin{array}{l}\text { Linear- } \\
\text { array }\end{array}$ & & B-mode & M-mode & & $\begin{array}{l}\text { Intercostal } \\
\text { space }\end{array}$ & ZOA & Axillary line \\
\hline \multicolumn{12}{|c|}{ Respiratory } \\
\hline $\begin{array}{l}\text { Baldwin and } \\
\text { Bersten (5) }\end{array}$ & $\begin{array}{l}\text { Right hemi- } \\
\text { diaphragm }\end{array}$ & NR & $N R$ & $\checkmark$ & NR & NR & NR & $45^{\circ}$ heads up & 9th & $\checkmark$ & Mid-axillary \\
\hline $\begin{array}{l}\text { Cartwright et } \\
\text { al. (16) }\end{array}$ & NR & $\begin{array}{c}\text { Portable } \\
\text { ultrasound } \\
\text { device (Esaote } \\
\text { Biosound MyLab } \\
25 ; \text { Esaote } \\
\text { Group, Genoa, } \\
\text { Italy) }\end{array}$ & NR & $\checkmark$ & 18 & NR & NR & NR & 8th & NR & Anterior axillary \\
\hline $\begin{array}{l}\text { Goligher et } \\
\text { al. (50) }\end{array}$ & $\begin{array}{l}\text { Right hemi- } \\
\text { diaphragm }\end{array}$ & NR & $N R$ & $\checkmark$ & 13 & NR & NR & NR & 9th or 10 th & $\checkmark$ & $\begin{array}{l}\text { Between anterior and } \\
\text { mid-axillary }\end{array}$ \\
\hline $\begin{array}{l}\text { Goligher et } \\
\text { al. (51) }\end{array}$ & $\begin{array}{l}\text { Right hemi- } \\
\text { diaphragm }\end{array}$ & NR & $N R$ & $\checkmark$ & 13 & NR & NR & NR & 9th or 10 th & $\checkmark$ & $\begin{array}{l}\text { Between anterior and } \\
\text { mid-axillary }\end{array}$ \\
\hline $\begin{array}{l}\text { Schepens et } \\
\text { al. (90) }\end{array}$ & $\begin{array}{l}\text { Right hemi- } \\
\text { diaphragm }\end{array}$ & NR & NR & $\checkmark$ & 13 & $\checkmark$ & NR & $30^{\circ}-45^{\circ}$ heads up & 8th or 9th & NR & $\begin{array}{l}\text { Mid-axillary or Mid- } \\
\text { clavicular }\end{array}$ \\
\hline $\begin{array}{l}\text { Umbrello et } \\
\text { al. (100) }\end{array}$ & NR & $\begin{array}{l}\text { LogiQ7 (GE } \\
\text { Healthcare, Little } \\
\text { Chalfont, UK) }\end{array}$ & Y & $\checkmark$ & $7.5 \& 10$ & NR & $\checkmark$ & NR & 10th & $\checkmark$ & Mid-axillary \\
\hline $\begin{array}{l}\text { Zambon et } \\
\text { al. (110) }\end{array}$ & NR & $\begin{array}{l}\text { LOGIQ e (GE, } \\
\text { Fairfield, CT) } \\
\text { ultrasound } \\
\text { portable system }\end{array}$ & $N R$ & $\checkmark$ & $7 \& 13$ & $\checkmark$ & NR & $30^{\circ}$ heads up & 10th & $\checkmark$ & Mid-axillary \\
\hline \multicolumn{12}{|c|}{ Peripheral } \\
\hline $\begin{array}{l}\text { Baldwin and } \\
\text { Bersten (5) }\end{array}$ & NR & NR & NR & $\checkmark$ & NR & NR & NR & Supine & \multicolumn{3}{|c|}{ NR } \\
\hline $\begin{array}{l}\text { Cartwright et } \\
\text { al. (16) }\end{array}$ & $\begin{array}{c}\text { Abductor digiti } \\
\text { minimi, Biceps } \\
\text { brachii, } \\
\text { Brachialis, } \\
\text { Vastus lateralis, } \\
\text { Tibialis anterior }\end{array}$ & $\begin{array}{c}\text { Portable } \\
\text { ultrasound } \\
\text { device (Esaote } \\
\text { Biosound MyLab } \\
\text { 25;Esaote } \\
\text { Group, Genoa, } \\
\text { Italy) }\end{array}$ & NR & $\checkmark$ & 18 & NR & NR & NR & \multicolumn{3}{|c|}{$\begin{array}{l}\text { UL: Middle of the } 5 \text { th metacarpal, } 10 \mathrm{~cm} \text { to the } \\
\text { antecubital fossa } \\
\text { LL: } 5 \mathrm{~cm} \text { distal to the fibular head, } 15 \mathrm{~cm} \text { proximal to } \\
\text { the superior portion of the patella }\end{array}$} \\
\hline
\end{tabular}


Table 1. continued

\begin{tabular}{|c|c|c|c|c|c|c|c|c|c|c|c|}
\hline \multirow[b]{2}{*}{ Author(s) } & \multirow{2}{*}{$\begin{array}{l}\text { Name of } \\
\text { muscles }\end{array}$} & \multirow[b]{2}{*}{ Name of device } & \multicolumn{2}{|c|}{ Transducer } & \multirow{2}{*}{$\begin{array}{l}\text { Frequency } \\
\quad(\mathrm{MHz})\end{array}$} & \multicolumn{2}{|c|}{ US mode } & \multirow{2}{*}{ Positioning } & \multicolumn{3}{|c|}{ Probe placement } \\
\hline & & & $\begin{array}{l}\text { Convex- } \\
\text { phased }\end{array}$ & $\begin{array}{l}\text { Linear- } \\
\text { array }\end{array}$ & & B-mode & M-mode & & $\begin{array}{l}\text { Intercostal } \\
\text { space }\end{array}$ & ZOA & Axillary line \\
\hline \multicolumn{12}{|c|}{ Respiratory } \\
\hline $\begin{array}{l}\text { Hernandez- } \\
\text { Scorro et al. } \\
\quad(57)\end{array}$ & Rectus femoris & $\begin{array}{l}\text { Aplio } 500 \\
\text { ultrasound } \\
\text { system }\end{array}$ & NR & $\checkmark$ & $10 \& 12$ & $\checkmark$ & NR & $\begin{array}{l}\text { Supine, knees } \\
\text { extended and } \\
\text { relaxed to full } \\
\text { extension }\end{array}$ & \multicolumn{3}{|c|}{ LL: Two -thirds along the femur length } \\
\hline $\begin{array}{l}\text { Llano-diez et } \\
\quad \text { al. (72) }\end{array}$ & $\begin{array}{l}\text { Vastus } \\
\text { lateralis, } \\
\text { Tibialis anterior }\end{array}$ & $\begin{array}{c}\text { Siemens Acuson } \\
\text { Antares } \\
\text { Ultrasound } \\
\text { System, } \\
\text { Mountain View, } \\
\text { CA, USA }\end{array}$ & NR & $\checkmark$ & 9.4 & NR & NR & NR & \multicolumn{3}{|c|}{$\begin{array}{l}\text { LL: } 50 \%, 40 \% \text { and } 30 \% \text { of the distance from the } \\
\text { proximal part of the fibula head to the distal tip of } \\
\text { the lateral malleolus }\end{array}$} \\
\hline $\begin{array}{l}\text { Puthucheary } \\
\text { et al. (84) }\end{array}$ & Rectus femoris & NR & NR & NR & NR & $\checkmark$ & NR & NR & \multicolumn{3}{|c|}{ NR } \\
\hline $\begin{array}{l}\text { Puthucheary } \\
\text { et al. (85) }\end{array}$ & $\begin{array}{c}\text { Vastus } \\
\text { lateralis, } \\
\text { Rectus femoris }\end{array}$ & NR & NR & NR & NR & $\checkmark$ & NR & NR & \multicolumn{3}{|c|}{ NR } \\
\hline \multirow{3}{*}{$\begin{array}{l}\text { Turton et al. } \\
\quad(99)\end{array}$} & \multirow{3}{*}{$\begin{array}{l}\text { Elbow flexor } \\
\text { compartment, } \\
\text { Vastus } \\
\text { lateralis, } \\
\text { Gastrocnemius }\end{array}$} & \multirow{3}{*}{ NR } & \multirow{3}{*}{ NR } & \multirow{3}{*}{$\checkmark$} & \multirow{3}{*}{ NR } & \multirow{3}{*}{$\checkmark$} & \multirow{3}{*}{ NR } & \multirow{3}{*}{$\begin{array}{l}\text { Anatomical position } \\
\text { VL: Limb flat to the } \\
\text { bed G: Flexion of } \\
\text { the Knee }\end{array}$} & \multicolumn{3}{|c|}{$\begin{array}{l}\text { UL: } 5 \mathrm{~cm} \text { proximal to the line connecting both the } \\
\text { medial and lateral epicondyles above the base of } \\
\text { the antecubital triangle }\end{array}$} \\
\hline & & & & & & & & & \multicolumn{3}{|c|}{$\begin{array}{l}\text { LL -VL: } 10 \mathrm{~cm} \text { proximal to the lateral condyle of the } \\
\text { femur. }\end{array}$} \\
\hline & & & & & & & & & \multicolumn{3}{|c|}{ G: Widest point of the muscle belly } \\
\hline
\end{tabular}


Table 2: Summary of the methodology for muscle biopsy of the respiratory muscles

\begin{tabular}{|c|c|c|c|}
\hline Author(s) & Name of muscles & Procedure & Storage method \\
\hline Fredriksson et al. (44) & Intercostal & $\begin{array}{l}\text { Obtained from the fifth and sixth rib during surgery using a Bergstrom } \\
\text { biopsy needle }\end{array}$ & $\begin{array}{l}\text { Weighed and frozen in liquid nitrogen and stored at }-80^{\circ} \\
\text { Celsius until analysis }\end{array}$ \\
\hline Jaber et al. (64) & $(\mathrm{R})$ hemi-diaphragm & $\begin{array}{l}\text { Obtained from the right hemi-diaphragm, at the zone of apposition (ZOA), } \\
\text { in the mid-axillary line. }\end{array}$ & NR \\
\hline Hooijman et al. (60) & Diaphragm & Obtained via surgery and cut into smaller sections & $\begin{array}{l}\text { Frozen in liquid nitrogen and stored at }-80^{\circ} \text { Celsius until } \\
\text { analysis }\end{array}$ \\
\hline $\begin{array}{c}\text { Van den Berg et al. } \\
\text { (101) }\end{array}$ & Diaphragm & Obtained via surgery and cut into smaller sections & $\begin{array}{l}\text { Frozen in liquid nitrogen and stored at }-80^{\circ} \text { Celsius until } \\
\text { analysis }\end{array}$ \\
\hline
\end{tabular}


Table 3: Summary of the methodology for muscle biopsy of the peripheral muscles

\begin{tabular}{|c|c|c|c|}
\hline Author(s) & Name of muscles & Procedure & Storage method \\
\hline Bierbrauer et al. (10) & Vastus lateralis & NR & NR \\
\hline Derde et al. (33) & $\begin{array}{l}\text { Vastus lateralis, Rectus } \\
\text { abdominus }\end{array}$ & NR & $\begin{array}{l}\text { Frozen in liquid nitrogen and stored at }-80^{\circ} \text { Celsius } \\
\text { until analysis }\end{array}$ \\
\hline $\begin{array}{l}\text { Fredriksson et al. } \\
\qquad(44)\end{array}$ & Vastus lateralis & Obtained using a Bergstrom biopsy needle & $\begin{array}{l}\text { Frozen in liquid nitrogen and stored at }-80^{\circ} \text { Celsius } \\
\text { until analysis }\end{array}$ \\
\hline Hooijman et al. (60) & NR & Obtained via surgery and cut into smaller sections & $\begin{array}{l}\text { Frozen in liquid nitrogen and stored at }-80^{\circ} \text { Celsius } \\
\text { until analysis }\end{array}$ \\
\hline Klaude et al. (66) & Vastus lateralis & NR & $\begin{array}{l}\text { Frozen in liquid nitrogen and stored at }-80^{\circ} \text { Celsius } \\
\text { until analysis }\end{array}$ \\
\hline Llano-diez et al. (72) & Tibialis anterior & $\begin{array}{l}\text { A 3-5mm skin incision, penetrating the fascia with a scalpel blade and then } \\
\text { inserting the conchotome instrument through the incision and into the muscle to } \\
\text { dissect and obtain the muscle }\end{array}$ & $\begin{array}{l}\text { Frozen in liquid nitrogen and stored at }-80^{\circ} \text { Celsius } \\
\text { until analysis }\end{array}$ \\
\hline $\begin{array}{l}\text { Puthucheary et al. } \\
\qquad(84)\end{array}$ & Vastus lateralis & $\begin{array}{l}\text { A 3-5mm skin incision, penetrating the fascia with a scalpel blade and then } \\
\text { inserting the conchotome instrument through the incision and into the muscle to } \\
\text { dissect and obtain the muscle }\end{array}$ & $\begin{array}{l}\text { Frozen in liquid nitrogen and stored at }-80^{\circ} \text { Celsius } \\
\text { until analysis }\end{array}$ \\
\hline $\begin{array}{l}\text { Puthucheary et al. } \\
\text { (85) }\end{array}$ & Vastus lateralis & I & $\begin{array}{l}\text { Frozen in liquid nitrogen and stored at }-80^{\circ} \text { Celsius } \\
\text { until analysis }\end{array}$ \\
\hline $\begin{array}{l}\text { Puthucheary et al. } \\
\text { (87) }\end{array}$ & Vastus lateralis & NR & NR \\
\hline $\begin{array}{l}\text { Weber-Carstens et } \\
\text { al. (106) }\end{array}$ & Vastus lateralis & NR & $\begin{array}{l}\text { Frozen in liquid nitrogen and stored at }-80^{\circ} \text { Celsius } \\
\text { until analysis }\end{array}$ \\
\hline $\begin{array}{l}\text { Wollersheim et al. } \\
\text { (109) }\end{array}$ & Vastus lateralis & NR & NR \\
\hline
\end{tabular}


Table 4: Methodology of CT Scan of the respiratory and peripheral muscles

\begin{tabular}{|c|c|c|c|c|}
\hline Author(s) & Name of muscle & Software & Procedure & $\begin{array}{c}\text { Number of } \\
\text { measurements }\end{array}$ \\
\hline \multicolumn{5}{|c|}{ Respiratory } \\
\hline Lee et al. (70) & Diaphragm & $\begin{array}{c}\text { 3D Imaging software } \\
\text { (Rapidia 2.8; Infinitt, Seoul, } \\
\text { Korea) }\end{array}$ & $\begin{array}{l}\text { The 3D imaging software automatically displayed corresponding points on the right and } \\
\text { left sides of the diaphragm (coronal and axial images) after which, dot cursors were } \\
\text { placed on the points where the lines from both sides of the diaphragm intersected. } \\
\text { Diaphragm thickness was then measured at the same points on axial and coronal } \\
\text { images using the cursor. }\end{array}$ & 3 \\
\hline \multicolumn{5}{|c|}{ Postural } \\
\hline Sheean et al. (96) & $\begin{array}{l}\text { Rectus abdominus, Transverse } \\
\text { abdominus, Erector spinae, Quadratus } \\
\text { lumborum, Internal and External obliques }\end{array}$ & $\begin{array}{l}\text { Mimics software (version } \\
14, \text { Materialise, Leuven, } \\
\text { Belgium) }\end{array}$ & $\begin{array}{l}\text { A single transverse CT slice was used to identify the postural muscles within the L3 } \\
\text { region. Once the muscles were identified, images were captured and analysed using } \\
\text { the Mimics software, and the cross-sectional area was calculated. }\end{array}$ & 3 \\
\hline
\end{tabular}

Table 5: Bilateral phrenic nerve stimulation methodology used in the studies during diaphragm assessment

\begin{tabular}{|c|c|c|c|c|c|c|c|c|c|c|c|}
\hline \multirow{2}{*}{ Author(s) } & \multirow{2}{*}{$\begin{array}{l}\text { Name of the } \\
\text { magnetic } \\
\text { coils }\end{array}$} & \multirow{2}{*}{$\begin{array}{c}\text { Positioning } \\
\begin{array}{c}\text { Semi- } \\
\text { recumbent }\end{array}\end{array}$} & \multicolumn{2}{|c|}{ Gastric Pressure } & \multicolumn{2}{|c|}{$\begin{array}{l}\text { Oesophageal } \\
\text { Pressure }\end{array}$} & \multicolumn{2}{|c|}{ Endotracheal Pressure } & \multicolumn{3}{|c|}{ Stimuli } \\
\hline & & & $\begin{array}{l}\text { Type of } \\
\text { catheter }\end{array}$ & $\begin{array}{l}\text { Volume of } \\
\text { catheter }\end{array}$ & $\begin{array}{l}\text { Type of } \\
\text { catheter }\end{array}$ & $\begin{array}{l}\text { Volume of } \\
\text { catheter }\end{array}$ & Name of device & $\begin{array}{l}\text { Procedure } \\
\text { reported }\end{array}$ & Measurements & Intensity & Location \\
\hline $\begin{array}{c}\text { Buscher et al. } \\
\text { (15) }\end{array}$ & $\begin{array}{l}\text { Magstim } 200 \\
\text { stimulator }\end{array}$ & (2) & Balloon & $0.5 \mathrm{ml}$ & Balloon & $1.5 \mathrm{ml}$ & NR & NR & $8 \& 10$ & $100 \%$ & $\begin{array}{l}\text { Over the cervical spine at } \\
\text { the level of } \mathrm{C} 5-\mathrm{C} 7\end{array}$ \\
\hline $\begin{array}{c}\text { Demoule et al. } \\
\text { (31) }\end{array}$ & $\begin{array}{l}\text { Magstim } 200 \\
\text { stimulator }\end{array}$ & $\checkmark$ & NR & NR & NR & NR & $\begin{array}{c}\text { Differential pressure } \\
\text { transducer (Huba } \\
\text { Control, Würenlos, } \\
\text { Switzerland) } \\
\end{array}$ & $\checkmark$ & 3 & $100 \%$ & $\begin{array}{c}\text { Posterior to the } \\
\text { sternocleidomastoid muscle } \\
\text { at the level of the cricoid } \\
\text { cartilage }\end{array}$ \\
\hline $\begin{array}{c}\text { Demoule et al. } \\
\text { (32) }\end{array}$ & $\begin{array}{l}\text { Magstim } 200 \\
\text { stimulator }\end{array}$ & $\checkmark$ & NR & NR & NR & NR & NR & $\checkmark$ & 3 & $100 \%$ & $\begin{array}{c}\text { Posterior to the } \\
\text { sternocleidomastoid muscle } \\
\text { at the level of the cricoid } \\
\text { cartilage }\end{array}$ \\
\hline $\begin{array}{c}\text { Hermans et al. } \\
(54)\end{array}$ & $\begin{array}{l}\text { Magstim } 200 \\
\text { stimulator }\end{array}$ & $\checkmark$ & Balloon & $2 \mathrm{ml}$ & Balloon & $0.5 \mathrm{ml}$ & NR & NR & 3 & $100 \%$ & $\begin{array}{c}\text { Posterior to the } \\
\text { sternocleidomastoid muscle } \\
\text { at the level of the cricoid } \\
\text { cartilage }\end{array}$ \\
\hline Jaber et al. (64) & $\begin{array}{l}\text { Magstim } 200 \\
\text { stimulator }\end{array}$ & $\checkmark$ & NR & NR & NR & NR & NR & $\checkmark$ & 3 & NR & $\begin{array}{c}\text { Posterior to the } \\
\text { sternocleidomastoid muscle } \\
\text { at the level of the cricoid } \\
\text { cartilage }\end{array}$ \\
\hline $\begin{array}{l}\text { Supinski and } \\
\text { Callahan (98) }\end{array}$ & $\begin{array}{l}\text { Magstim } 200 \\
\text { stimulator }\end{array}$ & $\checkmark$ & $\begin{array}{l}\text { Balloon- } \\
\text { tipped }\end{array}$ & NR & $\begin{array}{l}\text { Balloon- } \\
\text { tipped }\end{array}$ & NR & NR & NR & 3 & $100 \%$ & $\begin{array}{c}\text { Adjacent to the border of } \\
\text { the sternocleidomastoid } \\
\text { muscle }\end{array}$ \\
\hline $\begin{array}{l}\text { Umbrello et al. } \\
\text { (100) }\end{array}$ & NR & NR & $\begin{array}{l}\text { Double } \\
\text { balloon }\end{array}$ & $2 \mathrm{ml}$ & $\begin{array}{l}\text { Double } \\
\text { balloon }\end{array}$ & $2 \mathrm{ml}$ & NR & NR & NR & NR & NR \\
\hline
\end{tabular}

KEYS: $\checkmark$ - Yes; NR - Not Reported 
Table 6: Summary of the methodology for MIP and MEP

\begin{tabular}{|c|c|c|c|c|c|c|}
\hline Author(s) & MIP & MEP & Name of device & Positioning & Procedure reported & $\begin{array}{l}\text { Number of } \\
\text { measurements taken }\end{array}$ \\
\hline $\begin{array}{l}\text { Baldwin and Bersten } \\
\text { (5) }\end{array}$ & $\checkmark$ & NR & $\begin{array}{l}\text { MicroRPM Respiratory } \\
\text { Pressure Meter }\end{array}$ & Semi-recumbent & $\begin{array}{l}\text { Patients inspired maximally from residual volume through a } \\
\text { mouthpiece and sustained the effort for at least one second. }\end{array}$ & Best effort \\
\hline Bissett (11) & $\checkmark$ & NR & $\begin{array}{l}\text { MicroRPM Respiratory } \\
\text { Pressure Meter }\end{array}$ & Semi-recumbent & $\begin{array}{l}\text { Patients inspired maximally from residual volume through a } \\
\text { mouthpiece and sustained the effort for at least one second. }\end{array}$ & 3 \\
\hline De Jonghe et al. (29) & $\checkmark$ & $\checkmark$ & Tracheal tubing & Semi-recumbent & $\begin{array}{l}\text { MIP was measured after a forced expiration. } \\
\text { MEP was measured after a forced inspiration against a manual } \\
\text { occlusion and was held for more than one second. }\end{array}$ & 3 \\
\hline
\end{tabular}

KEYS: $\checkmark$ - Yes; NR - Not Reported 
Table 7: Summary of the methodology for muscle torque via nerve stimulation

\begin{tabular}{|c|c|c|c|c|c|c|c|c|c|c|}
\hline \multirow{2}{*}{ Author(s) } & \multirow{2}{*}{$\begin{array}{l}\text { Name of } \\
\text { muscles }\end{array}$} & \multirow{2}{*}{ Nerve } & \multirow{2}{*}{$\begin{array}{c}\text { Position } \\
\text { of the } \\
\text { patient }\end{array}$} & \multirow{2}{*}{$\begin{array}{c}\text { Name of } \\
\text { the } \\
\text { stimulator }\end{array}$} & \multicolumn{2}{|c|}{ Electrodes } & \multirow{2}{*}{ Procedure } & \multicolumn{3}{|c|}{ Stimulation } \\
\hline & & & & & Type & Placement & & Voltage & Current & Time \\
\hline $\begin{array}{c}\text { Ginz et al. } \\
(47)\end{array}$ & $\begin{array}{c}\text { Tibialis anterior, } \\
\text { Extensor hallucis } \\
\text { longus, } \\
\text { Extensor } \\
\text { digitorum longus, } \\
\text { Peroneus tertius }\end{array}$ & $\begin{array}{l}\text { Common } \\
\text { peroneal }\end{array}$ & Supine & $\begin{array}{c}\text { S11, Grass } \\
\text { Medical } \\
\text { Instruments, } \\
\text { Quincy, MA, } \\
\text { USA }\end{array}$ & $\begin{array}{l}\text { Small } \\
\text { ball- } \\
\text { shaped }\end{array}$ & $\begin{array}{l}\text { Behind the } \\
\text { fibula's } \\
\text { head }\end{array}$ & $\begin{array}{l}\text { The electrodes were placed against the skin behind the } \\
\text { fibula's head to deliver stimulation to the common } \\
\text { peroneal nerve. The foot of the leg was strapped to a } \\
\text { muscle force assessment system and was secured into a } \\
\text { boot which was fixed to a torque plate. The torque plate } \\
\text { was used to determine the optimal muscle length for } \\
\text { maximum isometric contraction by moving it until twitch } \\
\text { torques were at their maximal levels. Once the optimal } \\
\text { muscle length was determined, the isometric contraction } \\
\text { force was recorded. }\end{array}$ & $50-90 \mathrm{~V}$ & $40-90 \mathrm{~mA}$ & $\begin{array}{l}0.3 \\
\mathrm{~ms}\end{array}$ \\
\hline $\begin{array}{c}\text { Ginz et al. } \\
(48)\end{array}$ & $\begin{array}{c}\text { Tibialis anterior, } \\
\text { Extensor hallucis } \\
\text { longus, } \\
\text { Extensor } \\
\text { digitorum longus, } \\
\text { Peroneus tertius }\end{array}$ & $\begin{array}{l}\text { Common } \\
\text { peroneal }\end{array}$ & NR & $\begin{array}{c}\text { S11, Grass } \\
\text { Medical } \\
\text { Instruments, } \\
\text { Quincy, MA, } \\
\text { USA }\end{array}$ & $\begin{array}{c}\text { Small } \\
\text { ball- } \\
\text { shaped }\end{array}$ & $\begin{array}{c}\text { Behind the } \\
\text { fibula's } \\
\text { head }\end{array}$ & $\begin{array}{l}\text { The electrodes were placed against the skin behind the } \\
\text { fibula's head to deliver stimulation to the common } \\
\text { peroneal nerve. The foot of the leg was strapped to a } \\
\text { muscle force assessment system and was secured into a } \\
\text { boot which was fixed to a torque plate. The torque plate } \\
\text { was used to determine the optimal muscle length for } \\
\text { maximum isometric contraction by moving it until twitch } \\
\text { torques were at their maximal levels. Once the optimal } \\
\text { muscle length was determined, the isometric contraction } \\
\text { force was recorded. }\end{array}$ & $50-90 \mathrm{~V}$ & $\begin{array}{c}100 \\
\text { nanoamps }\end{array}$ & $\begin{array}{l}0.3 \\
\mathrm{~ms}\end{array}$ \\
\hline
\end{tabular}

KEYS: NR - Not Reported 
Table 8: Summary of the methodology for electrophysiological measurements

\begin{tabular}{|c|c|c|c|c|c|c|}
\hline Author(s) & \multicolumn{2}{|c|}{ Name of muscles } & Name of device & \multicolumn{2}{|c|}{ Electrodes } & Stimulation \\
\hline Llano-diez et al. (72) & NR & Tibialis anterior, Vastus lateralis & $\begin{array}{c}\text { Keypoint Medtronic, Skoulunde, } \\
\text { Denmark }\end{array}$ & $\checkmark$ & $\begin{array}{l}\text { Longitudinally over } \\
\text { the muscle fibres just } \\
\text { proximal to the distal } \\
\text { tendon insertion }\end{array}$ & $\begin{array}{c}2-5 \mathrm{KHz}, 0.1 \\
\mathrm{~ms}\end{array}$ \\
\hline Weber-Carstens et al. (105) & $\begin{array}{l}\text { Abductor pollicis } \\
\text { brevis }\end{array}$ & $\begin{array}{l}\text { Tibialis anterior, Extensor } \\
\text { digitorum brevis }\end{array}$ & $\begin{array}{c}\text { Keypoint Medtronic, Skoulunde, } \\
\text { Denmark }\end{array}$ & $\checkmark$ & NR & NR \\
\hline Weber-Carstens et al. (106) & NR & Quadriceps & STIMEX $®$ & NR & NR & NR \\
\hline
\end{tabular}

KEYS: $\checkmark$ - Yes; NR - Not Reported 


\title{
Addendum F: Ethical Approval
}

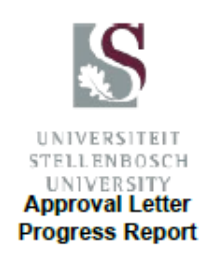

20/06/2019

Project ID: 3665

Ethics Reference No: S16/09/173A

Title: The impact of critical illness on muscle structure, function and physical capability

Dear Dr. Alison Lupton-Smith

Your request for extension/annual renewal of ethics approval dated 05/06/2019 08:05 refers.

The Health Research Ethics Committee reviewed and approved the annual progress report you submitted through an expedited review process.

The approval of this project is extended for a further year.

Approval date: 19 June 2019

Expiry date: 18 June 2020

Kindly be reminded to submit progress reports two (2) months before expiry date

Where to submit any documentation

Kindly note that the HREC uses an electronic ethics review management system, Infonetica, to manage ethics applications and ethics review process. To submit any documentation to HREC, please click on the following link: https://applyethics.sun.ac.za.

Please remember to use your Project Id 3665 and ethics reference number S16/09/173A on any documents or correspondence with the HREC concerning your research protocol.

Yours sincerely,

Ms Elvira Rohland

Health Research Ethics Committee 1 (HREC1)

\author{
National Health Research Ethics Council (NHREC) Registration Number: \\ REC-130408-012 (HREC1)•REC-230208-010 (HREC2) \\ Federal Wide Assurance Number. 00001372 \\ Office of Human Research Protections (OHRP) Institutional Review Board (IRB) Number: \\ IRB0005240 (HREC1)•IRB0005239 (HREC2)
}

The Health Research Ethics Committee (HREC) complies with the SA National Health Act No. 61 of 2003 as it pertains to health research. The HREC abides by the ethical norms and principles for research, established by the World Medical Association (2013). Declaration of Helsinki. Ethical Principles for Medical Research Involving Human
Subjects; the South African Department of Health (2006). Guidelines for Good Practice in the Conduct of Clinical Trials with Human Participants in South Africa (2nd edition); as well as the Department of Health (2015). Ethics in Health Research: Principles, Processes and Structures (2nd edition).

The Health Research Ethics Committee reviews research involving human subjects conducted or supported by the Department of Health and Human Services, or other federal departments or agencies that apply the Federal Policy for the Protection of Human Subjects to such research (United States Code of Federal Regulations Title 45 Part 46); andVor clinical investigations regulated by the Food and Drug Administration (FDA) of the Department of Health and Human Services. 


\section{Addendum G: Institutional Permission Letter}

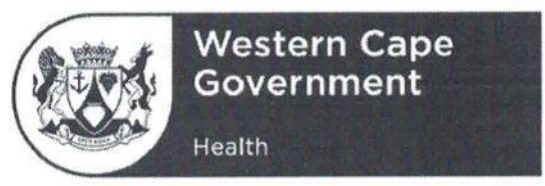

TYGERBERG HOSPITAL

REFERENCE:

Research Projects

ENQUIRIES: Dr GG

Marinus

TELEPHONE:021 9385752

Ethics Reference: S16/09/173A

TITLE: The impact of critical illness on muscle structure, strength and physical capability.

Dear Dr Alison Lupton-Smith

PERMISSION TO CONDUCT YOUR RESEARCH AT TYGERBERG HOSPITAL.

1. In accordance with the Provincial Research Policy and Tygerberg Hospital Notice No $40 / 2009$, permission is hereby granted for you to conduct the above-mentioned research here at Tygerberg Hospital.

2. Researchers, in accessing Provincial health facilities, are expressing consent to provide the Department with an electronic copy of the final feedback within six months of completion of research. This can be submitted to the Provincial Research Co-Ordinator (Health.Research@westerncape.gov.za).

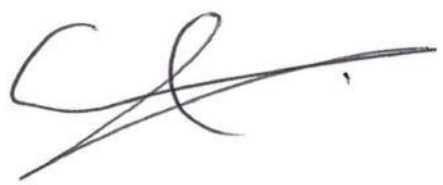

\section{DR GG MARINUS}

MANAGER: MEDICAL SERVICES

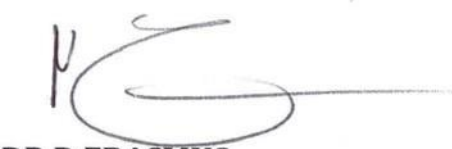

DR D ERASMUS

CHIEF EXECUTIVE OFFICER

Date: 13 Sqplambo zal8

Administration Building, Francie van Zilj Avenue, Parow, 7500

tel: $+2721938-6267$ fax: $+2721938-4890$ 
Ethics Reference: S16/09/173A

TITLE: The impact of critical illness on muscle structure, strength and physical capability.

BY

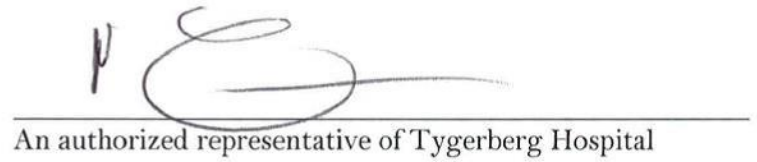

NAME Dr DS ERasmus

TITLE CEO

DATE 13 Soptanber zoi8 


\section{Addendum H: Informed Consent Letter}

\section{PARTICIPANT INFORMATION LEAFLET AND CONSENT FORM}

TITLE OF THE RESEARCH PROJECT: The impact of critical illness on muscle structure, strength and physical capability.

\section{REFERENCE NUMBER: S13/09/173A}

PRINCIPAL INVESTIGATOR: Dr Alison Lupton-Smith ADDRESS:

Stellenbosch University

Faculty of Medicine and Health Sciences

Division of Physiotherapy

Francie van Zijl Drive

Tygerberg

7505

Cape Town

South Africa

CONTACT NUMBER: 0219277191

You are being invited to take part in a research project. Please take some time to read the information which will explain the details of this project. Please ask the study staff or doctor any questions about any part of this project that you do not fully understand. It is very important that you are happy that you clearly understand what this research is about and how you could be involved. Also, your participation is entirely voluntary and you are free to say you do not want to take part. If you say no, this will not affect you negatively in any way whatsoever. You are also free to withdraw from the study at any point, even if you do agree to take part.

This study has been approved by the Health Research Ethics Committee at Stellenbosch University and will be conducted per the ethical guidelines and principles of the international Declaration of Helsinki, South African Guidelines for Good Clinical Practice and the Medical Research Council (MRC) Ethical Guidelines for Research.

\section{What is this research study all about?}

The study is being done in the intensive care units (ICU) at hospitals in South Africa. We are hoping to test most of the patients in these ICU's who had help from the breathing machine. We are hoping to test at least 400 people.

We would like to see what happens to your muscles when you have been ill in the intensive care unit (ICU). We are interested in your main breathing muscle (diaphragm) and in the muscles in your thigh (quads). We will take pictures of these muscles using ultrasound 
every day or every second day during your stay in the hospital. We will also like to see what happens to these muscles after you go home, so we will ask you to come back for tests at 3 months, 6 months and 1 year after you go home. To take these pictures we will place a probe with some gel on it onto the side of your chest and thigh. It is not painful but may feel a little cold. The pictures will be taken while you are lying in the bed. We will lift the head of the bed to $30^{\circ}$ to measure your breathing muscle. We will have to lift up your top to place the probe on your skin, but we will make sure your privacy and dignity is always respected. The pictures will be taken while you breathe in and out 3 times. To measure the thigh muscles, we will need to see the thigh, so you may have to lift you pants/skirt/dress or undress so that we can see your thigh. These measurements will also take place while you are lying down.

We will also ask you to perform some tests to see how strong your muscles are and to see what you are and are not able to do. To test the strength of your breathing muscles we will ask you to breathe in and out as hard as you can through a machine while sitting. To test the strength of your arms and legs we will ask to push against us or a machine and do certain movements. To test what you can and can't do we will ask you to do certain tasks like rolling in the bed, sitting, standing, and walking for six minutes. If at any time during these tests you feel tired, you should let the investigator know and you will be given a chance to rest. None of the tests we are doing are new, all of them have been used in South Africa and around the world before.

We would also like to find out if being in the ICU had any effect on your mental health. We will be looking for things such as depression, anxiety, post-traumatic stress disorder and your ability to perform simple tasks. To find this out we will ask you some questions about your time in the ICU and how you are feeling. We will also ask you to perform a few tasks. We will collect information about why you came to the hospital and what tests were done while you were in hospital. We will ask about what other conditions you have and what you were doing before you came to hospital (like were you working or retired). We will also ask you some questions about how you feel about your health and if you have had to visit doctors and hospitals after you are discharged from the hospital. We would also like to know how much being in the ICU cost you. To do this, we will ask you questions about your work, how much money you are paid and if you have any government grants.

We would also like to find out if and how the food you get, and vitamin D levels, affects your muscles and how easily you can do things like moving in the bed, walking, washing and dressing. We will take measurements like your height and weight and record the type of 
food/nutrition you receive while in the ICU. We will also record any things (like dialysis) which can affect how much food/nutrients you get, we will get this information from your hospital folder. To measure the vitamin D levels, we will need to draw a small amount (teaspoon $-5 \mathrm{ml}$ ) of blood in the first 24 hours you are in the intensive care unit. Your name and details will not be on the blood sample, so no one working with the blood will know that it is yours. The samples will be thrown away once they have been tested.

\section{Why have you been invited to participate?}

We have asked you to take part in this study because you are/were getting help from the machine to breath and are/were in one the two ICU's at one of the hospitals we are doing the study in.

\section{What will your responsibilities be?}

What we would need from you, is your co-operation with the tests. The tests should take about 2-3 hours, but this may vary. We would also like to know that you will be willing to return for follow-up visits at 3,6 and 12 months after you are discharged from hospital. If at any time during the studies you decide you no longer want to take part, that is fine and it will not affect your medical care in any way.

\section{Will you benefit from taking part in this research?}

There is no direct benefit to you from taking part in the study, but the results from this study may help improve the care of people in the ICU. Should we find anything that we feel your Doctor should know about during our tests, we will inform your Doctor with your permission.

\section{Are there in risks involved in your taking part in this research?}

There are no risks in taking part in this study. None the tests are painful or have any side effects. You may feel tired during the tests, if this is the case you will be allowed to rest until you are ready to continue. If you have any conditions that prevent you from being tested, we will not perform the tests.

\section{If you do not agree to take part, what alternatives do you have?}

Nothing will happen if you don't want to take part in the study. Your choice not to take part will not affect your medical care in any way.

\section{Who will have access to your medical records?}

Only the investigators taking the measurements will have access to your medical records. All information will be stored anonymously (people won't know the information belongs to 
you). The information will be stored on password protected laptop and database. Participants will remain anonymous in any publications or research outputs.

What will happen in the unlikely event of some form injury occurring as a direct result of your taking part in this research study?

In the rare event that you sustain an injury during the study tests, your doctor and medical team will be notified immediately. As a participant you are also covered by insurance from the University of Stellenbosch.

Will you be paid to take part in this study and are there any costs involved?

No, you will not be paid to take part in the study, but your transport and meal costs will be covered for each study visit out of the hospital. There will be no costs involved for you, if you do take part.

Is there anything else that you should know or do?

You can contact Dr Alison Lupton-Smith at tel 0219277191 if you have any further queries or encounter any problems.

You can contact the Health Research Ethics Committee at 021-938 9207 if you have any concerns or complaints that have not been adequately addressed by your study doctor.

You will receive a copy of this information and consent form for your own records.

\section{Can we contact you for future research?}

You will be involved in this research for one year after your discharge from hospital. In the future we may want to contact you after a year for further research to find out how you are doing.

You may contact me for future studies $\quad \square$ Yes $\quad \square$ No

\section{Declaration by Participant}

By signing below, I agree to take part in a research

study entitled the impact of critical illness on muscle structure, strength and physical capability.

I declare that:

- I have read or had read to me this information and consent form and it is written in a language with which I am fluent and comfortable. 
- I have had a chance to ask questions and all my questions have been adequately answered.

- I understand that taking part in this study is voluntary and I have not been pressurised to take part.

- I may choose to leave the study at any time and will not be penalised or prejudiced in any way.

- I may be asked to leave the study before it has finished, if the study doctor or researcher feels it is in my best interests, or if I do not follow the study plan, as agreed to.

\section{Signature of Participant}

\section{Declaration by Investigator}

I (name)

- I explained the information in this document to

- I encouraged him/her to ask questions and took adequate time to answer them.

- I am satisfied that he/she adequately understands all aspects of the research, as discussed above

- I did/did not use an interpreter. (If an interpreter is used then the interpreter must sign the declaration below.

Signed at (place) on (date) 20

\section{Signature of Investigator}

\section{Signature of Witness}

\section{Declaration by Interpreter}

- I (name) declare that:

- I assisted the investigator (name) to explain the information in this document to (name of participant) using the language medium of Afrikaans/Xhosa.

- We encouraged him/her to ask questions and took adequate time to answer them.

- I conveyed a factually correct version of what was related to me. 
- I am satisfied that the participant fully understands the content of this informed consent document and has had all his/her question satisfactorily answered.

Signed at (place) on (date)

20 
Research ID:

\section{DEELNEMERINLIGTINGSBLAD EN -TOESTEMMINGSVORM}

TITEL VAN DIE NAVORSINGSPROJEK: Die impak van kritieke siekte op spiersamestelling, -sterkte en fisiese vermoëns.

VERWYSINGSNOMMER: S13/09/173A

HOOFNAVORSER: Dr Alison Lupton-Smith

ADRES:

Universiteit Stellenbosch

Fakulteit Geneeskunde en Gesondheidswetenskappe

Afdeling van Fisioterapie

Francie van Zijl laan

Tygerberg

7505

Kaapstad

Suid-Afrika

KONTAKNOMMER: 0219277191

$\mathrm{U}$ word uitgenooi om deel te neem aan 'n navorsingsprojek. Lees asseblief hierdie inligtingsblad op u tyd deur aangesien die detail van die navorsingsprojek daarin verduidelik word. Indien daar enige deel van die navorsingsprojek is wat u nie ten volle verstaan nie, is u welkom om die navorsingspersoneel of dokter daaroor uit te vra. Dit is baie belangrik dat $u$ ten volle moet verstaan wat die navorsingsprojek behels en hoe u daarby betrokke kan wees. U deelname is ook volkome vrywillig en dit staan u vry om deelname te weier. $U$ sal op geen wyse hoegenaamd negatief beïnvloed word indien u sou weier om deel te neem nie. U mag ook ter enige tyd aan die navorsingsprojek onttrek, selfs al het $u$ ingestem om deel te neem.

Hierdie navorsingsprojek is deur die Gesondheidsnavorsingsetiekkomitee (GNEK) van die Universiteit Stellenbosch goedgekeur en sal uitgevoer word volgens die etiese riglyne en beginsels van die Internasionale Verklaring van Helsinki en die Etiese Riglyne vir Navorsing van die Mediese Navorsingsraad (MNR). 


\section{Research ID:}

\section{Wat behels hierdie navorsingsprojek?}

Hierdie studie word uitgevoer in die intensiewe sorg eenhede van verskeie hospitale in SuidAfrika. Ons hoop om alle pasiënte wat met behulp van ' $n$ asemhalingsmasjien asemhaal, te toets. Ons hoop om minstens 400 pasiënte te toets.

Ons wil graag vasstel hoe u verblyf in die intensiewe sorgeenheid (ISE) u spiere affekteer. Ons is veral geinteresseerd in u hoof asemhalingsspier, die diafragma, asook in u bobeenspier (quads). Ons sal daagliks of elke tweede dag tydens u hospitaalverblyf fotos van hierdie spiere neem met behulp van ' $n$ ultraklankmasjien. Verder wil ons ook graag hierdie spiere toets na u ontslag uit die hospitaal, dus sal ons u vra om terug te kom vir soortgelyke toetse 3-, 6-, en 12 maande na u hospitaalontslag. Om hierdie fotos te neem, sal ons ' $n$ probe met jellie op u borskas en bobeen plaas. Dit sal nie seer wees nie, maar mag dalk bietjie ongemaklik voel. Die fotos sal geneem word terwyl u in u hospitaalbed lê. Ons sal die kopstuk van u hospitaalbed tot 30 grade lig wanneer ons fotos van jou asemhalingsspier neem. Ons sal u hemp moet oplig om sodoende die probe op u borskas te plaas, maar sal ten alle tye u privaatheid en menslikheid respekteer. Die fotos sal geneem word terwyl u drie keer in- en uitasem. Om u bobeenspier te meet, sal ons u vra om u broek/romp/rok op te lig sodat ons u bobeen kan sien. Hierdie metings sal ook geneem word terwyl u in die bed lê.

Ons sal u ook versoek om sekere toetse uit te voer om te sien hoe sterk u spiere is en om te sien tot wat $u$ in staat is om te doen. Om die krag van $u$ asemhalingspier te toets, sal ons $u$ vra om so hard as moontlik deur ' $n$ masjien in- en uit te asem. Om die krag van u arms en bene te toets, sal ons u vra om teen ons of 'n masjien te druk en sekere bewegings uit te voer. Om te toets wat u kan en nie kan doen nie, sal ons u vra om sekere take uit te voer, soos om in die bed te rol, sit, staan en te loop vir ses minute. As u op enige tydstip moeg voel, moet u die navorser daarvan laat weet en die kans kry om te rus.

Nie een van die toetse wat ons doen, is nuut nie, hulle is al voorheen in Suid-Afrika en wêreldwyd uitgevoer.

Ons wil graag ook uitvind of u verblyf in die ISE u geestesgesondheid beïnvloed het. Ons sal ondersoek instel na faktore soos depressie, angs, post-traumatiese stresversteuring en u vermoë om eenvoudige take uit te voer. Om dit uit te vind, sal ons u vrae vra oor u verblyf in die ISE en hoe u voel. Ons sal u ook versoek om enkele take uit te voer.

Ons sal inligting insamel oor hoekom u in die hospitaal opgeneem is, asook watter toetse tydens u hospitaalverblyf op u uitgevoer is. Ons sal u ook vra of $u$ aan enige ander toestande lei, asook wat $u$ gedoen het voordat $u$ in die hospitaal opgeneem is (soos of u gewerk het of afgetree is). Ons sal u ook vrae vra oor hoe u oor u gesondheid voel, en of u dokters en hospitale moes besoek nadat u uit die hospitaal ontslaan is. Ons wil ook graag ook uitvind 


\section{Research ID:}

hoeveel die versorging in die ISE u gekos het. Om dit te doen, sal ons u vrae vra oor u werk, salaris en/of $u$ enige regeringstoelaes ontvang.

Verder wil ons graag uitvind hoe u voedselinname tydens u ISE verblyf, asook u bloed vitamien D-vlakke, u spiere beïnvloed het en hoe maklik u sekere aktiwiteite kan uitvoer soos om in die bed te beweeg, te loop, te was en aan te trek. Ons sal metings soos u lengte en gewig neem, asook vasstel watter tipe en hoeveelheid voedsel / voeding wat u tydens u ISE verblyf ontvang het. Ons sal ook enige faktore (soos dialise) aanteken wat die hoeveelheid voedsel / voedingstowwe wat u kry, kan beïnvloed. Ons sal hierdie inligting uit u hospitaalleër kry. Om die vitamien D-vlakke te meet, moet ons ' $n$ klein hoeveelheid (teelepel - $5 \mathrm{ml}$ ) bloed trek in die eerste 24 uur wat $u$ in die ISE is. $U$ naam en besonderhede is nie op die bloedmonster nie, en niemand wat met die bloed werk, sal weet dat dit u s'n is nie. Die monsters sal weggegooi word sodra dit getoets is.

\section{Waarom is u uitgenooi om deel te neem?}

Ons het u gevra om aan hierdie studie deel te neem, omdat u toegelaat is/was in een van die ISE wat ingesluit is in hierdie studie, asook met behulp van ' $n$ asemhalingsmasjien asemhaal,

\section{Wat sal u verantwoordelikhede wees?}

Wat van u benodig word, is u samewerking met die toetse. Die toetse behoort ongeveer 2-3 uur te neem, maar dit kan wissel. Ons wil ook weet of u bereid sal wees om terug te keer vir opvolgbesoeke op 3, 6 en 12 maande nadat $u$ uit die hospitaal ontslaan is. $U$ kan op enige tydstip tydens die studie besluit dat u nie meer wil deelneem nie, en dit sal geensins $u$ mediese sorg op geen enkele manier beïnvloed nie.

Sal u daarby baat vind om aan hierdie navorsing deel te neem? Of sal jy voordeel trek deur deel te neem aan hierdie navorsingsprojek?

Daar is geen direkte voordeel vir $\mathrm{u}$ om aan die studie deel te neem nie, maar die resultate van hierdie studie kan die versorging van mense in die ISU help verbeter. Indien ons iets vind tydens die toetse waarvan u dokter moet weet, sal ons u dokter daarvan inlig, mits $u$ toestemming daarvoor gee.

\section{Is daar enige risiko's verbonde aan u deelname aan hierdie navorsingsprojek?}

Daar is geen risiko's verbonde aan u deelname aan hierdie navorsingsprojek nie. Nie een van die toetse is pynlik of het newe-effekte nie. $U$ mag wel moeg voel tydens die toetse, as dit die geval is, sal u toegelaat word om te rus totdat u gereed is om voort te gaan. Sommige pasiënte mag ook effense ongemak ervaar gedurende die neem van die bloedmonster. As u enige voorwaardes het wat u verhinder om getoets te word, sal ons nie die toetse uitvoer nie. 
Research ID:

As $u$ nie instem om deel te neem nie, watter alternatiewe het u?

Daar sal geen implikasies wees indien u besluit om nie aan die studie deel te neem nie. $U$ keuse om nie deel te neem nie, sal u mediese sorg op geen enkele manier beïnvloed nie.

Wie het toegang tot u mediese rekords?

Slegs die navorsers wat die metings doen, het toegang tot u mediese rekords. Alle inligting sal anoniem geberg word (mense weet nie dat die inligting aan u behoort nie). Die inligting word op 'n skootrekenaar en databasis beskerm deur 'n wagwoord. Deelnemers sal anoniem bly in enige publikasie of navorsingsuitsette.

Wat sal gebeur in die onwaarskynlike geval dat daar een of ander vorm van besering sal plaasvind as gevolg van u deelname aan hierdie navorsingstudie?

In die seldsame geval dat u tydens die toetse 'n besering opdoen, sal u dokter en mediese span onmiddellik in kennis gestel word. As deelnemer word u ook verseker deur die Universiteit van Stellenbosch.

Sal u betaal word om aan hierdie studie deel te neem en is daar kostes daaraan verbonde?

Nee, u sal nie betaal word om aan die studie deel te neem nie, maar u vervoer- en maaltydkoste word gedek vir elke studiebesoek buite die hospitaal. U sal geen koste daaraan verbonde hê as u wel deelneem nie.

Is daar iets anders wat u moet weet of doen?

U kan Dr Alison Lupton-Smith kontak by tel 0219277191 indien u enige verdere vrae het of probleme ondervind.

U kan die Kommitee vir Gesondheidsnavorsingsetiek by 021-9389207 kontak indien u enige kommer of klagtes het wat nie voldoende deur u studielokaal aangespreek is nie.

$\mathrm{U}$ sal ' $\mathrm{n}$ afskrif van hierdie inligting en toestemmingsvorm vir u eie rekords ontvang. 


\section{Research ID:}

\section{Kan ons u kontak vir toekomstige navorsing?}

$\mathrm{U}$ sal vir een jaar na u ontslag uit die hospitaal by hierdie navorsing betrokke wees. In die toekoms wil ons u miskien na 'n jaar kontak vir verdere navorsing om uit te vind hoe $u$ te werk gaan.

U kan my kontak vir toekomstige studies $\quad \square \mathrm{Ja} \quad \square$ Nee

\section{Verklaring deur deelnemer}

Met die ondertekening van hierdie dokument onderneem ek,

om deel te neem aan 'n navorsingsprojek getiteld: Die impak van kritieke siekte op spiersamestelling, -sterkte en fisiese vermoëns.

- Ek verklaar dat:

- Ek hierdie inligtings - en toestemmingsvorm gelees het of aan my laat voorlees het en dat dit in ' $n$ taal geskryf is waarin ek vaardig en gemaklik mee is.

- Ek geleentheid gehad het om vrae te stel en dat al my vrae bevredigend beantwoord is.

- Ek verstaan dat deelname aan hierdie studie vrywillig is en dat daar geen druk op my geplaas is om deel te neem nie.

- Ek te eniger tyd aan die navorsingsprojek mag onttrek en dat ek nie op enige wyse daardeur benadeel sal word nie.

- Ek gevra kan word om die studie te verlaat voordat dit voltooi is, as die studielid of navorser van mening is dat dit in my beste belang is, of as ek nie die studieplan, soos ooreengekom, volg nie.

\section{Verklaring deur navorser:}

Ek (naam) verklaar dat: verduidelik. 


\section{Research ID:}

- Ek het hom / haar aangemoedig om vrae te stel en het voldoende tyd geneem om dit te beantwoord.

- Ek is tevrede dat hy / sy, soos hierbo bespreek, die aspekte van die navorsing voldoende verstaan

- Ek het / het nie 'n tolk gebruik nie. (As 'n tolk gebruik word, moet die tolk die verklaring hieronder onderteken.

Geteken te (plek)

op (datum) $20 \ldots \ldots$

Handtekening van navorser

\section{Handtekening van getuie}

\section{Verklaring deur tolk:}

Ek (naam) verklaar dat:

- Ek het die navorser (naam) bygestaan om die inligting in hierdie dokument aan (naam van die deelnemer) te verduidelik. met behulp van die taalmedium Afrikaans / Xhosa.

- Ons het hom / haar aangemoedig om vrae te stel en het voldoende tyd geneem om dit te beantwoord.

- Ek het 'n feitelik korrekte weergawe oorgedra van wat met my verband hou.

- Ek is tevrede dat die deelnemer die inhoud van hierdie ingeligte toestemmingsdokument ten volle begryp en dat al sy / haar vrae bevredigend beantwoord is. 
Research ID:

INCWADANA YEENKCUKACHA YALOWO UTHABATHA INXAXHEBA NEFOMU YOKUNIKA IMVUME

\begin{tabular}{|c|c|}
\hline \multicolumn{2}{|l|}{ ISIHLOKO SEPROJEKTHI YOPHANDO: } \\
\hline \multicolumn{2}{|c|}{$\begin{array}{l}\text { Impembelelo yesifo esibuhlungu kwisakhiwo se-muscle, amandla kunye nokukwazi ukwenza } \\
\text { umzimba }\end{array}$} \\
\hline \multicolumn{2}{|l|}{ IINKCUKACHA ZOMPHANDI OYINTLOKO (PI): } \\
\hline Isihlonipho, igama lokuqala, ifani: Dr Alison Lupton-Smith & $\begin{array}{l}\text { Inombolo yesingqinisiso } \\
\text { kwiNdlela yokuziphatha: } \\
\text { S16/09/173A }\end{array}$ \\
\hline $\begin{array}{l}\text { Idilesi epheleleyo yeposi: KwiYunivesithi yeStellenbosch } \\
\text { I-Faculty of Medicine neSayensi yezeMpilo } \\
\text { Ulwahlulo IwePhysiotherapy } \\
\text { UFrancie van Zijl Drive } \\
\text { ITygerberg } \\
7505 \\
\text { Kapa } \\
\text { Mzantsi Afrika }\end{array}$ & $\begin{array}{l}\text { Inombolo epheleleyo } \\
\text { yoMphandi oyiNtloko: } \\
021-9277191\end{array}$ \\
\hline
\end{tabular}

Uyamenywa ukuba uthathe inxaxheba kwiprojekthi yophando. Nceda uthathe ixesha lokufunda ulwazi oluya kuchaza iinkcukacha zeprojekthi. Nceda ucele abasebenzi abafundayo okanye ugqirha nayiphi na imibuzo malunga nayiphi na inxalenye yale projekthi ongaziqondi ngokupheleleyo. Kubaluleke kakhulu ukuba uyavuya ukuba uyayiqonda ngokucacileyo ukuba lo uphando uphathelene nani nokuthi ungabandakanyeka njani. Kwakhona, ukuthatha inxaxheba ngokuzithandela ngokupheleleyo kwaye ukhululekile ukuthetha ukuba awufuni ukuthatha inxaxheba. Ukuba uthe hayi, oku akukuchaphazela kakubi nangayiphi na indlela. Ukhululekile ukuba uhoxise kwisifundo nakweyiphi na indawo, nokuba uyavuma ukuthatha inxaxheba.

Olu phofu luvunyiwe yiKomidi yoLwazi IwezeMpilo kwiYunivesithi yaseStellenbosch kwaye iya kuqhutywa ngeemeko zomgaqo-nkqubo kunye neMigqaliselo ye-Helsinki yamazwe ngamazwe, Izikhokelo zaseMzantsi Afrika zeCandelo leMpilo yeCliniki kunye neBhunga loPhando IwezeMpilo (MRC) Izikhokelo zoPhando zoLwazi. 
Research ID:

\section{Lumalunga nantoni olu phando?}

Isifundo senziwa kwiiyunithi zokunyamekela kakhulu (ICU) kwizibhedlele eMzantsi Afrika. Sithemba ukuvavanya amaninzi kwezigulane kula ma-ICU awancedayo kumshini wokuphefumula. Sithemba ukuvavanya ubuncinane abantu abangama-400

Sithanda ukubona ukuba kwenzekani kwiimisipha zakho xa ugula kwiyunithi yonyango enzulu (ICU). Sinesithakazelo kwisisipha sakho sokuphefumla (i-diaphragm) kunye nakumisipha entanyeni yakho (quads). Siza kuthatha imifanekiso yale misipha usebenzisa i-ultrasound imihla ngemihla okanye yonke imihla yesibini ngexesha lokuhlala esibhedlele. Siyakuthanda ukubona ukuba kwenzeke ntoni kwezi zihlunu emva kokuba uhambe ekhaya, ngoko siya kukucela ukuba ubuyele kwiimvavanyo kwiinyanga ezintathu, ezintandathu neenyanga 1 emva kokuba uye ekhaya. Ukuthatha le mifanekiso siya kufaka iprojekti kunye nejel kuyo kwicala lesifuba sakho nentambo. Akubuhlungu kodwa kunokuziva kubanda kakhulu. Imifanekiso iya kuthathwa xa ulala embhedeni. Siza kuphakamisa intloko yebhedi ukuya kwi-30 ${ }^{\circ}$ ukulinganisa umsizi wakho wokuphefumula. Siya kuphakanyiswa phezulu sakho ukubeka iprojekti esikhumbeni sakho, kodwa siya kuqinisekisa ukuba ubucala bakho kunye nesidima sihlala sihlonishwa. Imifanekiso iya kuthathwa xa uphefumula kwaye uphuma maxesha amathathu. Ukulinganisa imisipha yamathanga, kuya kufuneka ukuba sibone intamo, ngoko kufuneka uphakamise incindi / iingubo / ugqoke okanye ugxininise ukuze sikwazi ukubona intamo yakho. Le milinganiselo iya kwenzeka kwakhona xa ulala phantsi.

Siyakukucela ukuba wenze iimvavanyo ezithile ukuze ubone indlela ezinamandla ngayo iimisipha zakho kwaye ubone ukuba ungubani kwaye awukwazi ukwenza. Ukuvavanya amandla okuphefumula kwakho siyakukucela ukuba uphefumle kwaye uphumelele njengokuba unako ngomshini ngelixa uhleli. Ukuvavanya amandla eengalo kunye nemilenze yakho esiya kucela ukuba sisitshintshe kuthi okanye umatshini kwaye senze uhambo oluthile. Ukuvavanya into onokuyenza kwaye akunako ukwenza siza kukucela ukuba wenze imisebenzi ethile efana nokugqitha ebhedini, uhleli, umi, kwaye uhamba imizuzu emithandathu. Ukuba naliphi na ixesha kule mivavanyo uziva ulele, kufuneka uvumele umphenyi azi kwaye uya kunikwa ithuba lokuphumla.

Akukho mvavanyo esenzayo yintsha, zonke ziye zasetyenziswa eMzantsi Afrika nakwihlabathi eliphambili.

Sifuna kwakhona ukufumanisa ukuba ukuba i-ICU inayo nayiphi na impembelelo kwimpilo yakho yengqondo. Siza kukhangela izinto ezifana nokudandatheka, ukuxhalabisa, ukuphazamiseka kwengxaki yokuhamba ngengxaki kunye nokukwazi ukwenza imisebenzi 
Research ID:

elula. Ukukufumanisa oku siza kukubuza imibuzo malunga nexesha lakho kwi-ICU nendlela ovakalelwa ngayo. Siyakukucela ukuba wenze imisebenzi embalwa.

Siza kuqokelela ulwazi malunga nokuba ufike esibhedlele kwaye ziphi iimvavanyo zenziwa ngelixa usesikhungweni. Siza kubuza malunga naziphi ezinye iimeko kunye nokuba wenza ntoni ngaphambi kokuba ufike esibhedlele (njengokuba wawusebenza okanye uthatha umhlalaphantsi). Siza kucela kwakhon unemibuzo malunga nendlela ozivakalelwa ngayo ngempilo yakho kwaye ukuba ufanele ukutyelela oogqirha nezibhedlele emva kokuba ukhishwe esibhedlele. Sithanda kwakhona ukwazi ukuba kukhulu kangakanani ukuhlala kwi-ICU. Ukwenza oku, siza kukubuza imibuzo malunga nomsebenzi wakho, imali ehlawulwa ngayo kwaye ukuba unayo nayiphi na inkxaso-karhulumente.

Sifuna kwakhona ukufumana ukuba kunye nokutya okufumanayo, kunye namanqanaba evitamin D, ichaphazela iimisipha zakho kwaye kulula kangakanani ukwenza izinto ezifana nokuhamba esiteni, ukuhamba, ukuhlamba nokugqoka. Siza kuthatha amanani afana nokuphakama nobukhulu bakho kwaye urekhode uhlobo lokutya / ukondla ofumanayo ngexesha le-ICU. Siza kubhala kwakhona izinto (ezifana ne-dialysis) ezinokuchaphazela ukuba ufumana ukutya / izondlo ezininzi, siya kufumana le ngcaciso kwifolda yakho yezibhedlele. Ukulinganisa amazinga e-vitamin $\mathrm{D}$, kuya kufuneka sibe nomlinganiselo omncinci (i-teaspoon $5 \mathrm{ml}$ ) yegazi kwiiyure ezingama-24 zokuqala unguncinci yokukhathalela. Igama lakho kunye neenkcukacha aziyi kuba sesampula segazi, ngoko akukho mntu osebenza ngegazi uya kukwazi ukuba ngowakho. lisampula ziya kuphoswa xa zihlolwe.

\section{Kutheni uceliwe ukuba uthathe inxaxheba?}

Sikucelile ukuba uthathe inxaxheba kulolu cwaningo ngenxa yokuba ufumana / uncedo oluvela kumatshini ukuphefumla kwaye / bekho kwenye yee-ICU ezimbini kwenye yezibhedlele esizenzayo.

\section{Luya kuba yintoni uxanduva Iwakho?}

Into esiyidingayo kuwe, kukusebenza kwakho kunye neemvavanyo. Imvavanyo kufuneka ithathe malunga neeyure eziyi-2-3, kodwa oku kuyahluka. Sifuna kwakhona ukwazi ukuba uya kukulungela ukubuyela ekuhambelweni okulandelelweyo kwiinyanga ezi-3, 6 neye-12 emva kokuba ukhupha esibhedlele. Ukuba nayiphi na ixesha ngexesha lophando uthatha isigqibo sokuba awusayi kufuna ukuthatha inxaxheba, oku kulungile kwaye akuyi kuphazamisa ukunakekelwa kwakho kwonyango nganoma iyiphi indlela 
Research ID:

Ingaba uya kuzuza ngokuthatha kwakho inxaxheba kolu phando?

Akukho nzuzo ngokuthe ngqo kuwe ekubeni uthathe inxaxheba kwisifundo, kodwa iziphumo ezivela kule sifundo zinokunceda ukuphucula ukunakekelwa kwabantu kwi-ICU.

Ngaba sifanele sifumene nantoni na esiyicinga ukuba ugqirha kufuneka uyazi malunga neemvavanyo zethu, siya kuzisa ugqirha wakho ngemvume yakho

Ingaba kukho imingcipheko ebandakanyekayo ekuthatheni kwakho inxaxheba kolu phando?

Akukho mingcipheko ekuthatheni inxaxheba kule sifundo. Azikho iimvavanyo ezibuhlungu okanye zinemiphumo emibi. Unokuziva unxinekile ngexesha leemvavanyo, ukuba ngaba kunjalo uya kuvunyelwa ukuphumla uze ulungele ukuqhubeka. Ukuba unemiqathango ekhusela ukuba uvavanywe, asiyi kuyenza iimvavanyo.

Ukuba akuvumi ukuthatha inxaxheba, zeziphi ezinye iindlela onazo ezinokulandelwa? Akukho nto iya kwenzeka xa ungafuni ukuthatha inxaxheba kwisifundo. Ukukhetha kwakho ukuba ungathathi inxaxheba akusayi kuchaphazela unyango Iwakho ngonyango

\section{Ngubani oza kukwazi ukufikelela kwiingxelo zakho zezonyango?}

Kuphela abaphandi abenza imilinganiselo baya kuba nokufikelela kwiirekhodi zakho zonyango. Yonke ingcaciso iya kugcinwa ngokungachazwanga (abantu abayikwazi ukuba ulwazi luniwe). Ingcaciso iya kugcinwa kwi-laptop ekhuselweyo i-laptop kunye nedatha. Abathathi-nxaxheba baya kuhlala bengaziwa naziphi na iimpapasho okanye iziphumo zophando

Noxa kungaqhelanga kwenzeka, kuza kwenzeka ntoni xa unokwenzakala nangayiphi indlela kuba uthatha inxaxheba kolu phando?

Kwimeko enqabileyo yokuba ugcina ukulimala ngexesha lovavanyo Iweemvavanyo, ugqirha lakho kunye neqela lezonyango liya kwaziswa ngokukhawuleza. Njengothathi-nxaxheba ufunyenwe ne-inshorensi evela kwiYunivesithi yeStellenbosch

Ingaba uza kuhlawulwa ngokuthatha inxaxheba kolu phando kwaye ingaba kukho indleko ezibandakanyekayo?

Hayi, awuyi kuhlawulwa ukuba uthathe inxaxheba kwisifundo kodwa iindleko zakho zokuthutha kunye nezokutya ziya kugutyungelwa ukutyelela nganye kwisibhedlele. Ngeke kubekho iindleko ezichaphazelekayo kuwe, ukuba uthatha inxaxheba. 
Research ID:

Ingaba ikhona enye into ekufuneka ukuba uyazi okanye uyenze?

- Unokuqhagamshelana noDkt. Alison Lupton-Smith kwifowuni 0219277191 ukuba unemibuzo engaphezulu okanye uhlangabezana nazo naziphi na iingxaki.

- Unokuqhagamshelana neKomidi yoLwazi IwezeMpilo kwi-021-938 9207 ukuba unenkxalabo okanye izikhalazo ezingakhange ziqwalaselwe ngokwaneleyo ngudokotela wakho wokufunda.

- Uya kufumana ikopi yale ngcaciso kunye nefom yemvume kwiiirekhodi zakho 


\section{Research ID:}

Ngaba sinokuqhagamshelana nawe ngophando olusasa?

Uya kubandakanyeka kulolu cwaningo unyaka owodwa emva kokuphuma kwakho esibhedlele. Kwixesha elizayo sinokufuna ukunxibelelana nawe emva konyaka ukuze uphando olungakumbi ukuze ubone ukuba wenza ntoni

Unokuqhagamshelana nam ngeenjongo ezizayo $\square$ Ewe $\square$ Hayi

\section{Isifungo somthathi-nxaxheba}

Ngokusayinela ngezantsi, mna uyavuma ukuthatha inxaxheba kwiphando lophando olubhaliweyo elinomxholo othi: Impembelelo yesifo esibuhlungu kwisakhiwo se-muscle, amandla kunye nokukwazi ukwenza umzimba

\section{Ndiyaxela ukuba:}

Ndiyifunde okanye ndifundele le ngcaciso kunye nefomu yemvume kwaye ibhaliwe ngolwimi endilungeleyo kwaye lukhululekile.

- Ndiye ndafumana ithuba lokubuza imibuzo kwaye yonke imibuzo yam iphendulwe ngokufanelekileyo

- Ndiyaqonda ukuba ukuthatha inxaxheba kule sifundo kukuzithandela kwaye andizange ndixinzezeleke ukuba ndithathe inxaxheba.

- Ndinokukhetha ukushiya isifundo nganoma naliphi ixesha kwaye andiyi kuhlawulwa okanye ngandlela-thile naluphi na uhlobo.

- Ndinokucelwa ukuba ndishiye isifundo ngaphambi kokugqiba, ukuba ugqirha wophando okanye umphandi unomuva onyanisekileyo, okanye ukuba andilandelanga isicwangciso sokufunda, njengoko kuvunyelwene

Kutyikityelwe (indawo) engomhla (umhla) we-

ngo-20... 
Research ID:

Isibhengezo somphandi

Mna (igama) ndibhengeza ukuba:

- Ndizicacisile iinkcukacha ezikolu xwebhu ngendlela elula necacileyo ku.

- Ndimkhuthazile ukuba abuze imibuzo ndaza ndathatha ixesha elaneleyo ukuyiphendula.

- Ndanelisekile kukuba uyiqonda ngokwaneleyo yonke imiba yolu phando, njengoko icacisiwe apha ngentla.

Kutyikityelwe (indawo) ngomhla (umhla) we-

ngo-20..

Utyikityo lomphandi

Utyikityo Iwengqina

Isibhengezo ngokutolika

Ndiza (igama) ngithi:

- Ndikuncede umphandi (igama) ukuchazela ulwazi olu xwebhu (igama lomntu othabatha inxaxheba) usebenzisa ulwimi lolwimi IwesiBhulu / isiXhosa.

- Siyamkhuthaza ukuba abuze imibuzo kwaye athathe ixesha elaneleyo lokuphendula.

- Ndahambisa ingcaciso echanekileyo yento edibeneyo kum.

- Ndiyanelisekile ukuba umntu ochaphazelekayo uyaziqonda ngokupheleleyo umxholo wale ngxelo yemvume eneenkcukacha kwaye umbuzo wakhe uphendule ngokufanelekileyo.

Isayinwe kwindawo (indawo) ngomhla (umhla)

Isayinithi yetoliki IsiTyikityo 


\section{Addendum I: Pilot Study}

\section{Pilot Study}

This pilot study was conducted before the commencement of the primary study.

\section{Objectives:}

To determine the intra-rater and inter-rater reliability of the ultrasound measurements of the diaphragm and quadriceps muscle.

\section{Methods:}

Setting: This pilot study was conducted at Tygerberg Academic Hospital, in the Surgical (A1) Intensive Care Unit (ICU).

Ethical considerations: All participants provided informed consent.

Sample: Four intubated and mechanically ventilated participants were included for the ultrasound measurements.

\section{Measurements:}

- Procedure of ultrasonography of the diaphragm and quadriceps muscle

Four intubated and mechanically ventilated participants were screened in the Surgical (A1) ICU of Tygerberg Academic Hospital and were included in this pilot study. Study information and procedure were explained to the participants, and informed consent was obtained. Ultrasonography of the diaphragm and quadriceps muscle was performed using a Phillips Lumify portable ultrasound machine in B-mode, using a 7-12 $\mathrm{MHz}$ linear probe. The right hemi-diaphragm muscle was imaged at the zone of apposition and the probe was placed against the chest wall, on the right anterior axillary or mid-axillary line between the eighth and tenth intercostal space depending on where the clearest image appeared. A watersoluble gel was applied to the ultrasound linear probe to allow for acoustic contact without depressing the dermal surface. Participants were positioned in a $30^{\circ}$ semi-recumbent position. Images of the diaphragm were recorded in loops of three full tidal volume breathing cycles, from inspiration to expiration. The co-investigator (SC) first recorded three consecutive breaths, and thereafter the primary investigator (PI) (ALS) recorded three consecutive breaths on the same participant.

The right thigh was used for measurements of the Rectus Femoris (RF) and Vastus Intermedius ( $\mathrm{VI}$ ) muscle thickness and echogenicity, on anterior imaging. The ultrasound probe was placed perpendicular to the long axis of the anterior thigh, two-thirds from the anterior superior iliac spine (ASIS) to the superior patella border. The co-investigator first recorded two images of the RF and VI muscles, and thereafter the $\mathrm{PI}$ recorded two images of the RF and VI muscles on the same participant. 
- Intra-rater reliability:

The PI and the co-investigator repeated the procedure twice on each participant, with a 30minute interval between the first and second measurements in order to establish intra-rater reliability of the separate ultrasound measurements. In both measurements the exact positioning, ultrasonography equipment and setting was used. Results are shown in Table 1.

- Inter-rater reliability:

The diaphragm muscle thickness, quadriceps muscle thickness and echogenicity measurements of the four participants taken by the PI was compared to the co-investigator's measurements in order to establish inter-rater reliability. This was done to establish ultrasound measurements between the raters and to evaluate the co-investigator's ultrasound training. The exact same positioning, ultrasonography equipment and setting was used. Results are shown in Table II.

\section{Data analysis}

Intraclass correlation coefficients (ICC) were used in order to establish intra- and interrater reliability. Absolute agreement with a $95 \%$ confidence interval was selected. An ICC value $\geq$ 0.75 was regarded as excellent reliability.

\section{Results}

Four participants were included in the pilot study. The intra-rater reliability for Tdi during inspiration and expiration $(I C C=0.85 ; I C C=0.96$ ) and the inter-rater reliability for the measurements of Tdi during inspiration and expiration $(I C C=0.94 ; \quad I C C=0.90)$ were established. The intra-rater and inter-rater reliability for the measurements of the quadriceps muscle are represented in Table I and Table II.

Table I: Results of intra-rater reliability for ultrasonography of the diaphragm and quadriceps muscle

\begin{tabular}{|c|c|c|c|c|}
\hline \multicolumn{2}{|c|}{ Intra-rater Reliability } \\
\hline & \multicolumn{2}{|c|}{ PI } & \multicolumn{2}{c|}{ Col } \\
\hline & $I C C$ & $P$ & 0.85 & 0.07 \\
\hline Tdi at End - inspiration & 0.94 & 0.03 & 0.96 & 0.02 \\
\hline Tdi at End - expiration & 0.89 & 0.06 & 0.99 & 0.00 \\
\hline RF Muscle Thickness & 0.88 & 0.06 & 0.97 & 0.01 \\
\hline RF Muscle Echogenicity & 0.60 & 0.20 & 0.99 & 0.00 \\
\hline VI Muscle Thickness & 0.97 & 0.01 & 0.84 & 0.08 \\
\hline VI Muscle Echogenicity & 0.75 & 0.13 & \\
\hline
\end{tabular}

Tdi = Diaphragm Thickness; RF = Rectus Femoris; VI = Vastus Intermedius; PI = Primary Investigator; Col $=$ Co-investigator 
Table II: Results of inter-rater reliability for ultrasonography of the diaphragm and quadriceps muscle

\begin{tabular}{|c|c|c|}
\hline \multicolumn{2}{|c|}{ Inter-rater Reliability } \\
\hline & \multicolumn{2}{|c|}{ PI \& Col } \\
\hline & $I C C$ & $P$ \\
\hline Tdi at End - inspiration & 0.94 & 0.00 \\
\hline Tdi at End - expiration & 0.90 & 0.00 \\
\hline RF Muscle Thickness & 0.96 & 0.00 \\
\hline RF Muscle Echogenicity & 0.20 & 0.33 \\
\hline VI Muscle Thickness & 0.95 & 0.00 \\
\hline VI Muscle Echogenicity & 0.94 & 0.00 \\
\hline
\end{tabular}

Tdi = Diaiphragm Thickness; RF = Rectus Femoris; VI = Vastus Intermedius; PI = Primary Investigator; Col $=$ Co-investigator

\section{Discussion}

Excellent intraclass coefficients were yielded for both the $\mathrm{PI}$ and co-investigator intra-rater reliability for Tdi at end-inspiration $(P=0.03 ; P=0.07)$, Tdi at endexpiration $(P=0.06 ; P=0.02)$, RF muscle thickness $(P=0.06 ; P=0.00)$, RF muscle echogenicity $(P=0.20 ; P=0.01)$, VI muscle thickness $(P=0.01 ; P=0.00)$ and $\mathrm{VI}$ muscle echogenicity $(P=0.13 P=0.08)$. Similarly, excellent intraclass coefficients were yielded for inter-rater reliability of Tdi at end-inspiration $(P=0.00)$, Tdi at end-expiration $(P=0.0)$, RF muscle thickness $(P=0.0)$, VI muscle thickness $(P=0.00)$ and $\mathrm{VI}$ muscle echogenicity $(P=0.00)$, except for the measurement of RF muscle echogenicity $(P=33)$. This could be as a result of a measurement error.

\section{Conclusion}

This pilot study proves that udltrasound is a reliable tool and can be used consistently in a clinical setting to measure diaphragm and quadriceps muscle structure. 


\section{Addendum J: REDCap Forms}

\section{Confidential}

\section{Participant info}

\begin{tabular}{l}
\hline Record ID \\
\hline Name \\
\hline Surname number \\
\hline Hospital folder number \\
\hline Contact number \\
\hline Contact number 2 \\
\hline Contact number 3 \\
\hline Address \\
\hline Next of kin name
\end{tabular}

Next of kin contact 


\section{Confidential}

\section{Demographics}

\begin{tabular}{|c|c|}
\hline \multicolumn{2}{|l|}{ Record ID } \\
\hline Study Site & $\begin{array}{l}\bigcirc \text { Tygerberg } \\
\text { Groote Schuur } \\
\bigcirc \text { Vergelegen } \\
\text { Union }\end{array}$ \\
\hline \multicolumn{2}{|l|}{ Unit } \\
\hline \multicolumn{2}{|l|}{ Bed number } \\
\hline Gender & Male $\bigcirc$ Female \\
\hline \multicolumn{2}{|l|}{ Date of Birth (D-M-Y) } \\
\hline \multicolumn{2}{|l|}{ Date of assessment (D-M-Y) } \\
\hline \multicolumn{2}{|l|}{ Age } \\
\hline \multicolumn{2}{|l|}{ Weight (kg) } \\
\hline Employed before admission & $O$ Yes $\bigcirc$ No \\
\hline \multicolumn{2}{|l|}{ Date of admission to TBH } \\
\hline \multicolumn{2}{|l|}{$\begin{array}{l}\text { Which ward was the patient in prior to admission to } \\
\text { ICU? }\end{array}$} \\
\hline \multicolumn{2}{|l|}{ Date and time of admission to ICU } \\
\hline \multicolumn{2}{|l|}{ Time in hospital before ICU admission } \\
\hline Was the patient transferred from another hospital & $O$ Yes $O$ No \\
\hline Name of preceding hospital & \\
\hline
\end{tabular}

Date of admission to preceding hospital (D-M-Y) 


\section{Confidential}

Date of Intubation (D-M-Y)

Time intubated

projectredcap.org REDCap 


\section{Confidential}

\section{Past Med Hx}

\begin{tabular}{|c|c|}
\hline Record ID & \\
\hline Co-morbidities & $\begin{array}{l}\square \text { Hypertension } \\
\square \text { Diabetes } \\
\square \text { COPD } \\
\square \text { Asthma } \\
\square \text { TB } \\
\square \text { Past TB } \\
\square \text { RVD } \\
\square \text { CVA } \\
\square \text { Mental health } \\
\square \text { Other }\end{array}$ \\
\hline \multicolumn{2}{|c|}{ Date of CVA (year) } \\
\hline \multicolumn{2}{|c|}{ Side of hemiplegia } \\
\hline \multicolumn{2}{|l|}{ Please specify } \\
\hline \multicolumn{2}{|c|}{ If other, please specify } \\
\hline \multicolumn{2}{|c|}{ Number of co-morbidities } \\
\hline Smoking history & $\begin{array}{l}\text { Current } \\
\text { Ex-smoker } \\
\text { Never } \\
\bigcirc \text { Unknown }\end{array}$ \\
\hline Illicit drug use & $\bigcirc$ Yes $O$ No $\bigcirc$ Unknown \\
\hline \multicolumn{2}{|c|}{ Name(s) of drugs used (separate with comma) } \\
\hline & $\overline{((\text { separate with comma }))}$ \\
\hline
\end{tabular}




\section{Confidential}

\section{Current Med Hx}

\begin{tabular}{|c|c|}
\hline Record ID & \\
\hline Admission category & $\begin{array}{l}\square \text { Trauma } \\
\square \text { Surgery } \\
\square \text { Medical }\end{array}$ \\
\hline Admission type & $\begin{array}{l}\bigcirc \text { Elective } \\
\text { Emergency }\end{array}$ \\
\hline If Trauma, please specify category & $\begin{array}{l}\square \text { Upper limb fractures } \\
\square \text { Lower limb fractures } \\
\square \text { Spinal fractures } \\
\square \text { Rib fractures } \\
\square \text { Abdominal trauma } \\
\square \text { Head injury } \\
\square \text { Other }\end{array}$ \\
\hline If surgical, please specify & $\begin{array}{l}\square \text { Neurosurgery } \\
\square \text { Abdominal surgery } \\
\square \text { Vascular surgery } \\
\square \text { Plastic surgery } \\
\square \text { Other }\end{array}$ \\
\hline If Medical, please specify a category & $\begin{array}{l}\square \text { Overdose } \\
\square \text { Asthma } \\
\square \text { COPD } \\
\square \text { Cardiac arrest } \\
\square \text { Pneumonia } \\
\square \text { Sepsis } \\
\square \text { Other }\end{array}$ \\
\hline Severity score & $\begin{array}{l}\square \text { APACHE ॥ } \\
\square \text { SOFA } \\
\square \text { SAP ॥ } \\
\square \text { None }\end{array}$ \\
\hline
\end{tabular}

APACHE ॥ Score

(Will pull through from APACHE II instrument)

SOFA Score

(Will pull through from SOFA instrument)

\begin{tabular}{ll}
\hline Bloods taken on admission & Y Yes $\bigcirc$ No \\
\hline Date of bloods (D-M-Y) & \\
\hline CRP (if available) & \\
HGT & REDCap
\end{tabular}




\section{Confidential}

Lactate

Creatinine

Comments 


\section{Confidential}

\section{Daily Assessment}

\begin{tabular}{|c|c|}
\hline Record ID & \\
\hline Date and time of ass & \\
\hline Hours intubated & \\
\hline Mode of ventilation & $\begin{array}{l}\text { BiPap } \\
\text { PC SIMV } \\
\text { VC SIMV } \\
\text { APRV } \\
\text { APAP } \\
\text { Other }\end{array}$ \\
\hline
\end{tabular}

\section{If other}

Pressure support $(\mathrm{cmH2O})$

(Mode over previous 24 hours)

(over preceding 24 hours)

PEEP (cmH2O)
(Mode over previous 24 hours)

(over preceding 24 hours)

Peak pressure
(Mode over previous 24 hours)

(Mode over previous 24 hours) $\quad$ (over preceding 24 hours)

Dynamic compliance $(\mathrm{L} / \mathrm{cmH} 2 \mathrm{O})$

(Mode over previous 24 hours - if available)

(over preceding 24 hours)

Preset RR

(Mode over previous 24 hours)

Recorded RR

(Mode over previous 24 hours)

Has the patient had spontaneous breaths in the preceding 24 hours?

$\bigcirc$ Yes $\bigcirc$ No

Ventilator data for past 24 hours (if available)

Pyrexia in preceding 24 hours $O$ Yes $\bigcirc$ No

Highest recorded temperature in preceding 24 hours

Blood results available (in the last 24 hours)

$\bigcirc$ Yes $\bigcirc$ No 


\section{Confidential}

Date of bloods (D-M-Y)

CRP (if available)

HGT

(morning glucose (nutrition data))

Lactate

Creatinine

White Cell Count

$\mathrm{pH}$
$\mathrm{PaCO} 2$

$\mathrm{PaO} 2$

Is the patient sedated $O$ Yes $\bigcirc$ No

Sedative agent

Dose of sedative

Richmond Agitation and Sedation Scale (RASS)

Overtly combative or violent; immediate danger to staff

3 Pulls on or removes tube(s) or catheter(s) or

has aggressive behavior toward staff

2 Frequent non purposeful movement or

patient-ventilator dyssynchrony

1 Anxious or apprehensive but movements not aggressive or vigorous

0 Spontaneously pays attention to caregiver

$\bigcirc-1$ Not fully alert, but has sustained (more than

10 seconds) awakening, with eye contact, to voice

- -2 Briefly (less than 10 seconds) awakens with

eye contact to voice

-3 Any movement (but no eye contact) to voice

- -4 No response to voice, but any movement to

physical stimulation

-5 No response to voice or physical stimulation

Is the patient receiving corticosteriods?

Yes $O$ No 


\section{Confidential}

If yes, please specify dosage

(over preceding 24 hours)

Is the patient receiving Insulin?

Yes

No

Dosage of insulin

Is the patient CAM-ICU positive

Y Yes

No

$\bigcirc$ Unsure

Comments

Is the patient receiving physiotherapy?

$\bigcirc$ Yes

No

Highest level of rehab achieved

None

Positioning

Passive ROM

AA ROM

Active ROM exercises

Strengthening exercises (resisted)

Bed mobility

Lying to sitting over edge of bed

Sitting to standing

Standing transfer from edge of bed to chair

Marching on the spot

Walking with assistance

Walking independently

Stair climbing 


\section{Confidential}

\section{Ultrasonography}

\begin{tabular}{ll}
\hline Record ID & \\
\hline Instance & $\bigcirc$ Daily \\
& $\bigcirc$ Extubation \\
& $\bigcirc$ ICU discharge \\
& $\bigcirc$ Hospital discharge \\
& $\bigcirc 3$ Months \\
& $\bigcirc 6$ Months \\
& $\bigcirc 12$ Months
\end{tabular}

\begin{tabular}{l}
\hline Diaphragm \\
\hline Date \\
\hline $\begin{array}{l}\text { Diaphragm thickness during inspiration on first } \\
\text { breath (mm) }\end{array}$
\end{tabular}

Diaphragm thickness during inspiration on second breath $(\mathrm{mm})$

Diaphragm thickness during inspiration on third breath $(\mathrm{mm})$

Average diaphragm thickness during inspiration $(\mathrm{mm})$

Diaphragm thickness during expiration on first breath (mm)

Diaphragm thickness during expiration on second breath $(\mathrm{mm})$

Diaphragm thickness during expiration on third breath (mm)

Average diaphragm thickness during expiration $(\mathrm{mm})$

Diaphragm thickening fraction

US measures 


\section{Confidential}

\begin{tabular}{l}
\hline Quads \\
\hline $\mathrm{RF}$ thickness 1 \\
\hline $\mathrm{RF}$ thickness 2 \\
\hline RF thickness 3 \\
\hline RF echogenicity 1 \\
\hline RF echogenicity 2 \\
\hline RF echogenicity 3
\end{tabular}

VI Thickness 1

\section{Thickness 2}

VI Thickness 3

\section{Average VI Thickness}

VI Echogenicity 1

VI Echogenicity 2

VI Echogenicity 3

Average VI Echogenicity 


\section{Confidential}

\section{Muscle Strength}

\begin{tabular}{ll}
\hline Record ID & \\
\hline Instance & O Awakening \\
& Extubation \\
& $\bigcirc$ ICU discharge \\
& $\bigcirc$ Hopsital discharge \\
& $\bigcirc 3$ Months \\
& $\bigcirc$ Months \\
& $\bigcirc 12$ Months \\
\hline
\end{tabular}

Date

\begin{tabular}{ll}
\hline MRC & O Yes $\bigcirc$ No \\
\hline MRC-SS performed & \\
\hline MRC-SS total & \\
\hline Shoulder abduction LEFT & \\
\hline
\end{tabular}

Shoulder abduction RIGHT

Elbow flexion LEFT

Elbow flexion RIGHT

Wrist extension LEFT

Wrist extension RIGHT

Hip flexion LEFT

Hip flexion RIGHT

Knee extension LEFT

Knee extension RIGHT

Ankle dorsiflexion LEFT 


\section{Confidential}

Ankle dorsiflexion RIGHT

\begin{tabular}{ll}
\hline Comments & \\
\hline Dynamometry & \\
\hline Instance & $\bigcirc$ Hopsital discharge \\
& $\bigcirc$ 3 Months \\
& $\bigcirc$ Months \\
& 12 Months \\
\hline Dominant UL & $\bigcirc$ Left $\bigcirc$ Right \\
\hline Dominant LL & $\bigcirc$ Left $\bigcirc$ Right \\
\hline Left knee extension 1 & \\
& \\
\hline
\end{tabular}

Left knee extension 2

Left knee extension 3

\section{Predicted}

$\%$ predicted

Rigth knee extension 1

Rigth knee extension 2

Rigth knee extension 3

\section{Predicted}

$\%$ predicted

Grip strength LEFT 1

Grip strength LEFT 2 


\section{Confidential}

Grip strength LEFT 3

Grip strength LEFT average

Grip strength RIGHT 1

Grip strength RIGHT 2

Grip strength RIGHT 3

Grip strength RIGHT Average 


\section{Confidential}

\section{Respiratory Muscle Strength}

\begin{tabular}{ll}
\hline Record ID & \\
\hline Instance & O Extubation \\
& OICU discharge \\
& O Hopsital discharge \\
& ○ Months \\
& $\bigcirc$ \%onth \\
& \\
& 12 Months
\end{tabular}

Date and time of assessment

First MIP

Second MIP

Third MIP

Best maximal inspiratory pressure

\begin{tabular}{ll}
\hline Endurance \\
\hline $30 \%$ MIP \\
\hline Time completed
\end{tabular}

Number of breaths

Average mean load

Average mean power

Comments 


\section{Confidential}

\section{Physical Function}

\begin{tabular}{ll}
\hline Record ID & \\
\hline Instance & O Awakening \\
& O ICU Discharge \\
& $\bigcirc$ Hospital Discharge \\
& O3 Months \\
& O6 Months \\
& $\bigcirc 12$ Months
\end{tabular}

\section{Date}

S5Q score

(Open and close your eyes; Look at me; Open your mouth and put out your tongue; Nod your head; Raise your eyebrows after I have counted to five)

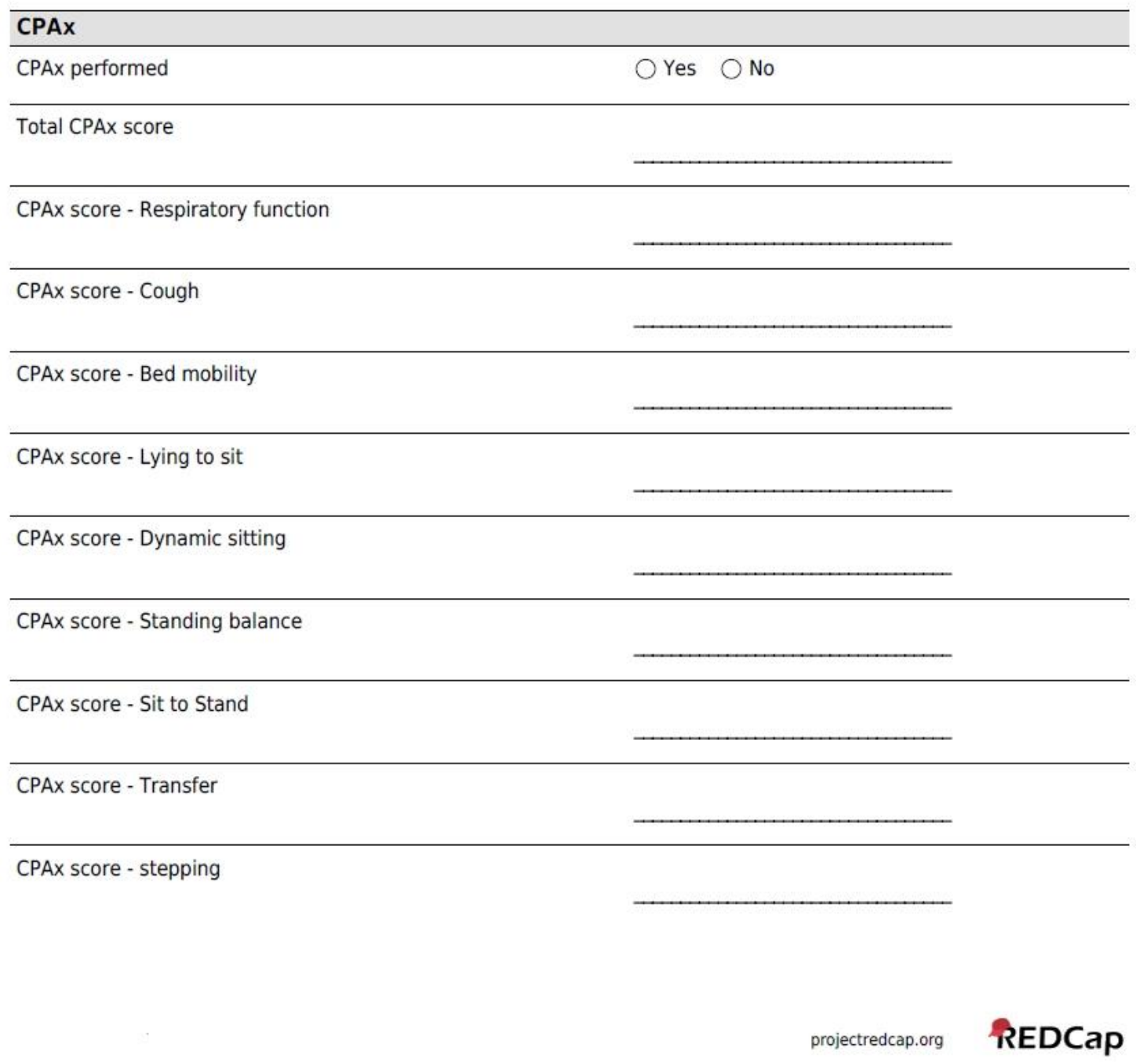




\section{Confidential}

\begin{tabular}{ll}
\hline CPAx score - Grip Strength \\
\hline Hand grip strength 1 \\
\hline Hand grip strength 2 \\
\hline Hand grip strength 3 \\
\hline Best Grip Strength \\
\hline DEMMI \\
\hline Bridge \\
\hline
\end{tabular}

Roll onto side

$(0=$ unable; $1=$ able $)$

Lying to sitting

$\overline{0}=$ unable; $1=\min$ assistance $/$ supervision; $2=$ independent)

Sit unsupported in chair

$(0=$ unable; $1=10 \mathrm{sec})$

Sit to stand from chair

(0 = unable; $1=\min$ assistance $/$ supervision; 2 = independent)

Sit to stand without using arms

$(0=$ unable; $1=$ able $)$

Stand unsupported (no aid)

$(0=$ unable; $1=10 \mathrm{sec})$

Stand feet together

$(0=$ unable; $1=10 \mathrm{sec})$

Stand on toes

$(0=$ unable; $1=10 \mathrm{sec})$ 


\section{Confidential}

Tandem stand with eyes closed

$(0=$ unable; $1=10 \mathrm{sec})$

Walking distance (with or without gait aid)

$\overline{(0=\text { unable } / 5 \mathrm{~m} ; 1=10 \mathrm{~m} / 20 \mathrm{~m} ; 2}=50 \mathrm{~m})$

\begin{tabular}{ll}
\hline Gait aid used & กone \\
& $\bigcirc$ frame \\
$\bigcirc$ stick & $\bigcirc$ other
\end{tabular}

Walking independence

$\bar{c} 0$ unable/min assistance/supervision; 1 = independent with gait aid; 2 = independent without aid)

Pick up pen from floor

$(0=$ unable; $1=$ able $)$

Walks 4 steps backwards

$(0=$ unable; $1=$ able $)$

Jump

(0 = unable; $1=$ able $)$

DEMMI score raw 


\section{Confidential}

\section{Six Minute Walk Test}

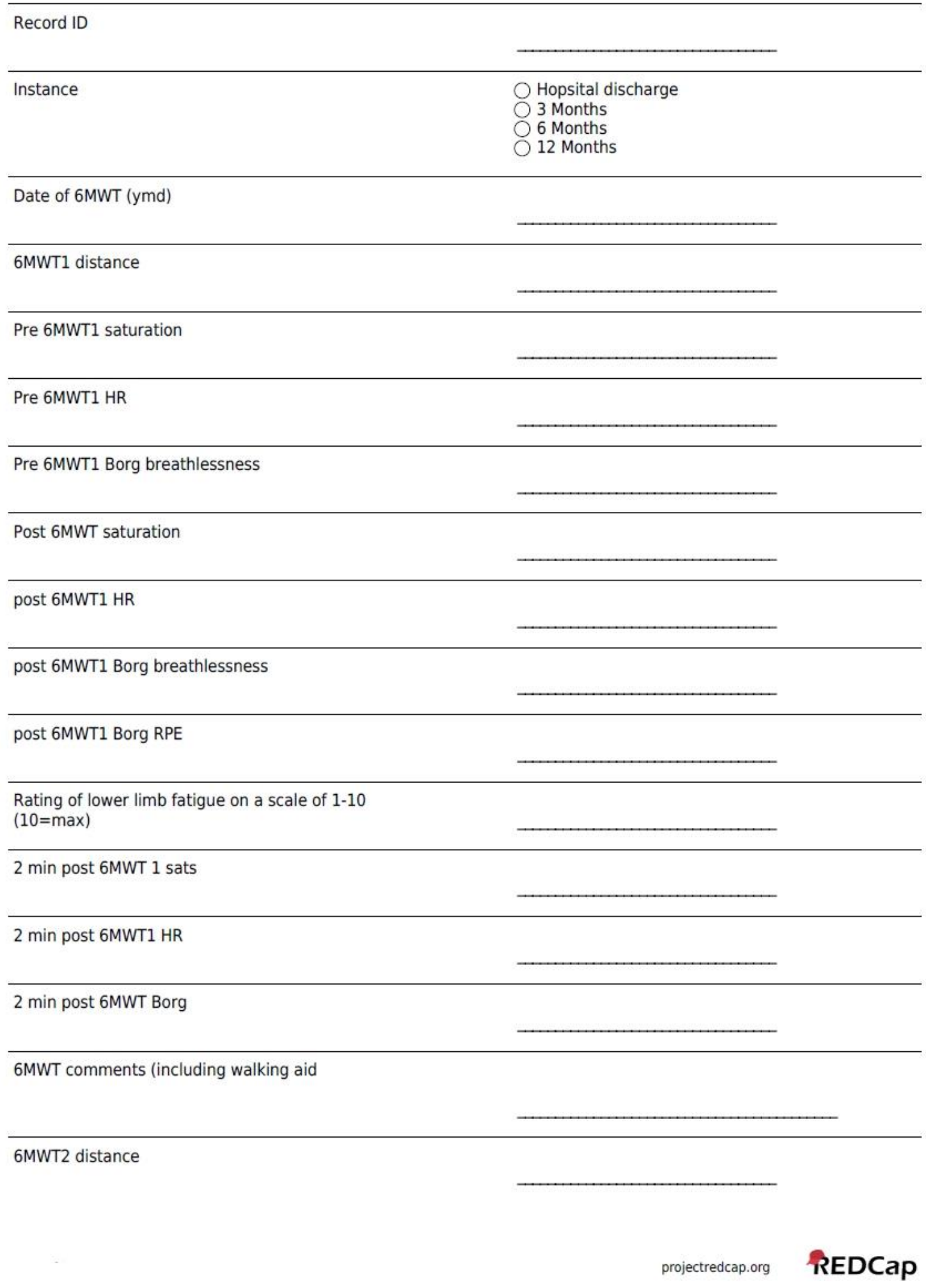




\section{Confidential}

\section{Pre 6MWT2 saturation}

Pre 6MWT2 HR

Pre 6MWT2 Borg breathlessness

Post 6MWT saturation

post $6 M W T 2$ HR

post 6MWT2 Borg breathlessness

post 6MWT2 Borg RPE

Rating of lower limb fatigue on a scale of 1-10

$(10=\max )$

6MWT comments

Best 6MWT distance

Expected 6MWT distance

Percentage of normal 


\section{Confidential}

Quality Of Life Measures

\begin{tabular}{ll}
\hline Record ID & \\
\hline Instance & \\
& $\bigcirc$ Hopsital discharge \\
& $\bigcirc 3$ Months \\
& $\bigcirc 6$ Months \\
& $\bigcirc 12$ Months
\end{tabular}

\section{EQ5D}

Mobility score

Self-care score

Usual Activity score

Pain score

Anxiety/Depression score

VAS Score 


\section{Addendum K: Richmond Agitation and Sedation Scale (RASS)}

Table 1: Number of participants for each level on the RASS

\begin{tabular}{|c|c|c|c|c|}
\hline \multicolumn{5}{|c|}{ Richmond Agitation and Sedation Scale (RASS) } \\
\hline & & \multicolumn{3}{|c|}{ Number of Participants } \\
\hline & & $\begin{array}{l}\text { Day } 1 \\
(n=45)\end{array}$ & $\begin{array}{l}\text { Day } 2 \\
(\mathrm{n}=31)\end{array}$ & $\begin{array}{l}\text { Day } 3 \\
(n=29)\end{array}$ \\
\hline-5 & No response to voice or physical stimulation & 9 & 2 & 1 \\
\hline-4 & $\begin{array}{l}\text { No response to voice, but any movement to } \\
\text { physical stimulation }\end{array}$ & 3 & 2 & 2 \\
\hline-3 & Any movement (but no eye contact) to voice & 6 & 5 & 5 \\
\hline-2 & $\begin{array}{l}\text { Briefly (less than } 10 \text { seconds) awakens with } \\
\text { eye contact to voice }\end{array}$ & 9 & 3 & 1 \\
\hline-1 & $\begin{array}{l}\text { Not fully alert, but has sustained (more than } \\
10 \text { seconds) awakening, with eye contact, to } \\
\text { voice }\end{array}$ & 10 & 4 & 2 \\
\hline 0 & Spontaneously pays attention to caregiver & 5 & 13 & 14 \\
\hline 1 & $\begin{array}{c}\text { Anxious or apprehensive but movements not } \\
\text { aggressive or vigorous }\end{array}$ & 3 & 1 & 4 \\
\hline 2 & $\begin{array}{l}\text { Frequent non purposeful movement or patient- } \\
\text { ventilator dyssynchrony }\end{array}$ & 0 & 1 & 0 \\
\hline 3 & $\begin{array}{l}\text { Pulls on or removes tube(s) or catheter(s) or } \\
\text { has aggressive behaviour toward staff }\end{array}$ & 0 & 0 & 0 \\
\hline
\end{tabular}

